Supplementary Information

\title{
Identification of small molecules of the infective stage of human hookworm using LCMS-based metabolomics and lipidomics protocols
}

Phurpa Wangchuk ${ }^{1 *}$, Dovile Anderson ${ }^{2}$, Karma Yeshi ${ }^{1}$, Alex Loukas ${ }^{1}$

${ }^{1}$ Centre for Molecular Therapeutics, Australian Institute of Tropical Health and Medicine, James Cook University, Building E4, McGregor Rd, Smithfield, Cairns, QLD 4878, Australia

2 Monash Institute of Pharmaceutical Sciences, Monash University, Royal Parade, Parkville, Victoria 3052, Australia.

* Corresponding author: Phurpa Wangchuk (phurpa.wangchuk@jcu.edu.au) 
A) $\mathrm{C}_{31} \mathrm{H}_{63} \mathrm{O}_{3} \quad \mathrm{~m} / \mathrm{z} 483.4779$

OH

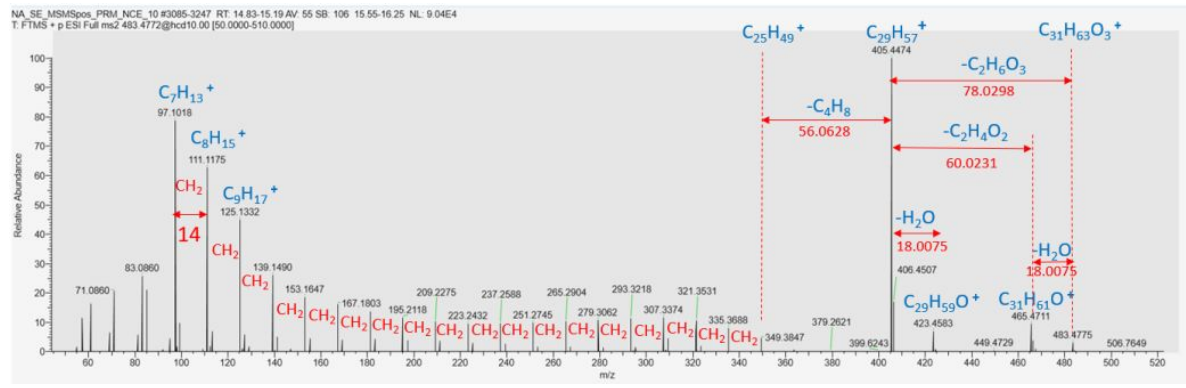

B) $\mathrm{C}_{33} \mathrm{H}_{66} \mathrm{O}_{3} \quad \mathrm{~m} / \mathrm{z} 511.5094$
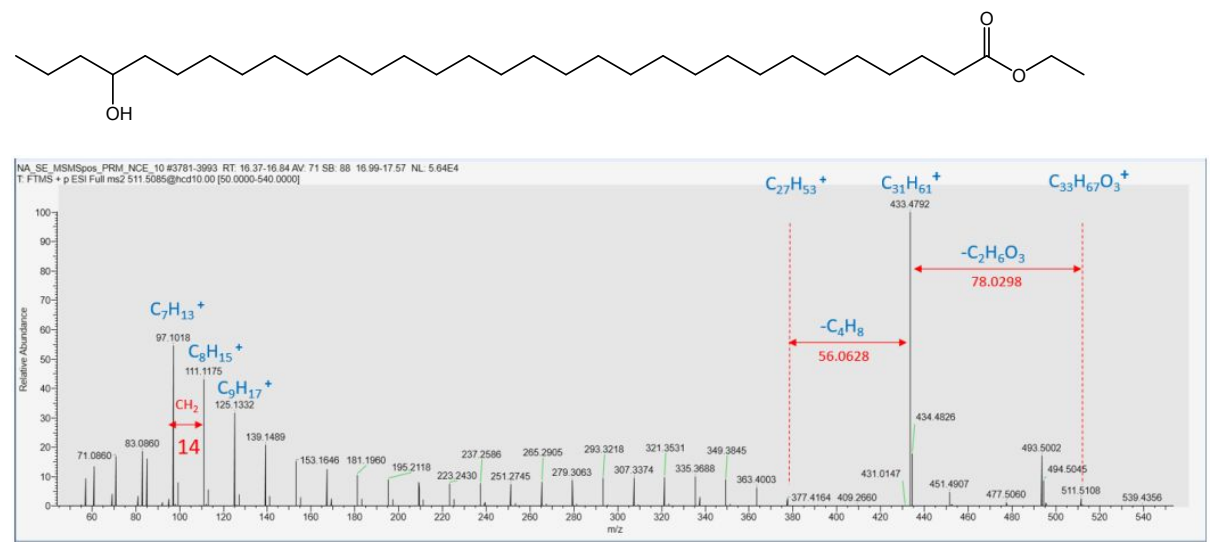

C) $\mathrm{C}_{37} \mathrm{H}_{74} \mathrm{O}_{3} \quad \mathrm{~m} / \mathrm{z} 567.5725$
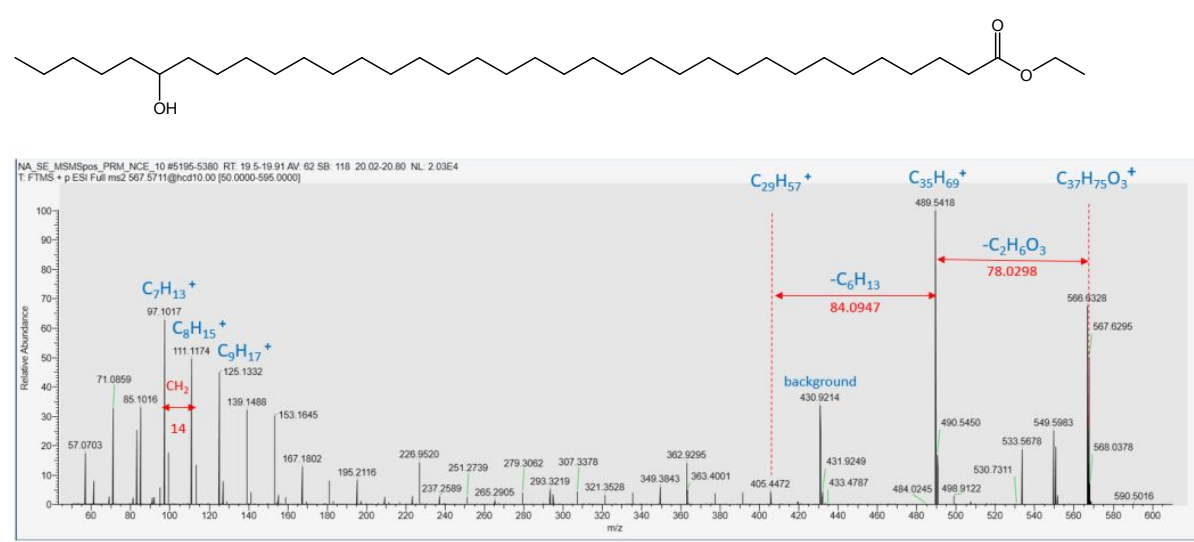

Figure S1. Proposed MS2 spectra assignment of novel fatty acid derivatives (A-C). MS2 spectra were recorded on QExactive mass spectrometer (Thermo Fisher Scientific) using chromatography conditions described in the Methods section. PRM scan mode in positive ion mode with collision energy NCE 10 was used. Accurate mass MS2 spectra presented here were obtained by averaging chromatographic region and subtracting background from both sides of the peak. Molecular formulas corresponding to product ions and neutral losses are shown. Long chains $\mathrm{C}_{25}, \mathrm{C}_{27}$ and $\mathrm{C}_{29}$ are observed for homologous fatty acid derivatives and corresponds to the part of the chain between carboxylic groups and hydroxyl group. The terminal part of the chain located after hydroxyl group is observed as neutral loss $-\mathrm{C}_{4}$ and $\mathrm{C}_{6}$ chains. Ester group is identified by neutral loss of $\mathrm{C}_{2} \mathrm{H}_{6} \mathrm{O}_{3}$ which leads to formation of the highest intensity product ions $-\mathrm{C}_{29} \mathrm{H}_{57}{ }^{+}, \mathrm{C}_{31} \mathrm{H}_{61}{ }^{+}$and $\mathrm{C}_{35} \mathrm{H}_{69}{ }^{+}$. 
Table S1: Total putative polar metabolites identified in the somatic extract (SE) and excretory/secretory products (ESP) of the infective third stage larva (L3) of Necator americanus.

\begin{tabular}{|c|c|c|c|c|c|c|c|c|c|c|c|c|c|c|c|c|}
\hline \multirow[t]{2}{*}{$\begin{array}{l}\text { Putative } \\
\text { Metabolites }\end{array}$} & \multirow[t]{2}{*}{$\begin{array}{l}\text { Mass } \\
(m / z)\end{array}$} & \multirow[t]{2}{*}{$\begin{array}{l}\text { Retention } \\
\text { time (min) }\end{array}$} & \multirow[t]{2}{*}{$\begin{array}{l}\text { Molecular } \\
\text { formula }\end{array}$} & \multirow[t]{2}{*}{$\begin{array}{l}\text { Metabolic } \\
\text { pathways (MAP) }\end{array}$} & \multicolumn{6}{|c|}{ Peak height values $(\mathrm{mz} / \mathrm{rt}$ ) for somatic extract (NA_SE) replicates } & \multicolumn{6}{|c|}{$\begin{array}{l}\text { Peak height values (mz/rt) for excretory/secretory products (NA_ESP) } \\
\text { replicates }\end{array}$} \\
\hline & & & & & NA_SE_1 & NA_SE_2 & NA_SE_3 & NA_SE_4 & NA_SE_5 & NA_SE_6 & $\begin{array}{l}\text { NA_ESP } \\
-1\end{array}$ & ${ }_{2}^{\text {NA_ESP_ }}$ & $\begin{array}{l}\text { NA_ESP } \\
-3\end{array}$ & $\begin{array}{l}\text { NA_ESP } \\
-4\end{array}$ & $\begin{array}{l}\text { NA_ESP } \\
-5\end{array}$ & NA_ESP_6 \\
\hline Ile-lle-Phe & 391.2 & 3.6 & $\begin{array}{l}\mathrm{C} 21 \mathrm{H} 33 \mathrm{~N} 3 \\
\mathrm{O} 4\end{array}$ & Peptide(tri-) & 260101.9 & 289403.7 & 389503.5 & 514961.4 & $\begin{array}{l}1156902 . \\
6\end{array}$ & 566372.4 & 0.0 & 0.0 & 0.0 & 0.0 & 0.0 & 0.0 \\
\hline Gln-Leu-Lys-Tyr & 550.3 & 27.2 & $\begin{array}{l}\mathrm{C} 26 \mathrm{H} 42 \mathrm{~N} 6 \\
\mathrm{O} 7\end{array}$ & Peptide(tetra-) & 247423.2 & 261023.0 & 270790.6 & 253167.6 & 264979.5 & 253349.7 & 0.0 & 0.0 & 0.0 & 0.0 & 0.0 & 0.0 \\
\hline Ile-Val & 230.2 & 4.7 & $\begin{array}{l}\mathrm{C} 11 \mathrm{H} 22 \mathrm{~N} 2 \\
\mathrm{O} 3\end{array}$ & Peptide(di-) & 237705.0 & 520075.0 & 839975.4 & 461793.4 & 400439.0 & 624871.1 & 0.0 & 0.0 & 0.0 & 0.0 & 0.0 & 0.0 \\
\hline Glycyl-leucine & 188.1 & 11.9 & $\begin{array}{l}\mathrm{C} 8 \mathrm{H} 16 \mathrm{~N} 2 \mathrm{O} \\
3\end{array}$ & Peptide(di-) & 136882.3 & 178243.2 & 111644.5 & 201234.2 & 79304.4 & $\begin{array}{l}1102088 . \\
5\end{array}$ & 0.0 & 0.0 & 0.0 & 0.0 & 0.0 & 0.0 \\
\hline AMP & 347.1 & 13.0 & $\begin{array}{l}\mathrm{C} 10 \mathrm{H} 14 \mathrm{~N} 5 \\
\text { O7P }\end{array}$ & $\begin{array}{l}\text { Nucleotide } \\
\text { Metabolism }\end{array}$ & $\begin{array}{l}1619727 . \\
5\end{array}$ & $\begin{array}{l}3836109 . \\
0\end{array}$ & $\begin{array}{l}1854237 . \\
6\end{array}$ & $\begin{array}{l}2549816 . \\
8\end{array}$ & 925008.4 & $\begin{array}{l}1482267 \\
7.0\end{array}$ & 0.0 & 0.0 & 0.0 & 0.0 & 0.0 & 0.0 \\
\hline Sulfate & 98.0 & 18.3 & $\mathrm{H} 2 \mathrm{O} 4 \mathrm{~S}$ & $\begin{array}{l}\text { Nucleotide } \\
\text { Metabolism }\end{array}$ & 712392.6 & 361416.3 & 352675.5 & 619517.5 & 371630.1 & $\begin{array}{l}1124599 . \\
3\end{array}$ & 0.0 & 0.0 & 0.0 & 0.0 & 0.0 & 0.0 \\
\hline Adenosine & 267.1 & 8.2 & $\begin{array}{l}\mathrm{C} 10 \mathrm{H} 13 \mathrm{~N} 5 \\
\mathrm{O} 4\end{array}$ & $\begin{array}{l}\text { Nucleotide } \\
\text { Metabolism }\end{array}$ & 255729.2 & 324320.3 & 453652.0 & 330963.1 & 137543.3 & 647226.9 & 0.0 & 0.0 & 0.0 & 0.0 & 0.0 & 0.0 \\
\hline Inosine & 268.1 & 10.3 & $\begin{array}{l}\mathrm{C} 10 \mathrm{H} 12 \mathrm{~N} 4 \\
\mathrm{O} 5\end{array}$ & $\begin{array}{l}\text { Nucleotide } \\
\text { Metabolism }\end{array}$ & 235399.9 & 231529.5 & 322743.2 & 279925.1 & 103032.5 & 333640.6 & 0.0 & 0.0 & 0.0 & 0.0 & 0.0 & 0.0 \\
\hline Uracil & 112.0 & 9.3 & $\mathrm{C} 4 \mathrm{H} 4 \mathrm{~N} 2 \mathrm{O} 2$ & $\begin{array}{l}\text { Nucleotide } \\
\text { Metabolism }\end{array}$ & 48393.5 & 50613.4 & 63319.2 & 64215.1 & 34257.7 & 70320.5 & 0.0 & 0.0 & 0.0 & 0.0 & 0.0 & 0.0 \\
\hline Hypoxanthine & 136.0 & 9.6 & $\mathrm{C} 5 \mathrm{H} 4 \mathrm{~N} 4 \mathrm{O}$ & $\begin{array}{l}\text { Nucleotide } \\
\text { Metabolism }\end{array}$ & $\begin{array}{l}1728479 . \\
9\end{array}$ & $\begin{array}{l}2264428 . \\
0\end{array}$ & $\begin{array}{l}2583634 . \\
3\end{array}$ & $\begin{array}{l}3600937 . \\
8\end{array}$ & $\begin{array}{l}1658013 . \\
4\end{array}$ & $\begin{array}{l}4109222 . \\
5\end{array}$ & $\begin{array}{l}218075 . \\
8\end{array}$ & 329078.0 & 71416.5 & 0.0 & 46792.9 & 37372.7 \\
\hline $\begin{array}{l}\text { 3- } \\
\text { Ureidoisobutyrate }\end{array}$ & 146.1 & 7.3 & $\begin{array}{l}\text { C5H10N2O } \\
3\end{array}$ & $\begin{array}{l}\text { Nucleotide } \\
\text { Metabolism }\end{array}$ & 196944.5 & 346036.3 & 173237.5 & 87737.1 & 17610.1 & $\begin{array}{l}1519119 . \\
6\end{array}$ & $\begin{array}{l}917270 . \\
3\end{array}$ & $\begin{array}{l}1309394 \\
8.0\end{array}$ & $\begin{array}{l}263222 \\
8.5\end{array}$ & $\begin{array}{l}169499 \\
6.8\end{array}$ & $\begin{array}{l}462608 \\
5.5\end{array}$ & 2930031.3 \\
\hline $\begin{array}{l}5,6- \\
\text { Dihydrothymine }\end{array}$ & 128.1 & 8.6 & $\mathrm{C} 5 \mathrm{H} 8 \mathrm{~N} 2 \mathrm{O} 2$ & $\begin{array}{l}\text { Nucleotide } \\
\text { Metabolism }\end{array}$ & 71066.1 & 44001.4 & 44479.5 & 50781.1 & 9422.8 & 246443.4 & 0.0 & 0.0 & 0.0 & 0.0 & 0.0 & 0.0 \\
\hline cAMP & 329.1 & 8.7 & $\begin{array}{l}\mathrm{C} 10 \mathrm{H} 12 \mathrm{~N} 5 \\
\text { O6P }\end{array}$ & $\begin{array}{l}\text { Nucleotide } \\
\text { Metabolism }\end{array}$ & 0.0 & 0.0 & 0.0 & 0.0 & 0.0 & 20632.1 & 0.0 & 0.0 & 0.0 & 0.0 & 0.0 & 0.0 \\
\hline
\end{tabular}




\begin{tabular}{|c|c|c|c|c|c|c|c|c|c|c|c|c|c|c|c|c|}
\hline Xanthine & 152.0 & 11.1 & $\mathrm{C} 5 \mathrm{H} 4 \mathrm{~N} 4 \mathrm{O} 2$ & $\begin{array}{l}\text { Nucleotide } \\
\text { Metabolism }\end{array}$ & $\begin{array}{l}1406840 . \\
5\end{array}$ & $\begin{array}{l}2734506 . \\
0\end{array}$ & $\begin{array}{l}1629396 . \\
4\end{array}$ & $\begin{array}{l}2414087 . \\
3\end{array}$ & 560187.1 & $\begin{array}{l}7040238 . \\
0\end{array}$ & $\begin{array}{l}286313 . \\
8\end{array}$ & 213305.5 & $\begin{array}{l}248172 . \\
8\end{array}$ & 0.0 & $\begin{array}{l}184273 . \\
6\end{array}$ & 0.0 \\
\hline Adenine & 135.1 & 8.3 & C5H5N5 & $\begin{array}{l}\text { Nucleotide } \\
\text { Metabolism }\end{array}$ & 55187.8 & 71102.2 & 98255.9 & 74491.1 & 38231.4 & 122283.3 & 94745.7 & 620813.6 & $\begin{array}{l}252713 . \\
4\end{array}$ & 46229.4 & $\begin{array}{l}132488 . \\
0\end{array}$ & 122700.3 \\
\hline 7-Methyladenine & 149.1 & 10.9 & $\mathrm{C} 6 \mathrm{H} 7 \mathrm{~N} 5$ & $\begin{array}{l}\text { Nucleotide } \\
\text { Metabolism }\end{array}$ & 31794.6 & 72604.0 & 42640.9 & 59611.4 & 16888.4 & 200270.3 & 0.0 & 0.0 & 0.0 & 0.0 & 0.0 & 0.0 \\
\hline 7-oxoheptanoate & 144.1 & 4.8 & $\mathrm{C} 7 \mathrm{H} 12 \mathrm{O} 3$ & $\begin{array}{l}\text { Metabolism of } \\
\text { Cofactors and } \\
\text { Vitamins }\end{array}$ & 418252.3 & 373072.6 & 318569.2 & 316181.2 & 123294.2 & 253475.8 & 0.0 & 0.0 & 0.0 & 0.0 & 0.0 & 0.0 \\
\hline $\begin{array}{l}\text { 2,3- } \\
\text { Dimethylmaleate }\end{array}$ & 144.0 & 13.0 & $\mathrm{C} 6 \mathrm{H} 8 \mathrm{O} 4$ & $\begin{array}{l}\text { Metabolism of } \\
\text { Cofactors and } \\
\text { Vitamins }\end{array}$ & 166055.4 & 26543.2 & 114470.8 & 139347.1 & 55462.3 & 150162.5 & 0.0 & 0.0 & 0.0 & 0.0 & 0.0 & 0.0 \\
\hline 4-Hydroxybenzoate & 138.0 & 10.4 & $\mathrm{C} 7 \mathrm{H} 6 \mathrm{O} 3$ & $\begin{array}{l}\text { Metabolism of } \\
\text { Cofactors and } \\
\text { Vitamins }\end{array}$ & 20860.6 & 33460.6 & 18154.8 & 30580.0 & 18389.2 & 52911.1 & 0.0 & 0.0 & 0.0 & 0.0 & 0.0 & 0.0 \\
\hline Pyridoxal & 167.1 & 7.5 & $\mathrm{C} 8 \mathrm{H} 9 \mathrm{NO} 3$ & $\begin{array}{l}\text { Metabolism of } \\
\text { Cofactors and } \\
\text { Vitamins }\end{array}$ & 11843.7 & 15334.6 & 11965.7 & 12799.8 & 0.0 & 10789.9 & 0.0 & 0.0 & 0.0 & 0.0 & 0.0 & 0.0 \\
\hline $\begin{array}{l}\text { N- } \\
\text { Ribosylnicotinamid } \\
\text { e }\end{array}$ & 254.1 & 24.1 & $\begin{array}{l}\text { C11H14N2 } \\
05\end{array}$ & $\begin{array}{l}\text { Metabolism of } \\
\text { Cofactors and } \\
\text { Vitamins }\end{array}$ & 9168.7 & 10863.3 & 14986.4 & 5015.0 & 3694.2 & 11599.2 & 0.0 & 0.0 & 0.0 & 0.0 & 0.0 & 0.0 \\
\hline $\begin{array}{l}\text { 8-Amino-7- } \\
\text { oxononanoate }\end{array}$ & 187.1 & 4.7 & $\mathrm{C} 9 \mathrm{H} 17 \mathrm{NO} 3$ & $\begin{array}{l}\text { Metabolism of } \\
\text { Cofactors and } \\
\text { Vitamins }\end{array}$ & 85900.9 & 90058.1 & 87770.6 & 132299.7 & 48579.2 & 50760.6 & 0.0 & 0.0 & 0.0 & 0.0 & 0.0 & 0.0 \\
\hline Orthophosphate & 98.0 & 15.7 & $\mathrm{H} 304 \mathrm{P}$ & $\begin{array}{l}\text { Energy } \\
\text { Metabolism }\end{array}$ & $\begin{array}{l}1370573 \\
5.0\end{array}$ & $\begin{array}{l}1028799 \\
2.0\end{array}$ & $\begin{array}{l}1958219 \\
8.0\end{array}$ & $\begin{array}{l}1972767 \\
0.0\end{array}$ & $\begin{array}{l}3308786 \\
0.0\end{array}$ & $\begin{array}{l}2313976 \\
4.0\end{array}$ & 0.0 & 0.0 & 0.0 & 0.0 & 0.0 & 0.0 \\
\hline $\begin{array}{l}\text { L-Rhamnono-1,4- } \\
\text { lactone }\end{array}$ & 162.1 & 6.9 & $\mathrm{C} 6 \mathrm{H} 1005$ & $\begin{array}{l}\text { Carbohydrate } \\
\text { Metabolism }\end{array}$ & 49156.5 & 52490.3 & 64188.6 & 41845.6 & 20032.3 & 34619.6 & 0.0 & 0.0 & 0.0 & 0.0 & 0.0 & 0.0 \\
\hline Isocitrate & 192.0 & 19.0 & $\mathrm{C} 6 \mathrm{H} 8 \mathrm{O} 7$ & $\begin{array}{l}\text { Carbohydrate } \\
\text { Metabolism }\end{array}$ & 120949.8 & 102393.0 & 52742.9 & 170626.2 & 35458.3 & $\begin{array}{l}1731855 . \\
5\end{array}$ & $\begin{array}{l}163649 \\
4.6\end{array}$ & $\begin{array}{l}2229708 . \\
5\end{array}$ & $\begin{array}{l}217095 \\
1.5\end{array}$ & $\begin{array}{l}290399 \\
8.0\end{array}$ & $\begin{array}{l}262429 \\
3.5\end{array}$ & 2066580.3 \\
\hline 3-Ethylmalate & 162.1 & 14.1 & $\mathrm{C} 6 \mathrm{H} 1005$ & $\begin{array}{l}\text { Carbohydrate } \\
\text { Metabolism }\end{array}$ & 56699.0 & 52287.8 & 61976.7 & 76757.0 & 61686.0 & 325943.3 & 0.0 & 0.0 & 0.0 & 0.0 & 0.0 & 0.0 \\
\hline D-Galactose & 180.1 & 11.5 & $\mathrm{C} 6 \mathrm{H} 12 \mathrm{O} 6$ & $\begin{array}{l}\text { Carbohydrate } \\
\text { Metabolism }\end{array}$ & 58483.4 & 74216.0 & 45370.6 & 80747.3 & 10944.0 & 453502.0 & 0.0 & 0.0 & 0.0 & 0.0 & 0.0 & 0.0 \\
\hline D-Arabinonate & 166.0 & 13.1 & C5H10O6 & $\begin{array}{l}\text { Carbohydrate } \\
\text { Metabolism }\end{array}$ & 71174.3 & 78398.8 & 70901.4 & 75511.7 & 48708.1 & 264315.2 & 0.0 & 0.0 & 0.0 & 0.0 & 0.0 & 0.0 \\
\hline
\end{tabular}




\begin{tabular}{|c|c|c|c|c|c|c|c|c|c|c|c|c|c|c|c|c|}
\hline $\begin{array}{l}\text { [FA trihydroxy(4:0)] } \\
\text { 2,3,4-trihydroxy- } \\
\text { butanoic acid }\end{array}$ & 136.0 & 12.4 & $\mathrm{C} 4 \mathrm{H} 8 \mathrm{O} 5$ & $\begin{array}{l}\text { Carbohydrate } \\
\text { Metabolism }\end{array}$ & 233944.4 & 281328.0 & 173719.8 & 300805.8 & 80091.4 & $\begin{array}{l}2532494 . \\
0\end{array}$ & 0.0 & 0.0 & 0.0 & 0.0 & 0.0 & 0.0 \\
\hline Mannitol & 182.1 & 13.3 & $\mathrm{C} 6 \mathrm{H} 14 \mathrm{O} 6$ & $\begin{array}{l}\text { Carbohydrate } \\
\text { Metabolism }\end{array}$ & 40142.6 & 25467.6 & 0.0 & 66769.7 & 35086.2 & 46622.8 & 0.0 & 0.0 & 0.0 & 0.0 & 0.0 & 0.0 \\
\hline $\begin{array}{l}2,5- \\
\text { Dioxopentanoate }\end{array}$ & 130.0 & 12.0 & $\mathrm{C} 5 \mathrm{H} 6 \mathrm{O} 4$ & $\begin{array}{l}\text { Carbohydrate } \\
\text { Metabolism }\end{array}$ & 139354.4 & 65010.9 & 98607.7 & 107651.7 & 30625.3 & 181286.1 & 0.0 & 0.0 & 0.0 & 0.0 & 0.0 & 0.0 \\
\hline $\begin{array}{l}\text { 2-Dehydro-3- } \\
\text { deoxy-L- } \\
\text { rhamnonate }\end{array}$ & 162.1 & 15.6 & $\mathrm{C} 6 \mathrm{H} 10 \mathrm{O} 5$ & $\begin{array}{l}\text { Carbohydrate } \\
\text { Metabolism }\end{array}$ & 60127.2 & 88242.6 & 103443.3 & 135962.0 & 71402.9 & 164005.8 & 0.0 & 0.0 & 0.0 & 0.0 & 0.0 & 0.0 \\
\hline (R)-Lactate & 90.0 & 15.5 & С3H6O3 & $\begin{array}{l}\text { Carbohydrate } \\
\text { Metabolism }\end{array}$ & 38937.8 & 52919.0 & 37105.3 & 48396.6 & 25600.2 & 202968.5 & 0.0 & 0.0 & 0.0 & 0.0 & 0.0 & 0.0 \\
\hline Glycerol & 92.0 & 9.9 & $\mathrm{C} 3 \mathrm{H} 8 \mathrm{O} 3$ & $\begin{array}{l}\text { Carbohydrate } \\
\text { Metabolism }\end{array}$ & 27631.1 & 116630.5 & 18093.3 & 51729.6 & 86269.5 & 25452.2 & 0.0 & 0.0 & 0.0 & 0.0 & 0.0 & 0.0 \\
\hline (S)-Malate & 134.0 & 16.2 & $\mathrm{C} 4 \mathrm{H} 6 \mathrm{O} 5$ & $\begin{array}{l}\text { Carbohydrate } \\
\text { Metabolism }\end{array}$ & $\begin{array}{l}1297183 . \\
9\end{array}$ & $\begin{array}{l}1826803 . \\
9\end{array}$ & $\begin{array}{l}2817664 . \\
3\end{array}$ & $\begin{array}{l}6934166 . \\
5\end{array}$ & $\begin{array}{l}2179204 . \\
8\end{array}$ & $\begin{array}{l}6866927 . \\
0\end{array}$ & 0.0 & 0.0 & 0.0 & 0.0 & 0.0 & 0.0 \\
\hline L-Rhamnose & 164.1 & 10.2 & $\mathrm{C} 6 \mathrm{H} 12 \mathrm{O} 5$ & $\begin{array}{l}\text { Carbohydrate } \\
\text { Metabolism }\end{array}$ & 21142.5 & 32288.2 & 13731.3 & 65006.0 & 18905.4 & 44732.3 & 0.0 & 0.0 & 0.0 & 0.0 & 0.0 & 0.0 \\
\hline Lactose & 342.1 & 15.3 & $\mathrm{C} 12 \mathrm{H} 22 \mathrm{O} 11$ & $\begin{array}{l}\text { Carbohydrate } \\
\text { Metabolism }\end{array}$ & $\begin{array}{l}5540634 . \\
0\end{array}$ & $\begin{array}{l}1132332 \\
1.0\end{array}$ & $\begin{array}{l}5265605 . \\
0\end{array}$ & $\begin{array}{l}7360469 . \\
0\end{array}$ & $\begin{array}{l}2502043 . \\
8\end{array}$ & $\begin{array}{l}5430932 \\
8.0\end{array}$ & 0.0 & 0.0 & 0.0 & 0.0 & 0.0 & 0.0 \\
\hline Succinate & 118.0 & 15.2 & $\mathrm{C} 4 \mathrm{H} 6 \mathrm{O} 4$ & $\begin{array}{l}\text { Carbohydrate } \\
\text { Metabolism }\end{array}$ & 492066.7 & 963587.6 & $\begin{array}{l}1143688 . \\
9\end{array}$ & $\begin{array}{l}1191733 . \\
3\end{array}$ & 470339.4 & $\begin{array}{l}4371399 . \\
0\end{array}$ & $\begin{array}{l}179113 . \\
6\end{array}$ & 95119.7 & $\begin{array}{l}253902 . \\
6\end{array}$ & $\begin{array}{l}392402 . \\
3\end{array}$ & $\begin{array}{l}134737 \\
2.0\end{array}$ & 640366.5 \\
\hline (R)-2-Ethylmalate & 162.1 & 15.2 & $\mathrm{C} 6 \mathrm{H} 1005$ & $\begin{array}{l}\text { Carbohydrate } \\
\text { Metabolism }\end{array}$ & 78196.3 & 128581.7 & 129653.7 & 180322.0 & 107024.4 & 254065.9 & 0.0 & 0.0 & 0.0 & 0.0 & 0.0 & 0.0 \\
\hline D-Erythrose & 120.0 & 15.3 & $\mathrm{C} 4 \mathrm{H} 8 \mathrm{O} 4$ & $\begin{array}{l}\text { Carbohydrate } \\
\text { Metabolism }\end{array}$ & 39581.1 & 73142.4 & 32487.3 & 43622.9 & 38761.1 & 217221.3 & 0.0 & 0.0 & 0.0 & 0.0 & 0.0 & 0.0 \\
\hline D-Glycerate & 106.0 & 11.9 & $\mathrm{C} 3 \mathrm{H} 6 \mathrm{O} 4$ & $\begin{array}{l}\text { Carbohydrate } \\
\text { Metabolism }\end{array}$ & 20054.5 & 21063.4 & 21668.3 & 31788.8 & 11124.1 & 52229.5 & 0.0 & 0.0 & 0.0 & 0.0 & 0.0 & 0.0 \\
\hline Hexose phosphate & 260.0 & 16.6 & C6H1309P & $\begin{array}{l}\text { Carbohydrate } \\
\text { Metabolism }\end{array}$ & 341833.0 & 620727.9 & 128361.8 & 230286.3 & 75769.6 & $\begin{array}{l}2136688 . \\
0\end{array}$ & 0.0 & 0.0 & 0.0 & 0.0 & 0.0 & 0.0 \\
\hline D-Arabitol & 152.1 & 12.4 & $\mathrm{C} 5 \mathrm{H} 12 \mathrm{O} 5$ & $\begin{array}{l}\text { Carbohydrate } \\
\text { Metabolism }\end{array}$ & 13298.1 & 16098.9 & 0.0 & 32163.1 & 0.0 & 93150.9 & 0.0 & 0.0 & 0.0 & 0.0 & 0.0 & 0.0 \\
\hline $\begin{array}{l}\text { D-Glucose 6- } \\
\text { phosphate }\end{array}$ & 260.0 & 15.8 & C6H1309P & $\begin{array}{l}\text { Carbohydrate } \\
\text { Metabolism }\end{array}$ & 258852.6 & 439669.2 & 188862.2 & 257855.6 & 62580.0 & $\begin{array}{l}1418203 . \\
4\end{array}$ & 0.0 & 0.0 & 0.0 & 0.0 & 0.0 & 0.0 \\
\hline
\end{tabular}




\begin{tabular}{|c|c|c|c|c|c|c|c|c|c|c|c|c|c|c|c|c|}
\hline 2-Oxoglutarate & 146.0 & 15.8 & $\mathrm{C} 5 \mathrm{H} 6 \mathrm{O} 5$ & $\begin{array}{l}\text { Carbohydrate } \\
\text { Metabolism }\end{array}$ & 72247.2 & 71446.4 & 84276.0 & 247125.6 & 67406.9 & 340239.6 & $\begin{array}{l}220503 . \\
5\end{array}$ & 69041.1 & $\begin{array}{l}443387 . \\
9\end{array}$ & $\begin{array}{l}286299 . \\
7\end{array}$ & $\begin{array}{l}581233 . \\
1\end{array}$ & 461406.5 \\
\hline $\begin{array}{l}\text { 1-O-Methyl-myo- } \\
\text { inositol }\end{array}$ & 194.1 & 10.1 & C7H14O6 & $\begin{array}{l}\text { Carbohydrate } \\
\text { Metabolism }\end{array}$ & 28196.8 & 42850.4 & 33535.6 & 34372.5 & 36861.8 & 53375.4 & 0.0 & 0.0 & 0.0 & 0.0 & 0.0 & 0.0 \\
\hline $\begin{array}{l}\text { 6-endo- } \\
\text { Hydroxycineole }\end{array}$ & 170.1 & 3.8 & $\mathrm{C} 10 \mathrm{H} 18 \mathrm{O} 2$ & $\begin{array}{l}\text { Biosynthesis of } \\
\text { Secondary } \\
\text { Metabolites }\end{array}$ & 170400.1 & 268458.0 & 131405.5 & 122853.0 & 186530.5 & 218644.4 & 0.0 & 0.0 & 0.0 & 0.0 & 0.0 & 0.0 \\
\hline Slaframine & 240.1 & 8.9 & $\begin{array}{l}\mathrm{C} 12 \mathrm{H} 2 \mathrm{ON} 2 \\
\mathrm{O} 3\end{array}$ & $\begin{array}{l}\text { Biosynthesis of } \\
\text { Secondary } \\
\text { Metabolites }\end{array}$ & 221737.3 & 421465.9 & 271859.1 & 306137.8 & 98345.0 & $\begin{array}{l}1640893 . \\
8\end{array}$ & 0.0 & 0.0 & 0.0 & 0.0 & 0.0 & 0.0 \\
\hline $\begin{array}{l}\mathrm{N}- \\
\text { Methylpelletierine }\end{array}$ & 155.1 & 4.7 & $\mathrm{C} 9 \mathrm{H} 17 \mathrm{NO}$ & $\begin{array}{l}\text { Biosynthesis of } \\
\text { Secondary } \\
\text { Metabolites }\end{array}$ & 98673.4 & 87350.6 & 129038.7 & 128418.8 & 181122.7 & 137681.4 & 0.0 & 0.0 & 0.0 & 0.0 & 0.0 & 0.0 \\
\hline $\begin{array}{l}\text { 5- } \\
\text { Acetamidopentano } \\
\text { ate }\end{array}$ & 159.1 & 6.9 & $\mathrm{C} 7 \mathrm{H} 13 \mathrm{NO} 3$ & $\begin{array}{l}\text { Amino Acid } \\
\text { Metabolism }\end{array}$ & 80813.3 & 182777.0 & 107368.2 & 198958.5 & 267768.3 & 126939.4 & 0.0 & 0.0 & 0.0 & 0.0 & 0.0 & 0.0 \\
\hline Phenylacetylglycine & 193.1 & 6.9 & $\begin{array}{l}\mathrm{C} 10 \mathrm{H} 11 \mathrm{NO} \\
3\end{array}$ & $\begin{array}{l}\text { Amino Acid } \\
\text { Metabolism }\end{array}$ & $\begin{array}{l}1923416 . \\
6\end{array}$ & 386052.2 & 195929.6 & $\begin{array}{l}1146176 . \\
6\end{array}$ & 298261.3 & $\begin{array}{l}3564647 . \\
5\end{array}$ & 0.0 & 0.0 & 0.0 & 0.0 & 0.0 & 0.0 \\
\hline $\begin{array}{l}\text { delta1-Piperideine- } \\
\text { 2-carboxylate }\end{array}$ & 127.1 & 7.0 & C6H9NO2 & $\begin{array}{l}\text { Amino Acid } \\
\text { Metabolism }\end{array}$ & 527432.6 & 411455.5 & 369884.8 & 568266.1 & 165638.8 & 773474.0 & 0.0 & 0.0 & 0.0 & 0.0 & 0.0 & 0.0 \\
\hline D-Aspartate & 133.0 & 14.6 & $\mathrm{C} 4 \mathrm{H} 7 \mathrm{NO} 4$ & $\begin{array}{l}\text { Amino Acid } \\
\text { Metabolism }\end{array}$ & 65609.4 & 102700.5 & 132082.6 & 219245.5 & 77002.8 & 123750.4 & 0.0 & 0.0 & 0.0 & 0.0 & 0.0 & 0.0 \\
\hline L-Glutamate & 147.1 & 14.2 & $\mathrm{C} 5 \mathrm{H} 9 \mathrm{NO} 4$ & $\begin{array}{l}\text { Amino Acid } \\
\text { Metabolism }\end{array}$ & 730015.9 & $\begin{array}{l}1226968 . \\
1\end{array}$ & 770469.3 & $\begin{array}{l}1049082 . \\
9\end{array}$ & 400025.2 & $\begin{array}{l}5106634 . \\
5\end{array}$ & $\begin{array}{l}767176 . \\
9\end{array}$ & $\begin{array}{l}2146803 . \\
8\end{array}$ & $\begin{array}{l}887554 . \\
1\end{array}$ & $\begin{array}{l}308723 . \\
0\end{array}$ & $\begin{array}{l}448335 . \\
2\end{array}$ & 469991.4 \\
\hline $\begin{array}{l}\text { (R)-2,3-Dihydroxy- } \\
\text { 3-methylbutanoate }\end{array}$ & 134.1 & 8.4 & $\mathrm{C} 5 \mathrm{H} 10 \mathrm{O} 4$ & $\begin{array}{l}\text { Amino Acid } \\
\text { Metabolism }\end{array}$ & 30540.0 & 33939.3 & 32969.0 & 30394.6 & 24138.0 & 95616.3 & 0.0 & 0.0 & 0.0 & 0.0 & 0.0 & 0.0 \\
\hline $\begin{array}{l}\text { (S)-2-Aceto-2- } \\
\text { hydroxybutanoate }\end{array}$ & 146.1 & 14.1 & $\mathrm{C} 6 \mathrm{H} 10 \mathrm{O} 4$ & $\begin{array}{l}\text { Amino Acid } \\
\text { Metabolism }\end{array}$ & 36900.5 & 25776.0 & 27845.8 & 75491.5 & 42964.1 & 50911.4 & 0.0 & 0.0 & 0.0 & 0.0 & 0.0 & 0.0 \\
\hline L-Methionine & 149.1 & 10.8 & $\begin{array}{l}\text { C5H11NO2 } \\
\text { S }\end{array}$ & $\begin{array}{l}\text { Amino Acid } \\
\text { Metabolism }\end{array}$ & 201149.0 & 143513.1 & $\begin{array}{l}1076091 . \\
4\end{array}$ & $\begin{array}{l}2039339 . \\
0\end{array}$ & $\begin{array}{l}1234755 . \\
9\end{array}$ & $\begin{array}{l}4389287 . \\
5\end{array}$ & $\begin{array}{l}162788 . \\
8\end{array}$ & 352946.3 & 57525.4 & 0.0 & 70473.7 & 0.0 \\
\hline L-Tyrosine & 181.1 & 12.4 & $\mathrm{C} 9 \mathrm{H} 11 \mathrm{NO} 3$ & $\begin{array}{l}\text { Amino Acid } \\
\text { Metabolism }\end{array}$ & 452626.6 & 740968.6 & 757670.6 & $\begin{array}{l}1127525 . \\
3\end{array}$ & 726214.3 & $\begin{array}{l}2442580 . \\
0\end{array}$ & 0.0 & 619148.3 & 0.0 & 0.0 & $\begin{array}{l}191215 . \\
8\end{array}$ & 145304.5 \\
\hline Phenylacetic acid & 136.1 & 6.9 & $\mathrm{C} 8 \mathrm{H} 8 \mathrm{O} 2$ & $\begin{array}{l}\text { Amino Acid } \\
\text { Metabolism }\end{array}$ & 88341.2 & 114739.4 & 103299.6 & 113862.0 & 29050.7 & 224081.7 & 0.0 & 0.0 & 0.0 & 0.0 & 0.0 & 0.0 \\
\hline
\end{tabular}




\begin{tabular}{|c|c|c|c|c|c|c|c|c|c|c|c|c|c|c|c|c|}
\hline L-2-Aminoadipate & 161.1 & 14.5 & C6H11NO4 & $\begin{array}{l}\text { Amino Acid } \\
\text { Metabolism }\end{array}$ & 46478.8 & 74669.9 & 79331.9 & 68681.5 & 43184.0 & 361342.0 & $\begin{array}{l}420238 \\
3.0\end{array}$ & $\begin{array}{l}4381701 . \\
5\end{array}$ & $\begin{array}{l}496351 \\
5.5\end{array}$ & $\begin{array}{l}597734 \\
0.5\end{array}$ & $\begin{array}{l}118334 \\
5.4\end{array}$ & 2676250.0 \\
\hline L-Tryptophan & 204.1 & 11.0 & $\begin{array}{l}\mathrm{C} 11 \mathrm{H} 12 \mathrm{~N} 2 \\
\mathrm{O} 2\end{array}$ & $\begin{array}{l}\text { Amino Acid } \\
\text { Metabolism }\end{array}$ & 269779.4 & 427949.8 & 436061.1 & 614904.4 & 269082.5 & $\begin{array}{l}1701747 . \\
4\end{array}$ & 0.0 & 0.0 & 0.0 & 0.0 & 0.0 & 0.0 \\
\hline 5-Oxoproline & 129.0 & 9.8 & $\mathrm{C} 5 \mathrm{H} 7 \mathrm{NO} 3$ & $\begin{array}{l}\text { Amino Acid } \\
\text { Metabolism }\end{array}$ & 372440.1 & 231566.8 & 169833.2 & $\begin{array}{l}1956034 . \\
3\end{array}$ & 186916.8 & $\begin{array}{l}1074893 . \\
9\end{array}$ & $\begin{array}{l}468761 \\
6.0\end{array}$ & $\begin{array}{l}9232550 \\
4.0\end{array}$ & $\begin{array}{l}816462 \\
5.5\end{array}$ & $\begin{array}{l}473447 \\
2.0\end{array}$ & $\begin{array}{l}631381 \\
3.0\end{array}$ & 5137753.5 \\
\hline L-Phenylalanine & 165.1 & 9.4 & $\mathrm{C9H} 11 \mathrm{NO} 2$ & $\begin{array}{l}\text { Amino Acid } \\
\text { Metabolism }\end{array}$ & $\begin{array}{l}1517887 . \\
6\end{array}$ & $\begin{array}{l}2740630 . \\
3\end{array}$ & $\begin{array}{l}2880382 . \\
3\end{array}$ & $\begin{array}{l}3722372 . \\
3\end{array}$ & $\begin{array}{l}2158948 . \\
5\end{array}$ & $\begin{array}{l}1078725 \\
3.0\end{array}$ & $\begin{array}{l}369061 . \\
4\end{array}$ & $\begin{array}{l}1527367 . \\
0\end{array}$ & $\begin{array}{l}435852 . \\
8\end{array}$ & $\begin{array}{l}634722 . \\
1\end{array}$ & $\begin{array}{l}119164 \\
3.6\end{array}$ & 770131.6 \\
\hline $\begin{array}{l}\mathrm{N}-\text { Acetyl-L- } \\
\text { citrulline }\end{array}$ & 217.1 & 11.9 & $\begin{array}{l}\mathrm{C} 8 \mathrm{H} 15 \mathrm{~N} 3 \mathrm{O} \\
4\end{array}$ & $\begin{array}{l}\text { Amino Acid } \\
\text { Metabolism }\end{array}$ & $\begin{array}{l}2400728 \\
8.0\end{array}$ & $\begin{array}{l}4963936 . \\
5\end{array}$ & $\begin{array}{l}2369858 . \\
3\end{array}$ & $\begin{array}{l}2500958 \\
2.0\end{array}$ & $\begin{array}{l}2974747 . \\
3\end{array}$ & $\begin{array}{l}9779428 \\
0.0\end{array}$ & 0.0 & 0.0 & 0.0 & 0.0 & 0.0 & 0.0 \\
\hline $\begin{array}{l}\text { 4-(2-Aminophenyl)- } \\
2,4- \\
\text { dioxobutanoate }\end{array}$ & 207.1 & 7.0 & $\mathrm{C} 10 \mathrm{H} 9 \mathrm{NO} 4$ & $\begin{array}{l}\text { Amino Acid } \\
\text { Metabolism }\end{array}$ & $\begin{array}{l}2219721 . \\
3\end{array}$ & $\begin{array}{l}2113249 . \\
0\end{array}$ & $\begin{array}{l}2433671 . \\
5\end{array}$ & $\begin{array}{l}1916869 . \\
5\end{array}$ & 917864.8 & $\begin{array}{l}4891721 . \\
5\end{array}$ & 0.0 & 0.0 & 0.0 & 0.0 & 0.0 & 0.0 \\
\hline 2-Oxosuccinamate & 131.0 & 8.7 & $\mathrm{C} 4 \mathrm{H} 5 \mathrm{NO} 4$ & $\begin{array}{l}\text { Amino Acid } \\
\text { Metabolism }\end{array}$ & 64591.8 & 51668.6 & 58934.7 & 75628.1 & 18652.0 & 178137.8 & 0.0 & 0.0 & 0.0 & 0.0 & 0.0 & 0.0 \\
\hline 5-Aminolevulinate & 131.1 & 13.7 & C5H9NO3 & $\begin{array}{l}\text { Amino Acid } \\
\text { Metabolism }\end{array}$ & 31701.5 & 0.0 & 0.0 & 30285.8 & 15474.0 & 83043.4 & 0.0 & 0.0 & 0.0 & 0.0 & 0.0 & 0.0 \\
\hline L-Threonine & 119.1 & 14.1 & C4H9NO3 & $\begin{array}{l}\text { Amino Acid } \\
\text { Metabolism }\end{array}$ & 95481.3 & 132047.2 & 149542.7 & 188663.0 & 77626.1 & 366827.5 & 0.0 & 0.0 & 0.0 & 0.0 & 0.0 & 0.0 \\
\hline Urocanate & 138.0 & 10.5 & $\mathrm{C} 6 \mathrm{H} 6 \mathrm{~N} 2 \mathrm{O} 2$ & $\begin{array}{l}\text { Amino Acid } \\
\text { Metabolism }\end{array}$ & 106431.1 & 162977.1 & 108405.3 & 932955.1 & 86048.9 & 174417.9 & $\begin{array}{l}207347 . \\
1\end{array}$ & 149112.1 & $\begin{array}{l}125300 . \\
9\end{array}$ & 48738.0 & $\begin{array}{l}138318 . \\
2\end{array}$ & 113074.5 \\
\hline $\begin{array}{l}\text { (R)-2- } \\
\text { Hydroxyglutarate }\end{array}$ & 148.0 & 15.2 & $\mathrm{C} 5 \mathrm{H} 8 \mathrm{O} 5$ & $\begin{array}{l}\text { Amino Acid } \\
\text { Metabolism }\end{array}$ & 147527.8 & 151550.5 & 234562.8 & 447816.7 & 137395.5 & 534901.8 & 0.0 & 0.0 & 0.0 & 0.0 & 0.0 & 0.0 \\
\hline L-Valine & 117.1 & 11.8 & $\mathrm{C} 5 \mathrm{H} 11 \mathrm{NO} 2$ & $\begin{array}{l}\text { Amino Acid } \\
\text { Metabolism }\end{array}$ & 148293.2 & 216106.3 & 328354.4 & 361231.4 & 184767.7 & 646574.6 & $\begin{array}{l}376518 . \\
5\end{array}$ & 775047.6 & $\begin{array}{l}227067 . \\
9\end{array}$ & $\begin{array}{l}147383 . \\
8\end{array}$ & $\begin{array}{l}191910 . \\
7\end{array}$ & 136837.0 \\
\hline $\begin{array}{l}\text { 2-Hydroxy-2,4- } \\
\text { pentadienoate }\end{array}$ & 114.0 & 14.6 & $\mathrm{C} 5 \mathrm{H} 6 \mathrm{O} 3$ & $\begin{array}{l}\text { Amino Acid } \\
\text { Metabolism }\end{array}$ & 36383.4 & 52174.6 & 59689.7 & 65515.5 & 41701.8 & 53951.8 & 0.0 & 0.0 & 0.0 & 0.0 & 0.0 & 0.0 \\
\hline N-Acetylornithine & 174.1 & 14.6 & $\begin{array}{l}\text { C7H14N2O } \\
3\end{array}$ & $\begin{array}{l}\text { Amino Acid } \\
\text { Metabolism }\end{array}$ & 34714.0 & 20363.8 & 16085.3 & 38357.7 & 11941.2 & 143732.5 & 0.0 & 0.0 & 0.0 & 0.0 & 0.0 & 0.0 \\
\hline $\begin{array}{l}\text { N(pi)-Methyl-L- } \\
\text { histidine }\end{array}$ & 169.1 & 11.6 & $\begin{array}{l}\text { C7H11N3O } \\
2\end{array}$ & $\begin{array}{l}\text { Amino Acid } \\
\text { Metabolism }\end{array}$ & 587820.7 & $\begin{array}{l}1284968 . \\
3\end{array}$ & 865524.6 & $\begin{array}{l}1033877 . \\
9\end{array}$ & 202327.0 & $\begin{array}{l}6433003 . \\
5\end{array}$ & $\begin{array}{l}115650 . \\
5\end{array}$ & 0.0 & 0.0 & 0.0 & 50920.5 & 43319.0 \\
\hline L-Proline & 115.1 & 12.0 & C5H9NO2 & $\begin{array}{l}\text { Amino Acid } \\
\text { Metabolism }\end{array}$ & 818504.8 & $\begin{array}{l}1304924 . \\
9\end{array}$ & $\begin{array}{l}1408306 . \\
0\end{array}$ & $\begin{array}{l}2553932 . \\
5\end{array}$ & $\begin{array}{l}1234451 . \\
8\end{array}$ & $\begin{array}{l}4776636 . \\
0\end{array}$ & $\begin{array}{l}316367 . \\
0\end{array}$ & 401737.7 & 65921.4 & 0.0 & $\begin{array}{l}106006 . \\
7\end{array}$ & 59772.8 \\
\hline
\end{tabular}




\begin{tabular}{|c|c|c|c|c|c|c|c|c|c|c|c|c|c|c|c|c|}
\hline L-Histidine & 155.1 & 14.3 & $\mathrm{C} 6 \mathrm{H} 9 \mathrm{~N} 3 \mathrm{O} 2$ & $\begin{array}{l}\text { Amino Acid } \\
\text { Metabolism }\end{array}$ & 533754.3 & 918863.9 & 756569.1 & 937115.8 & 261658.0 & $\begin{array}{l}1788082 . \\
6\end{array}$ & 0.0 & 0.0 & 0.0 & 0.0 & 0.0 & 0.0 \\
\hline $\begin{array}{l}\text { gamma-Glutamyl- } \\
\text { beta- } \\
\text { aminopropiononitri } \\
\text { le }\end{array}$ & 199.1 & 7.7 & $\begin{array}{l}\mathrm{C} 8 \mathrm{H} 13 \mathrm{~N} 3 \mathrm{O} \\
3\end{array}$ & $\begin{array}{l}\text { Amino Acid } \\
\text { Metabolism }\end{array}$ & 497372.2 & 52252.9 & 36452.6 & 90019.5 & 20331.5 & 104923.1 & 0.0 & 0.0 & 0.0 & 0.0 & 0.0 & 0.0 \\
\hline L-Serine & 105.0 & 15.6 & $\mathrm{C} 3 \mathrm{H} 7 \mathrm{NO} 3$ & $\begin{array}{l}\text { Amino Acid } \\
\text { Metabolism }\end{array}$ & 36947.7 & 53354.5 & 43678.7 & 211061.0 & 53041.7 & 317764.9 & 0.0 & 0.0 & 0.0 & 0.0 & 0.0 & 0.0 \\
\hline L-Leucine & 131.1 & 10.0 & $\mathrm{C} 6 \mathrm{H} 13 \mathrm{NO} 2$ & $\begin{array}{l}\text { Amino Acid } \\
\text { Metabolism }\end{array}$ & $\begin{array}{l}1652993 . \\
8\end{array}$ & $\begin{array}{l}2629253 . \\
8\end{array}$ & $\begin{array}{l}3329748 . \\
5\end{array}$ & $\begin{array}{l}3804274 . \\
0\end{array}$ & $\begin{array}{l}1955761 . \\
3\end{array}$ & $\begin{array}{l}9755629 . \\
0\end{array}$ & 0.0 & 0.0 & 0.0 & 0.0 & 0.0 & 0.0 \\
\hline L-Alanine & 89.0 & 14.1 & $\mathrm{C} 3 \mathrm{H} 7 \mathrm{NO} 2$ & $\begin{array}{l}\text { Amino Acid } \\
\text { Metabolism }\end{array}$ & 216920.5 & 238597.4 & 193331.1 & 600216.7 & 83178.2 & $\begin{array}{l}1029177 . \\
1\end{array}$ & $\begin{array}{l}492127 . \\
4\end{array}$ & $\begin{array}{l}7897206 . \\
0\end{array}$ & $\begin{array}{l}103259 \\
5.8\end{array}$ & $\begin{array}{l}550260 . \\
8\end{array}$ & $\begin{array}{l}666412 . \\
6\end{array}$ & 646077.6 \\
\hline L-Citrulline & 175.1 & 14.8 & $\begin{array}{l}\mathrm{C} 6 \mathrm{H} 13 \mathrm{~N} 3 \mathrm{O} \\
3\end{array}$ & $\begin{array}{l}\text { Amino Acid } \\
\text { Metabolism }\end{array}$ & 33888.6 & 70809.9 & 34780.2 & 318170.5 & 13050.9 & 294950.7 & 0.0 & 0.0 & 0.0 & 0.0 & 0.0 & 0.0 \\
\hline L-Glutamine & 146.1 & 14.4 & $\begin{array}{l}\mathrm{C} 5 \mathrm{H} 10 \mathrm{~N} 2 \mathrm{O} \\
3\end{array}$ & $\begin{array}{l}\text { Amino Acid } \\
\text { Metabolism }\end{array}$ & $\begin{array}{l}9028308 . \\
0\end{array}$ & $\begin{array}{l}1126611 \\
7.0\end{array}$ & $\begin{array}{l}5903626 . \\
5\end{array}$ & $\begin{array}{l}1174229 \\
6.0\end{array}$ & $\begin{array}{l}2505802 . \\
0\end{array}$ & $\begin{array}{l}4782799 \\
2.0\end{array}$ & 0.0 & 0.0 & 0.0 & 0.0 & 0.0 & 0.0 \\
\hline N6-Acetyl-L-lysine & 188.1 & 14.1 & $\begin{array}{l}\mathrm{C} 8 \mathrm{H} 16 \mathrm{~N} 2 \mathrm{O} \\
3\end{array}$ & $\begin{array}{l}\text { Amino Acid } \\
\text { Metabolism }\end{array}$ & 85663.6 & 177343.2 & 112403.1 & 169749.4 & 56842.8 & 780657.2 & 0.0 & 0.0 & 0.0 & 0.0 & 0.0 & 0.0 \\
\hline O-Acetylcarnitine & 203.1 & 9.8 & $\mathrm{C9H} 17 \mathrm{NO} 4$ & $\begin{array}{l}\text { Amino Acid } \\
\text { Metabolism }\end{array}$ & 534633.3 & $\begin{array}{l}1086755 . \\
0\end{array}$ & 403462.5 & 566083.3 & 82054.4 & $\begin{array}{l}7038791 . \\
5\end{array}$ & 0.0 & 0.0 & 0.0 & 0.0 & 0.0 & 0.0 \\
\hline Choline & 103.1 & 20.7 & $\mathrm{C} 5 \mathrm{H} 13 \mathrm{NO}$ & $\begin{array}{l}\text { Amino Acid } \\
\text { Metabolism }\end{array}$ & 245057.7 & 445097.7 & 341137.9 & 376981.4 & 146879.2 & $\begin{array}{l}1356564 . \\
1\end{array}$ & 0.0 & 0.0 & 0.0 & 0.0 & 0.0 & 0.0 \\
\hline L-Arginine & 174.1 & 24.1 & $\begin{array}{l}\mathrm{C} 6 \mathrm{H} 14 \mathrm{~N} 4 \mathrm{O} \\
2\end{array}$ & $\begin{array}{l}\text { Amino Acid } \\
\text { Metabolism }\end{array}$ & $\begin{array}{l}2415660 . \\
0\end{array}$ & $\begin{array}{l}5091256 . \\
5\end{array}$ & $\begin{array}{l}4096847 . \\
3\end{array}$ & $\begin{array}{l}4020738 \\
3\end{array}$ & $\begin{array}{l}1929505 . \\
1\end{array}$ & $\begin{array}{l}1625606 \\
9.0\end{array}$ & 0.0 & 0.0 & 0.0 & 0.0 & 0.0 & 0.0 \\
\hline L-Carnitine & 161.1 & 12.3 & $\mathrm{C} 7 \mathrm{H} 15 \mathrm{NO} 3$ & $\begin{array}{l}\text { Amino Acid } \\
\text { Metabolism }\end{array}$ & $\begin{array}{l}2104304 . \\
3\end{array}$ & $\begin{array}{l}5625441 . \\
5\end{array}$ & $\begin{array}{l}3462138 . \\
3\end{array}$ & $\begin{array}{l}3291376 . \\
8\end{array}$ & 769714.9 & $\begin{array}{l}2064694 \\
6.0\end{array}$ & 0.0 & 0.0 & 0.0 & 0.0 & 0.0 & 0.0 \\
\hline Betaine & 117.1 & 10.4 & $\mathrm{C} 5 \mathrm{H} 11 \mathrm{NO} 2$ & $\begin{array}{l}\text { Amino Acid } \\
\text { Metabolism }\end{array}$ & $\begin{array}{l}4070862 . \\
3\end{array}$ & $\begin{array}{l}8768085 . \\
0\end{array}$ & $\begin{array}{l}5432501 . \\
5\end{array}$ & $\begin{array}{l}6100318 . \\
5\end{array}$ & $\begin{array}{l}1212954 . \\
8\end{array}$ & $\begin{array}{l}4527198 \\
8.0\end{array}$ & 0.0 & 0.0 & 0.0 & 0.0 & 0.0 & 0.0 \\
\hline $\begin{array}{l}\text { 4- } \\
\text { Trimethylammonio } \\
\text { butanoate }\end{array}$ & 145.1 & 12.3 & $\mathrm{C} 7 \mathrm{H} 15 \mathrm{NO} 2$ & $\begin{array}{l}\text { Amino Acid } \\
\text { Metabolism }\end{array}$ & 369773.5 & 825234.2 & 490346.7 & 537498.1 & 117435.7 & $\begin{array}{l}2905579 . \\
5\end{array}$ & 0.0 & 0.0 & 0.0 & 0.0 & 0.0 & 0.0 \\
\hline L-Lysine & 146.1 & 22.5 & $\begin{array}{l}\mathrm{C} 6 \mathrm{H} 14 \mathrm{~N} 2 \mathrm{O} \\
2\end{array}$ & $\begin{array}{l}\text { Amino Acid } \\
\text { Metabolism }\end{array}$ & 116848.1 & 212506.2 & 265491.6 & 317387.5 & 178695.4 & 772832.1 & 0.0 & 0.0 & 0.0 & 0.0 & 0.0 & 0.0 \\
\hline
\end{tabular}




\begin{tabular}{|c|c|c|c|c|c|c|c|c|c|c|c|c|c|c|c|c|}
\hline Allysine & 145.1 & 7.6 & $\mathrm{C} 6 \mathrm{H} 11 \mathrm{NO} 3$ & $\begin{array}{l}\text { Amino Acid } \\
\text { Metabolism }\end{array}$ & 33169.6 & 48650.4 & 34509.8 & 52095.6 & 30022.1 & 137519.4 & 0.0 & 0.0 & 0.0 & 0.0 & 0.0 & 0.0 \\
\hline $\begin{array}{l}\text { N6,N6,N6- } \\
\text { Trimethyl-L-lysine }\end{array}$ & 188.2 & 21.1 & $\begin{array}{l}\mathrm{C9H} 2 \mathrm{ON} 2 \mathrm{O} \\
2\end{array}$ & $\begin{array}{l}\text { Amino Acid } \\
\text { Metabolism }\end{array}$ & 62202.8 & 175107.4 & 106606.8 & 156214.0 & 25782.3 & 777324.4 & 27802.8 & 48830.4 & 16147.0 & 9647.2 & 13870.1 & 0.0 \\
\hline $\begin{array}{l}\text { gamma-Glutamyl- } \\
\text { gamma- } \\
\text { aminobutyraldehyd } \\
\text { e }\end{array}$ & 216.1 & 8.2 & $\begin{array}{l}\mathrm{C} 9 \mathrm{H} 16 \mathrm{~N} 2 \mathrm{O} \\
4\end{array}$ & $\begin{array}{l}\text { Amino Acid } \\
\text { Metabolism }\end{array}$ & 26036.8 & 39764.6 & 19871.2 & 27242.1 & 16381.0 & 115404.7 & 0.0 & 0.0 & 0.0 & 0.0 & 0.0 & 0.0 \\
\hline (S)-2-Acetolactate & 132.0 & 14.5 & $\mathrm{C} 5 \mathrm{H} 8 \mathrm{O} 4$ & $\begin{array}{l}\text { Amino Acid } \\
\text { Metabolism }\end{array}$ & 56592.0 & 54842.7 & 66941.0 & 50435.9 & 53415.9 & 58126.7 & 0.0 & 0.0 & 0.0 & 0.0 & 0.0 & 0.0 \\
\hline L-Pipecolate & 129.1 & 11.4 & $\mathrm{C} 6 \mathrm{H} 11 \mathrm{NO} 2$ & $\begin{array}{l}\text { Amino Acid } \\
\text { Metabolism }\end{array}$ & 23636.8 & 25201.9 & 18235.4 & 17582.5 & 13714.7 & 85271.4 & 0.0 & 0.0 & 0.0 & 0.0 & 0.0 & 0.0 \\
\hline N-Acetylputrescine & 130.1 & 21.0 & $\mathrm{C} 6 \mathrm{H} 14 \mathrm{~N} 2 \mathrm{O}$ & $\begin{array}{l}\text { Amino Acid } \\
\text { Metabolism }\end{array}$ & 24972.6 & 31042.6 & 30624.5 & 33413.0 & 21747.0 & 75722.6 & 0.0 & 0.0 & 0.0 & 0.0 & 0.0 & 0.0 \\
\hline $\begin{array}{l}\text { 3,4- } \\
\text { Dihydroxyphenylac } \\
\text { etaldehyde }\end{array}$ & 152.0 & 9.5 & $\mathrm{C} 8 \mathrm{H} 8 \mathrm{O} 3$ & $\begin{array}{l}\text { Amino Acid } \\
\text { Metabolism }\end{array}$ & 32032.6 & 33122.7 & 35487.0 & 31383.5 & 19075.6 & 75688.6 & 0.0 & 0.0 & 0.0 & 0.0 & 0.0 & 0.0 \\
\hline $\begin{array}{l}\text { 5- } \\
\text { Aminopentanamid } \\
\text { e }\end{array}$ & 116.1 & 21.3 & $\mathrm{C} 5 \mathrm{H} 12 \mathrm{~N} 2 \mathrm{O}$ & $\begin{array}{l}\text { Amino Acid } \\
\text { Metabolism }\end{array}$ & 3959.0 & 4794.8 & 5984.5 & 5024.6 & 4963.8 & 6926.7 & 0.0 & 0.0 & 0.0 & 0.0 & 0.0 & 0.0 \\
\hline Homoarginine & 188.1 & 22.4 & $\begin{array}{l}\mathrm{C} 7 \mathrm{H} 16 \mathrm{~N} 4 \mathrm{O} \\
2\end{array}$ & $\begin{array}{l}\text { Amino Acid } \\
\text { Metabolism }\end{array}$ & 42892.7 & 94901.1 & 55064.5 & 69373.7 & 14051.6 & 460467.3 & 0.0 & 0.0 & 0.0 & 0.0 & 0.0 & 0.0 \\
\hline $\begin{array}{l}\text { N-Acetyl-L- } \\
\text { phenylalanine }\end{array}$ & 207.1 & 4.7 & $\begin{array}{l}\text { C11H13NO } \\
3\end{array}$ & $\begin{array}{l}\text { Amino Acid } \\
\text { Metabolism }\end{array}$ & 45495.9 & 138945.2 & 104503.5 & 87589.7 & 22501.9 & 190399.6 & 0.0 & 0.0 & 0.0 & 0.0 & 0.0 & 0.0 \\
\hline N6-Methyl-L-Iysine & 160.1 & 22.0 & $\begin{array}{l}\text { C7H16N2O } \\
2\end{array}$ & $\begin{array}{l}\text { Amino Acid } \\
\text { Metabolism }\end{array}$ & 27692.6 & 61338.8 & 42571.0 & 48074.6 & 13944.6 & 268803.6 & 28145.4 & 18471.4 & 12693.2 & 14044.5 & 16397.4 & 12463.4 \\
\hline Azelaic acid & 188.1 & 10.7 & $\mathrm{C9H} 16 \mathrm{O} 4$ & $\begin{array}{l}\text { Xenobiotics Drugs } \\
\text { etc }\end{array}$ & 0.0 & 0.0 & 0.0 & 0.0 & 0.0 & 0.0 & $\begin{array}{l}102847 . \\
9\end{array}$ & 0.0 & 0.0 & $\begin{array}{l}163590 . \\
5\end{array}$ & 0.0 & 0.0 \\
\hline Pyruvate & 88.0 & 8.3 & $\mathrm{C} 3 \mathrm{H} 4 \mathrm{O} 3$ & $\begin{array}{l}\text { Carbohydrate } \\
\text { Metabolism }\end{array}$ & 0.0 & 0.0 & 0.0 & 0.0 & 0.0 & 0.0 & $\begin{array}{l}976839 . \\
2\end{array}$ & 265698.2 & $\begin{array}{l}975265 . \\
1\end{array}$ & $\begin{array}{l}905144 . \\
1\end{array}$ & $\begin{array}{l}140779 \\
1.1\end{array}$ & 1142046.6 \\
\hline Deoxyribose & 134.1 & 7.3 & $\mathrm{C} 5 \mathrm{H} 1004$ & $\begin{array}{l}\text { Carbohydrate } \\
\text { Metabolism }\end{array}$ & 0.0 & 0.0 & 0.0 & 0.0 & 0.0 & 0.0 & $\begin{array}{l}630968 . \\
0\end{array}$ & 153740.9 & $\begin{array}{l}481546 . \\
1\end{array}$ & $\begin{array}{l}509337 . \\
2\end{array}$ & $\begin{array}{l}568936 . \\
6\end{array}$ & 455992.8 \\
\hline 2-Methylcitrate & 206.0 & 18.1 & $\mathrm{C} 7 \mathrm{H} 1007$ & $\begin{array}{l}\text { Carbohydrate } \\
\text { Metabolism }\end{array}$ & 0.0 & 0.0 & 0.0 & 0.0 & 0.0 & 0.0 & 22229.2 & 32700.6 & 0.0 & 32413.2 & 84223.5 & 39264.9 \\
\hline
\end{tabular}




\begin{tabular}{|c|c|c|c|c|c|c|c|c|c|c|c|c|c|c|c|c|}
\hline $\begin{array}{l}\text { L-1-Pyrroline-3- } \\
\text { hydroxy-5- } \\
\text { carboxylate }\end{array}$ & 129.0 & 15.7 & $\mathrm{C} 5 \mathrm{H} 7 \mathrm{NO} 3$ & $\begin{array}{l}\text { Amino Acid } \\
\text { Metabolism }\end{array}$ & 0.0 & 0.0 & 0.0 & 0.0 & 0.0 & 0.0 & $\begin{array}{l}579405 . \\
4\end{array}$ & $\begin{array}{l}5614835 . \\
0\end{array}$ & $\begin{array}{l}129202 \\
5.1\end{array}$ & $\begin{array}{l}943359 . \\
4\end{array}$ & $\begin{array}{l}262820 \\
9.5\end{array}$ & 1637015.0 \\
\hline 4-Oxoproline & 129.0 & 15.3 & $\mathrm{C} 5 \mathrm{H} 7 \mathrm{NO} 3$ & $\begin{array}{l}\text { Amino Acid } \\
\text { Metabolism }\end{array}$ & 0.0 & 0.0 & 0.0 & 0.0 & 0.0 & 0.0 & $\begin{array}{l}121898 \\
0.6\end{array}$ & $\begin{array}{l}2633423 \\
4.0\end{array}$ & $\begin{array}{l}316634 \\
2.0\end{array}$ & $\begin{array}{l}172012 \\
6.6\end{array}$ & $\begin{array}{l}220360 \\
2.3\end{array}$ & 2136488.8 \\
\hline L-Asparagine & 132.1 & 15.6 & $\mathrm{C} 4 \mathrm{H} 8 \mathrm{~N} 2 \mathrm{O} 3$ & $\begin{array}{l}\text { Amino Acid } \\
\text { Metabolism }\end{array}$ & 0.0 & 0.0 & 0.0 & 0.0 & 0.0 & 0.0 & 40175.2 & 21267.6 & 0.0 & 0.0 & 85552.2 & 24740.0 \\
\hline $\begin{array}{l}\text { 4- } \\
\text { Hydroxyphenylacet } \\
\text { aldehyde }\end{array}$ & 136.1 & 5.1 & $\mathrm{C} 8 \mathrm{H} 8 \mathrm{O} 2$ & $\begin{array}{l}\text { Amino Acid } \\
\text { Metabolism }\end{array}$ & 0.0 & 0.0 & 0.0 & 0.0 & 0.0 & 0.0 & $\begin{array}{l}270927 \\
7.8\end{array}$ & $\begin{array}{l}1986623 . \\
5\end{array}$ & $\begin{array}{l}189058 \\
7.5\end{array}$ & $\begin{array}{l}191056 \\
6.0\end{array}$ & $\begin{array}{l}326405 \\
2.5\end{array}$ & 2511199.0 \\
\hline D-Glutamate & 147.1 & 15.3 & C5H9NO4 & $\begin{array}{l}\text { Amino Acid } \\
\text { Metabolism }\end{array}$ & 0.0 & 0.0 & 0.0 & 0.0 & 0.0 & 0.0 & $\begin{array}{l}369799 . \\
6\end{array}$ & $\begin{array}{l}1603457 . \\
8\end{array}$ & $\begin{array}{l}531948 . \\
3\end{array}$ & $\begin{array}{l}115077 . \\
7\end{array}$ & $\begin{array}{l}387190 . \\
3\end{array}$ & 311705.7 \\
\hline $\begin{array}{l}\text { (R)-2,3-Dihydroxy- } \\
\text { 3- } \\
\text { methylpentanoate }\end{array}$ & 148.1 & 14.7 & $\mathrm{C} 6 \mathrm{H} 12 \mathrm{O} 4$ & $\begin{array}{l}\text { Amino Acid } \\
\text { Metabolism }\end{array}$ & 0.0 & 0.0 & 0.0 & 0.0 & 0.0 & 0.0 & 65326.4 & 512934.8 & 56706.6 & 35349.8 & 44846.6 & 39644.2 \\
\hline $\begin{array}{l}2,5- \\
\text { Dihydroxybenzoate }\end{array}$ & 154.0 & 8.3 & $\mathrm{C} 7 \mathrm{H} 6 \mathrm{O} 4$ & $\begin{array}{l}\text { Amino Acid } \\
\text { Metabolism }\end{array}$ & 0.0 & 0.0 & 0.0 & 0.0 & 0.0 & 0.0 & $\begin{array}{l}182635 . \\
5\end{array}$ & 0.0 & $\begin{array}{l}165055 . \\
6\end{array}$ & 0.0 & $\begin{array}{l}215109 . \\
2\end{array}$ & 169746.1 \\
\hline $\begin{array}{l}\text { 3,4-Dihydroxy-L- } \\
\text { phenylalanine }\end{array}$ & 197.1 & 7.5 & $\mathrm{C9H11NO4}$ & $\begin{array}{l}\text { Amino Acid } \\
\text { Metabolism }\end{array}$ & 0.0 & 0.0 & 0.0 & 0.0 & 0.0 & 0.0 & $\begin{array}{l}162877 . \\
2\end{array}$ & $\begin{array}{l}1420583 . \\
5\end{array}$ & $\begin{array}{l}312035 . \\
7\end{array}$ & $\begin{array}{l}126214 . \\
3\end{array}$ & $\begin{array}{l}176010 . \\
5\end{array}$ & 151253.3 \\
\hline $\begin{array}{l}\text { 3-(4- } \\
\text { Hydroxyphenyl)pyr } \\
\text { uvate }\end{array}$ & 180.0 & 8.1 & $\mathrm{C9H} 8 \mathrm{O} 4$ & $\begin{array}{l}\text { Amino Acid } \\
\text { Metabolism }\end{array}$ & 0.0 & 0.0 & 0.0 & 0.0 & 0.0 & 0.0 & $\begin{array}{l}367784 . \\
5\end{array}$ & 211534.0 & $\begin{array}{l}153764 . \\
1\end{array}$ & 0.0 & $\begin{array}{l}484141 . \\
2\end{array}$ & 206069.8 \\
\hline $\begin{array}{l}\text { alpha-N- } \\
\text { Phenylacetyl-L- } \\
\text { glutamine }\end{array}$ & 264.1 & 4.9 & $\begin{array}{l}\mathrm{C} 13 \mathrm{H} 16 \mathrm{~N} 2 \\
04\end{array}$ & $\begin{array}{l}\text { Amino Acid } \\
\text { Metabolism }\end{array}$ & 0.0 & 0.0 & 0.0 & 0.0 & 0.0 & 0.0 & $\begin{array}{l}967102 . \\
6\end{array}$ & $\begin{array}{l}1110881 \\
1.0\end{array}$ & $\begin{array}{l}206391 \\
3.5\end{array}$ & $\begin{array}{l}758444 . \\
5\end{array}$ & $\begin{array}{l}107959 \\
4.6\end{array}$ & 755268.9 \\
\hline
\end{tabular}


Table S2: Total putative lipids identified in the somatic extract (SE) and excretory/secretory products (ESP) of the infective third stage larva (L3) of Necator americanus.

\begin{tabular}{|c|c|c|c|c|c|c|c|c|c|c|c|c|c|c|c|c|c|}
\hline \multirow[t]{2}{*}{ Putative Lipids } & \multirow[t]{2}{*}{$\begin{array}{l}\text { Mass } \\
(m / z)\end{array}$} & \multirow{2}{*}{$\begin{array}{l}\text { Retention } \\
\text { time } \\
\text { (min) }\end{array}$} & \multirow[t]{2}{*}{ Formula } & \multirow[t]{2}{*}{ Class } & \multirow{2}{*}{$\begin{array}{l}\text { Metabolic } \\
\text { Pathway } \\
\text { (MAP) }\end{array}$} & \multicolumn{6}{|c|}{$\begin{array}{l}\text { Peak height values }(m z / r t) \text { of somatic extract (NA_SE) } \\
\text { replicates }\end{array}$} & \multicolumn{6}{|c|}{$\begin{array}{l}\text { Peak height values }(\mathrm{mz} / \mathrm{rt}) \text { of excretory/secretory products } \\
\text { (NA_ESP) replicates }\end{array}$} \\
\hline & & & & & & $\begin{array}{l}\text { NA_S } \\
\text { E_1 }\end{array}$ & $\begin{array}{l}\text { NA_S } \\
\text { E_2 }\end{array}$ & $\begin{array}{l}\text { NA_S } \\
\text { E_3 }\end{array}$ & $\begin{array}{l}\text { NA_S } \\
\text { E_4 }\end{array}$ & $\begin{array}{l}\text { NA_S } \\
\text { E_5 }\end{array}$ & $\begin{array}{l}\text { NA_S } \\
\text { E_6 }\end{array}$ & $\begin{array}{l}\text { NA_E } \\
\text { SP_1 }\end{array}$ & $\begin{array}{l}\text { NA_E } \\
\text { SP_2 }\end{array}$ & $\begin{array}{l}\text { NA_E } \\
\text { SP_3 }\end{array}$ & $\begin{array}{l}\text { NA_E } \\
\text { SP_4 }\end{array}$ & $\begin{array}{l}\text { NA_E } \\
\text { SP_5 }\end{array}$ & $\begin{array}{l}\text { NA_E } \\
\text { SP_6 }\end{array}$ \\
\hline $\begin{array}{l}\text { [FA (17:1)] 7-heptadecynoic } \\
\text { acid }\end{array}$ & 266.2 & 6.2 & $\mathrm{C} 17 \mathrm{H} 30 \mathrm{O} 2$ & $\begin{array}{l}\text { Fatty } \\
\text { Acyls }\end{array}$ & $\begin{array}{l}\text { Fatty } \\
\text { Acids and } \\
\text { Conjugates }\end{array}$ & $\begin{array}{l}1806 . \\
8\end{array}$ & 2264.0 & 3345.8 & 4311.4 & $\begin{array}{l}13410 \\
2\end{array}$ & 5538.6 & 0.0 & 0.0 & 0.0 & 0.0 & 0.0 & 0.0 \\
\hline $\begin{array}{l}{[\mathrm{FA}(18: 0)] \mathrm{N}-(9 \mathrm{Z}-} \\
\text { octadecenoyl)-ethanolamine }\end{array}$ & 325.3 & 8.1 & $\mathrm{C} 20 \mathrm{H} 39 \mathrm{NO} 2$ & $\begin{array}{l}\text { Fatty } \\
\text { Acyls }\end{array}$ & $\begin{array}{l}\text { Fatty } \\
\text { amides }\end{array}$ & $\begin{array}{l}9997 . \\
0\end{array}$ & $\begin{array}{l}17530 . \\
5\end{array}$ & $\begin{array}{l}24230 . \\
7\end{array}$ & $\begin{array}{l}28154 . \\
9\end{array}$ & $\begin{array}{l}41241 . \\
4\end{array}$ & $\begin{array}{l}18395 . \\
8\end{array}$ & 8157.3 & 8184.3 & 6921.8 & 8839.8 & 8125.0 & 9905.3 \\
\hline $\begin{array}{l}{[\text { FA }(18: 1)] 9 Z-} \\
\text { octadecenamide }\end{array}$ & 281.3 & 6.6 & $\mathrm{C} 18 \mathrm{H} 35 \mathrm{NO}$ & $\begin{array}{l}\text { Fatty } \\
\text { Acyls }\end{array}$ & $\begin{array}{l}\text { Fatty } \\
\text { amides }\end{array}$ & $\begin{array}{l}11620 \\
41.0\end{array}$ & $\begin{array}{l}864015 \\
.6\end{array}$ & $\begin{array}{l}145372 \\
2.9\end{array}$ & $\begin{array}{l}214960 \\
9.3\end{array}$ & $\begin{array}{l}176186 \\
5.1\end{array}$ & $\begin{array}{l}144486 \\
1.9\end{array}$ & $\begin{array}{l}926182 \\
.1\end{array}$ & $\begin{array}{l}649913 \\
.4\end{array}$ & $\begin{array}{l}804704 \\
.4\end{array}$ & $\begin{array}{l}682296 \\
.7\end{array}$ & $\begin{array}{l}299912 \\
0.3\end{array}$ & $\begin{array}{l}585068 \\
.7\end{array}$ \\
\hline $\begin{array}{l}\text { [FA (21:2)] octadecenoic } \\
\text { Acid }\end{array}$ & 324.3 & 8.6 & $\mathrm{C} 21 \mathrm{H} 40 \mathrm{O} 2$ & $\begin{array}{l}\text { Fatty } \\
\text { Acyls }\end{array}$ & $\begin{array}{l}\text { Fatty } \\
\text { Acids and } \\
\text { Conjugates }\end{array}$ & 0.0 & 0.0 & 0.0 & 0.0 & 0.0 & 0.0 & $\begin{array}{l}221637 \\
.2\end{array}$ & $\begin{array}{l}193088 \\
.1\end{array}$ & $\begin{array}{l}203702 \\
.4\end{array}$ & $\begin{array}{l}205467 \\
.5\end{array}$ & $\begin{array}{l}166596 \\
.8\end{array}$ & $\begin{array}{l}167879 \\
.6\end{array}$ \\
\hline $\begin{array}{l}{[\mathrm{FA}(22: 4)]} \\
7 Z_{-} 10 \mathrm{Z} \_13 Z_{1} 16 \mathrm{Z}- \\
\text { docosatetraenoic acid }\end{array}$ & 332.3 & 8.0 & $\mathrm{C} 22 \mathrm{H} 36 \mathrm{O} 2$ & $\begin{array}{l}\text { Fatty } \\
\text { Acyls }\end{array}$ & $\begin{array}{l}\text { Fatty } \\
\text { Acids and } \\
\text { Conjugates }\end{array}$ & $\begin{array}{l}10954 \\
.8\end{array}$ & $\begin{array}{l}16765 . \\
1\end{array}$ & $\begin{array}{l}30144 \\
7\end{array}$ & $\begin{array}{l}31566 . \\
6\end{array}$ & $\begin{array}{l}56761 . \\
9\end{array}$ & $\begin{array}{l}21704 \\
4\end{array}$ & 0.0 & 0.0 & 0.0 & 0.0 & 0.0 & 0.0 \\
\hline [FA $(23: 0)]$ tricosanoic acid & 354.4 & 12.0 & $\mathrm{C} 23 \mathrm{H} 46 \mathrm{O} 2$ & $\begin{array}{l}\text { Fatty } \\
\text { Acyls }\end{array}$ & $\begin{array}{l}\text { Fatty } \\
\text { Acids and } \\
\text { Conjugates }\end{array}$ & $\begin{array}{l}10794 \\
5.1\end{array}$ & $\begin{array}{l}142976 \\
.4\end{array}$ & $\begin{array}{l}212542 \\
.7\end{array}$ & $\begin{array}{l}261747 \\
.6\end{array}$ & $\begin{array}{l}275267 \\
.7\end{array}$ & $\begin{array}{l}207632 \\
.1\end{array}$ & 0.0 & 0.0 & 0.0 & 0.0 & 0.0 & 0.0 \\
\hline [FA (24:1)] 15-tetracosenal & 367.4 & 11.7 & $\mathrm{C} 24 \mathrm{H} 46 \mathrm{O}$ & $\begin{array}{l}\text { Fatty } \\
\text { Acyls }\end{array}$ & $\begin{array}{l}\text { Fatty } \\
\text { Acids and } \\
\text { Conjugates }\end{array}$ & 0.0 & 0.0 & 0.0 & 0.0 & 0.0 & 0.0 & $\begin{array}{l}58127 . \\
1\end{array}$ & $\begin{array}{l}52001 . \\
9\end{array}$ & $\begin{array}{l}89373 . \\
5\end{array}$ & $\begin{array}{l}40652 . \\
3\end{array}$ & $\begin{array}{l}107404 \\
.7\end{array}$ & $\begin{array}{l}36097 . \\
6\end{array}$ \\
\hline $\begin{array}{l}\text { [FA }(25: 0)] \text { pentacosanoic } \\
\text { acid }\end{array}$ & 382.4 & 13.4 & $\mathrm{C} 25 \mathrm{H} 50 \mathrm{O} 2$ & $\begin{array}{l}\text { Fatty } \\
\text { Acyls }\end{array}$ & $\begin{array}{l}\text { Fatty } \\
\text { Acids and } \\
\text { Conjugates }\end{array}$ & $\begin{array}{l}10268 \\
9.2\end{array}$ & $\begin{array}{l}99393 . \\
9\end{array}$ & $\begin{array}{l}136599 \\
.7\end{array}$ & $\begin{array}{l}174331 \\
.6\end{array}$ & $\begin{array}{l}182891 \\
.1\end{array}$ & $\begin{array}{l}166507 \\
.6\end{array}$ & 0.0 & 0.0 & 0.0 & 0.0 & 0.0 & 0.0 \\
\hline
\end{tabular}




\begin{tabular}{|c|c|c|c|c|c|c|c|c|c|c|c|c|c|c|c|c|c|}
\hline $\begin{array}{l}\text { [FA (26:0)] hexacosanoic } \\
\text { acid }\end{array}$ & 396.4 & 14.2 & $\mathrm{C} 26 \mathrm{H} 52 \mathrm{O} 2$ & $\begin{array}{l}\text { Fatty } \\
\text { Acyls }\end{array}$ & $\begin{array}{l}\text { Fatty } \\
\text { Acids and } \\
\text { Conjugates }\end{array}$ & $\begin{array}{l}43020 \\
8.8\end{array}$ & $\begin{array}{l}477406 \\
.2\end{array}$ & $\begin{array}{l}761073 \\
.3\end{array}$ & $\begin{array}{l}835973 \\
.9\end{array}$ & $\begin{array}{l}638703 \\
.1\end{array}$ & $\begin{array}{l}744810 \\
.6\end{array}$ & 0.0 & 0.0 & 0.0 & 0.0 & 0.0 & 0.0 \\
\hline $\begin{array}{l}{[\mathrm{FA}(26: 0 / 2: 0)]} \\
\text { Hexacosanedioic acid }\end{array}$ & 426.4 & 10.9 & $\mathrm{C} 26 \mathrm{H} 50 \mathrm{O} 4$ & $\begin{array}{l}\text { Fatty } \\
\text { Acyls }\end{array}$ & $\begin{array}{l}\text { Fatty } \\
\text { Acids and } \\
\text { Conjugates }\end{array}$ & $\begin{array}{l}47057 \\
.9\end{array}$ & $\begin{array}{l}38465 \\
1\end{array}$ & $\begin{array}{l}49757 . \\
1\end{array}$ & $\begin{array}{l}55476 . \\
9\end{array}$ & $\begin{array}{l}52239 . \\
2\end{array}$ & $\begin{array}{l}61304 . \\
9\end{array}$ & 0.0 & 0.0 & 0.0 & 0.0 & 0.0 & 0.0 \\
\hline $\begin{array}{l}\text { [FA (27:0)] heptacosanoic } \\
\text { acid }\end{array}$ & 427.4 & 16.5 & $\mathrm{C} 27 \mathrm{H} 54 \mathrm{O} 2$ & $\begin{array}{l}\text { Fatty } \\
\text { Acyls }\end{array}$ & $\begin{array}{l}\text { Fatty } \\
\text { Acids and } \\
\text { Conjugates }\end{array}$ & $\begin{array}{l}74577 \\
.8\end{array}$ & $\begin{array}{l}71138 . \\
9\end{array}$ & $\begin{array}{l}100214 \\
.6\end{array}$ & $\begin{array}{l}123933 \\
.8\end{array}$ & $\begin{array}{l}103599 \\
.6\end{array}$ & $\begin{array}{l}105605 \\
.6\end{array}$ & 0.0 & 0.0 & 0.0 & 0.0 & 0.0 & 0.0 \\
\hline [FA (28:0)] octacosanal & 425.5 & 16.5 & $\mathrm{C} 28 \mathrm{H} 56 \mathrm{O}$ & $\begin{array}{l}\text { Fatty } \\
\text { Acyls }\end{array}$ & $\begin{array}{l}\text { Fatty } \\
\text { aldehydes }\end{array}$ & $\begin{array}{l}79454 \\
.3\end{array}$ & $\begin{array}{l}131399 \\
.4\end{array}$ & $\begin{array}{l}60197 . \\
6\end{array}$ & $\begin{array}{l}112325 \\
.3\end{array}$ & $\begin{array}{l}33725 \\
6\end{array}$ & $\begin{array}{l}39463 . \\
7\end{array}$ & 5831.5 & 5227.9 & 5220.3 & 4424.4 & 5113.0 & 8003.5 \\
\hline [FA (28:0)] octacosanoic acid & 424.4 & 15.8 & $\mathrm{C} 28 \mathrm{H} 56 \mathrm{O} 2$ & $\begin{array}{l}\text { Fatty } \\
\text { Acyls }\end{array}$ & $\begin{array}{l}\text { Fatty } \\
\text { Acids and } \\
\text { Conjugates }\end{array}$ & $\begin{array}{l}43354 \\
.3\end{array}$ & $\begin{array}{l}42455 . \\
1\end{array}$ & $\begin{array}{l}68853 . \\
1\end{array}$ & $\begin{array}{l}86625 \\
7\end{array}$ & $\begin{array}{l}55090 \\
8\end{array}$ & $\begin{array}{l}72357 . \\
7\end{array}$ & 0.0 & 0.0 & 0.0 & 0.0 & 0.0 & 0.0 \\
\hline $\begin{array}{l}\text { [FA (29:0)] nonacosanoic } \\
\text { acid }\end{array}$ & 455.5 & 18.3 & $\mathrm{C} 29 \mathrm{H} 58 \mathrm{O} 2$ & $\begin{array}{l}\text { Fatty } \\
\text { Acyls }\end{array}$ & $\begin{array}{l}\text { Fatty } \\
\text { Acids and } \\
\text { Conjugates }\end{array}$ & $\begin{array}{l}51483 \\
.0\end{array}$ & $\begin{array}{l}78871 . \\
7\end{array}$ & $\begin{array}{l}25451 . \\
8\end{array}$ & $\begin{array}{l}40623 . \\
1\end{array}$ & $\begin{array}{l}14183 . \\
4\end{array}$ & $\begin{array}{l}47369 . \\
6\end{array}$ & 0.0 & 0.0 & 0.0 & 0.0 & 0.0 & 0.0 \\
\hline $\begin{array}{l}\text { [FA (31:0)] hentriacontanoic } \\
\text { acid }\end{array}$ & 483.5 & 19.7 & $\mathrm{C} 31 \mathrm{H} 62 \mathrm{O} 2$ & $\begin{array}{l}\text { Fatty } \\
\text { Acyls }\end{array}$ & $\begin{array}{l}\text { Fatty } \\
\text { Acids and } \\
\text { Conjugates }\end{array}$ & $\begin{array}{l}18429 \\
.3\end{array}$ & $\begin{array}{l}26062 . \\
9\end{array}$ & $\begin{array}{l}16005 \\
8\end{array}$ & $\begin{array}{l}13462 . \\
6\end{array}$ & 4745.2 & $\begin{array}{l}30591 . \\
8\end{array}$ & 0.0 & 0.0 & 0.0 & 0.0 & 0.0 & 0.0 \\
\hline $\begin{array}{l}\text { [FA hydroxy(11:1)] } \\
\text { undecenoic acid }\end{array}$ & 200.1 & 1.5 & $\mathrm{C} 11 \mathrm{H} 20 \mathrm{O} 3$ & $\begin{array}{l}\text { Fatty } \\
\text { Acyls }\end{array}$ & $\begin{array}{l}\text { Fatty } \\
\text { Acids and } \\
\text { Conjugates }\end{array}$ & $\begin{array}{l}29106 \\
.9\end{array}$ & $\begin{array}{l}25139 . \\
5\end{array}$ & $\begin{array}{l}28641 . \\
8\end{array}$ & $\begin{array}{l}20318 \\
1\end{array}$ & $\begin{array}{l}23852 . \\
9\end{array}$ & $\begin{array}{l}57711 . \\
6\end{array}$ & 0.0 & 0.0 & 0.0 & 0.0 & 0.0 & 0.0 \\
\hline $\begin{array}{l}{[\text { FA hydroxy }(30: 0)] 30-} \\
\text { hydroxy-triacontanoic acid }\end{array}$ & 485.5 & 14.7 & $\mathrm{C} 30 \mathrm{H} 60 \mathrm{O} 3$ & $\begin{array}{l}\text { Fatty } \\
\text { Acyls }\end{array}$ & $\begin{array}{l}\text { Fatty } \\
\text { Acids and } \\
\text { Conjugates }\end{array}$ & $\begin{array}{l}28465 \\
4.4\end{array}$ & $\begin{array}{l}559611 \\
.4\end{array}$ & $\begin{array}{l}328418 \\
.5\end{array}$ & $\begin{array}{l}545707 \\
.1\end{array}$ & $\begin{array}{l}159261 \\
.4\end{array}$ & $\begin{array}{l}336945 \\
.8\end{array}$ & 0.0 & 0.0 & 0.0 & 0.0 & 0.0 & 0.0 \\
\hline $\begin{array}{l}{[\text { FA trimethyl }(13: 2)]} \\
\text { tridecadienoic acid }\end{array}$ & 266.2 & 2.9 & $\mathrm{C} 16 \mathrm{H} 26 \mathrm{O} 3$ & $\begin{array}{l}\text { Fatty } \\
\text { Acyls }\end{array}$ & $\begin{array}{l}\text { Fatty } \\
\text { Acids and } \\
\text { Conjugates }\end{array}$ & 0.0 & 0.0 & 0.0 & 0.0 & 0.0 & 0.0 & $\begin{array}{l}100211 \\
.3\end{array}$ & $\begin{array}{l}114593 \\
.3\end{array}$ & $\begin{array}{l}72224 . \\
2\end{array}$ & $\begin{array}{l}81198 . \\
4\end{array}$ & $\begin{array}{l}115321 \\
.6\end{array}$ & $\begin{array}{l}92879 . \\
5\end{array}$ \\
\hline $\begin{array}{l}{[\text { Fv Methox] 4'- }} \\
\text { Methoxychalcone }\end{array}$ & 238.1 & 2.0 & $\mathrm{C} 16 \mathrm{H} 14 \mathrm{O} 2$ & $\begin{array}{l}\text { Polyketid } \\
\text { es }\end{array}$ & Flavonoids & 0.0 & 0.0 & 0.0 & 0.0 & 0.0 & 0.0 & $\begin{array}{l}825989 \\
.1\end{array}$ & $\begin{array}{l}672710 \\
.3\end{array}$ & $\begin{array}{l}548765 \\
.7\end{array}$ & $\begin{array}{l}691165 \\
.4\end{array}$ & $\begin{array}{l}758028 \\
.7\end{array}$ & $\begin{array}{l}591974 \\
.0\end{array}$ \\
\hline [Fv] Dichamanetin & 468.2 & 2.0 & $\mathrm{C} 29 \mathrm{H} 24 \mathrm{O} 6$ & $\begin{array}{l}\text { Polyketid } \\
\text { es }\end{array}$ & Flavonoids & 0.0 & 0.0 & 0.0 & 0.0 & 0.0 & 0.0 & $\begin{array}{l}105639 \\
.6\end{array}$ & $\begin{array}{l}168770 \\
.6\end{array}$ & $\begin{array}{l}164010 \\
.0\end{array}$ & $\begin{array}{l}167239 \\
.3\end{array}$ & $\begin{array}{l}132659 \\
.3\end{array}$ & $\begin{array}{l}152289 \\
.4\end{array}$ \\
\hline $\begin{array}{l}{[\text { LysoPC(14:0)] 1- }} \\
\text { tetradecanoyl-sn-glycero-3- } \\
\text { phosphocholine }\end{array}$ & 467.3 & 5.1 & $\mathrm{C} 22 \mathrm{H} 46 \mathrm{NO} 7 \mathrm{P}$ & $\begin{array}{l}\text { Glyceroph } \\
\text { ospholipid } \\
\text { s }\end{array}$ & $\begin{array}{l}\text { Glyceroph } \\
\text { osphocholi } \\
\text { nes }\end{array}$ & $\begin{array}{l}37761 \\
7.3\end{array}$ & $\begin{array}{l}714196 \\
.7\end{array}$ & $\begin{array}{l}957997 \\
.4\end{array}$ & $\begin{array}{l}959033 \\
.2\end{array}$ & $\begin{array}{l}116813 \\
7.5\end{array}$ & $\begin{array}{l}489706 \\
.8\end{array}$ & 0.0 & 0.0 & 0.0 & 0.0 & 0.0 & 0.0 \\
\hline
\end{tabular}


[LysoPC(14:1)] 1-(9Z-

tetradecenoyl)-sn-glycero-3-

phosphocholine

[LysoPC(16:2)] 1-hexadecyl-

sn-glycero-3-phosphocholine

[PA(12:0/12:0)] 1_2-

didodecanoyl-sn-glycero-3-

phosphate

[PC(11:1/11:1)] 1-(10E-

undecenoyl)-2-(10E-

undecenoyl)-sn-glycero-3-

phosphocholine

[PC(16:2)] 1-(9E_10E-

hexadecadienyl)-sn-glycero-

3-phosphocholine

[PC(18:3)]

[PC(19:3)] 1-(10Z_13Z_16Z nonadecatrienoyl)-sn-glycero-

3-phosphocholine

[PC(20:5)] 1-

(5Z_8Z_11Z_14Z_17Z

eicosapentaenoyl)-sn-glycero-

3-phosphocholine

[PC(22:3)]

$591.4 \quad 6.7$

C22H44NO7P

Glyceroph Glyceroph ospholipid osphocholi

25
7.8

nes

C24H52NO6P

Glycerop

ospholipid

44723

C27H53O8P

Glyceroph Glyceroph

ospholipid osphocholi

nes

C30H56NO8P

Glyceroph Glyceroph

ospholipid osphocholi 8

$\mathrm{s}$ nes

\section{C24H48NO6P}

Glyceroph Glyceroph$$
\text { ospholipid }
$$

Glyceroph

12760. 2

8314. 3

661. 37666

11911
5

C26H48NO7P

Glyceroph

$$
\mathrm{s}
$$

Glyceroph

9424

$8625.0 \quad 9943$

3.3 10849. 14588. $7653.1 \quad 0.0$

nes

Glyceroph Glyceroph

ospholipid osphocholi

C28H48NO7P

Glyceroph Glyceroph

ospholipid$$
\text { nes }
$$

0.0

$40.3 \quad 0.0$

0.0

0.0

0.0

0.0

$$
\text { es }
$$

Glyceroph Glyceroph 3094 ospholipid osphocholi $\quad .4$

$$
\begin{array}{ll}
\mathrm{s} & \text { nes }
\end{array}
$$




\begin{tabular}{|c|c|c|c|c|c|c|c|c|c|c|c|c|c|c|c|c|c|}
\hline$[\mathrm{PE}(12: 0)$ & 411.2 & 1.5 & C17H34NO8P & $\begin{array}{l}\text { Glyceroph } \\
\text { ospholipid } \\
\mathrm{s}\end{array}$ & $\begin{array}{l}\text { Glyceroph } \\
\text { osphocholi } \\
\text { nes }\end{array}$ & $\begin{array}{l}27642 \\
.6\end{array}$ & 4043.4 & 0.0 & 0.0 & 0.0 & 0.0 & 0.0 & 0.0 & 0.0 & 0.0 & 0.0 & 0.0 \\
\hline $\begin{array}{l}{[\mathrm{PE}(16: 1)] \text { 1-(1Z- }} \\
\text { hexadecenyl)-sn-glycero-3- } \\
\text { phosphoethanolamine }\end{array}$ & 437.3 & 5.2 & C21H44NO6P & $\begin{array}{l}\text { Glyceroph } \\
\text { ospholipid } \\
\mathrm{s}\end{array}$ & $\begin{array}{l}\text { Glyceroph } \\
\text { osphoetha } \\
\text { nolamines }\end{array}$ & $\begin{array}{l}15271 \\
.3\end{array}$ & $\begin{array}{l}16318 . \\
0\end{array}$ & $\begin{array}{l}28390 \\
2\end{array}$ & $\begin{array}{l}32737 . \\
6\end{array}$ & $\begin{array}{l}29981 . \\
4\end{array}$ & $\begin{array}{l}15261 . \\
1\end{array}$ & 0.0 & 0.0 & 0.0 & 0.0 & 0.0 & 0.0 \\
\hline $\begin{array}{l}{[\mathrm{PE}(\mathrm{O}-18: 2)] \text { 1-octadecyl-sn- }} \\
\text { glycero-3- } \\
\text { phosphoethanolamine }\end{array}$ & 467.3 & 6.4 & $\mathrm{C} 23 \mathrm{H} 50 \mathrm{NO} 6 \mathrm{P}$ & $\begin{array}{l}\text { Glyceroph } \\
\text { ospholipid } \\
\text { s }\end{array}$ & $\begin{array}{l}\text { Glyceroph } \\
\text { osphoetha } \\
\text { nolamines }\end{array}$ & $\begin{array}{l}90268 \\
3.5\end{array}$ & $\begin{array}{l}188731 \\
7.4\end{array}$ & $\begin{array}{l}258118 \\
7.3\end{array}$ & $\begin{array}{l}336149 \\
2.0\end{array}$ & $\begin{array}{l}676978 \\
2.0\end{array}$ & $\begin{array}{l}183326 \\
0.9\end{array}$ & 5327.1 & 0.0 & 2967.7 & 0.0 & 0.0 & 0.0 \\
\hline $\begin{array}{l}\text { [PG hydrox] 1-(3- } \\
\text { hydroxyphytanyl)-2-phytanyl- } \\
\text { sn-glycero-3-phospho-(1'-sn- } \\
\text { glycerol) }\end{array}$ & 822.7 & 21.0 & C46H95O9P & $\begin{array}{l}\text { Glyceroph } \\
\text { ospholipid } \\
\text { s }\end{array}$ & $\begin{array}{l}\text { Glyceroph } \\
\text { osphoglyc } \\
\text { erols }\end{array}$ & $\begin{array}{l}30768 \\
.1\end{array}$ & $\begin{array}{l}39471 . \\
7\end{array}$ & $\begin{array}{l}19113 . \\
4\end{array}$ & $\begin{array}{l}64897 . \\
6\end{array}$ & $\begin{array}{l}41585 . \\
0\end{array}$ & $\begin{array}{l}49899 . \\
4\end{array}$ & 0.0 & 0.0 & 0.0 & 0.0 & 0.0 & 0.0 \\
\hline$[\mathrm{PI}(36: 0)$ & 879.6 & 10.8 & $\begin{array}{l}\text { C45H86NO13 } \\
\text { P }\end{array}$ & $\begin{array}{l}\text { Glyceroph } \\
\text { ospholipid } \\
\mathrm{s}\end{array}$ & $\begin{array}{l}\text { Glyceroph } \\
\text { osphoinosi } \\
\text { tols }\end{array}$ & $\begin{array}{l}19776 \\
.5\end{array}$ & $\begin{array}{l}30211 \\
5\end{array}$ & $\begin{array}{l}36854 \\
7\end{array}$ & $\begin{array}{l}44412 . \\
2\end{array}$ & $\begin{array}{l}91493 . \\
0\end{array}$ & $\begin{array}{l}33172 . \\
8\end{array}$ & 0.0 & 0.0 & 0.0 & 0.0 & 0.0 & 0.0 \\
\hline $\begin{array}{l}{[\mathrm{PR}] 32 \text { 35- }} \\
\text { anhydrobacteriohopaneterol }\end{array}$ & 528.5 & 16.5 & $\mathrm{C} 35 \mathrm{H} 60 \mathrm{O} 3$ & Prenols & Hopanoids & $\begin{array}{l}67829 \\
.7\end{array}$ & $\begin{array}{l}40992 . \\
4\end{array}$ & $\begin{array}{l}119379 \\
.7\end{array}$ & $\begin{array}{l}186692 \\
.6\end{array}$ & $\begin{array}{l}36996 . \\
4\end{array}$ & $\begin{array}{l}107135 \\
.4\end{array}$ & $\begin{array}{l}184481 \\
.7\end{array}$ & $\begin{array}{l}76808 . \\
8\end{array}$ & $\begin{array}{l}514321 \\
.4\end{array}$ & $\begin{array}{l}117268 \\
.3\end{array}$ & $\begin{array}{l}92653 \\
7\end{array}$ & $\begin{array}{l}153661 \\
.0\end{array}$ \\
\hline $\begin{array}{l}{[\mathrm{PR}] 35-} \\
\text { aminobacteriohopane- } \\
\text { 32_33_34-triol }\end{array}$ & 591.5 & 8.8 & $\mathrm{C} 35 \mathrm{H} 63 \mathrm{NO} 3$ & Prenols & Hopanoids & $\begin{array}{l}11658 \\
9.2\end{array}$ & $\begin{array}{l}117939 \\
.8\end{array}$ & $\begin{array}{l}137802 \\
.3\end{array}$ & $\begin{array}{l}134879 \\
.0\end{array}$ & $\begin{array}{l}141491 \\
.6\end{array}$ & $\begin{array}{l}130310 \\
.8\end{array}$ & $\begin{array}{l}103340 \\
.2\end{array}$ & $\begin{array}{l}112622 \\
.8\end{array}$ & $\begin{array}{l}115252 \\
.3\end{array}$ & $\begin{array}{l}110724 \\
.6\end{array}$ & $\begin{array}{l}117187 \\
.4\end{array}$ & $\begin{array}{l}106962 \\
.0\end{array}$ \\
\hline $\begin{array}{l}\text { [PR] bacteriohopane- } \\
\text { 32_33_34-triol-35-cyclitol }\end{array}$ & 707.5 & 15.9 & $\mathrm{C} 41 \mathrm{H} 73 \mathrm{NO} 8$ & Prenols & Hopanoids & $\begin{array}{l}12080 \\
4.8\end{array}$ & $\begin{array}{l}165179 \\
.4\end{array}$ & $\begin{array}{l}167874 \\
.9\end{array}$ & $\begin{array}{l}174425 \\
.2\end{array}$ & $\begin{array}{l}34902 . \\
6\end{array}$ & $\begin{array}{l}143432 \\
.4\end{array}$ & 0.0 & 0.0 & 0.0 & 0.0 & 0.0 & 0.0 \\
\hline$[\mathrm{PR}]$ Beta-isorenieratane & 574.5 & 11.7 & $\mathrm{C} 40 \mathrm{H} 72$ & Prenols & $\begin{array}{l}\text { Isoprenoid } \\
\mathrm{s}\end{array}$ & $\begin{array}{l}22544 \\
1.8\end{array}$ & $\begin{array}{l}174708 \\
.3\end{array}$ & $\begin{array}{l}716359 \\
.0\end{array}$ & $\begin{array}{l}815792 \\
.8\end{array}$ & $\begin{array}{l}365068 \\
.4\end{array}$ & $\begin{array}{l}472305 \\
.5\end{array}$ & 0.0 & 0.0 & 0.0 & 0.0 & 0.0 & 0.0 \\
\hline $\begin{array}{l}{[\mathrm{PR}] \text { Tretinoin/All-Trans }} \\
\text { Retinoic Acid }\end{array}$ & 300.2 & 5.5 & $\mathrm{C} 20 \mathrm{H} 28 \mathrm{O} 2$ & Prenols & Hopanoids & $\begin{array}{l}1986 . \\
1\end{array}$ & 3094.2 & 3659.4 & $\begin{array}{l}11010 . \\
0\end{array}$ & 6047.2 & 0.0 & 0.0 & 7552.6 & 3791.4 & 2978.9 & 2426.3 & 3703.7 \\
\hline $\begin{array}{l}\text { [SP (12:0)] N-(dodecanoyl)- } \\
\text { sphing-4-enine-1-phosphate } \\
\text { (ammonium salt) }\end{array}$ & 578.4 & 7.3 & $\begin{array}{l}\mathrm{C} 30 \mathrm{H} 63 \mathrm{~N} 2 \mathrm{O} 6 \\
\mathrm{P}\end{array}$ & $\begin{array}{l}\text { Sphingoli } \\
\text { pids }\end{array}$ & Ceramides & $\begin{array}{l}98881 \\
.1\end{array}$ & $\begin{array}{l}81838 . \\
1\end{array}$ & $\begin{array}{l}179104 \\
.3\end{array}$ & $\begin{array}{l}211766 \\
.7\end{array}$ & $\begin{array}{l}192432 \\
.8\end{array}$ & $\begin{array}{l}178263 \\
.0\end{array}$ & $\begin{array}{l}104366 \\
.4\end{array}$ & $\begin{array}{l}98498 . \\
8\end{array}$ & $\begin{array}{l}90806 . \\
8\end{array}$ & $\begin{array}{l}87846 . \\
3\end{array}$ & $\begin{array}{l}119743 \\
.2\end{array}$ & $\begin{array}{l}73583 . \\
9\end{array}$ \\
\hline
\end{tabular}




\begin{tabular}{|c|c|c|c|c|c|c|c|c|c|c|c|c|c|c|c|c|c|}
\hline $\begin{array}{l}\text { [SP (16:0)] N- } \\
\text { (hexadecanoyl)-sphing-4- } \\
\text { enine }\end{array}$ & 578.4 & 7.3 & $\begin{array}{l}\text { C30H63N2O6 } \\
\text { P }\end{array}$ & $\begin{array}{l}\text { Sphingoli } \\
\text { pids }\end{array}$ & na & 0.0 & 0.0 & 0.0 & $\begin{array}{l}12002 . \\
3\end{array}$ & 0.0 & 0.0 & 0.0 & 0.0 & 0.0 & 0.0 & 0.0 & 0.0 \\
\hline $\begin{array}{l}\text { [SP } \\
\text { hydroxy_methyl_methyl(20:0 } \\
\text { )] }\end{array}$ & 369.4 & 6.6 & $\mathrm{C} 23 \mathrm{H} 47 \mathrm{NO} 2$ & $\begin{array}{l}\text { Sphingoli } \\
\text { pids }\end{array}$ & na & $\begin{array}{l}6327 . \\
9\end{array}$ & 5923.6 & 9881.4 & $\begin{array}{l}12016 . \\
0\end{array}$ & $\begin{array}{l}15451 \\
2\end{array}$ & $\begin{array}{l}11466 . \\
1\end{array}$ & $\begin{array}{l}11424 . \\
4\end{array}$ & $\begin{array}{l}10097 . \\
6\end{array}$ & 7990.6 & $\begin{array}{l}12553 . \\
7\end{array}$ & $\begin{array}{l}12997 . \\
6\end{array}$ & 7424.5 \\
\hline $\begin{array}{l}\text { [SP methyl(2:0)] 9-methyl- } \\
\text { sphinga-4E_8E-dienine }\end{array}$ & 311.3 & 8.5 & $\mathrm{C} 19 \mathrm{H} 37 \mathrm{NO} 2$ & $\begin{array}{l}\text { Sphingoli } \\
\text { pids }\end{array}$ & $\begin{array}{l}\text { Sphingoid } \\
\text { bases }\end{array}$ & $\begin{array}{l}6651 . \\
2\end{array}$ & 7097.3 & 9302.5 & $\begin{array}{l}30658 . \\
4\end{array}$ & $\begin{array}{l}11836 \\
2\end{array}$ & $\begin{array}{l}24693 \\
2\end{array}$ & 0.0 & 0.0 & 0.0 & 0.0 & 0.0 & 0.0 \\
\hline $\begin{array}{l}\text { [ST }(15: 0)] \text { cholest- } 5 \text {-en-3b-yl } \\
\text { (pentadecanoate) }\end{array}$ & 610.6 & 20.2 & $\mathrm{C} 42 \mathrm{H} 74 \mathrm{O} 2$ & $\begin{array}{l}\text { Sterol } \\
\text { lipids }\end{array}$ & Sterols & $\begin{array}{l}21396 \\
.3\end{array}$ & $\begin{array}{l}24712 . \\
6\end{array}$ & $\begin{array}{l}27196 . \\
2\end{array}$ & 0.0 & $\begin{array}{l}18769 . \\
5\end{array}$ & $\begin{array}{l}20950 . \\
6\end{array}$ & $\begin{array}{l}19179 . \\
1\end{array}$ & $\begin{array}{l}17448 . \\
0\end{array}$ & $\begin{array}{l}18567 . \\
8\end{array}$ & $\begin{array}{l}19331 . \\
4\end{array}$ & $\begin{array}{l}20219 . \\
3\end{array}$ & $\begin{array}{l}18813 . \\
8\end{array}$ \\
\hline $\begin{array}{l}\text { [ST (17:0)] cholest-5-en-3b-yl } \\
\text { (heptadecanoate) }\end{array}$ & 638.6 & 20.9 & $\mathrm{C} 44 \mathrm{H} 78 \mathrm{O} 2$ & $\begin{array}{l}\text { Sterol } \\
\text { lipids }\end{array}$ & Sterols & 0.0 & 0.0 & 0.0 & 0.0 & 0.0 & 0.0 & 9111.2 & 9190.6 & $\begin{array}{l}10372 . \\
9\end{array}$ & 8641.7 & 7254.7 & $\begin{array}{l}10560 . \\
0\end{array}$ \\
\hline $\begin{array}{l}\text { [ST (2:0)] (5Z_7E)-(3S)- } \\
\text { 9_10-seco-5_7_10(19)- } \\
\text { cholestatriene-3_25-diol }\end{array}$ & 400.3 & 7.3 & $\mathrm{C} 27 \mathrm{H} 44 \mathrm{O} 2$ & $\begin{array}{l}\text { Sterol } \\
\text { lipids }\end{array}$ & $\begin{array}{l}\text { Secosteroi } \\
\text { ds }\end{array}$ & $\begin{array}{l}19497 \\
.8\end{array}$ & $\begin{array}{l}11392 . \\
1\end{array}$ & $\begin{array}{l}16448 . \\
6\end{array}$ & $\begin{array}{l}26178 . \\
3\end{array}$ & $\begin{array}{l}15208 \\
7\end{array}$ & $\begin{array}{l}18017 . \\
5\end{array}$ & $\begin{array}{l}22387 . \\
1\end{array}$ & $\begin{array}{l}11527 . \\
4\end{array}$ & $\begin{array}{l}12155 . \\
7\end{array}$ & $\begin{array}{l}14372 . \\
0\end{array}$ & 7908.0 & $\begin{array}{l}10226 . \\
9\end{array}$ \\
\hline $\begin{array}{l}\text { [ST (20:4)] cholest-5-en- } \\
\text { 3beta-yl (15S-hydroperoxy- } \\
\text { 5Z_8Z_12E_14Z- } \\
\text { eicosatetraenoate) }\end{array}$ & 704.6 & 10.9 & $\mathrm{C} 47 \mathrm{H} 76 \mathrm{O} 4$ & $\begin{array}{l}\text { Sterol } \\
\text { lipids }\end{array}$ & na & 0.0 & 0.0 & 0.0 & 6857.8 & 0.0 & 0.0 & 0.0 & 0.0 & 0.0 & 0.0 & 0.0 & 0.0 \\
\hline $\begin{array}{l}\text { [ST hydrox] 3alpha-hydroxy- } \\
\text { 5alpha-androstan-17-one }\end{array}$ & 290.2 & 6.3 & $\mathrm{C} 19 \mathrm{H} 30 \mathrm{O} 2$ & $\begin{array}{l}\text { Sterol } \\
\text { lipids }\end{array}$ & Steroids & $\begin{array}{l}3045 . \\
3\end{array}$ & 7389.2 & $\begin{array}{l}12738 . \\
3\end{array}$ & $\begin{array}{l}17491 . \\
4\end{array}$ & $\begin{array}{l}23835 . \\
5\end{array}$ & 3463.3 & 0.0 & 0.0 & 0.0 & 0.0 & 0.0 & 0.0 \\
\hline $\begin{array}{l}\text { [ST hydrox] N-(3alpha- } \\
\text { hydroxy-5beta-cholan-24- } \\
\text { oyl)-glycine 3-sulfate }\end{array}$ & 549.3 & 1.6 & $\mathrm{C} 26 \mathrm{H} 43 \mathrm{NO} 7 \mathrm{~S}$ & $\begin{array}{l}\text { Sterol } \\
\text { lipids }\end{array}$ & $\begin{array}{l}\text { Steroid } \\
\text { conjugates }\end{array}$ & $\begin{array}{l}39929 \\
9.8\end{array}$ & $\begin{array}{l}29785 . \\
5\end{array}$ & $\begin{array}{l}378554 \\
.1\end{array}$ & $\begin{array}{l}372370 \\
.7\end{array}$ & $\begin{array}{l}422316 \\
.9\end{array}$ & $\begin{array}{l}126532 \\
.8\end{array}$ & 0.0 & 0.0 & 0.0 & 0.0 & 0.0 & 0.0 \\
\hline $\begin{array}{l}\text { [ST hydroxy(2:0/2:0)] (7E)- } \\
\text { (3S_6RS)-3-hydroxy-6_19- } \\
\text { epithio-23_24-dinor-9_10- } \\
\text { seco-5(10)_7-choladien-22-al } \\
\text { S_S-dioxide }\end{array}$ & 392.2 & 1.3 & $\mathrm{C} 22 \mathrm{H} 32 \mathrm{O} 4 \mathrm{~S}$ & $\begin{array}{l}\text { Sterol } \\
\text { lipids }\end{array}$ & $\begin{array}{l}\text { Secosteroi } \\
\text { ds }\end{array}$ & $\begin{array}{l}94590 \\
.0\end{array}$ & $\begin{array}{l}77458 . \\
5\end{array}$ & $\begin{array}{l}63832 . \\
9\end{array}$ & $\begin{array}{l}102097 \\
4.5\end{array}$ & $\begin{array}{l}175714 \\
.4\end{array}$ & $\begin{array}{l}261293 \\
.9\end{array}$ & $\begin{array}{l}257341 \\
.7\end{array}$ & $\begin{array}{l}105813 \\
.5\end{array}$ & $\begin{array}{l}106866 \\
.5\end{array}$ & $\begin{array}{l}759626 \\
.5\end{array}$ & $\begin{array}{l}29615 . \\
8\end{array}$ & $\begin{array}{l}271474 \\
.3\end{array}$ \\
\hline
\end{tabular}


[ST hydroxy(4:0)]

beta_3alpha_7alpha_12alph

-Tetrahydroxy-5beta-cholan-

24-oic Acid

5_7_10(19)-cholestatriene

1_3-di-(9Z_12Z-

octadecadienoyl)-2-hydroxyglycerol (d5)

1-(6-[5]-ladderane-hexanyl)2-(8-[3]-ladderane-octanyl)sn-glycerol

\section{1-(8-[5]-ladderane-octanoyl)-} 2-(8-[3]-ladderane-octanyl)-

sn-glycerol

1-(8-[5]-ladderane-octanyl)-2(8-[3]-ladderane-octanyl)-snglycerol

1-O-alpha-D-glucopyranosyl- $\quad 714.6$ (2-hexadecanoyloxy)-

eicosane

1-O-alpha-D-glucopyranosyl(2-tetradecanoyloxy)-eicosane

686.6

13.9

$\mathrm{C} 40 \mathrm{H} 78 \mathrm{O}$

Sterol
lipids

Glyceroli na pids

$\mathrm{C} 41 \mathrm{H} 66 \mathrm{O} 3$

Glyc

pids

Dirady

25
.6

39759
4

.

55061. 5274

741. $73483 . \quad 48224$

Glyceroli

Glycerol

Diradylg

32863

47868
0

61996

996. 61079. 42210. 56

99. $\quad 0.0$

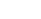

0.0

Glyceroli Diradylgly $\quad 76290 \quad 92932$

$\begin{array}{lllllllll}\text { Glyceroli } & \text { Diradylgly } & 76290 & 92932 . & 146513 & 129241 & 123877 & 129099 & 0.0\end{array}$

0.0

0.0

0.0

0.0

C42 18208

Acyls

Fatty acy

$\begin{array}{lllllll}16217 & 254282 & 375483 & 414572 & 138984 & 390107 & 0.0\end{array}$

0.0

0.0

glycosides

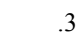

(

$+2$

$\begin{array}{lllll}\text { Fatty acyl } & 19683 & 290748 & 405164 & 45\end{array}$

$\begin{array}{lll}405164-454079 & 203862 \quad 40 \\ 9 & 0 & 0\end{array}$

732

9.9

10-Nonacosen-2-one

420.4

C29H56O

Fatty

d

hydrocarb

ons

$\begin{array}{llllllllllll}5651 . & 0.0 & 0.0 & 0.0 & 6361.9 & 0.0 & 0.0 & 0.0 & 0.0 & 0.0 & 0.0 & 0.0 \\ 9 & & & & & & & & & & & \end{array}$




\begin{tabular}{|c|c|c|c|c|c|c|c|c|c|c|c|c|c|c|c|c|c|}
\hline $\begin{array}{l}\text { 13E_15E_18Z_20Z- } \\
\text { pentacosatetraen-11-ynyl } \\
\text { acetate }\end{array}$ & 398.3 & 8.5 & $\mathrm{C} 27 \mathrm{H} 42 \mathrm{O} 2$ & $\begin{array}{l}\text { Fatty } \\
\text { Acyls }\end{array}$ & $\begin{array}{l}\text { Fatty } \\
\text { esters }\end{array}$ & 0.0 & 0.0 & 0.0 & 8344.6 & 3095.7 & 0.0 & 0.0 & 0.0 & 3035.0 & 0.0 & 0.0 & 0.0 \\
\hline $\begin{array}{l}\text { 2-C-Methyl-D-erythritol 4- } \\
\text { phosphate }\end{array}$ & 216.0 & 1.2 & $\mathrm{C} 5 \mathrm{H} 13 \mathrm{O} 7 \mathrm{P}$ & $\begin{array}{l}\text { Lipid } \\
\text { Metabolis } \\
\mathrm{m}\end{array}$ & $\begin{array}{l}\text { Biosynthes } \\
\text { is of } \\
\text { steroids }\end{array}$ & $\begin{array}{l}20706 \\
26.0\end{array}$ & $\begin{array}{l}687595 \\
6.0\end{array}$ & $\begin{array}{l}249017 \\
7.0\end{array}$ & $\begin{array}{l}487023 \\
2.0\end{array}$ & $\begin{array}{l}147190 \\
4.9\end{array}$ & $\begin{array}{l}120065 \\
00.0\end{array}$ & 0.0 & 0.0 & 0.0 & 0.0 & 0.0 & 0.0 \\
\hline 23-Hexacosen-1-ol & 397.4 & 11.8 & $\mathrm{C} 26 \mathrm{H} 52 \mathrm{O}$ & $\begin{array}{l}\text { Fatty } \\
\text { Acyls }\end{array}$ & $\begin{array}{l}\text { Fatty } \\
\text { alcohols }\end{array}$ & $\begin{array}{l}52093 \\
.5\end{array}$ & $\begin{array}{l}34609 . \\
2\end{array}$ & $\begin{array}{l}60862 . \\
2\end{array}$ & $\begin{array}{l}130924 \\
.4\end{array}$ & $\begin{array}{l}74769 . \\
2\end{array}$ & $\begin{array}{l}97647 . \\
9\end{array}$ & $\begin{array}{l}117684 \\
.7\end{array}$ & $\begin{array}{l}101379 \\
.0\end{array}$ & $\begin{array}{l}95905 . \\
7\end{array}$ & $\begin{array}{l}82715 . \\
2\end{array}$ & $\begin{array}{l}116093 \\
.4\end{array}$ & $\begin{array}{l}76590 . \\
7\end{array}$ \\
\hline 29:3(5Z_9Z_23Z) & 432.4 & 16.6 & $\mathrm{C} 29 \mathrm{H} 52 \mathrm{O} 2$ & $\begin{array}{l}\text { Fatty } \\
\text { Acyls }\end{array}$ & $\begin{array}{l}\text { Fatty } \\
\text { Acids and } \\
\text { Conjugates }\end{array}$ & $\begin{array}{l}12180 \\
.2\end{array}$ & $\begin{array}{l}19068 . \\
2\end{array}$ & 8168.9 & 8412.8 & 8016.1 & $\begin{array}{l}12887 . \\
0\end{array}$ & 3611.9 & 0.0 & 4258.1 & 0.0 & 0.0 & 0.0 \\
\hline 30:3(5Z_9Z_23Z) & 446.4 & 17.2 & $\mathrm{C} 30 \mathrm{H} 54 \mathrm{O} 2$ & $\begin{array}{l}\text { Fatty } \\
\text { Acyls }\end{array}$ & $\begin{array}{l}\text { Fatty } \\
\text { Acids and } \\
\text { Conjugates }\end{array}$ & $\begin{array}{l}7590 . \\
3\end{array}$ & 4330.8 & 4102.7 & 3314.6 & 4467.0 & 9372.6 & 0.0 & 0.0 & 0.0 & 0.0 & 0.0 & 0.0 \\
\hline $\begin{array}{l}\text { 3beta_4beta_5-Trimethoxy- } \\
\text { 4'-hydroxy-(6:7)-2_2- } \\
\text { dimethylpyranoflavan }\end{array}$ & 434.1 & 2.0 & $\mathrm{C} 23 \mathrm{H} 26 \mathrm{O} 6$ & $\begin{array}{l}\text { Fatty } \\
\text { Acyls }\end{array}$ & Flavonoids & $\begin{array}{l}31627 \\
.8\end{array}$ & $\begin{array}{l}18727 . \\
9\end{array}$ & $\begin{array}{l}23894 . \\
1\end{array}$ & $\begin{array}{l}27701 . \\
3\end{array}$ & $\begin{array}{l}21898 \\
5\end{array}$ & $\begin{array}{l}18742 . \\
6\end{array}$ & 0.0 & 0.0 & 0.0 & 0.0 & 0.0 & 0.0 \\
\hline $\begin{array}{l}\text { 3Z_6Z_9Z_12Z_15Z- } \\
\text { Tricosapentaene }\end{array}$ & 331.3 & 4.1 & $\mathrm{C} 23 \mathrm{H} 38$ & $\begin{array}{l}\text { Fatty } \\
\text { Acyls }\end{array}$ & $\begin{array}{l}\text { Hydrocarb } \\
\text { ons }\end{array}$ & $\begin{array}{l}83102 \\
.1\end{array}$ & $\begin{array}{l}81581 . \\
5\end{array}$ & $\begin{array}{l}49688 . \\
8\end{array}$ & $\begin{array}{l}248242 \\
.5\end{array}$ & $\begin{array}{l}170636 \\
6.5\end{array}$ & $\begin{array}{l}228305 \\
.8\end{array}$ & 0.0 & 0.0 & 0.0 & 0.0 & 0.0 & 0.0 \\
\hline 4Z_26Z-Hentriacontadiene & 432.5 & 12.5 & $\mathrm{C} 31 \mathrm{H} 60$ & $\begin{array}{l}\text { Fatty } \\
\text { Acyls }\end{array}$ & $\begin{array}{l}\text { Hydrocarb } \\
\text { ons }\end{array}$ & $\begin{array}{l}57810 \\
.5\end{array}$ & $\begin{array}{l}82613 . \\
5\end{array}$ & $\begin{array}{l}144290 \\
.7\end{array}$ & $\begin{array}{l}175141 \\
.4\end{array}$ & $\begin{array}{l}136356 \\
.8\end{array}$ & $\begin{array}{l}149089 \\
.7\end{array}$ & 0.0 & 0.0 & 0.0 & 0.0 & 0.0 & 0.0 \\
\hline $\begin{array}{l}\text { 5beta-cholestan- } \\
\text { 3alpha_4alpha_11beta_12beta } \\
\text { 21-pentol-3_21-disulphate }\end{array}$ & 612.3 & 10.6 & $\begin{array}{l}\mathrm{C} 27 \mathrm{H} 48 \mathrm{O} 11 \mathrm{~S} \\
2\end{array}$ & $\begin{array}{l}\text { Sterol } \\
\text { Lipids }\end{array}$ & Sterols & $\begin{array}{l}21534 \\
7.4\end{array}$ & $\begin{array}{l}105020 \\
.3\end{array}$ & $\begin{array}{l}136047 \\
.3\end{array}$ & $\begin{array}{l}112195 \\
.7\end{array}$ & $\begin{array}{l}23532 . \\
3\end{array}$ & $\begin{array}{l}186918 \\
.5\end{array}$ & $\begin{array}{l}16365 . \\
4\end{array}$ & $\begin{array}{l}14972 . \\
4\end{array}$ & $\begin{array}{l}41285 . \\
3\end{array}$ & $\begin{array}{l}15801 . \\
8\end{array}$ & $\begin{array}{l}14630 . \\
5\end{array}$ & $\begin{array}{l}14378 . \\
5\end{array}$ \\
\hline 9Z_19Z-Pentatriacontadiene & 488.5 & 16.2 & $\mathrm{C} 35 \mathrm{H} 68$ & $\begin{array}{l}\text { Fatty } \\
\text { Acyls }\end{array}$ & $\begin{array}{l}\text { Hydrocarb } \\
\text { ons }\end{array}$ & $\begin{array}{l}23420 \\
.9\end{array}$ & $\begin{array}{l}37630 . \\
6\end{array}$ & $\begin{array}{l}47080 . \\
1\end{array}$ & $\begin{array}{l}68006 . \\
6\end{array}$ & $\begin{array}{l}14612 . \\
5\end{array}$ & $\begin{array}{l}64448 . \\
9\end{array}$ & 0.0 & 0.0 & 0.0 & 0.0 & 0.0 & 0.0 \\
\hline Alatanin 2 & 831.2 & 17.2 & $\mathrm{C} 39 \mathrm{H} 43 \mathrm{O} 20$ & $\begin{array}{l}\text { Polyketid } \\
\text { es }\end{array}$ & Flavonoids & 0.0 & 0.0 & 0.0 & 0.0 & 0.0 & 0.0 & $\begin{array}{l}120428 \\
79.0\end{array}$ & $\begin{array}{l}117151 \\
67.0\end{array}$ & $\begin{array}{l}903088 \\
8.0\end{array}$ & $\begin{array}{l}836890 \\
4.0\end{array}$ & $\begin{array}{l}131621 \\
43.0\end{array}$ & $\begin{array}{l}881374 \\
3.0\end{array}$ \\
\hline Anandamide & 383.3 & 5.5 & $\mathrm{C} 22 \mathrm{H} 37 \mathrm{NO} 2$ & $\begin{array}{l}\text { Fatty } \\
\text { Acyls }\end{array}$ & $\begin{array}{l}\text { anandamid } \\
\mathrm{e} \\
\text { degradatio } \\
\mathrm{n}\end{array}$ & $\begin{array}{l}3295 . \\
1\end{array}$ & 3544.7 & $\begin{array}{l}13661 . \\
5\end{array}$ & $\begin{array}{l}16707 . \\
8\end{array}$ & $\begin{array}{l}29992 . \\
3\end{array}$ & 8143.9 & 0.0 & 0.0 & 0.0 & 0.0 & 0.0 & 0.0 \\
\hline
\end{tabular}




\begin{tabular}{|c|c|c|c|c|c|c|c|c|c|c|c|c|c|c|c|c|c|}
\hline CDP-choline & 488.1 & 1.2 & $\begin{array}{l}\mathrm{C} 14 \mathrm{H} 26 \mathrm{~N} 4 \mathrm{O} 1 \\
1 \mathrm{P} 2\end{array}$ & $\begin{array}{l}\text { Lipid } \\
\text { Metabolis } \\
\mathrm{m}\end{array}$ & $\begin{array}{l}\text { Glyceroph } \\
\text { ospholipid } \\
\text { metabolis } \\
\text { m }\end{array}$ & $\begin{array}{l}24978 \\
1.5\end{array}$ & $\begin{array}{l}374155 \\
.9\end{array}$ & 0.0 & 0.0 & $\begin{array}{l}31367 . \\
2\end{array}$ & $\begin{array}{l}123179 \\
6.9\end{array}$ & 0.0 & 0.0 & 0.0 & 0.0 & 0.0 & 0.0 \\
\hline $\operatorname{Cer}(\mathrm{d}-\mathrm{h}$ 35:0) & 569.5 & 18.5 & $\mathrm{C} 35 \mathrm{H} 71 \mathrm{NO} 4$ & $\begin{array}{l}\text { Sphingoli } \\
\text { pids }\end{array}$ & Ceramides & $\begin{array}{l}46786 \\
.8\end{array}$ & $\begin{array}{l}72843 . \\
2\end{array}$ & $\begin{array}{l}34303 . \\
4\end{array}$ & $\begin{array}{l}37086 . \\
6\end{array}$ & $\begin{array}{l}14964 . \\
3\end{array}$ & $\begin{array}{l}80079 . \\
9\end{array}$ & $\begin{array}{l}12724 . \\
5\end{array}$ & $\begin{array}{l}14351 . \\
9\end{array}$ & $\begin{array}{l}13144 . \\
4\end{array}$ & $\begin{array}{l}12259 . \\
5\end{array}$ & $\begin{array}{l}14615 . \\
2\end{array}$ & $\begin{array}{l}12493 . \\
7\end{array}$ \\
\hline Cer(d39:1) & 653.6 & 15.6 & $\mathrm{C} 39 \mathrm{H} 77 \mathrm{NO} 3$ & $\begin{array}{l}\text { Sphingoli } \\
\text { pids }\end{array}$ & Ceramides & $\begin{array}{l}11868 \\
8.5\end{array}$ & $\begin{array}{l}185545 \\
.3\end{array}$ & $\begin{array}{l}257073 \\
.4\end{array}$ & $\begin{array}{l}318045 \\
.3\end{array}$ & $\begin{array}{l}157893 \\
.8\end{array}$ & $\begin{array}{l}259638 \\
.8\end{array}$ & 0.0 & 0.0 & 0.0 & 0.0 & 0.0 & 0.0 \\
\hline Choline phosphate & 183.1 & 1.5 & $\mathrm{C} 5 \mathrm{H} 14 \mathrm{NO} 4 \mathrm{P}$ & $\begin{array}{l}\text { Lipid } \\
\text { Metabolis } \\
\mathrm{m}\end{array}$ & $\begin{array}{l}\text { Glyceroph } \\
\text { ospholipid } \\
\text { metabolis } \\
\mathrm{m} \text { _Glycin } \\
\mathrm{e} \text {, serine } \\
\text { and } \\
\text { threonine } \\
\text { metabolis } \\
\mathrm{m}\end{array}$ & 0.0 & 0.0 & 0.0 & 0.0 & 0.0 & 0.0 & $\begin{array}{l}26099 . \\
5\end{array}$ & $\begin{array}{l}46312 . \\
0\end{array}$ & $\begin{array}{l}24060 . \\
9\end{array}$ & $\begin{array}{l}25232 . \\
7\end{array}$ & $\begin{array}{l}17562 . \\
8\end{array}$ & $\begin{array}{l}21931 . \\
2\end{array}$ \\
\hline $\begin{array}{l}\text { Cyanidin 3-(6"- } \\
\text { sinapylsophoroside)-5- } \\
\text { glucoside }\end{array}$ & 979.3 & 19.9 & $\mathrm{C} 44 \mathrm{H} 51 \mathrm{O} 25$ & $\begin{array}{l}\text { Polyketid } \\
\text { es }\end{array}$ & Flavonoids & 0.0 & 0.0 & 0.0 & 0.0 & 0.0 & 0.0 & $\begin{array}{l}451991 \\
0.5\end{array}$ & $\begin{array}{l}509537 \\
5.0\end{array}$ & $\begin{array}{l}303174 \\
6.0\end{array}$ & $\begin{array}{l}316556 \\
5.5\end{array}$ & $\begin{array}{l}339194 \\
5.8\end{array}$ & $\begin{array}{l}307034 \\
2.8\end{array}$ \\
\hline $\operatorname{DG}(31: 3)$ & 548.4 & 12.8 & $\mathrm{C} 34 \mathrm{H} 60 \mathrm{O} 5$ & $\begin{array}{l}\text { Glyceroli } \\
\text { pids }\end{array}$ & $\begin{array}{l}\text { Diradylgly } \\
\text { cerols }\end{array}$ & $\begin{array}{l}10350 \\
.4\end{array}$ & $\begin{array}{l}15332 . \\
1\end{array}$ & 0.0 & $\begin{array}{l}19496 . \\
0\end{array}$ & $\begin{array}{l}14405 . \\
6\end{array}$ & $\begin{array}{l}17383 . \\
0\end{array}$ & 5662.4 & 3738.6 & 6584.7 & 7040.1 & $\begin{array}{l}10671 . \\
3\end{array}$ & 7209.6 \\
\hline DG(35:4) & 602.5 & 14.3 & $\mathrm{C} 38 \mathrm{H} 66 \mathrm{O} 5$ & $\begin{array}{l}\text { Glyceroli } \\
\text { pids }\end{array}$ & $\begin{array}{l}\text { Diradylgly } \\
\text { cerols }\end{array}$ & $\begin{array}{l}87643 \\
.9\end{array}$ & $\begin{array}{l}155261 \\
.6\end{array}$ & $\begin{array}{l}52053 . \\
3\end{array}$ & $\begin{array}{l}113628 \\
.4\end{array}$ & $\begin{array}{l}88396 . \\
4\end{array}$ & $\begin{array}{l}85148 . \\
1\end{array}$ & 0.0 & 0.0 & 0.0 & 0.0 & 0.0 & 0.0 \\
\hline DG(36:4) & 616.5 & 15.1 & $\mathrm{C} 39 \mathrm{H} 68 \mathrm{O} 5$ & $\begin{array}{l}\text { Glyceroli } \\
\text { pids }\end{array}$ & $\begin{array}{l}\text { Diradylgly } \\
\text { cerols }\end{array}$ & $\begin{array}{l}48648 \\
.4\end{array}$ & $\begin{array}{l}79332 . \\
1\end{array}$ & $\begin{array}{l}68952 . \\
2\end{array}$ & $\begin{array}{l}98005 . \\
6\end{array}$ & $\begin{array}{l}63487 . \\
3\end{array}$ & $\begin{array}{l}62670 . \\
3\end{array}$ & 0.0 & 0.0 & 0.0 & 0.0 & 0.0 & 0.0 \\
\hline $\operatorname{DG}(37: 4)$ & 630.5 & 15.7 & $\mathrm{C} 40 \mathrm{H} 70 \mathrm{O} 5$ & $\begin{array}{l}\text { Glyceroli } \\
\text { pids }\end{array}$ & $\begin{array}{l}\text { Diradylgly } \\
\text { cerols }\end{array}$ & $\begin{array}{l}52012 \\
.8\end{array}$ & $\begin{array}{l}89474 . \\
9\end{array}$ & $\begin{array}{l}81292 . \\
2\end{array}$ & $\begin{array}{l}105708 \\
.6\end{array}$ & $\begin{array}{l}51428 . \\
4\end{array}$ & $\begin{array}{l}67200 . \\
2\end{array}$ & 0.0 & 0.0 & 0.0 & 0.0 & 0.0 & 0.0 \\
\hline $\operatorname{DG}(37: 5)$ & 628.5 & 14.9 & $\mathrm{C} 40 \mathrm{H} 68 \mathrm{O} 5$ & $\begin{array}{l}\text { Glyceroli } \\
\text { pids }\end{array}$ & $\begin{array}{l}\text { Diradylgly } \\
\text { cerols }\end{array}$ & $\begin{array}{l}92430 \\
.6\end{array}$ & $\begin{array}{l}128100 \\
.1\end{array}$ & $\begin{array}{l}127914 \\
.6\end{array}$ & $\begin{array}{l}212252 \\
.3\end{array}$ & $\begin{array}{l}86646 . \\
2\end{array}$ & $\begin{array}{l}98981 . \\
7\end{array}$ & 0.0 & 0.0 & 3291.9 & 3275.7 & 0.0 & 0.0 \\
\hline $\operatorname{DG}(37: 6)$ & 626.5 & 14.0 & $\mathrm{C} 40 \mathrm{H} 66 \mathrm{O} 5$ & $\begin{array}{l}\text { Glyceroli } \\
\text { pids }\end{array}$ & $\begin{array}{l}\text { Diradylgly } \\
\text { cerols }\end{array}$ & $\begin{array}{l}17116 \\
0.7\end{array}$ & $\begin{array}{l}249948 \\
.8\end{array}$ & $\begin{array}{l}191357 \\
.8\end{array}$ & $\begin{array}{l}209937 \\
.3\end{array}$ & $\begin{array}{l}106655 \\
.0\end{array}$ & $\begin{array}{l}137899 \\
.8\end{array}$ & 0.0 & 0.0 & 0.0 & 0.0 & 0.0 & 0.0 \\
\hline $\operatorname{DG}(38: 2)$ & 648.6 & 13.7 & $\mathrm{C} 41 \mathrm{H} 76 \mathrm{O} 5$ & $\begin{array}{l}\text { Glyceroli } \\
\text { pids }\end{array}$ & $\begin{array}{l}\text { Diradylgly } \\
\text { cerols }\end{array}$ & $\begin{array}{l}70133 \\
.0\end{array}$ & $\begin{array}{l}85598 . \\
7\end{array}$ & $\begin{array}{l}93581 . \\
8\end{array}$ & $\begin{array}{l}99368 . \\
9\end{array}$ & $\begin{array}{l}116501 \\
.0\end{array}$ & $\begin{array}{l}114195 \\
.8\end{array}$ & 0.0 & 0.0 & 0.0 & 0.0 & 0.0 & 0.0 \\
\hline $\operatorname{DG}(38: 4)$ & 661.6 & 15.6 & $\mathrm{C} 41 \mathrm{H} 72 \mathrm{O} 5$ & $\begin{array}{l}\text { Glyceroli } \\
\text { pids }\end{array}$ & $\begin{array}{l}\text { Diradylgly } \\
\text { cerols }\end{array}$ & $\begin{array}{l}77525 \\
.5\end{array}$ & $\begin{array}{l}120045 \\
.8\end{array}$ & $\begin{array}{l}153842 \\
.4\end{array}$ & $\begin{array}{l}213878 \\
.4\end{array}$ & $\begin{array}{l}64599 . \\
0\end{array}$ & $\begin{array}{l}77446 . \\
5\end{array}$ & 0.0 & 0.0 & 0.0 & 0.0 & 0.0 & 0.0 \\
\hline
\end{tabular}




\begin{tabular}{|c|c|c|c|c|c|c|c|c|c|c|c|c|c|c|c|c|c|}
\hline DG(38:5) & 659.5 & 14.8 & $\mathrm{C} 41 \mathrm{H} 70 \mathrm{O} 5$ & $\begin{array}{l}\text { Glyceroli } \\
\text { pids }\end{array}$ & $\begin{array}{l}\text { Diradylgly } \\
\text { cerols }\end{array}$ & $\begin{array}{l}12608 \\
1.2\end{array}$ & $\begin{array}{l}186788 \\
.7\end{array}$ & $\begin{array}{l}201174 \\
.9\end{array}$ & $\begin{array}{l}216003 \\
.3\end{array}$ & $\begin{array}{l}105760 \\
.9\end{array}$ & $\begin{array}{l}120911 \\
.6\end{array}$ & 0.0 & 0.0 & 0.0 & 0.0 & 0.0 & 0.0 \\
\hline DG(38:6) & 640.5 & 14.7 & C41H68O5 & $\begin{array}{l}\text { Glyceroli } \\
\text { pids }\end{array}$ & $\begin{array}{l}\text { Diradylgly } \\
\text { cerols }\end{array}$ & $\begin{array}{l}15271 \\
8.2\end{array}$ & $\begin{array}{l}234633 \\
.5\end{array}$ & $\begin{array}{l}229240 \\
.1\end{array}$ & $\begin{array}{l}287593 \\
.3\end{array}$ & $\begin{array}{l}126670 \\
.9\end{array}$ & $\begin{array}{l}168874 \\
.0\end{array}$ & 0.0 & 0.0 & 0.0 & 0.0 & 0.0 & 0.0 \\
\hline DG(39:5) & 656.5 & 16.2 & $\mathrm{C} 42 \mathrm{H} 72 \mathrm{O} 5$ & $\begin{array}{l}\text { Glyceroli } \\
\text { pids }\end{array}$ & $\begin{array}{l}\text { Diradylgly } \\
\text { cerols }\end{array}$ & $\begin{array}{l}28677 \\
.1\end{array}$ & $\begin{array}{l}37531 . \\
8\end{array}$ & $\begin{array}{l}42227 . \\
2\end{array}$ & $\begin{array}{l}69697 . \\
9\end{array}$ & $\begin{array}{l}35458 . \\
8\end{array}$ & $\begin{array}{l}29133 . \\
6\end{array}$ & 4790.4 & 4502.4 & 9162.4 & 3792.4 & 0.0 & 8566.9 \\
\hline DG(39:6) & 654.5 & 15.4 & $\mathrm{C} 42 \mathrm{H} 70 \mathrm{O} 5$ & $\begin{array}{l}\text { Glyceroli } \\
\text { pids }\end{array}$ & $\begin{array}{l}\text { Diradylgly } \\
\text { cerols }\end{array}$ & $\begin{array}{l}11775 \\
3.5\end{array}$ & $\begin{array}{l}222114 \\
.2\end{array}$ & $\begin{array}{l}202131 \\
.3\end{array}$ & $\begin{array}{l}249250 \\
.5\end{array}$ & $\begin{array}{l}51120 . \\
8\end{array}$ & $\begin{array}{l}151874 \\
.0\end{array}$ & 0.0 & 0.0 & 0.0 & 0.0 & 0.0 & 0.0 \\
\hline DG(39:7) & 652.5 & 14.9 & $\mathrm{C} 42 \mathrm{H} 68 \mathrm{O} 5$ & $\begin{array}{l}\text { Glyceroli } \\
\text { pids }\end{array}$ & $\begin{array}{l}\text { Diradylgly } \\
\text { cerols }\end{array}$ & $\begin{array}{l}26226 \\
4.7\end{array}$ & $\begin{array}{l}496650 \\
.2\end{array}$ & $\begin{array}{l}517582 \\
.0\end{array}$ & $\begin{array}{l}616103 \\
.6\end{array}$ & $\begin{array}{l}107772 \\
.7\end{array}$ & $\begin{array}{l}449280 \\
.8\end{array}$ & 0.0 & 0.0 & 0.0 & 0.0 & 0.0 & 0.0 \\
\hline DG(40:10) & 677.5 & 12.4 & $\mathrm{C} 43 \mathrm{H} 64 \mathrm{O} 5$ & $\begin{array}{l}\text { Glyceroli } \\
\text { pids }\end{array}$ & $\begin{array}{l}\text { Diradylgly } \\
\text { cerols }\end{array}$ & $\begin{array}{l}26774 \\
.0\end{array}$ & $\begin{array}{l}10970 . \\
4\end{array}$ & $\begin{array}{l}10556 . \\
8\end{array}$ & $\begin{array}{l}18693 . \\
9\end{array}$ & $\begin{array}{l}15661 . \\
7\end{array}$ & $\begin{array}{l}20878 . \\
6\end{array}$ & 0.0 & 0.0 & 0.0 & 0.0 & 0.0 & 0.0 \\
\hline DG(40:6) & 668.5 & 16.2 & $\mathrm{C} 43 \mathrm{H} 72 \mathrm{O} 5$ & $\begin{array}{l}\text { Glyceroli } \\
\text { pids }\end{array}$ & $\begin{array}{l}\text { Diradylgly } \\
\text { cerols }\end{array}$ & $\begin{array}{l}16014 \\
7.7\end{array}$ & $\begin{array}{l}272820 \\
.9\end{array}$ & $\begin{array}{l}352358 \\
.2\end{array}$ & $\begin{array}{l}476587 \\
.2\end{array}$ & $\begin{array}{l}81119 . \\
3\end{array}$ & $\begin{array}{l}220265 \\
.0\end{array}$ & 0.0 & 0.0 & 0.0 & 0.0 & 0.0 & 0.0 \\
\hline DG(40:7) & 683.5 & 14.4 & $\mathrm{C} 43 \mathrm{H} 70 \mathrm{O} 5$ & $\begin{array}{l}\text { Glyceroli } \\
\text { pids }\end{array}$ & $\begin{array}{l}\text { Diradylgly } \\
\text { cerols }\end{array}$ & $\begin{array}{l}50523 \\
1.6\end{array}$ & $\begin{array}{l}743877 \\
.6\end{array}$ & $\begin{array}{l}872149 \\
.6\end{array}$ & $\begin{array}{l}104961 \\
9.5\end{array}$ & $\begin{array}{l}142462 \\
.1\end{array}$ & $\begin{array}{l}698334 \\
.5\end{array}$ & 0.0 & 0.0 & 0.0 & 0.0 & 0.0 & 0.0 \\
\hline DG(41:7) & 680.5 & 16.0 & $\mathrm{C} 44 \mathrm{H} 72 \mathrm{O} 5$ & $\begin{array}{l}\text { Glyceroli } \\
\text { pids }\end{array}$ & $\begin{array}{l}\text { Diradylgly } \\
\text { cerols }\end{array}$ & $\begin{array}{l}36572 \\
.3\end{array}$ & $\begin{array}{l}61458 . \\
4\end{array}$ & $\begin{array}{l}70946 . \\
3\end{array}$ & $\begin{array}{l}103085 \\
.7\end{array}$ & $\begin{array}{l}28278 . \\
6\end{array}$ & $\begin{array}{l}45755 . \\
5\end{array}$ & 0.0 & 0.0 & 0.0 & 0.0 & 0.0 & 0.0 \\
\hline $\mathrm{DG}(42: 2)$ & 704.6 & 16.5 & $\mathrm{C} 45 \mathrm{H} 84 \mathrm{O} 5$ & $\begin{array}{l}\text { Glyceroli } \\
\text { pids }\end{array}$ & $\begin{array}{l}\text { Diradylgly } \\
\text { cerols }\end{array}$ & $\begin{array}{l}31435 \\
.2\end{array}$ & $\begin{array}{l}51031 . \\
2\end{array}$ & $\begin{array}{l}64566 . \\
1\end{array}$ & $\begin{array}{l}84772 . \\
1\end{array}$ & $\begin{array}{l}37224 . \\
6\end{array}$ & $\begin{array}{l}75762 . \\
9\end{array}$ & 0.0 & 0.0 & 0.0 & 0.0 & 0.0 & 0.0 \\
\hline DG(42:9) & 690.5 & 18.5 & $\mathrm{C} 45 \mathrm{H} 70 \mathrm{O} 5$ & $\begin{array}{l}\text { Glyceroli } \\
\text { pids }\end{array}$ & $\begin{array}{l}\text { Diradylgly } \\
\text { cerols }\end{array}$ & $\begin{array}{l}3862 . \\
0\end{array}$ & 7853.7 & 0.0 & $\begin{array}{l}12975 . \\
2\end{array}$ & 8177.8 & 0.0 & 0.0 & 0.0 & 0.0 & 0.0 & 0.0 & 0.0 \\
\hline DG(44:0) & 736.7 & 1.5 & $\mathrm{C} 47 \mathrm{H} 92 \mathrm{O} 5$ & $\begin{array}{l}\text { Glyceroli } \\
\text { pids }\end{array}$ & $\begin{array}{l}\text { Diradylgly } \\
\text { cerols }\end{array}$ & 0.0 & 0.0 & 0.0 & 0.0 & 0.0 & 0.0 & $\begin{array}{l}137985 \\
3.5\end{array}$ & $\begin{array}{l}104515 \\
3.6\end{array}$ & $\begin{array}{l}886913 \\
.2\end{array}$ & $\begin{array}{l}918977 \\
.9\end{array}$ & $\begin{array}{l}591527 \\
.9\end{array}$ & $\begin{array}{l}621476 \\
.4\end{array}$ \\
\hline DG(44:2) & 732.7 & 18.0 & $\mathrm{C} 47 \mathrm{H} 88 \mathrm{O} 5$ & $\begin{array}{l}\text { Glyceroli } \\
\text { pids }\end{array}$ & $\begin{array}{l}\text { Diradylgly } \\
\text { cerols }\end{array}$ & $\begin{array}{l}21108 \\
.0\end{array}$ & $\begin{array}{l}25013 . \\
0\end{array}$ & $\begin{array}{l}31523 . \\
7\end{array}$ & $\begin{array}{l}35793 . \\
4\end{array}$ & $\begin{array}{l}18153 . \\
1\end{array}$ & $\begin{array}{l}39444 . \\
6\end{array}$ & 0.0 & 0.0 & 0.0 & 0.0 & 0.0 & 0.0 \\
\hline DG(P-32:1) & 567.5 & 12.0 & $\mathrm{C} 35 \mathrm{H} 66 \mathrm{O} 4$ & $\begin{array}{l}\text { Glyceroli } \\
\text { pids }\end{array}$ & $\begin{array}{l}\text { Diradylgly } \\
\text { cerols }\end{array}$ & $\begin{array}{l}83140 \\
.9\end{array}$ & $\begin{array}{l}112277 \\
.7\end{array}$ & $\begin{array}{l}127448 \\
.7\end{array}$ & $\begin{array}{l}140757 \\
.4\end{array}$ & $\begin{array}{l}143245 \\
.3\end{array}$ & $\begin{array}{l}145283 \\
.3\end{array}$ & $\begin{array}{l}97094 . \\
7\end{array}$ & $\begin{array}{l}100377 \\
.7\end{array}$ & $\begin{array}{l}97653 . \\
2\end{array}$ & $\begin{array}{l}91480 . \\
8\end{array}$ & $\begin{array}{l}96703 . \\
8\end{array}$ & $\begin{array}{l}103731 \\
.1\end{array}$ \\
\hline $\begin{array}{l}\text { Dihydroxy-oxo-prostadienoic } \\
\text { acid }\end{array}$ & 378.2 & 5.7 & $\mathrm{C} 20 \mathrm{H} 28 \mathrm{D} 4 \mathrm{O} 5$ & $\begin{array}{l}\text { Fatty } \\
\text { Acyls }\end{array}$ & na & 0.0 & 0.0 & 0.0 & 0.0 & 0.0 & 0.0 & $\begin{array}{l}599690 \\
.4\end{array}$ & $\begin{array}{l}527233 \\
.3\end{array}$ & $\begin{array}{l}348234 \\
.4\end{array}$ & $\begin{array}{l}355987 \\
.8\end{array}$ & $\begin{array}{l}488763 \\
.7\end{array}$ & $\begin{array}{l}514327 \\
.0\end{array}$ \\
\hline Docosahexaenoicacid & 328.2 & 6.9 & $\mathrm{C} 22 \mathrm{H} 32 \mathrm{O} 2$ & $\begin{array}{l}\text { Fatty } \\
\text { Acyls }\end{array}$ & $\begin{array}{l}\text { Biosynthes } \\
\text { is of } \\
\text { unsaturate } \\
\mathrm{d} \text { fatty } \\
\text { acids }\end{array}$ & $\begin{array}{l}12124 \\
.9\end{array}$ & $\begin{array}{l}23068 . \\
9\end{array}$ & $\begin{array}{l}43434 . \\
6\end{array}$ & $\begin{array}{l}49698 \\
1\end{array}$ & $\begin{array}{l}84549 . \\
1\end{array}$ & $\begin{array}{l}14667 . \\
7\end{array}$ & 0.0 & 0.0 & 0.0 & 0.0 & 0.0 & 0.0 \\
\hline
\end{tabular}




\begin{tabular}{|c|c|c|c|c|c|c|c|c|c|c|c|c|c|c|c|c|c|}
\hline Docosanoic acid & 340.3 & 11.4 & $\mathrm{C} 22 \mathrm{H} 44 \mathrm{O} 2$ & $\begin{array}{l}\text { Lipid } \\
\text { Metabolis } \\
\mathrm{m}\end{array}$ & $\begin{array}{l}\text { Biosynthes } \\
\text { is of } \\
\text { unsaturate } \\
\mathrm{d} \text { fatty } \\
\text { acids }\end{array}$ & $\begin{array}{l}97328 \\
.0\end{array}$ & $\begin{array}{l}85005 \\
7\end{array}$ & $\begin{array}{l}119422 \\
.3\end{array}$ & $\begin{array}{l}208721 \\
.3\end{array}$ & $\begin{array}{l}263223 \\
.0\end{array}$ & $\begin{array}{l}157317 \\
.2\end{array}$ & $\begin{array}{l}49012 . \\
7\end{array}$ & $\begin{array}{l}39605 . \\
5\end{array}$ & $\begin{array}{l}37730 \\
4\end{array}$ & $\begin{array}{l}51089 . \\
5\end{array}$ & $\begin{array}{l}59059 . \\
0\end{array}$ & $\begin{array}{l}35142 . \\
9\end{array}$ \\
\hline Dodecanoic acid & 200.2 & 10.9 & $\mathrm{C} 12 \mathrm{H} 24 \mathrm{O} 2$ & $\begin{array}{l}\text { Lipid } \\
\text { Metabolis } \\
\mathrm{m}\end{array}$ & $\begin{array}{l}\text { Fatty acid } \\
\text { biosynthes } \\
\text { is }\end{array}$ & 0.0 & 0.0 & 0.0 & 0.0 & 0.0 & 0.0 & $\begin{array}{l}132921 \\
.7\end{array}$ & $\begin{array}{l}126306 \\
.8\end{array}$ & $\begin{array}{l}128887 \\
.3\end{array}$ & $\begin{array}{l}90557 . \\
8\end{array}$ & $\begin{array}{l}109451 \\
.9\end{array}$ & $\begin{array}{l}88012 . \\
1\end{array}$ \\
\hline FA (13:2) tridecadienoic acid & 227.2 & 1.8 & $\mathrm{C} 13 \mathrm{H} 22 \mathrm{O} 2$ & $\begin{array}{l}\text { Fatty } \\
\text { Acyls }\end{array}$ & $\begin{array}{l}\text { Fatty } \\
\text { Acids and } \\
\text { Conjugates }\end{array}$ & 0.0 & 0.0 & 0.0 & 0.0 & 0.0 & 0.0 & $\begin{array}{l}309688 \\
.3\end{array}$ & $\begin{array}{l}215758 \\
.6\end{array}$ & $\begin{array}{l}275344 \\
.0\end{array}$ & $\begin{array}{l}188872 \\
.7\end{array}$ & $\begin{array}{l}603472 \\
.3\end{array}$ & $\begin{array}{l}162716 \\
.1\end{array}$ \\
\hline FA $(17: 0)$ & 270.3 & 7.9 & $\mathrm{C} 17 \mathrm{H} 34 \mathrm{O} 2$ & $\begin{array}{l}\text { Fatty } \\
\text { Acyls }\end{array}$ & $\begin{array}{l}\text { Fatty } \\
\text { Acids and } \\
\text { Conjugates }\end{array}$ & $\begin{array}{l}10684 \\
8.0\end{array}$ & $\begin{array}{l}129449 \\
.3\end{array}$ & $\begin{array}{l}183561 \\
.5\end{array}$ & $\begin{array}{l}232542 \\
.7\end{array}$ & $\begin{array}{l}273716 \\
.2\end{array}$ & $\begin{array}{l}185974 \\
.4\end{array}$ & $\begin{array}{l}51317 . \\
9\end{array}$ & $\begin{array}{l}50953 . \\
0\end{array}$ & $\begin{array}{l}40485 . \\
0\end{array}$ & $\begin{array}{l}47214 . \\
3\end{array}$ & $\begin{array}{l}86179 . \\
6\end{array}$ & $\begin{array}{l}37903 . \\
2\end{array}$ \\
\hline FA $(18: 1)$ & 282.3 & 7.6 & $\mathrm{C} 18 \mathrm{H} 34 \mathrm{O} 2$ & $\begin{array}{l}\text { Fatty } \\
\text { Acyls }\end{array}$ & $\begin{array}{l}\text { Fatty } \\
\text { Acids and } \\
\text { Conjugates }\end{array}$ & $\begin{array}{l}54390 \\
2.7\end{array}$ & $\begin{array}{l}602751 \\
.2\end{array}$ & $\begin{array}{l}965572 \\
.9\end{array}$ & $\begin{array}{l}142413 \\
0.4\end{array}$ & $\begin{array}{l}205883 \\
5.9\end{array}$ & $\begin{array}{l}863440 \\
.0\end{array}$ & 0.0 & 0.0 & 0.0 & 0.0 & 0.0 & 0.0 \\
\hline FA $(18: 2)$ & 280.2 & 6.9 & $\mathrm{C} 18 \mathrm{H} 32 \mathrm{O} 2$ & $\begin{array}{l}\text { Fatty } \\
\text { Acyls }\end{array}$ & $\begin{array}{l}\text { Fatty } \\
\text { Acids and } \\
\text { Conjugates }\end{array}$ & $\begin{array}{l}34057 \\
2.0\end{array}$ & $\begin{array}{l}270125 \\
.8\end{array}$ & $\begin{array}{l}442570 \\
.9\end{array}$ & $\begin{array}{l}772348 \\
.1\end{array}$ & $\begin{array}{l}913142 \\
.5\end{array}$ & $\begin{array}{l}425248 \\
.9\end{array}$ & 0.0 & 0.0 & 0.0 & 0.0 & 0.0 & 0.0 \\
\hline FA (18:3) & 278.2 & 6.2 & $\mathrm{C} 18 \mathrm{H} 30 \mathrm{O} 2$ & $\begin{array}{l}\text { Fatty } \\
\text { Acyls }\end{array}$ & $\begin{array}{l}\text { Fatty } \\
\text { Acids and } \\
\text { Conjugates }\end{array}$ & $\begin{array}{l}23715 \\
.2\end{array}$ & $\begin{array}{l}24031 \\
1\end{array}$ & $\begin{array}{l}38338 \\
6\end{array}$ & $\begin{array}{l}63013 . \\
5\end{array}$ & $\begin{array}{l}96212 . \\
8\end{array}$ & $\begin{array}{l}31922 . \\
1\end{array}$ & 0.0 & 0.0 & 0.0 & 0.0 & 0.0 & 0.0 \\
\hline FA (18:4) & 276.2 & 5.5 & $\mathrm{C} 18 \mathrm{H} 28 \mathrm{O} 2$ & $\begin{array}{l}\text { Fatty } \\
\text { Acyls }\end{array}$ & $\begin{array}{l}\text { Fatty } \\
\text { Acids and } \\
\text { Conjugates }\end{array}$ & 0.0 & 0.0 & 2288.6 & 3642.1 & 5686.8 & 0.0 & 0.0 & 0.0 & 0.0 & 0.0 & 0.0 & 0.0 \\
\hline FA $(19: 1)$ & 296.3 & 8.4 & $\mathrm{C} 19 \mathrm{H} 36 \mathrm{O} 2$ & $\begin{array}{l}\text { Fatty } \\
\text { Acyls }\end{array}$ & $\begin{array}{l}\text { Fatty } \\
\text { Acids and } \\
\text { Conjugates }\end{array}$ & $\begin{array}{l}61407 \\
.0\end{array}$ & $\begin{array}{l}79443 . \\
7\end{array}$ & $\begin{array}{l}194437 \\
.3\end{array}$ & $\begin{array}{l}313324 \\
.1\end{array}$ & $\begin{array}{l}727615 \\
.9\end{array}$ & $\begin{array}{l}89974 . \\
1\end{array}$ & 3789.4 & 3258.1 & 3316.0 & 5709.7 & 4566.8 & 3180.4 \\
\hline FA $(20: 0)$ & 312.3 & 10.1 & $\mathrm{C} 20 \mathrm{H} 40 \mathrm{O} 2$ & $\begin{array}{l}\text { Fatty } \\
\text { Acyls }\end{array}$ & $\begin{array}{l}\text { Fatty } \\
\text { Acids and } \\
\text { Conjugates }\end{array}$ & $\begin{array}{l}17372 \\
4.5\end{array}$ & $\begin{array}{l}124276 \\
.0\end{array}$ & $\begin{array}{l}235373 \\
.0\end{array}$ & $\begin{array}{l}355665 \\
.7\end{array}$ & $\begin{array}{l}496447 \\
.2\end{array}$ & $\begin{array}{l}275434 \\
.6\end{array}$ & $\begin{array}{l}162899 \\
.0\end{array}$ & $\begin{array}{l}151256 \\
.6\end{array}$ & $\begin{array}{l}122178 \\
.7\end{array}$ & $\begin{array}{l}134598 \\
.5\end{array}$ & $\begin{array}{l}253899 \\
.4\end{array}$ & $\begin{array}{l}109030 \\
.0\end{array}$ \\
\hline FA $(20: 1)$ & 310.3 & 8.9 & $\mathrm{C} 20 \mathrm{H} 38 \mathrm{O} 2$ & $\begin{array}{l}\text { Fatty } \\
\text { Acyls }\end{array}$ & $\begin{array}{l}\text { Fatty } \\
\text { Acids and } \\
\text { Conjugates }\end{array}$ & $\begin{array}{l}32879 \\
.8\end{array}$ & $\begin{array}{l}33164 . \\
0\end{array}$ & $\begin{array}{l}52007 . \\
2\end{array}$ & $\begin{array}{l}65545 . \\
7\end{array}$ & $\begin{array}{l}161602 \\
.8\end{array}$ & $\begin{array}{l}79496 . \\
8\end{array}$ & 0.0 & 0.0 & 0.0 & 0.0 & 0.0 & 0.0 \\
\hline FA $(20: 2)$ & 308.3 & 8.2 & $\mathrm{C} 20 \mathrm{H} 36 \mathrm{O} 2$ & $\begin{array}{l}\text { Fatty } \\
\text { Acyls }\end{array}$ & $\begin{array}{l}\text { Fatty } \\
\text { Acids and } \\
\text { Conjugates }\end{array}$ & $\begin{array}{l}49637 \\
.1\end{array}$ & $\begin{array}{l}72167 \\
3\end{array}$ & $\begin{array}{l}98045 . \\
0\end{array}$ & $\begin{array}{l}113505 \\
.9\end{array}$ & $\begin{array}{l}173336 \\
.7\end{array}$ & $\begin{array}{l}76614 . \\
3\end{array}$ & 0.0 & 0.0 & 0.0 & 0.0 & 0.0 & 0.0 \\
\hline
\end{tabular}




\begin{tabular}{|c|c|c|c|c|c|c|c|c|c|c|c|c|c|c|c|c|c|}
\hline FA (20:3) & 306.3 & 7.3 & $\mathrm{C} 20 \mathrm{H} 34 \mathrm{O} 2$ & $\begin{array}{l}\text { Fatty } \\
\text { Acyls }\end{array}$ & $\begin{array}{l}\text { Fatty } \\
\text { Acids and } \\
\text { Conjugates }\end{array}$ & $\begin{array}{l}10261 \\
34.5\end{array}$ & $\begin{array}{l}153097 \\
9.5\end{array}$ & $\begin{array}{l}353591 \\
1.5\end{array}$ & $\begin{array}{l}409056 \\
4.5\end{array}$ & $\begin{array}{l}499578 \\
2.5\end{array}$ & $\begin{array}{l}112994 \\
6.9\end{array}$ & 2943.4 & 2175.1 & 1783.6 & 3095.6 & 0.0 & 0.0 \\
\hline FA (20:4) & 304.2 & 6.9 & $\mathrm{C} 2 \mathrm{OH} 32 \mathrm{O} 2$ & $\begin{array}{l}\text { Fatty } \\
\text { Acyls }\end{array}$ & $\begin{array}{l}\text { Fatty } \\
\text { Acids and } \\
\text { Conjugates }\end{array}$ & $\begin{array}{l}15237 \\
12.4\end{array}$ & $\begin{array}{l}240761 \\
9.0\end{array}$ & $\begin{array}{l}504320 \\
7.5\end{array}$ & $\begin{array}{l}607904 \\
9.5\end{array}$ & $\begin{array}{l}751232 \\
4.5\end{array}$ & $\begin{array}{l}153774 \\
7.8\end{array}$ & 0.0 & 0.0 & 0.0 & 0.0 & 0.0 & 0.0 \\
\hline FA (30:0) & 469.5 & 19.0 & $\mathrm{C} 30 \mathrm{H} 60 \mathrm{O} 2$ & $\begin{array}{l}\text { Fatty } \\
\text { Acyls }\end{array}$ & $\begin{array}{l}\text { Fatty } \\
\text { Acids and } \\
\text { Conjugates }\end{array}$ & $\begin{array}{l}66032 \\
.7\end{array}$ & $\begin{array}{l}112911 \\
.2\end{array}$ & $\begin{array}{l}37954 . \\
3\end{array}$ & $\begin{array}{l}57654 . \\
4\end{array}$ & $\begin{array}{l}18618 \\
4\end{array}$ & $\begin{array}{l}84444 . \\
9\end{array}$ & $\begin{array}{l}14329 . \\
3\end{array}$ & 7928.9 & 4250.5 & 5988.9 & 6259.3 & 6669.8 \\
\hline FA $(9: 1)$ nonenal & 157.1 & 2.1 & $\mathrm{C} 9 \mathrm{H} 16 \mathrm{O}$ & $\begin{array}{l}\text { Fatty } \\
\text { Acyls }\end{array}$ & $\begin{array}{l}\text { Fatty } \\
\text { Acids and } \\
\text { Conjugates }\end{array}$ & $\begin{array}{l}42691 \\
.2\end{array}$ & $\begin{array}{l}23462 . \\
5\end{array}$ & $\begin{array}{l}46570 . \\
7\end{array}$ & $\begin{array}{l}34716 . \\
7\end{array}$ & $\begin{array}{l}81416 . \\
1\end{array}$ & $\begin{array}{l}54661 . \\
2\end{array}$ & $\begin{array}{l}32133 . \\
9\end{array}$ & $\begin{array}{l}72643 . \\
6\end{array}$ & $\begin{array}{l}52005 . \\
3\end{array}$ & $\begin{array}{l}50116 . \\
2\end{array}$ & $\begin{array}{l}61437 . \\
0\end{array}$ & $\begin{array}{l}51621 . \\
7\end{array}$ \\
\hline FA hydroxy(12:1) & 178.1 & 5.5 & $\mathrm{C} 10 \mathrm{H} 10 \mathrm{O} 3$ & $\begin{array}{l}\text { Fatty } \\
\text { Acyls }\end{array}$ & $\begin{array}{l}\text { Fatty } \\
\text { Acids and } \\
\text { Conjugates }\end{array}$ & 0.0 & 0.0 & 0.0 & 0.0 & 0.0 & 0.0 & $\begin{array}{l}236416 \\
.9\end{array}$ & $\begin{array}{l}194586 \\
.0\end{array}$ & $\begin{array}{l}170031 \\
.1\end{array}$ & $\begin{array}{l}155682 \\
.3\end{array}$ & $\begin{array}{l}190763 \\
.1\end{array}$ & $\begin{array}{l}142323 \\
.9\end{array}$ \\
\hline FA methyl(14:0) & 242.2 & 6.5 & $\mathrm{C} 15 \mathrm{H} 30 \mathrm{O} 2$ & $\begin{array}{l}\text { Fatty } \\
\text { Acyls }\end{array}$ & $\begin{array}{l}\text { Fatty } \\
\text { Acids and } \\
\text { Conjugates }\end{array}$ & $\begin{array}{l}50432 \\
.9\end{array}$ & $\begin{array}{l}68180 . \\
1\end{array}$ & $\begin{array}{l}98705 . \\
5\end{array}$ & $\begin{array}{l}162766 \\
.2\end{array}$ & $\begin{array}{l}234752 \\
.2\end{array}$ & $\begin{array}{l}97818 . \\
2\end{array}$ & 0.0 & 0.0 & 0.0 & 0.0 & 0.0 & 0.0 \\
\hline FA methyl(16:1) & 268.2 & 7.1 & $\mathrm{C} 17 \mathrm{H} 32 \mathrm{O} 2$ & $\begin{array}{l}\text { Fatty } \\
\text { Acyls }\end{array}$ & $\begin{array}{l}\text { Fatty } \\
\text { Acids and } \\
\text { Conjugates }\end{array}$ & $\begin{array}{l}66692 \\
.0\end{array}$ & $\begin{array}{l}94620 . \\
3\end{array}$ & $\begin{array}{l}194750 \\
.0\end{array}$ & $\begin{array}{l}276979 \\
.6\end{array}$ & $\begin{array}{l}309533 \\
.7\end{array}$ & $\begin{array}{l}190267 \\
.8\end{array}$ & 0.0 & 0.0 & 0.0 & 0.0 & 0.0 & 0.0 \\
\hline FA methyl(18:0) & 298.3 & 9.4 & $\mathrm{C} 19 \mathrm{H} 38 \mathrm{O} 2$ & $\begin{array}{l}\text { Fatty } \\
\text { Acyls }\end{array}$ & $\begin{array}{l}\text { Fatty } \\
\text { Acids and } \\
\text { Conjugates }\end{array}$ & $\begin{array}{l}34906 \\
.9\end{array}$ & $\begin{array}{l}38746 \\
4\end{array}$ & $\begin{array}{l}59290 . \\
5\end{array}$ & $\begin{array}{l}74529 . \\
6\end{array}$ & $\begin{array}{l}170690 \\
.1\end{array}$ & $\begin{array}{l}42732 \\
4\end{array}$ & $\begin{array}{l}14710 . \\
7\end{array}$ & $\begin{array}{l}16133 \\
4\end{array}$ & $\begin{array}{l}13659 \\
8\end{array}$ & $\begin{array}{l}17496 \\
8\end{array}$ & $\begin{array}{l}27221 \\
8\end{array}$ & $\begin{array}{l}12029 . \\
3\end{array}$ \\
\hline $\begin{array}{l}\text { FA oxo(14:1) } \\
\text { cyclopentanebutanoic acid }\end{array}$ & 238.2 & 3.3 & $\mathrm{C} 14 \mathrm{H} 22 \mathrm{O} 3$ & $\begin{array}{l}\text { Fatty } \\
\text { Acyls }\end{array}$ & $\begin{array}{l}\text { Octadecan } \\
\text { oids }\end{array}$ & 0.0 & 0.0 & 0.0 & 0.0 & 0.0 & 0.0 & 0.0 & 0.0 & 5449.1 & 0.0 & 9243.8 & 8220.1 \\
\hline FA oxo(16:0) & 270.2 & 3.6 & $\mathrm{C} 16 \mathrm{H} 30 \mathrm{O} 3$ & $\begin{array}{l}\text { Fatty } \\
\text { Acyls }\end{array}$ & $\begin{array}{l}\text { Fatty } \\
\text { Acids and } \\
\text { Conjugates }\end{array}$ & $\begin{array}{l}25769 \\
.7\end{array}$ & $\begin{array}{l}27761 . \\
9\end{array}$ & $\begin{array}{l}66322 . \\
4\end{array}$ & $\begin{array}{l}64431 \\
7\end{array}$ & $\begin{array}{l}128475 \\
.5\end{array}$ & $\begin{array}{l}46375 \\
7\end{array}$ & 0.0 & 0.0 & 0.0 & 0.0 & 0.0 & 0.0 \\
\hline FA oxo(21:0) & 340.3 & 15.4 & $\mathrm{C} 21 \mathrm{H} 40 \mathrm{O} 3$ & $\begin{array}{l}\text { Fatty } \\
\text { Acyls }\end{array}$ & $\begin{array}{l}\text { Fatty } \\
\text { Acids and } \\
\text { Conjugates }\end{array}$ & $\begin{array}{l}32371 \\
.0\end{array}$ & $\begin{array}{l}52560 . \\
8\end{array}$ & $\begin{array}{l}62050 . \\
4\end{array}$ & $\begin{array}{l}78562 . \\
0\end{array}$ & $\begin{array}{l}36592 . \\
9\end{array}$ & $\begin{array}{l}60220 \\
5\end{array}$ & $\begin{array}{l}27951 . \\
1\end{array}$ & $\begin{array}{l}29880 . \\
6\end{array}$ & $\begin{array}{l}36672 . \\
7\end{array}$ & $\begin{array}{l}30556 . \\
3\end{array}$ & $\begin{array}{l}34699 . \\
1\end{array}$ & $\begin{array}{l}38009 . \\
5\end{array}$ \\
\hline $\mathrm{FA}(16: 1)$ & 254.2 & 6.4 & $\mathrm{C} 16 \mathrm{H} 30 \mathrm{O} 2$ & $\begin{array}{l}\text { Lipid } \\
\text { Metabolis } \\
\mathrm{m}\end{array}$ & $\begin{array}{l}\text { Fatty acid } \\
\text { biosynthes } \\
\text { is }\end{array}$ & $\begin{array}{l}33946 \\
.2\end{array}$ & $\begin{array}{l}39090 . \\
5\end{array}$ & $\begin{array}{l}32278 \\
4\end{array}$ & $\begin{array}{l}66061 \\
2\end{array}$ & $\begin{array}{l}120038 \\
.6\end{array}$ & $\begin{array}{l}371867 \\
.7\end{array}$ & 0.0 & 0.0 & 0.0 & 0.0 & 0.0 & 0.0 \\
\hline $\begin{array}{l}\text { Ganglioside GM3 } \\
\text { (d18:0/16:0) }\end{array}$ & 1154.7 & 11.3 & $\begin{array}{l}\text { C57H106N2O } \\
21\end{array}$ & $\begin{array}{l}\text { Gangliosi } \\
\text { des }\end{array}$ & na & $\begin{array}{l}34340 \\
.7\end{array}$ & $\begin{array}{l}11347 . \\
7\end{array}$ & 0.0 & 4928.4 & 0.0 & 0.0 & 0.0 & 0.0 & 0.0 & 0.0 & 0.0 & 0.0 \\
\hline
\end{tabular}




\begin{tabular}{|c|c|c|c|c|c|c|c|c|c|c|c|c|c|c|c|c|c|}
\hline GL amino(36:0) & 785.6 & 13.3 & $\mathrm{C} 45 \mathrm{H} 87 \mathrm{NO} 9$ & $\begin{array}{l}\text { Glyceroli } \\
\text { pids }\end{array}$ & $\begin{array}{l}\text { Glycosyldi } \\
\text { radylglyce } \\
\text { rols }\end{array}$ & $\begin{array}{l}20884 \\
5.2\end{array}$ & $\begin{array}{l}420523 \\
.8\end{array}$ & $\begin{array}{l}588477 \\
.9\end{array}$ & $\begin{array}{l}697626 \\
.6\end{array}$ & $\begin{array}{l}632700 \\
.0\end{array}$ & $\begin{array}{l}729180 \\
.5\end{array}$ & 0.0 & 0.0 & 0.0 & 0.0 & 0.0 & 0.0 \\
\hline GlcCer(d18:2/20:0) & 753.6 & 13.1 & C44H83NO8 & $\begin{array}{l}\text { Sphingoli } \\
\text { pids }\end{array}$ & na & $\begin{array}{l}85893 \\
.5\end{array}$ & $\begin{array}{l}108279 \\
.4\end{array}$ & $\begin{array}{l}111861 \\
.2\end{array}$ & $\begin{array}{l}115980 \\
.4\end{array}$ & $\begin{array}{l}120018 \\
.0\end{array}$ & $\begin{array}{l}153271 \\
.8\end{array}$ & 0.0 & 0.0 & 0.0 & 0.0 & 0.0 & 0.0 \\
\hline GlcCer(d18:2/21:0) & 767.6 & 13.8 & C45H85NO8 & $\begin{array}{l}\text { Sphingoli } \\
\text { pids }\end{array}$ & na & $\begin{array}{l}16958 \\
0.5\end{array}$ & $\begin{array}{l}238460 \\
.5\end{array}$ & $\begin{array}{l}304210 \\
.6\end{array}$ & $\begin{array}{l}273017 \\
.1\end{array}$ & $\begin{array}{l}252206 \\
.8\end{array}$ & $\begin{array}{l}246514 \\
.0\end{array}$ & $\begin{array}{l}183726 \\
.6\end{array}$ & $\begin{array}{l}193308 \\
.6\end{array}$ & $\begin{array}{l}194352 \\
.9\end{array}$ & $\begin{array}{l}187486 \\
.5\end{array}$ & $\begin{array}{l}188429 \\
.4\end{array}$ & $\begin{array}{l}195521 \\
.1\end{array}$ \\
\hline Heptacosene & 378.4 & 18.3 & $\mathrm{C} 27 \mathrm{H} 54$ & $\begin{array}{l}\text { Fatty } \\
\text { Acyls }\end{array}$ & $\begin{array}{l}\text { Hydrocarb } \\
\text { ons }\end{array}$ & $\begin{array}{l}5694 . \\
8\end{array}$ & 7269.9 & 0.0 & 0.0 & 0.0 & 0.0 & 0.0 & 0.0 & 0.0 & 0.0 & 0.0 & 0.0 \\
\hline Hexadecanoic acid & 256.2 & 7.3 & $\mathrm{C} 16 \mathrm{H} 32 \mathrm{O} 2$ & $\begin{array}{l}\text { Lipid } \\
\text { Metabolis } \\
\mathrm{m}\end{array}$ & $\begin{array}{l}\text { Fatty acid } \\
\text { biosynthes } \\
\text { is_Fatty } \\
\text { acid } \\
\text { elongation } \\
\text { in } \\
\text { mitochond } \\
\text { ria_Fatty } \\
\text { acid } \\
\text { metabolis } \\
\text { m_Biosy } \\
\text { nthesis of } \\
\text { unsaturate } \\
\text { d fatty } \\
\text { acids }\end{array}$ & $\begin{array}{l}48018 \\
40.0\end{array}$ & $\begin{array}{l}340122 \\
7.8\end{array}$ & $\begin{array}{l}104932 \\
94.0\end{array}$ & $\begin{array}{l}124068 \\
99.0\end{array}$ & $\begin{array}{l}176708 \\
74.0\end{array}$ & $\begin{array}{l}803923 \\
8.5\end{array}$ & $\begin{array}{l}653974 \\
7.0\end{array}$ & $\begin{array}{l}605450 \\
8.0\end{array}$ & $\begin{array}{l}597506 \\
5.5\end{array}$ & $\begin{array}{l}605306 \\
1.5\end{array}$ & $\begin{array}{l}731175 \\
7.5\end{array}$ & $\begin{array}{l}432840 \\
9.5\end{array}$ \\
\hline $\begin{array}{l}\text { Hydroxyphthioceranic acid } \\
\text { (C31) }\end{array}$ & 482.5 & 14.1 & $\mathrm{C} 31 \mathrm{H} 62 \mathrm{O} 3$ & $\begin{array}{l}\text { Fatty } \\
\text { Acyls }\end{array}$ & $\begin{array}{l}\text { Fatty } \\
\text { Acids and } \\
\text { Conjugates }\end{array}$ & $\begin{array}{l}95889 \\
.3\end{array}$ & $\begin{array}{l}161170 \\
.9\end{array}$ & $\begin{array}{l}217751 \\
.8\end{array}$ & $\begin{array}{l}222168 \\
.4\end{array}$ & $\begin{array}{l}85748 . \\
3\end{array}$ & $\begin{array}{l}233852 \\
.4\end{array}$ & 0.0 & 0.0 & 0.0 & 0.0 & 0.0 & 0.0 \\
\hline $\begin{array}{l}\text { Hydroxyphthioceranic acid } \\
\text { (C33) }\end{array}$ & 510.5 & 15.6 & $\mathrm{C} 33 \mathrm{H} 66 \mathrm{O} 3$ & $\begin{array}{l}\text { Fatty } \\
\text { Acyls }\end{array}$ & $\begin{array}{l}\text { Fatty } \\
\text { Acids and } \\
\text { Conjugates }\end{array}$ & $\begin{array}{l}63869 \\
.7\end{array}$ & $\begin{array}{l}103736 \\
.1\end{array}$ & $\begin{array}{l}116060 \\
.0\end{array}$ & $\begin{array}{l}140283 \\
.4\end{array}$ & $\begin{array}{l}37957 . \\
3\end{array}$ & $\begin{array}{l}118811 \\
.8\end{array}$ & 0.0 & 0.0 & 0.0 & 0.0 & 0.0 & 0.0 \\
\hline $\begin{array}{l}\text { Hydroxyphthioceranic acid } \\
\text { (C37) }\end{array}$ & 566.6 & 18.5 & $\mathrm{C} 37 \mathrm{H} 74 \mathrm{O} 3$ & $\begin{array}{l}\text { Fatty } \\
\text { Acyls }\end{array}$ & $\begin{array}{l}\text { Fatty } \\
\text { Acids and } \\
\text { Conjugates }\end{array}$ & $\begin{array}{l}27640 \\
.8\end{array}$ & $\begin{array}{l}34580 . \\
4\end{array}$ & $\begin{array}{l}25110 . \\
3\end{array}$ & $\begin{array}{l}28707 . \\
4\end{array}$ & $\begin{array}{l}11742 . \\
6\end{array}$ & $\begin{array}{l}51088 . \\
8\end{array}$ & 0.0 & 0.0 & 0.0 & 0.0 & 0.0 & 0.0 \\
\hline Linoleamide & 279.3 & 5.8 & $\mathrm{C} 18 \mathrm{H} 33 \mathrm{NO}$ & $\begin{array}{l}\text { Fatty } \\
\text { Acyls }\end{array}$ & $\begin{array}{l}\text { Fatty } \\
\text { amides }\end{array}$ & $\begin{array}{l}24102 \\
6.8\end{array}$ & $\begin{array}{l}186362 \\
.9\end{array}$ & $\begin{array}{l}260830 \\
.3\end{array}$ & $\begin{array}{l}566143 \\
.3\end{array}$ & $\begin{array}{l}354616 \\
.8\end{array}$ & $\begin{array}{l}328508 \\
.7\end{array}$ & 0.0 & 0.0 & 0.0 & 0.0 & 0.0 & 0.0 \\
\hline Lipoxin D4 & 529.3 & 4.3 & $\begin{array}{l}\mathrm{C} 25 \mathrm{H} 40 \mathrm{~N} 2 \mathrm{O} 7 \\
\mathrm{~S}\end{array}$ & $\begin{array}{l}\text { Fatty } \\
\text { Acyls }\end{array}$ & $\begin{array}{l}\text { Eicosanoid } \\
\mathrm{s}\end{array}$ & $\begin{array}{l}30762 \\
.5\end{array}$ & $\begin{array}{l}367834 \\
.7\end{array}$ & $\begin{array}{l}87381 . \\
9\end{array}$ & $\begin{array}{l}62861 . \\
5\end{array}$ & $\begin{array}{l}54039 . \\
3\end{array}$ & $\begin{array}{l}67392 . \\
9\end{array}$ & 6030.1 & 0.0 & 0.0 & 0.0 & 0.0 & 0.0 \\
\hline
\end{tabular}




\begin{tabular}{|c|c|c|c|c|c|c|c|c|c|c|c|c|c|c|c|c|c|}
\hline LysoPC(12:0) & 439.3 & 3.6 & $\mathrm{C} 20 \mathrm{H} 42 \mathrm{NO} 7 \mathrm{P}$ & $\begin{array}{l}\text { Glyceroph } \\
\text { ospholipid } \\
\text { s }\end{array}$ & $\begin{array}{l}\text { Glyceroph } \\
\text { osphocholi } \\
\text { nes }\end{array}$ & $\begin{array}{l}26715 \\
3.6\end{array}$ & $\begin{array}{l}321151 \\
.2\end{array}$ & $\begin{array}{l}336405 \\
.9\end{array}$ & $\begin{array}{l}250664 \\
.1\end{array}$ & $\begin{array}{l}206418 \\
.9\end{array}$ & $\begin{array}{l}210734 \\
.8\end{array}$ & 0.0 & 0.0 & 0.0 & 0.0 & 0.0 & 0.0 \\
\hline LysoPC(16:0) & 495.3 & 6.5 & $\mathrm{C} 24 \mathrm{H} 50 \mathrm{NO} 7 \mathrm{P}$ & $\begin{array}{l}\text { Glyceroph } \\
\text { ospholipid } \\
\text { s }\end{array}$ & $\begin{array}{l}\text { Glyceroph } \\
\text { osphocholi } \\
\text { nes }\end{array}$ & $\begin{array}{l}63992 \\
.2\end{array}$ & $\begin{array}{l}102604 \\
.9\end{array}$ & $\begin{array}{l}152768 \\
.8\end{array}$ & $\begin{array}{l}173734 \\
.1\end{array}$ & $\begin{array}{l}194588 \\
.7\end{array}$ & $\begin{array}{l}103410 \\
.8\end{array}$ & 0.0 & 0.0 & 0.0 & 0.0 & 0.0 & 0.0 \\
\hline LysoPC(18:0) & 523.4 & 6.1 & $\mathrm{C} 26 \mathrm{H} 54 \mathrm{NO} 7 \mathrm{P}$ & $\begin{array}{l}\text { Glyceroph } \\
\text { ospholipid } \\
\text { s }\end{array}$ & $\begin{array}{l}\text { Glyceroph } \\
\text { osphocholi } \\
\text { nes }\end{array}$ & $\begin{array}{l}14895 \\
8.6\end{array}$ & $\begin{array}{l}275348 \\
.5\end{array}$ & $\begin{array}{l}469809 \\
.4\end{array}$ & $\begin{array}{l}531313 \\
.4\end{array}$ & $\begin{array}{l}650557 \\
.0\end{array}$ & $\begin{array}{l}274956 \\
.5\end{array}$ & 9033.8 & 8632.4 & 8208.3 & 7055.9 & 8079.2 & 7089.6 \\
\hline LysoPC(20:1) & 549.4 & 6.2 & $\mathrm{C} 28 \mathrm{H} 56 \mathrm{NO} 7 \mathrm{P}$ & $\begin{array}{l}\text { Glyceroph } \\
\text { ospholipid } \\
\text { s }\end{array}$ & $\begin{array}{l}\text { Glyceroph } \\
\text { osphocholi } \\
\text { nes }\end{array}$ & $\begin{array}{l}13167 \\
.1\end{array}$ & $\begin{array}{l}17973 . \\
8\end{array}$ & $\begin{array}{l}26278 \\
3\end{array}$ & $\begin{array}{l}34209 . \\
7\end{array}$ & $\begin{array}{l}45283 . \\
2\end{array}$ & $\begin{array}{l}11686 . \\
4\end{array}$ & 0.0 & 0.0 & 0.0 & 0.0 & 0.0 & 0.0 \\
\hline LysoPC(20:2) & 547.4 & 5.4 & $\mathrm{C} 28 \mathrm{H} 54 \mathrm{NO} 7 \mathrm{P}$ & $\begin{array}{l}\text { Glyceroph } \\
\text { ospholipid } \\
\text { s }\end{array}$ & $\begin{array}{l}\text { Glyceroph } \\
\text { osphocholi } \\
\text { nes }\end{array}$ & $\begin{array}{l}26069 \\
.6\end{array}$ & $\begin{array}{l}32767 . \\
8\end{array}$ & $\begin{array}{l}52102 . \\
2\end{array}$ & $\begin{array}{l}53566 . \\
5\end{array}$ & $\begin{array}{l}62491 . \\
2\end{array}$ & $\begin{array}{l}27727 . \\
0\end{array}$ & 0.0 & 0.0 & 0.0 & 0.0 & 0.0 & 0.0 \\
\hline LysoPC(20:3) & 545.3 & 4.7 & $\mathrm{C} 28 \mathrm{H} 52 \mathrm{NO} 7 \mathrm{P}$ & $\begin{array}{l}\text { Glyceroph } \\
\text { ospholipid } \\
\text { s }\end{array}$ & $\begin{array}{l}\text { Glyceroph } \\
\text { osphocholi } \\
\text { nes }\end{array}$ & $\begin{array}{l}21832 \\
6.5\end{array}$ & $\begin{array}{l}294954 \\
.9\end{array}$ & $\begin{array}{l}474895 \\
.0\end{array}$ & $\begin{array}{l}474997 \\
.0\end{array}$ & $\begin{array}{l}497827 \\
.8\end{array}$ & $\begin{array}{l}262785 \\
.8\end{array}$ & 0.0 & 0.0 & 0.0 & 0.0 & 0.0 & 0.0 \\
\hline LysoPC(20:4) & 543.3 & 4.3 & $\mathrm{C} 28 \mathrm{H} 50 \mathrm{NO} 7 \mathrm{P}$ & $\begin{array}{l}\text { Glyceroph } \\
\text { ospholipid } \\
\text { s }\end{array}$ & $\begin{array}{l}\text { Glyceroph } \\
\text { osphocholi } \\
\text { nes }\end{array}$ & $\begin{array}{l}80272 \\
.7\end{array}$ & $\begin{array}{l}95206 . \\
0\end{array}$ & $\begin{array}{l}117153 \\
.2\end{array}$ & $\begin{array}{l}111660 \\
.5\end{array}$ & $\begin{array}{l}94494 . \\
1\end{array}$ & $\begin{array}{l}64980 . \\
0\end{array}$ & 0.0 & 0.0 & 0.0 & 0.0 & 0.0 & 0.0 \\
\hline LysoPC(O-16:1) & 479.3 & 7.2 & $\mathrm{C} 24 \mathrm{H} 50 \mathrm{NO} 6 \mathrm{P}$ & $\begin{array}{l}\text { Glyceroph } \\
\text { ospholipid } \\
\text { s }\end{array}$ & $\begin{array}{l}\text { Glyceroph } \\
\text { osphocholi } \\
\text { nes }\end{array}$ & $\begin{array}{l}67061 \\
.5\end{array}$ & $\begin{array}{l}93487 . \\
5\end{array}$ & $\begin{array}{l}226690 \\
.6\end{array}$ & $\begin{array}{l}271736 \\
.2\end{array}$ & $\begin{array}{l}389459 \\
.3\end{array}$ & $\begin{array}{l}99582 . \\
4\end{array}$ & 0.0 & 0.0 & 0.0 & 0.0 & 0.0 & 0.0 \\
\hline LysoPC(O-18:2) & 509.4 & 6.7 & $\mathrm{C} 26 \mathrm{H} 56 \mathrm{NO} 6 \mathrm{P}$ & $\begin{array}{l}\text { Glyceroph } \\
\text { ospholipid } \\
\text { s }\end{array}$ & $\begin{array}{l}\text { Glyceroph } \\
\text { osphocholi } \\
\text { nes }\end{array}$ & $\begin{array}{l}97869 \\
.4\end{array}$ & $\begin{array}{l}199813 \\
.1\end{array}$ & $\begin{array}{l}268772 \\
.9\end{array}$ & $\begin{array}{l}366631 \\
.3\end{array}$ & $\begin{array}{l}698209 \\
.8\end{array}$ & $\begin{array}{l}224759 \\
.1\end{array}$ & 0.0 & 0.0 & 0.0 & 0.0 & 0.0 & 0.0 \\
\hline LysoPE(16:0) & 453.3 & 4.5 & $\mathrm{C} 21 \mathrm{H} 44 \mathrm{NO} 7 \mathrm{P}$ & $\begin{array}{l}\text { Glyceroph } \\
\text { ospholipid } \\
\text { s }\end{array}$ & $\begin{array}{l}\text { Glyceroph } \\
\text { osphoetha } \\
\text { nolamines }\end{array}$ & $\begin{array}{l}80328 \\
.0\end{array}$ & $\begin{array}{l}128722 \\
.1\end{array}$ & $\begin{array}{l}192266 \\
.9\end{array}$ & $\begin{array}{l}195031 \\
.5\end{array}$ & $\begin{array}{l}245526 \\
.3\end{array}$ & $\begin{array}{l}101648 \\
.4\end{array}$ & 0.0 & 0.0 & 0.0 & 0.0 & 0.0 & 0.0 \\
\hline LysoPE(18:0) & 527.3 & 3.8 & $\mathrm{C} 23 \mathrm{H} 48 \mathrm{NO} 7 \mathrm{P}$ & $\begin{array}{l}\text { Glyceroph } \\
\text { ospholipid } \\
\text { s }\end{array}$ & $\begin{array}{l}\text { Glyceroph } \\
\text { osphoetha } \\
\text { nolamines }\end{array}$ & $\begin{array}{l}14495 \\
99.6\end{array}$ & $\begin{array}{l}308155 \\
9.5\end{array}$ & $\begin{array}{l}495109 \\
0.0\end{array}$ & $\begin{array}{l}561288 \\
8.0\end{array}$ & $\begin{array}{l}593678 \\
3.0\end{array}$ & $\begin{array}{l}228141 \\
2.3\end{array}$ & 0.0 & 0.0 & 0.0 & 0.0 & 0.0 & 0.0 \\
\hline LysoPE(18:1) & 465.3 & 6.5 & $\mathrm{C} 23 \mathrm{H} 48 \mathrm{NO} 6 \mathrm{P}$ & $\begin{array}{l}\text { Glyceroph } \\
\text { ospholipid } \\
\text { s }\end{array}$ & $\begin{array}{l}\text { Glyceroph } \\
\text { osphoetha } \\
\text { nolamines }\end{array}$ & $\begin{array}{l}15705 \\
96.6\end{array}$ & $\begin{array}{l}265396 \\
7.3\end{array}$ & $\begin{array}{l}508654 \\
0.5\end{array}$ & $\begin{array}{l}742059 \\
2.0\end{array}$ & $\begin{array}{l}854003 \\
3.0\end{array}$ & $\begin{array}{l}215150 \\
7.0\end{array}$ & 0.0 & 0.0 & 0.0 & 0.0 & 0.0 & 0.0 \\
\hline LysoPE(18:2) & 477.3 & 4.0 & $\mathrm{C} 23 \mathrm{H} 44 \mathrm{NO} 7 \mathrm{P}$ & $\begin{array}{l}\text { Glyceroph } \\
\text { ospholipid } \\
\mathrm{s}\end{array}$ & $\begin{array}{l}\text { Glyceroph } \\
\text { osphoetha } \\
\text { nolamines }\end{array}$ & $\begin{array}{l}16089 \\
5.8\end{array}$ & $\begin{array}{l}184856 \\
.9\end{array}$ & $\begin{array}{l}220663 \\
.6\end{array}$ & $\begin{array}{l}152238 \\
.1\end{array}$ & $\begin{array}{l}79180 . \\
6\end{array}$ & $\begin{array}{l}154562 \\
.4\end{array}$ & 0.0 & 0.0 & 0.0 & 0.0 & 0.0 & 0.0 \\
\hline
\end{tabular}




\begin{tabular}{|c|c|c|c|c|c|c|c|c|c|c|c|c|c|c|c|c|c|}
\hline LysoPE(20:0) & 509.3 & 7.2 & $\mathrm{C} 25 \mathrm{H} 52 \mathrm{NO} 7 \mathrm{P}$ & $\begin{array}{l}\text { Glyceroph } \\
\text { ospholipid } \\
\mathrm{s}\end{array}$ & $\begin{array}{l}\text { Glyceroph } \\
\text { osphoetha } \\
\text { nolamines }\end{array}$ & $\begin{array}{l}98609 \\
.2\end{array}$ & $\begin{array}{l}186543 \\
.1\end{array}$ & $\begin{array}{l}263877 \\
.3\end{array}$ & $\begin{array}{l}262434 \\
.9\end{array}$ & $\begin{array}{l}313988 \\
.4\end{array}$ & $\begin{array}{l}166296 \\
.4\end{array}$ & 0.0 & 0.0 & 0.0 & 0.0 & 0.0 & 0.0 \\
\hline LysoPE(20:1) & 507.3 & 6.1 & $\mathrm{C} 25 \mathrm{H} 50 \mathrm{NO} 7 \mathrm{P}$ & $\begin{array}{l}\text { Glyceroph } \\
\text { ospholipid } \\
\mathrm{s}\end{array}$ & $\begin{array}{l}\text { Glyceroph } \\
\text { osphoetha } \\
\text { nolamines }\end{array}$ & $\begin{array}{l}94622 \\
.2\end{array}$ & $\begin{array}{l}174008 \\
.8\end{array}$ & $\begin{array}{l}340004 \\
.1\end{array}$ & $\begin{array}{l}355011 \\
.0\end{array}$ & $\begin{array}{l}252475 \\
.2\end{array}$ & $\begin{array}{l}232765 \\
.7\end{array}$ & 0.0 & 0.0 & 0.0 & 0.0 & 0.0 & 0.0 \\
\hline LysoPE(20:2) & 505.3 & 5.2 & $\mathrm{C} 25 \mathrm{H} 48 \mathrm{NO} 7 \mathrm{P}$ & $\begin{array}{l}\text { Glyceroph } \\
\text { ospholipid } \\
\mathrm{s}\end{array}$ & $\begin{array}{l}\text { Glyceroph } \\
\text { osphoetha } \\
\text { nolamines }\end{array}$ & $\begin{array}{l}60174 \\
.8\end{array}$ & $\begin{array}{l}77497 . \\
2\end{array}$ & $\begin{array}{l}105055 \\
.4\end{array}$ & $\begin{array}{l}74748 . \\
3\end{array}$ & $\begin{array}{l}57113 . \\
3\end{array}$ & $\begin{array}{l}65292 . \\
6\end{array}$ & 0.0 & 0.0 & 0.0 & 0.0 & 0.0 & 0.0 \\
\hline LysoPE(20:3) & 503.3 & 4.5 & $\mathrm{C} 25 \mathrm{H} 46 \mathrm{NO} 7 \mathrm{P}$ & $\begin{array}{l}\text { Glyceroph } \\
\text { ospholipid } \\
\text { s }\end{array}$ & $\begin{array}{l}\text { Glyceroph } \\
\text { osphoetha } \\
\text { nolamines }\end{array}$ & $\begin{array}{l}17188 \\
9.6\end{array}$ & $\begin{array}{l}192597 \\
.7\end{array}$ & $\begin{array}{l}306361 \\
.7\end{array}$ & $\begin{array}{l}224066 \\
.9\end{array}$ & $\begin{array}{l}146018 \\
.2\end{array}$ & $\begin{array}{l}195573 \\
.9\end{array}$ & 0.0 & 0.0 & 0.0 & 0.0 & 0.0 & 0.0 \\
\hline LysoPE(20:4) & 501.3 & 4.2 & $\mathrm{C} 25 \mathrm{H} 44 \mathrm{NO} 7 \mathrm{P}$ & $\begin{array}{l}\text { Glyceroph } \\
\text { ospholipid } \\
\text { s }\end{array}$ & $\begin{array}{l}\text { Glyceroph } \\
\text { osphoetha } \\
\text { nolamines }\end{array}$ & $\begin{array}{l}29723 \\
1.0\end{array}$ & $\begin{array}{l}323377 \\
.5\end{array}$ & $\begin{array}{l}458928 \\
.7\end{array}$ & $\begin{array}{l}282207 \\
.6\end{array}$ & $\begin{array}{l}127382 \\
.0\end{array}$ & $\begin{array}{l}297373 \\
.4\end{array}$ & 0.0 & 0.0 & 0.0 & 0.0 & 0.0 & 0.0 \\
\hline LysoPE(22:0) & 537.4 & 6.7 & $\mathrm{C} 27 \mathrm{H} 56 \mathrm{NO} 7 \mathrm{P}$ & $\begin{array}{l}\text { Glyceroph } \\
\text { ospholipid } \\
\mathrm{s}\end{array}$ & $\begin{array}{l}\text { Glyceroph } \\
\text { osphoetha } \\
\text { nolamines }\end{array}$ & $\begin{array}{l}11740 \\
.9\end{array}$ & $\begin{array}{l}18331 . \\
1\end{array}$ & $\begin{array}{l}31858 . \\
7\end{array}$ & $\begin{array}{l}34160 . \\
5\end{array}$ & $\begin{array}{l}69482 . \\
4\end{array}$ & $\begin{array}{l}20655 . \\
0\end{array}$ & 0.0 & 0.0 & 0.0 & 0.0 & 0.0 & 0.0 \\
\hline LysoPE(22:1) & 535.4 & 5.7 & $\mathrm{C} 27 \mathrm{H} 54 \mathrm{NO} 7 \mathrm{P}$ & $\begin{array}{l}\text { Glyceroph } \\
\text { ospholipid } \\
\mathrm{s}\end{array}$ & $\begin{array}{l}\text { Glyceroph } \\
\text { osphoetha } \\
\text { nolamines }\end{array}$ & $\begin{array}{l}24739 \\
.1\end{array}$ & $\begin{array}{l}42894 . \\
7\end{array}$ & $\begin{array}{l}82586 . \\
7\end{array}$ & $\begin{array}{l}137295 \\
.3\end{array}$ & $\begin{array}{l}235280 \\
.0\end{array}$ & $\begin{array}{l}38637 . \\
3\end{array}$ & 0.0 & 0.0 & 0.0 & 0.0 & 0.0 & 0.0 \\
\hline LysoPE(22:2) & 533.3 & 4.8 & $\mathrm{C} 27 \mathrm{H} 52 \mathrm{NO} 7 \mathrm{P}$ & $\begin{array}{l}\text { Glyceroph } \\
\text { ospholipid } \\
\mathrm{s}\end{array}$ & $\begin{array}{l}\text { Glyceroph } \\
\text { osphoetha } \\
\text { nolamines }\end{array}$ & $\begin{array}{l}2261 . \\
5\end{array}$ & 2246.3 & 3304.3 & 2939.8 & 4998.8 & 0.0 & 0.0 & 0.0 & 0.0 & 0.0 & 0.0 & 0.0 \\
\hline LysoPE(22:4) & 529.3 & 4.1 & $\mathrm{C} 27 \mathrm{H} 48 \mathrm{NO} 7 \mathrm{P}$ & $\begin{array}{l}\text { Glyceroph } \\
\text { ospholipid } \\
\mathrm{s}\end{array}$ & $\begin{array}{l}\text { Glyceroph } \\
\text { osphoetha } \\
\text { nolamines }\end{array}$ & $\begin{array}{l}5404 . \\
1\end{array}$ & 9082.0 & $\begin{array}{l}14784 . \\
3\end{array}$ & $\begin{array}{l}15399 . \\
7\end{array}$ & $\begin{array}{l}13289 . \\
2\end{array}$ & $\begin{array}{l}11641 . \\
4\end{array}$ & 0.0 & 0.0 & 0.0 & 0.0 & 0.0 & 0.0 \\
\hline Margaroyl-EA & 313.3 & 9.4 & $\mathrm{C} 19 \mathrm{H} 39 \mathrm{NO} 2$ & $\begin{array}{l}\text { Fatty } \\
\text { Acyls }\end{array}$ & $\begin{array}{l}\text { Fatty } \\
\text { amides }\end{array}$ & $\begin{array}{l}11019 \\
.3\end{array}$ & 9032.2 & $\begin{array}{l}66291 . \\
1\end{array}$ & $\begin{array}{l}27098 . \\
3\end{array}$ & $\begin{array}{l}14854 . \\
7\end{array}$ & $\begin{array}{l}953023 \\
.2\end{array}$ & 0.0 & 0.0 & 0.0 & 0.0 & 0.0 & 0.0 \\
\hline Mayolene-18 & 560.5 & 7.0 & $\mathrm{C} 36 \mathrm{H} 64 \mathrm{O} 4$ & $\begin{array}{l}\text { Fatty } \\
\text { Acyls }\end{array}$ & $\begin{array}{l}\text { Fatty } \\
\text { esters }\end{array}$ & $\begin{array}{l}25574 \\
.8\end{array}$ & $\begin{array}{l}17279 . \\
2\end{array}$ & $\begin{array}{l}95986 . \\
6\end{array}$ & $\begin{array}{l}106021 \\
.2\end{array}$ & $\begin{array}{l}176803 \\
.6\end{array}$ & $\begin{array}{l}26414 . \\
4\end{array}$ & 0.0 & 0.0 & 0.0 & 0.0 & 0.0 & 0.0 \\
\hline $\operatorname{MG}(0: 0 / 18: 1(11 Z) / 0: 0)$ & 356.3 & 6.4 & $\mathrm{C} 21 \mathrm{H} 40 \mathrm{O} 4$ & $\begin{array}{l}\text { Glyceroli } \\
\text { pids }\end{array}$ & $\begin{array}{l}\text { Monoradyl } \\
\text { glycerols }\end{array}$ & $\begin{array}{l}23006 \\
.9\end{array}$ & $\begin{array}{l}19884 . \\
2\end{array}$ & $\begin{array}{l}29593 . \\
0\end{array}$ & $\begin{array}{l}58429 . \\
1\end{array}$ & $\begin{array}{l}61520 . \\
7\end{array}$ & $\begin{array}{l}30331 . \\
7\end{array}$ & 0.0 & 0.0 & 0.0 & 0.0 & 0.0 & 0.0 \\
\hline $\operatorname{MG}(0: 0 / 20: 1(11 Z) / 0: 0)$ & 401.4 & 4.7 & $\mathrm{C} 23 \mathrm{H} 44 \mathrm{O} 4$ & $\begin{array}{l}\text { Glyceroli } \\
\text { pids }\end{array}$ & $\begin{array}{l}\text { Monoradyl } \\
\text { glycerols }\end{array}$ & $\begin{array}{l}19334 \\
.8\end{array}$ & $\begin{array}{l}21599 . \\
8\end{array}$ & $\begin{array}{l}16366 . \\
6\end{array}$ & $\begin{array}{l}90513 . \\
1\end{array}$ & $\begin{array}{l}102930 \\
.9\end{array}$ & $\begin{array}{l}26665 . \\
9\end{array}$ & 0.0 & 0.0 & 0.0 & 0.0 & 0.0 & 0.0 \\
\hline $\operatorname{MG}(0: 0 / 22: 1(13 Z) / 0: 0)$ & 429.4 & 6.1 & $\mathrm{C} 25 \mathrm{H} 48 \mathrm{O} 4$ & $\begin{array}{l}\text { Glyceroli } \\
\text { pids }\end{array}$ & $\begin{array}{l}\text { Monoradyl } \\
\text { glycerols }\end{array}$ & $\begin{array}{l}22202 \\
.7\end{array}$ & $\begin{array}{l}23249 . \\
9\end{array}$ & $\begin{array}{l}34375 . \\
5\end{array}$ & $\begin{array}{l}284545 \\
.0\end{array}$ & $\begin{array}{l}370697 \\
.9\end{array}$ & $\begin{array}{l}45596 . \\
4\end{array}$ & $\begin{array}{l}72187 . \\
6\end{array}$ & $\begin{array}{l}26653 . \\
4\end{array}$ & $\begin{array}{l}27173 . \\
8\end{array}$ & $\begin{array}{l}39076 . \\
8\end{array}$ & $\begin{array}{l}50983 . \\
2\end{array}$ & $\begin{array}{l}34197 . \\
2\end{array}$ \\
\hline
\end{tabular}




\begin{tabular}{|c|c|c|c|c|c|c|c|c|c|c|c|c|c|c|c|c|c|}
\hline $\operatorname{MG}(0: 0 / 24: 1(15 Z) / 0: 0)$ & 440.4 & 11.3 & $\mathrm{C} 27 \mathrm{H} 52 \mathrm{O} 4$ & $\begin{array}{l}\text { Glyceroli } \\
\text { pids }\end{array}$ & $\begin{array}{l}\text { Monoradyl } \\
\text { glycerols }\end{array}$ & $\begin{array}{l}36063 \\
.6\end{array}$ & $\begin{array}{l}30374 . \\
0\end{array}$ & $\begin{array}{l}52251 . \\
4\end{array}$ & $\begin{array}{l}44385 . \\
0\end{array}$ & $\begin{array}{l}54397 . \\
1\end{array}$ & $\begin{array}{l}51090 . \\
8\end{array}$ & 0.0 & 0.0 & 0.0 & 0.0 & 0.0 & 0.0 \\
\hline $\mathrm{MG}(18: 0 / 0: 0 / 0: 0)$ & 380.3 & 7.4 & $\mathrm{C} 21 \mathrm{H} 42 \mathrm{O} 4$ & $\begin{array}{l}\text { Glyceroli } \\
\text { pids }\end{array}$ & $\begin{array}{l}\text { Monoradyl } \\
\text { glycerols }\end{array}$ & 0.0 & 0.0 & 0.0 & 0.0 & 0.0 & 0.0 & $\begin{array}{l}922532 \\
.3\end{array}$ & $\begin{array}{l}835708 \\
.4\end{array}$ & $\begin{array}{l}832124 \\
.7\end{array}$ & $\begin{array}{l}780067 \\
.9\end{array}$ & $\begin{array}{l}675920 \\
.8\end{array}$ & $\begin{array}{l}636039 \\
.5\end{array}$ \\
\hline MG(20:3) & 380.3 & 6.2 & $\mathrm{C} 23 \mathrm{H} 40 \mathrm{O} 4$ & $\begin{array}{l}\text { Glyceroli } \\
\text { pids }\end{array}$ & $\begin{array}{l}\text { Monoradyl } \\
\text { glycerols }\end{array}$ & $\begin{array}{l}19877 \\
.7\end{array}$ & $\begin{array}{l}23035 . \\
1\end{array}$ & $\begin{array}{l}25699 . \\
0\end{array}$ & $\begin{array}{l}36356 . \\
2\end{array}$ & $\begin{array}{l}41853 . \\
5\end{array}$ & $\begin{array}{l}29765 . \\
3\end{array}$ & $\begin{array}{l}30075 . \\
9\end{array}$ & $\begin{array}{l}24318 \\
8\end{array}$ & $\begin{array}{l}26915 . \\
7\end{array}$ & $\begin{array}{l}25712 . \\
5\end{array}$ & $\begin{array}{l}19903 . \\
3\end{array}$ & $\begin{array}{l}22153 . \\
9\end{array}$ \\
\hline $\operatorname{MGDG}\left(16: 0 / 18: 2\left(9 Z \_12 Z\right)\right)$ & 800.6 & 8.8 & $\mathrm{C} 43 \mathrm{H} 78 \mathrm{O} 10$ & $\begin{array}{l}\text { Glyceroli } \\
\text { pids }\end{array}$ & $\begin{array}{l}\text { Glycosyldi } \\
\text { radylglyce } \\
\text { rols }\end{array}$ & $\begin{array}{l}40723 \\
.3\end{array}$ & $\begin{array}{l}65127 . \\
3\end{array}$ & $\begin{array}{l}76394 . \\
3\end{array}$ & $\begin{array}{l}117275 \\
.1\end{array}$ & $\begin{array}{l}111829 \\
.7\end{array}$ & $\begin{array}{l}177991 \\
.5\end{array}$ & 0.0 & 0.0 & 0.0 & 0.0 & 0.0 & 0.0 \\
\hline Mycolipanolic acid (C28) & 440.4 & 11.1 & $\mathrm{C} 28 \mathrm{H} 56 \mathrm{O} 3$ & $\begin{array}{l}\text { Fatty } \\
\text { Acyls }\end{array}$ & $\begin{array}{l}\text { Fatty } \\
\text { Acids and } \\
\text { Conjugates }\end{array}$ & $\begin{array}{l}24013 \\
.3\end{array}$ & $\begin{array}{l}18006 . \\
8\end{array}$ & $\begin{array}{l}28017 . \\
7\end{array}$ & $\begin{array}{l}27917 . \\
3\end{array}$ & $\begin{array}{l}21986 . \\
8\end{array}$ & $\begin{array}{l}19833 . \\
1\end{array}$ & 0.0 & 0.0 & 0.0 & 0.0 & 0.0 & 0.0 \\
\hline $\begin{array}{l}\mathrm{N} \text {-(2-hydroxy-15Z- } \\
\text { tetracosenoyl)-4E_6E- } \\
\text { hexadecasphingadienine-1- } \\
\text { phosphoethanolamine }\end{array}$ & 802.6 & 9.4 & $\begin{array}{l}\mathrm{C} 42 \mathrm{H} 81 \mathrm{~N} 2 \mathrm{O} 7 \\
\mathrm{P}\end{array}$ & $\begin{array}{l}\text { Sphingoli } \\
\text { pids }\end{array}$ & $\begin{array}{l}\text { Ceramide } \\
\text { phosphoet } \\
\text { hanolamin } \\
\text { es } \\
\text { [SP0302] }\end{array}$ & $\begin{array}{l}38988 \\
.9\end{array}$ & $\begin{array}{l}59100 . \\
6\end{array}$ & $\begin{array}{l}59955 . \\
5\end{array}$ & $\begin{array}{l}65608 \\
6\end{array}$ & $\begin{array}{l}40665 . \\
6\end{array}$ & $\begin{array}{l}137377 \\
.8\end{array}$ & 0.0 & 0.0 & 0.0 & 0.0 & 0.0 & 0.0 \\
\hline $\begin{array}{l}\mathrm{N}-(2 \text {-hydroxy-docosanoyl)-1- } \\
\text { beta-glucosyl- } \\
\text { tetradecasphing-4-enine }\end{array}$ & 779.6 & 14.3 & $\mathrm{C} 42 \mathrm{H} 81 \mathrm{NO} 9$ & $\begin{array}{l}\text { Sphingoli } \\
\text { pids }\end{array}$ & $\begin{array}{l}\text { Ceramide } \\
\text { phosphoet } \\
\text { hanolamin } \\
\text { es } \\
\text { [SP0302] }\end{array}$ & $\begin{array}{l}22406 \\
.8\end{array}$ & $\begin{array}{l}12909 . \\
4\end{array}$ & $\begin{array}{l}10405 . \\
4\end{array}$ & 7308.2 & $\begin{array}{l}20208 . \\
4\end{array}$ & $\begin{array}{l}20232 . \\
0\end{array}$ & 8937.3 & 9531.6 & 7099.5 & 7762.4 & 8526.2 & 8421.6 \\
\hline $\begin{array}{l}\text { N-(2-hydroxy-docosanoyl)- } \\
\text { tetradecasphing-4-enine-1- } \\
\text { phosphoethanolamine }\end{array}$ & 750.6 & 9.1 & $\begin{array}{l}\text { C38H77N2O7 } \\
P\end{array}$ & $\begin{array}{l}\text { Sphingoli } \\
\text { pids }\end{array}$ & $\begin{array}{l}\text { Ceramide } \\
\text { phosphoet } \\
\text { hanolamin } \\
\text { es } \\
\text { [SP0302] }\end{array}$ & $\begin{array}{l}6843 . \\
5\end{array}$ & 0.0 & 5525.5 & 0.0 & 7970.5 & 0.0 & 0.0 & 0.0 & 0.0 & 0.0 & 0.0 & 0.0 \\
\hline $\begin{array}{l}\text { N-(2-hydroxy-eicosanoyl)- } \\
\text { pentadecasphing-4-enine-1- } \\
\text { phosphoethanolamine }\end{array}$ & 690.5 & 10.5 & $\begin{array}{l}\mathrm{C} 37 \mathrm{H} 75 \mathrm{~N} 2 \mathrm{O} 7 \\
\mathrm{P}\end{array}$ & $\begin{array}{l}\text { Sphingoli } \\
\text { pids }\end{array}$ & $\begin{array}{l}\text { Ceramide } \\
\text { phosphoet } \\
\text { hanolamin } \\
\text { es } \\
\text { [SP0302] }\end{array}$ & $\begin{array}{l}39471 \\
.5\end{array}$ & $\begin{array}{l}41020 . \\
5\end{array}$ & $\begin{array}{l}40313 . \\
7\end{array}$ & $\begin{array}{l}39030 . \\
8\end{array}$ & $\begin{array}{l}42436 . \\
6\end{array}$ & $\begin{array}{l}37807 . \\
7\end{array}$ & 0.0 & 0.0 & 0.0 & 0.0 & 0.0 & 0.0 \\
\hline $\begin{array}{l}\mathrm{N} \text {-(2-hydroxy-hexacosanoyl)- } \\
\text { tetradecasphing-4-enine }\end{array}$ & 637.6 & 15.2 & $\mathrm{C} 40 \mathrm{H} 79 \mathrm{NO} 4$ & $\begin{array}{l}\text { Sphingoli } \\
\text { pids }\end{array}$ & $\begin{array}{l}\mathrm{N}- \\
\text { acylsphing } \\
\text { osines } \\
\text { (ceramides } \\
\text { ) [SP0201] }\end{array}$ & $\begin{array}{l}36751 \\
.1\end{array}$ & $\begin{array}{l}59429 . \\
7\end{array}$ & $\begin{array}{l}85820 . \\
1\end{array}$ & $\begin{array}{l}121414 \\
.1\end{array}$ & $\begin{array}{l}62880 . \\
3\end{array}$ & $\begin{array}{l}97684 . \\
4\end{array}$ & 7496.2 & 6062.7 & 4697.1 & 7021.1 & 0.0 & 7208.4 \\
\hline
\end{tabular}




\begin{tabular}{|c|c|c|c|c|c|c|c|c|c|c|c|c|c|c|c|c|c|}
\hline $\begin{array}{l}\mathrm{N} \text {-(2-hydroxy-octadecanoyl)- } \\
\text { tetradecasphing-4-enine-1- } \\
\text { phosphoethanolamine }\end{array}$ & 648.5 & 8.7 & $\begin{array}{l}\mathrm{C} 34 \mathrm{H} 69 \mathrm{~N} 2 \mathrm{O} 7 \\
\mathrm{P}\end{array}$ & $\begin{array}{l}\text { Sphingoli } \\
\text { pids }\end{array}$ & $\begin{array}{l}\text { Ceramide } \\
\text { phosphoet } \\
\text { hanolamin } \\
\text { es } \\
\text { [SP0302] }\end{array}$ & $\begin{array}{l}34787 \\
.7\end{array}$ & $\begin{array}{l}17663 . \\
0\end{array}$ & $\begin{array}{l}70336 . \\
5\end{array}$ & $\begin{array}{l}91061 . \\
2\end{array}$ & $\begin{array}{l}122207 \\
.0\end{array}$ & $\begin{array}{l}56952 . \\
0\end{array}$ & $\begin{array}{l}41484 . \\
4\end{array}$ & $\begin{array}{l}40365 \\
2\end{array}$ & $\begin{array}{l}29739 . \\
1\end{array}$ & $\begin{array}{l}24716 . \\
2\end{array}$ & $\begin{array}{l}29247 . \\
5\end{array}$ & $\begin{array}{l}13612 . \\
1\end{array}$ \\
\hline $\begin{array}{l}\text { N-(2-hydroxy-tetracosanoyl)- } \\
\text { hexadecasphing-4-enine-1- } \\
\text { phosphoethanolamine }\end{array}$ & 760.6 & 11.8 & $\begin{array}{l}\mathrm{C} 42 \mathrm{H} 85 \mathrm{~N} 2 \mathrm{O} 7 \\
\mathrm{P}\end{array}$ & $\begin{array}{l}\text { Sphingoli } \\
\text { pids }\end{array}$ & $\begin{array}{l}\text { Ceramide } \\
\text { phosphoet } \\
\text { hanolamin } \\
\text { es } \\
\text { [SP0302] }\end{array}$ & $\begin{array}{l}54844 \\
.9\end{array}$ & $\begin{array}{l}74190 . \\
3\end{array}$ & $\begin{array}{l}77638 \\
2\end{array}$ & $\begin{array}{l}98743 . \\
6\end{array}$ & $\begin{array}{l}126138 \\
.7\end{array}$ & $\begin{array}{l}95883 . \\
4\end{array}$ & 0.0 & 0.0 & 0.0 & 0.0 & 0.0 & 0.0 \\
\hline $\begin{array}{l}\text { N-(2-hydroxydocosanoyl)- } \\
\text { 4R-hydroxysphinganine-1- } \\
\text { phospho-(1'-myo-inositol) }\end{array}$ & 897.6 & 11.2 & $\begin{array}{l}\mathrm{C} 46 \mathrm{H} 92 \mathrm{NO} 13 \\
\mathrm{P}\end{array}$ & $\begin{array}{l}\text { Sphingoli } \\
\text { pids }\end{array}$ & $\begin{array}{l}\text { Ceramide } \\
\text { phosphoin } \\
\text { ositols } \\
\text { [SP0303] }\end{array}$ & $\begin{array}{l}46869 \\
.1\end{array}$ & $\begin{array}{l}75939 . \\
6\end{array}$ & $\begin{array}{l}105270 \\
.7\end{array}$ & $\begin{array}{l}106538 \\
.6\end{array}$ & $\begin{array}{l}172157 \\
.0\end{array}$ & $\begin{array}{l}106484 \\
.9\end{array}$ & 0.0 & 0.0 & 0.0 & 0.0 & 0.0 & 0.0 \\
\hline $\begin{array}{l}\text { N-(2-hydroxyhexacosanoyl)- } \\
\text { eicosasphinganine }\end{array}$ & 723.7 & 21.7 & $\mathrm{C} 46 \mathrm{H} 93 \mathrm{NO} 4$ & $\begin{array}{l}\text { Sphingoli } \\
\text { pids }\end{array}$ & $\begin{array}{l}\text { Ceramide } \\
\text { phosphoin } \\
\text { ositols } \\
\text { [SP0303] }\end{array}$ & $\begin{array}{l}32197 \\
.3\end{array}$ & $\begin{array}{l}24254 \\
5\end{array}$ & $\begin{array}{l}20915 . \\
8\end{array}$ & $\begin{array}{l}35951 . \\
2\end{array}$ & $\begin{array}{l}35097 \\
7\end{array}$ & $\begin{array}{l}29881 . \\
9\end{array}$ & 0.0 & 0.0 & 0.0 & 0.0 & 0.0 & 0.0 \\
\hline $\begin{array}{l}\text { N-(2-hydroxytetracosanoyl)- } \\
\text { 4R-hydroxysphinganine-1- } \\
\text { phospho-(1'-myo-inositol) }\end{array}$ & 925.7 & 12.3 & $\begin{array}{l}\mathrm{C} 48 \mathrm{H} 96 \mathrm{NO} 13 \\
\mathrm{P}\end{array}$ & $\begin{array}{l}\text { Sphingoli } \\
\text { pids }\end{array}$ & $\begin{array}{l}\text { Ceramide } \\
\text { phosphoin } \\
\text { ositols } \\
\text { [SP0303] }\end{array}$ & $\begin{array}{l}12368 \\
6.6\end{array}$ & $\begin{array}{l}187263 \\
.2\end{array}$ & $\begin{array}{l}253769 \\
.2\end{array}$ & $\begin{array}{l}266206 \\
.1\end{array}$ & $\begin{array}{l}330297 \\
.4\end{array}$ & $\begin{array}{l}219250 \\
.5\end{array}$ & 0.0 & 0.0 & 0.0 & 0.0 & 0.0 & 0.0 \\
\hline $\begin{array}{l}\text { N-(5-hydroxy-pentyl)- } \\
5 Z \text { _Z__11Z_14Z- } \\
\text { eicosatetraenoyl amine }\end{array}$ & 389.3 & 4.9 & $\mathrm{C} 25 \mathrm{H} 43 \mathrm{NO} 2$ & $\begin{array}{l}\text { Sphingoli } \\
\text { pids }\end{array}$ & $\begin{array}{l}\text { Ceramide } \\
\text { phosphoin } \\
\text { ositols } \\
\text { [SP0303] }\end{array}$ & $\begin{array}{l}3254 . \\
2\end{array}$ & 0.0 & 0.0 & 2454.2 & 3275.0 & 2424.8 & 2630.9 & 0.0 & 0.0 & 0.0 & 0.0 & 0.0 \\
\hline $\begin{array}{l}\mathrm{N} \text {-(9Z-octadecenoyl)-4E_6E- } \\
\text { tetradecasphingadienine-1- } \\
\text { phosphoethanolamine }\end{array}$ & 628.5 & 7.4 & $\begin{array}{l}\mathrm{C} 34 \mathrm{H} 65 \mathrm{~N} 2 \mathrm{O} 6 \\
\mathrm{P}\end{array}$ & $\begin{array}{l}\text { Sphingoli } \\
\text { pids }\end{array}$ & $\begin{array}{l}\text { Ceramide } \\
\text { phosphoet } \\
\text { hanolamin } \\
\text { es } \\
\text { [SP0302] }\end{array}$ & $\begin{array}{l}8436 . \\
5\end{array}$ & $\begin{array}{l}14778 . \\
8\end{array}$ & $\begin{array}{l}33943 . \\
2\end{array}$ & $\begin{array}{l}36400 . \\
8\end{array}$ & $\begin{array}{l}43525 . \\
3\end{array}$ & $\begin{array}{l}16270 . \\
8\end{array}$ & 0.0 & 0.0 & 0.0 & 0.0 & 0.0 & 0.0 \\
\hline $\begin{array}{l}\text { N-(acetyl)-sphing-4-enine-1- } \\
\text { phosphate }\end{array}$ & 421.3 & 1.7 & $\mathrm{C} 20 \mathrm{H} 40 \mathrm{NO} 6 \mathrm{P}$ & $\begin{array}{l}\text { Sphingoli } \\
\text { pids }\end{array}$ & $\begin{array}{l}\text { Ceramide } \\
1- \\
\text { phosphates } \\
\text { [SP0205] }\end{array}$ & $\begin{array}{l}33114 \\
50.8\end{array}$ & $\begin{array}{l}143909 \\
8.5\end{array}$ & $\begin{array}{l}147571 \\
04.0\end{array}$ & $\begin{array}{l}101743 \\
62.0\end{array}$ & $\begin{array}{l}449065 \\
16.0\end{array}$ & $\begin{array}{l}515098 \\
2.5\end{array}$ & $\begin{array}{l}141185 \\
3.0\end{array}$ & $\begin{array}{l}111901 \\
4.6\end{array}$ & $\begin{array}{l}141398 \\
2.6\end{array}$ & $\begin{array}{l}120847 \\
3.9\end{array}$ & $\begin{array}{l}773074 \\
.2\end{array}$ & $\begin{array}{l}878788 \\
.1\end{array}$ \\
\hline $\begin{array}{l}\text { N-(docosanoyl)-4R- } \\
\text { hydroxysphinganine-1- } \\
\text { phospho-(1'-myo-inositol) }\end{array}$ & 881.6 & 11.6 & $\begin{array}{l}\mathrm{C} 46 \mathrm{H} 92 \mathrm{NO} 12 \\
\mathrm{P}\end{array}$ & $\begin{array}{l}\text { Sphingoli } \\
\text { pids }\end{array}$ & $\begin{array}{l}\text { Ceramide } \\
\text { phosphoin } \\
\text { ositols } \\
\text { [SP0303] }\end{array}$ & $\begin{array}{l}20279 \\
.2\end{array}$ & $\begin{array}{l}41632 . \\
6\end{array}$ & $\begin{array}{l}62918 . \\
4\end{array}$ & $\begin{array}{l}70660 . \\
5\end{array}$ & $\begin{array}{l}114647 \\
.9\end{array}$ & $\begin{array}{l}47453 . \\
9\end{array}$ & 0.0 & 0.0 & 0.0 & 0.0 & 0.0 & 0.0 \\
\hline
\end{tabular}




\begin{tabular}{|c|c|c|c|c|c|c|c|c|c|c|c|c|c|c|c|c|c|}
\hline $\begin{array}{l}\mathrm{N}-(\text { docosanoyl)-sphinganine- } \\
\text { 1-phospho-(1'-myo-inositol) }\end{array}$ & 911.6 & 11.8 & $\begin{array}{l}\mathrm{C} 46 \mathrm{H} 92 \mathrm{NO} 11 \\
\mathrm{P}\end{array}$ & $\begin{array}{l}\text { Sphingoli } \\
\text { pids }\end{array}$ & $\begin{array}{l}\text { Ceramide } \\
\text { phosphoin } \\
\text { ositols } \\
\text { [SP0303] }\end{array}$ & $\begin{array}{l}18415 \\
3.0\end{array}$ & $\begin{array}{l}277876 \\
.3\end{array}$ & $\begin{array}{l}406856 \\
.2\end{array}$ & $\begin{array}{l}479349 \\
.2\end{array}$ & $\begin{array}{l}625090 \\
.6\end{array}$ & $\begin{array}{l}387110 \\
.0\end{array}$ & 0.0 & 0.0 & 0.0 & 0.0 & 0.0 & 0.0 \\
\hline $\begin{array}{l}\mathrm{N} \text {-(hexadecanoyl)- } \\
\text { tetradecasphing-4-enine-1- } \\
\text { phosphoethanolamine }\end{array}$ & 604.5 & 7.6 & $\begin{array}{l}\mathrm{C} 32 \mathrm{H} 65 \mathrm{~N} 2 \mathrm{O} 6 \\
\mathrm{P}\end{array}$ & $\begin{array}{l}\text { Sphingoli } \\
\text { pids }\end{array}$ & $\begin{array}{l}\text { Ceramide } \\
\text { phosphoet } \\
\text { hanolamin } \\
\text { es } \\
\text { [SP0302] }\end{array}$ & $\begin{array}{l}9768 . \\
0\end{array}$ & 9609.3 & $\begin{array}{l}20259 . \\
3\end{array}$ & $\begin{array}{l}23812 . \\
1\end{array}$ & $\begin{array}{l}26160 . \\
7\end{array}$ & $\begin{array}{l}12650 . \\
8\end{array}$ & 0.0 & 0.0 & 0.0 & 0.0 & 0.0 & 0.0 \\
\hline $\begin{array}{l}\text { N-Methylethanolamine } \\
\text { phosphate }\end{array}$ & 155.0 & 1.3 & C3H10NO4P & $\begin{array}{l}\text { Lipid } \\
\text { Metabolis } \\
\mathrm{m}\end{array}$ & $\begin{array}{l}\text { Glyceroph } \\
\text { ospholipid } \\
\text { metabolis } \\
\text { m }\end{array}$ & 0.0 & 2919.5 & 0.0 & 0.0 & 0.0 & 4622.7 & 0.0 & 0.0 & 0.0 & 0.0 & 0.0 & 0.0 \\
\hline N-palmitoyl proline & 370.3 & 3.1 & $\mathrm{C} 21 \mathrm{H} 39 \mathrm{NO} 3$ & $\begin{array}{l}\text { Fatty } \\
\text { Acyls }\end{array}$ & $\begin{array}{l}\text { Fatty } \\
\text { amides }\end{array}$ & $\begin{array}{l}42163 \\
.4\end{array}$ & $\begin{array}{l}21099 \\
6\end{array}$ & $\begin{array}{l}33408 . \\
9\end{array}$ & $\begin{array}{l}50839 . \\
6\end{array}$ & $\begin{array}{l}28783 \\
0\end{array}$ & $\begin{array}{l}160599 \\
.6\end{array}$ & $\begin{array}{l}41734 . \\
8\end{array}$ & $\begin{array}{l}61944 . \\
1\end{array}$ & $\begin{array}{l}40008 \\
1\end{array}$ & $\begin{array}{l}47093 . \\
0\end{array}$ & $\begin{array}{l}40772 . \\
0\end{array}$ & $\begin{array}{l}93975 . \\
9\end{array}$ \\
\hline N-stearoyl tryptophan & 492.3 & 1.8 & $\mathrm{C} 29 \mathrm{H} 46 \mathrm{~N} 2 \mathrm{O} 3$ & $\begin{array}{l}\text { Fatty } \\
\text { Acyls }\end{array}$ & $\begin{array}{l}\text { Fatty } \\
\text { amides }\end{array}$ & $\begin{array}{l}7537 . \\
7\end{array}$ & 8993.9 & $\begin{array}{l}21762 . \\
9\end{array}$ & $\begin{array}{l}14245 . \\
1\end{array}$ & $\begin{array}{l}32262 . \\
0\end{array}$ & $\begin{array}{l}11413 . \\
7\end{array}$ & 0.0 & 0.0 & 0.0 & 0.0 & 0.0 & 0.0 \\
\hline $\mathrm{N}$-stearoyl tyrosine & 447.3 & 4.8 & $\mathrm{C} 27 \mathrm{H} 45 \mathrm{NO} 4$ & $\begin{array}{l}\text { Fatty } \\
\text { Acyls }\end{array}$ & $\begin{array}{l}\text { Fatty } \\
\text { amides }\end{array}$ & $\begin{array}{l}47422 \\
.3\end{array}$ & $\begin{array}{l}84361 . \\
2\end{array}$ & $\begin{array}{l}94263 . \\
5\end{array}$ & $\begin{array}{l}92353 . \\
8\end{array}$ & $\begin{array}{l}73882 . \\
6\end{array}$ & $\begin{array}{l}205442 \\
.2\end{array}$ & 0.0 & 0.0 & 0.0 & 0.0 & 0.0 & 0.0 \\
\hline Noladin Ether & 364.3 & 8.6 & $\mathrm{C} 23 \mathrm{H} 40 \mathrm{O} 3$ & $\begin{array}{l}\text { Glyceroli } \\
\text { pids }\end{array}$ & $\begin{array}{l}\text { Monoradyl } \\
\text { glycerols }\end{array}$ & $\begin{array}{l}8506 . \\
4\end{array}$ & $\begin{array}{l}11957 . \\
2\end{array}$ & $\begin{array}{l}14993 . \\
0\end{array}$ & $\begin{array}{l}14877 . \\
5\end{array}$ & $\begin{array}{l}16487 . \\
9\end{array}$ & $\begin{array}{l}10969 . \\
7\end{array}$ & $\begin{array}{l}107599 \\
.9\end{array}$ & $\begin{array}{l}96597 . \\
9\end{array}$ & $\begin{array}{l}104857 \\
.3\end{array}$ & $\begin{array}{l}121722 \\
.7\end{array}$ & $\begin{array}{l}94755 . \\
9\end{array}$ & $\begin{array}{l}82094 . \\
9\end{array}$ \\
\hline O-Butanoylcarnitine & 231.1 & 1.4 & $\mathrm{C} 11 \mathrm{H} 21 \mathrm{NO} 4$ & $\begin{array}{l}\text { Fatty } \\
\text { Acyls }\end{array}$ & $\begin{array}{l}\text { Fatty acyl } \\
\text { carnitines }\end{array}$ & 0.0 & $\begin{array}{l}10806 . \\
7\end{array}$ & 6754.8 & $\begin{array}{l}29025 . \\
4\end{array}$ & 0.0 & $\begin{array}{l}51794 . \\
0\end{array}$ & 0.0 & 0.0 & 0.0 & 0.0 & 0.0 & 0.0 \\
\hline O-Propanoylcarnitine & 217.1 & 1.3 & $\mathrm{C} 10 \mathrm{H} 19 \mathrm{NO} 4$ & $\begin{array}{l}\text { Fatty } \\
\text { Acyls }\end{array}$ & $\begin{array}{l}\text { Oxidationo } \\
\text { fBranched } \\
\text { FattyAcids }\end{array}$ & $\begin{array}{l}26630 \\
.6\end{array}$ & $\begin{array}{l}80046 . \\
5\end{array}$ & $\begin{array}{l}158717 \\
.7\end{array}$ & $\begin{array}{l}376527 \\
.0\end{array}$ & $\begin{array}{l}32451 . \\
4\end{array}$ & $\begin{array}{l}472535 \\
.5\end{array}$ & 0.0 & 0.0 & 0.0 & 0.0 & 0.0 & 0.0 \\
\hline Octadecanamide & 283.3 & 7.6 & $\mathrm{C} 18 \mathrm{H} 37 \mathrm{NO}$ & $\begin{array}{l}\text { Fatty } \\
\text { Acyls }\end{array}$ & $\begin{array}{l}\text { Fatty } \\
\text { amides }\end{array}$ & $\begin{array}{l}33920 \\
7.8\end{array}$ & $\begin{array}{l}211972 \\
.2\end{array}$ & $\begin{array}{l}445885 \\
.6\end{array}$ & $\begin{array}{l}611348 \\
.0\end{array}$ & $\begin{array}{l}463333 \\
.8\end{array}$ & $\begin{array}{l}452180 \\
.0\end{array}$ & $\begin{array}{l}396527 \\
.5\end{array}$ & $\begin{array}{l}329922 \\
.4\end{array}$ & $\begin{array}{l}332363 \\
.8\end{array}$ & $\begin{array}{l}341905 \\
.8\end{array}$ & $\begin{array}{l}187579 \\
5.5\end{array}$ & $\begin{array}{l}318178 \\
.4\end{array}$ \\
\hline Octadecanoic acid & 284.3 & 8.7 & $\mathrm{C} 18 \mathrm{H} 36 \mathrm{O} 2$ & $\begin{array}{l}\text { Lipid } \\
\text { Metabolis } \\
\mathrm{m}\end{array}$ & $\begin{array}{l}\text { Fatty acid } \\
\text { biosynthes } \\
\text { is_Biosyn } \\
\text { thesis of } \\
\text { unsaturate } \\
\mathrm{d} \text { fatty } \\
\text { acids }\end{array}$ & $\begin{array}{l}78566 \\
33.0\end{array}$ & $\begin{array}{l}534811 \\
4.0\end{array}$ & $\begin{array}{l}139521 \\
94.0\end{array}$ & $\begin{array}{l}178324 \\
40.0\end{array}$ & $\begin{array}{l}256029 \\
74.0\end{array}$ & $\begin{array}{l}128556 \\
51.0\end{array}$ & $\begin{array}{l}100363 \\
86.0\end{array}$ & $\begin{array}{l}100131 \\
38.0\end{array}$ & $\begin{array}{l}885121 \\
0.0\end{array}$ & $\begin{array}{l}861458 \\
7.0\end{array}$ & $\begin{array}{l}139792 \\
03.0\end{array}$ & $\begin{array}{l}630464 \\
9.0\end{array}$ \\
\hline
\end{tabular}




\begin{tabular}{|c|c|c|c|c|c|c|c|c|c|c|c|c|c|c|c|c|c|}
\hline $\mathrm{PA}(20: 0 / 0: 0)$ & 466.3 & 9.1 & $\mathrm{C} 23 \mathrm{H} 47 \mathrm{O} 7 \mathrm{P}$ & $\begin{array}{l}\text { Glyceroph } \\
\text { ospholipid } \\
\text { s }\end{array}$ & $\begin{array}{l}\text { Glyceroph } \\
\text { osphates }\end{array}$ & $\begin{array}{l}11278 \\
.4\end{array}$ & 5241.9 & $\begin{array}{l}37131 . \\
7\end{array}$ & $\begin{array}{l}25970 . \\
6\end{array}$ & $\begin{array}{l}24401 . \\
7\end{array}$ & $\begin{array}{l}27461 . \\
2\end{array}$ & $\begin{array}{l}10979 . \\
5\end{array}$ & 7739.7 & $\begin{array}{l}17327 . \\
9\end{array}$ & $\begin{array}{l}10330 . \\
5\end{array}$ & $\begin{array}{l}10724 . \\
9\end{array}$ & 9632.7 \\
\hline PA(30:4) & 629.4 & 7.0 & C33H57O8P & $\begin{array}{l}\text { Glyceroph } \\
\text { ospholipid } \\
\text { s }\end{array}$ & $\begin{array}{l}\text { Glyceroph } \\
\text { osphates }\end{array}$ & $\begin{array}{l}11035 \\
.2\end{array}$ & 2951.2 & 0.0 & 0.0 & 0.0 & 0.0 & 0.0 & 0.0 & 0.0 & 0.0 & 0.0 & 0.0 \\
\hline $\mathrm{PA}(31: 0)$ & 634.5 & 15.8 & C34H67O8P & $\begin{array}{l}\text { Glyceroph } \\
\text { ospholipid } \\
\text { s }\end{array}$ & $\begin{array}{l}\text { Glyceroph } \\
\text { osphates }\end{array}$ & 0.0 & 0.0 & 0.0 & 0.0 & 9644.5 & 0.0 & 0.0 & 0.0 & 0.0 & 0.0 & 0.0 & 0.0 \\
\hline PA(33:1) & 660.5 & 13.3 & C36H69O8P & $\begin{array}{l}\text { Glyceroph } \\
\text { ospholipid } \\
\text { s }\end{array}$ & $\begin{array}{l}\text { Glyceroph } \\
\text { osphates }\end{array}$ & $\begin{array}{l}24752 \\
.2\end{array}$ & 0.0 & 0.0 & 0.0 & 0.0 & 0.0 & 0.0 & 0.0 & 0.0 & 0.0 & 0.0 & 0.0 \\
\hline PA(34:1) & 674.5 & 17.0 & $\mathrm{C} 37 \mathrm{H} 71 \mathrm{O} 8 \mathrm{P}$ & $\begin{array}{l}\text { Glyceroph } \\
\text { ospholipid } \\
\text { s }\end{array}$ & $\begin{array}{l}\text { Glyceroph } \\
\text { osphates }\end{array}$ & 0.0 & 0.0 & 0.0 & $\begin{array}{l}10236 . \\
3\end{array}$ & 0.0 & 0.0 & 0.0 & 0.0 & 0.0 & 0.0 & 0.0 & 0.0 \\
\hline PA(35:0) & 690.5 & 14.9 & C38H75O8P & $\begin{array}{l}\text { Glyceroph } \\
\text { ospholipid } \\
\text { s }\end{array}$ & $\begin{array}{l}\text { Glyceroph } \\
\text { osphates }\end{array}$ & $\begin{array}{l}16305 \\
1.0\end{array}$ & $\begin{array}{l}285492 \\
.9\end{array}$ & $\begin{array}{l}310409 \\
.3\end{array}$ & $\begin{array}{l}455822 \\
.1\end{array}$ & $\begin{array}{l}70362 . \\
0\end{array}$ & $\begin{array}{l}243300 \\
.1\end{array}$ & 0.0 & 0.0 & 0.0 & 0.0 & 0.0 & 0.0 \\
\hline $\operatorname{PA}(35: 2)$ & 686.5 & 13.9 & C38H71O8P & $\begin{array}{l}\text { Glyceroph } \\
\text { ospholipid } \\
\text { s }\end{array}$ & $\begin{array}{l}\text { Glyceroph } \\
\text { osphates }\end{array}$ & $\begin{array}{l}15120 \\
.1\end{array}$ & $\begin{array}{l}27626 . \\
5\end{array}$ & $\begin{array}{l}14806 . \\
2\end{array}$ & $\begin{array}{l}25129 . \\
9\end{array}$ & $\begin{array}{l}17415 . \\
4\end{array}$ & $\begin{array}{l}42134 . \\
4\end{array}$ & 0.0 & 0.0 & 0.0 & 0.0 & 0.0 & 0.0 \\
\hline $\operatorname{PA}(35: 3)$ & 684.5 & 13.3 & С38H69O8P & $\begin{array}{l}\text { Glyceroph } \\
\text { ospholipid } \\
\text { s }\end{array}$ & $\begin{array}{l}\text { Glyceroph } \\
\text { osphates }\end{array}$ & $\begin{array}{l}21610 \\
.0\end{array}$ & $\begin{array}{l}34088 . \\
3\end{array}$ & $\begin{array}{l}23067 . \\
3\end{array}$ & $\begin{array}{l}27679 . \\
1\end{array}$ & $\begin{array}{l}28682 . \\
3\end{array}$ & $\begin{array}{l}46008 . \\
1\end{array}$ & 0.0 & 0.0 & 0.0 & 0.0 & 0.0 & 0.0 \\
\hline $\operatorname{PA}(36: 1)$ & 702.5 & 18.3 & $\mathrm{C} 39 \mathrm{H} 75 \mathrm{O} 8 \mathrm{P}$ & $\begin{array}{l}\text { Glyceroph } \\
\text { ospholipid } \\
\mathrm{s}\end{array}$ & $\begin{array}{l}\text { Glyceroph } \\
\text { osphates }\end{array}$ & $\begin{array}{l}7563 . \\
3\end{array}$ & 7127.1 & 0.0 & $\begin{array}{l}18945 . \\
7\end{array}$ & 7809.0 & 9884.0 & 0.0 & 0.0 & 0.0 & 0.0 & 0.0 & 0.0 \\
\hline $\mathrm{PA}(36: 2)$ & 700.5 & 17.5 & С $39 \mathrm{H} 73 \mathrm{O} 8 \mathrm{P}$ & $\begin{array}{l}\text { Glyceroph } \\
\text { ospholipid } \\
\mathrm{s}\end{array}$ & $\begin{array}{l}\text { Glyceroph } \\
\text { osphates }\end{array}$ & 0.0 & 0.0 & 0.0 & $\begin{array}{l}15363 . \\
8\end{array}$ & 0.0 & 0.0 & 0.0 & 0.0 & 0.0 & 0.0 & 0.0 & 0.0 \\
\hline $\operatorname{PA}(37: 1)$ & 716.5 & 18.9 & $\mathrm{C} 40 \mathrm{H} 77 \mathrm{O} 8 \mathrm{P}$ & $\begin{array}{l}\text { Glyceroph } \\
\text { ospholipid } \\
\mathrm{s}\end{array}$ & $\begin{array}{l}\text { Glyceroph } \\
\text { osphates }\end{array}$ & $\begin{array}{l}7687 . \\
5\end{array}$ & $\begin{array}{l}15661 . \\
4\end{array}$ & 7428.6 & $\begin{array}{l}44705 . \\
5\end{array}$ & $\begin{array}{l}15566 . \\
0\end{array}$ & $\begin{array}{l}12187 . \\
2\end{array}$ & 0.0 & 0.0 & 0.0 & 0.0 & 0.0 & 0.0 \\
\hline PA(38:0) & 732.6 & 20.0 & C41H81O8P & $\begin{array}{l}\text { Glyceroph } \\
\text { ospholipid } \\
\text { s }\end{array}$ & $\begin{array}{l}\text { Glyceroph } \\
\text { osphates }\end{array}$ & $\begin{array}{l}7569 . \\
7\end{array}$ & 9523.9 & 0.0 & $\begin{array}{l}18871 . \\
9\end{array}$ & $\begin{array}{l}13741 . \\
9\end{array}$ & 0.0 & 0.0 & 0.0 & 0.0 & 0.0 & 0.0 & 0.0 \\
\hline $\operatorname{PA}(38: 1)$ & 730.6 & 19.4 & $\mathrm{C} 41 \mathrm{H} 79 \mathrm{O} 8 \mathrm{P}$ & $\begin{array}{l}\text { Glyceroph } \\
\text { ospholipid } \\
\text { s }\end{array}$ & $\begin{array}{l}\text { Glyceroph } \\
\text { osphates }\end{array}$ & $\begin{array}{l}10290 \\
.2\end{array}$ & $\begin{array}{l}10425 . \\
8\end{array}$ & 0.0 & $\begin{array}{l}31939 . \\
0\end{array}$ & $\begin{array}{l}14028 . \\
9\end{array}$ & $\begin{array}{l}13734 . \\
7\end{array}$ & 0.0 & 0.0 & 0.0 & 0.0 & 0.0 & 0.0 \\
\hline
\end{tabular}




\begin{tabular}{|c|c|c|c|c|c|c|c|c|c|c|c|c|c|c|c|c|c|}
\hline $\mathrm{PA}(38: 2)$ & 728.5 & 18.8 & $\mathrm{C} 41 \mathrm{H} 77 \mathrm{O} 8 \mathrm{P}$ & $\begin{array}{l}\text { Glyceroph } \\
\text { ospholipid } \\
\text { s }\end{array}$ & $\begin{array}{l}\text { Glyceroph } \\
\text { osphates }\end{array}$ & 0.0 & 7883.0 & 0.0 & $\begin{array}{l}29337 . \\
7\end{array}$ & $\begin{array}{l}11454 . \\
3\end{array}$ & $\begin{array}{l}12204 . \\
0\end{array}$ & 0.0 & 0.0 & 0.0 & 0.0 & 0.0 & 0.0 \\
\hline PA(38:4) & 724.5 & 13.1 & C41H73O8P & $\begin{array}{l}\text { Glyceroph } \\
\text { ospholipid } \\
\text { s }\end{array}$ & $\begin{array}{l}\text { Glyceroph } \\
\text { osphates }\end{array}$ & $\begin{array}{l}37940 \\
.0\end{array}$ & $\begin{array}{l}61924 . \\
9\end{array}$ & $\begin{array}{l}41223 . \\
5\end{array}$ & $\begin{array}{l}77747 . \\
1\end{array}$ & $\begin{array}{l}54683 . \\
3\end{array}$ & $\begin{array}{l}77025 . \\
3\end{array}$ & 0.0 & 0.0 & 0.0 & 0.0 & 0.0 & 0.0 \\
\hline PA(38:6) & 720.5 & 11.8 & С41H69O8P & $\begin{array}{l}\text { Glyceroph } \\
\text { ospholipid } \\
\text { s }\end{array}$ & $\begin{array}{l}\text { Glyceroph } \\
\text { osphates }\end{array}$ & $\begin{array}{l}19974 \\
.3\end{array}$ & $\begin{array}{l}20748 . \\
7\end{array}$ & $\begin{array}{l}15031 . \\
2\end{array}$ & $\begin{array}{l}15676 . \\
2\end{array}$ & $\begin{array}{l}16277 . \\
0\end{array}$ & $\begin{array}{l}32510 . \\
3\end{array}$ & 0.0 & 0.0 & 0.0 & 0.0 & 0.0 & 0.0 \\
\hline $\operatorname{PA}(39: 1)$ & 744.6 & 19.9 & $\mathrm{C} 42 \mathrm{H} 81 \mathrm{O} 8 \mathrm{P}$ & $\begin{array}{l}\text { Glyceroph } \\
\text { ospholipid } \\
\text { s }\end{array}$ & $\begin{array}{l}\text { Glyceroph } \\
\text { osphates }\end{array}$ & $\begin{array}{l}15359 \\
.0\end{array}$ & $\begin{array}{l}19298 . \\
7\end{array}$ & $\begin{array}{l}12269 . \\
3\end{array}$ & $\begin{array}{l}52598 . \\
6\end{array}$ & $\begin{array}{l}20926 . \\
6\end{array}$ & $\begin{array}{l}24618 . \\
2\end{array}$ & 0.0 & 0.0 & 0.0 & 0.0 & 0.0 & 0.0 \\
\hline PA(40:1) & 758.6 & 20.2 & C43H83O8P & $\begin{array}{l}\text { Glyceroph } \\
\text { ospholipid } \\
\text { s }\end{array}$ & $\begin{array}{l}\text { Glyceroph } \\
\text { osphates }\end{array}$ & $\begin{array}{l}10898 \\
.3\end{array}$ & $\begin{array}{l}17233 . \\
7\end{array}$ & 0.0 & $\begin{array}{l}32849 . \\
0\end{array}$ & $\begin{array}{l}21659 . \\
1\end{array}$ & $\begin{array}{l}18537 . \\
9\end{array}$ & 0.0 & 0.0 & 0.0 & 0.0 & 0.0 & 0.0 \\
\hline $\mathrm{PA}(40: 8)$ & 744.5 & 11.6 & $\mathrm{C} 43 \mathrm{H} 69 \mathrm{O} 8 \mathrm{P}$ & $\begin{array}{l}\text { Glyceroph } \\
\text { ospholipid } \\
\text { s }\end{array}$ & $\begin{array}{l}\text { Glyceroph } \\
\text { osphates }\end{array}$ & $\begin{array}{l}27035 \\
.5\end{array}$ & $\begin{array}{l}38783 . \\
5\end{array}$ & $\begin{array}{l}26442 . \\
3\end{array}$ & $\begin{array}{l}37757 . \\
8\end{array}$ & $\begin{array}{l}22143 . \\
0\end{array}$ & $\begin{array}{l}58883 . \\
1\end{array}$ & 0.0 & 0.0 & 0.0 & 0.0 & 0.0 & 0.0 \\
\hline $\mathrm{PA}(43: 0)$ & 802.6 & 21.1 & $\mathrm{C} 46 \mathrm{H} 9108 \mathrm{P}$ & $\begin{array}{l}\text { Glyceroph } \\
\text { ospholipid } \\
\text { s }\end{array}$ & $\begin{array}{l}\text { Glyceroph } \\
\text { osphates }\end{array}$ & $\begin{array}{l}26750 \\
.3\end{array}$ & $\begin{array}{l}35050 . \\
1\end{array}$ & 0.0 & $\begin{array}{l}34894 . \\
4\end{array}$ & $\begin{array}{l}26087 . \\
4\end{array}$ & $\begin{array}{l}19856 . \\
3\end{array}$ & 0.0 & 0.0 & 0.0 & 0.0 & 0.0 & 0.0 \\
\hline $\mathrm{PA}(\mathrm{O}-33: 1)$ & 646.5 & 15.7 & $\mathrm{C} 36 \mathrm{H} 71 \mathrm{O} 7 \mathrm{P}$ & $\begin{array}{l}\text { Glyceroph } \\
\text { ospholipid } \\
\text { s }\end{array}$ & $\begin{array}{l}\text { Glyceroph } \\
\text { osphates }\end{array}$ & $\begin{array}{l}27442 \\
.3\end{array}$ & $\begin{array}{l}39696 . \\
1\end{array}$ & $\begin{array}{l}34798 . \\
0\end{array}$ & $\begin{array}{l}41844 . \\
4\end{array}$ & $\begin{array}{l}26675 . \\
8\end{array}$ & $\begin{array}{l}35041 . \\
4\end{array}$ & 0.0 & 0.0 & 0.0 & 0.0 & 0.0 & 0.0 \\
\hline $\mathrm{PA}(\mathrm{O}-35: 0)$ & 676.5 & 13.9 & $\mathrm{C} 38 \mathrm{H} 77 \mathrm{O} 7 \mathrm{P}$ & $\begin{array}{l}\text { Glyceroph } \\
\text { ospholipid } \\
\text { s }\end{array}$ & $\begin{array}{l}\text { Glyceroph } \\
\text { osphates }\end{array}$ & $\begin{array}{l}40811 \\
.2\end{array}$ & $\begin{array}{l}34062 . \\
1\end{array}$ & $\begin{array}{l}56484 . \\
0\end{array}$ & $\begin{array}{l}52465 . \\
6\end{array}$ & $\begin{array}{l}37351 . \\
3\end{array}$ & $\begin{array}{l}48792 . \\
2\end{array}$ & 0.0 & 0.0 & 0.0 & 0.0 & 0.0 & 0.0 \\
\hline $\mathrm{PA}(\mathrm{O}-36: 3)$ & 684.5 & 16.2 & $\mathrm{C} 39 \mathrm{H} 73 \mathrm{O} 7 \mathrm{P}$ & $\begin{array}{l}\text { Glyceroph } \\
\text { ospholipid } \\
\text { s }\end{array}$ & $\begin{array}{l}\text { Glyceroph } \\
\text { osphates }\end{array}$ & $\begin{array}{l}73366 \\
.2\end{array}$ & $\begin{array}{l}132073 \\
.6\end{array}$ & $\begin{array}{l}163091 \\
.8\end{array}$ & $\begin{array}{l}207829 \\
.5\end{array}$ & $\begin{array}{l}46702 . \\
8\end{array}$ & $\begin{array}{l}95091 . \\
8\end{array}$ & 0.0 & 0.0 & 0.0 & 0.0 & 0.0 & 0.0 \\
\hline $\mathrm{PA}(\mathrm{O}-36: 4)$ & 682.5 & 15.9 & С39H71O7P & $\begin{array}{l}\text { Glyceroph } \\
\text { ospholipid } \\
\text { s }\end{array}$ & $\begin{array}{l}\text { Glyceroph } \\
\text { osphates }\end{array}$ & $\begin{array}{l}34585 \\
2.3\end{array}$ & $\begin{array}{l}605823 \\
.1\end{array}$ & $\begin{array}{l}741529 \\
.9\end{array}$ & $\begin{array}{l}786792 \\
.0\end{array}$ & $\begin{array}{l}142465 \\
.2\end{array}$ & $\begin{array}{l}628635 \\
.5\end{array}$ & 0.0 & 0.0 & 0.0 & 0.0 & 0.0 & 0.0 \\
\hline $\mathrm{PA}(\mathrm{O}-38: 6)$ & 706.5 & 14.9 & $\mathrm{C} 41 \mathrm{H} 71 \mathrm{O} 7 \mathrm{P}$ & $\begin{array}{l}\text { Glyceroph } \\
\text { ospholipid } \\
\text { S }\end{array}$ & $\begin{array}{l}\text { Glyceroph } \\
\text { osphates }\end{array}$ & $\begin{array}{l}90107 \\
.4\end{array}$ & $\begin{array}{l}162178 \\
.3\end{array}$ & $\begin{array}{l}181667 \\
.7\end{array}$ & $\begin{array}{l}192724 \\
.9\end{array}$ & $\begin{array}{l}49015 . \\
8\end{array}$ & $\begin{array}{l}147955 \\
.1\end{array}$ & 0.0 & 0.0 & 0.0 & 0.0 & 0.0 & 0.0 \\
\hline $\mathrm{PA}(\mathrm{P}-33: 2)$ & 642.5 & 14.1 & $\mathrm{C} 36 \mathrm{H} 67 \mathrm{O} 7 \mathrm{P}$ & $\begin{array}{l}\text { Glyceroph } \\
\text { ospholipid } \\
\text { s }\end{array}$ & $\begin{array}{l}\text { Glyceroph } \\
\text { osphates }\end{array}$ & $\begin{array}{l}85648 \\
.4\end{array}$ & $\begin{array}{l}113562 \\
.6\end{array}$ & $\begin{array}{l}82248 . \\
3\end{array}$ & $\begin{array}{l}98300 . \\
2\end{array}$ & $\begin{array}{l}47760 . \\
0\end{array}$ & $\begin{array}{l}83638 . \\
0\end{array}$ & 0.0 & 0.0 & 0.0 & 0.0 & 0.0 & 0.0 \\
\hline
\end{tabular}




\begin{tabular}{|c|c|c|c|c|c|c|c|c|c|c|c|c|c|c|c|c|c|}
\hline $\mathrm{PA}(\mathrm{P}-34: 2)$ & 656.5 & 14.7 & C37H69O7P & $\begin{array}{l}\text { Glyceroph } \\
\text { ospholipid } \\
\mathrm{s}\end{array}$ & $\begin{array}{l}\text { Ether lipid } \\
\text { metabolis } \\
\mathrm{m}\end{array}$ & $\begin{array}{l}66427 \\
.5\end{array}$ & $\begin{array}{l}90385 . \\
8\end{array}$ & $\begin{array}{l}84498 . \\
6\end{array}$ & $\begin{array}{l}119705 \\
.9\end{array}$ & $\begin{array}{l}54689 . \\
5\end{array}$ & $\begin{array}{l}74562 . \\
8\end{array}$ & 0.0 & 0.0 & 0.0 & 0.0 & 0.0 & 0.0 \\
\hline $\mathrm{PA}(\mathrm{P}-35: 2)$ & 670.5 & 15.4 & $\mathrm{C} 38 \mathrm{H} 71 \mathrm{O} 7 \mathrm{P}$ & $\begin{array}{l}\text { Glyceroph } \\
\text { ospholipid } \\
\mathrm{s}\end{array}$ & $\begin{array}{l}\text { Glyceroph } \\
\text { osphates }\end{array}$ & $\begin{array}{l}52676 \\
.4\end{array}$ & $\begin{array}{l}107174 \\
.7\end{array}$ & $\begin{array}{l}110507 \\
.1\end{array}$ & $\begin{array}{l}121448 \\
.1\end{array}$ & $\begin{array}{l}34766 . \\
2\end{array}$ & $\begin{array}{l}73407 . \\
2\end{array}$ & 0.0 & 0.0 & 0.0 & 0.0 & 0.0 & 0.0 \\
\hline $\mathrm{PA}(\mathrm{P}-36: 5)$ & 678.5 & 14.0 & С39H67O7P & $\begin{array}{l}\text { Glyceroph } \\
\text { ospholipid } \\
\mathrm{s}\end{array}$ & $\begin{array}{l}\text { Glyceroph } \\
\text { osphates }\end{array}$ & $\begin{array}{l}63455 \\
.4\end{array}$ & $\begin{array}{l}85255 . \\
6\end{array}$ & $\begin{array}{l}48111 . \\
2\end{array}$ & $\begin{array}{l}66496 . \\
1\end{array}$ & $\begin{array}{l}24848 . \\
2\end{array}$ & $\begin{array}{l}26346 . \\
9\end{array}$ & 0.0 & 0.0 & 0.0 & 0.0 & 0.0 & 0.0 \\
\hline $\mathrm{PA}(\mathrm{P}-38: 6)$ & 704.5 & 14.5 & C41H69O7P & $\begin{array}{l}\text { Glyceroph } \\
\text { ospholipid } \\
\mathrm{s}\end{array}$ & $\begin{array}{l}\text { Glyceroph } \\
\text { osphates }\end{array}$ & $\begin{array}{l}17117 \\
0.5\end{array}$ & $\begin{array}{l}242957 \\
.7\end{array}$ & $\begin{array}{l}217742 \\
.5\end{array}$ & $\begin{array}{l}256543 \\
.2\end{array}$ & $\begin{array}{l}47652 . \\
7\end{array}$ & $\begin{array}{l}183860 \\
.7\end{array}$ & 0.0 & 0.0 & 0.0 & 0.0 & 0.0 & 0.0 \\
\hline PC methyl(24:0) & 768.6 & 20.4 & C45H87NO6P & $\begin{array}{l}\text { Glyceroph } \\
\text { ospholipid } \\
\mathrm{s}\end{array}$ & $\begin{array}{l}\text { Glyceroph } \\
\text { osphocholi } \\
\text { nes }\end{array}$ & $\begin{array}{l}34409 \\
.9\end{array}$ & $\begin{array}{l}46952 . \\
2\end{array}$ & $\begin{array}{l}36453 . \\
3\end{array}$ & $\begin{array}{l}79939 . \\
2\end{array}$ & $\begin{array}{l}45674 . \\
1\end{array}$ & $\begin{array}{l}78053 . \\
8\end{array}$ & 0.0 & 0.0 & 0.0 & 0.0 & 0.0 & 0.0 \\
\hline $\mathrm{PC}(14: 1)$ & 451.3 & 5.9 & $\mathrm{C} 22 \mathrm{H} 46 \mathrm{NO} 6 \mathrm{P}$ & $\begin{array}{l}\text { Glyceroph } \\
\text { ospholipid } \\
\mathrm{s}\end{array}$ & $\begin{array}{l}\text { Glyceroph } \\
\text { osphocholi } \\
\text { nes }\end{array}$ & $\begin{array}{l}11121 \\
.0\end{array}$ & $\begin{array}{l}16409 . \\
3\end{array}$ & $\begin{array}{l}29564 . \\
9\end{array}$ & $\begin{array}{l}35914 . \\
3\end{array}$ & $\begin{array}{l}41450 . \\
8\end{array}$ & $\begin{array}{l}11766 . \\
9\end{array}$ & 0.0 & 0.0 & 0.0 & 0.0 & 0.0 & 0.0 \\
\hline $\mathrm{PC}(14: 2)$ & 453.3 & 5.7 & $\mathrm{C} 22 \mathrm{H} 48 \mathrm{NO} 6 \mathrm{P}$ & $\begin{array}{l}\text { Glyceroph } \\
\text { ospholipid } \\
\mathrm{s}\end{array}$ & $\begin{array}{l}\text { Glyceroph } \\
\text { osphocholi } \\
\text { nes }\end{array}$ & $\begin{array}{l}8708 . \\
6\end{array}$ & $\begin{array}{l}12659 . \\
6\end{array}$ & $\begin{array}{l}19851 . \\
9\end{array}$ & $\begin{array}{l}23760 . \\
5\end{array}$ & $\begin{array}{l}52433 . \\
4\end{array}$ & $\begin{array}{l}13234 . \\
8\end{array}$ & 0.0 & 0.0 & 0.0 & 0.0 & 0.0 & 0.0 \\
\hline $\mathrm{PC}(18: 2)$ & 519.3 & 4.2 & C26H50NO7P & $\begin{array}{l}\text { Glyceroph } \\
\text { ospholipid } \\
\text { s }\end{array}$ & $\begin{array}{l}\text { Glyceroph } \\
\text { osphocholi } \\
\text { nes }\end{array}$ & $\begin{array}{l}28398 \\
.6\end{array}$ & $\begin{array}{l}29165 . \\
1\end{array}$ & $\begin{array}{l}32967 . \\
9\end{array}$ & $\begin{array}{l}33073 . \\
1\end{array}$ & $\begin{array}{l}38136 . \\
3\end{array}$ & $\begin{array}{l}19998 . \\
9\end{array}$ & 0.0 & 0.0 & 0.0 & 0.0 & 0.0 & 0.0 \\
\hline $\begin{array}{l}\mathrm{PC}\left(18: 4\left(6 Z_{-} 9 \mathrm{Z}_{-} 12 \mathrm{Z} \_15 \mathrm{Z}\right) / \mathrm{P}-\right. \\
16: 0)\end{array}$ & 737.5 & 13.1 & $\mathrm{C} 42 \mathrm{H} 76 \mathrm{NO} 7 \mathrm{P}$ & $\begin{array}{l}\text { Glyceroph } \\
\text { ospholipid } \\
\text { s }\end{array}$ & $\begin{array}{l}\text { Glyceroph } \\
\text { osphocholi } \\
\text { nes }\end{array}$ & $\begin{array}{l}36398 \\
.2\end{array}$ & $\begin{array}{l}40833 \\
1\end{array}$ & $\begin{array}{l}59388 \\
6\end{array}$ & $\begin{array}{l}66346 . \\
7\end{array}$ & $\begin{array}{l}53829 . \\
1\end{array}$ & $\begin{array}{l}67005 \\
5\end{array}$ & 0.0 & 0.0 & 0.0 & 0.0 & 0.0 & 0.0 \\
\hline $\mathrm{PC}(19: 0)$ & 549.3 & 6.4 & $\mathrm{C} 27 \mathrm{H} 52 \mathrm{NO} 8 \mathrm{P}$ & $\begin{array}{l}\text { Glyceroph } \\
\text { ospholipid } \\
\mathrm{s}\end{array}$ & $\begin{array}{l}\text { Glyceroph } \\
\text { osphocholi } \\
\text { nes }\end{array}$ & 0.0 & 0.0 & 0.0 & 0.0 & 5458.5 & 0.0 & 0.0 & 0.0 & 0.0 & 0.0 & 0.0 & 0.0 \\
\hline $\mathrm{PC}(2: 0)$ & 321.1 & 1.4 & $\mathrm{C} 10 \mathrm{H} 22 \mathrm{NO} 7 \mathrm{P}$ & $\begin{array}{l}\text { Glyceroph } \\
\text { ospholipid } \\
\mathrm{s}\end{array}$ & $\begin{array}{l}\text { Glyceroph } \\
\text { osphocholi } \\
\text { nes }\end{array}$ & $\begin{array}{l}14198 \\
3.6\end{array}$ & $\begin{array}{l}53167 . \\
1\end{array}$ & 0.0 & 0.0 & 0.0 & 0.0 & 0.0 & 0.0 & 0.0 & 0.0 & 0.0 & 0.0 \\
\hline PC(21:0(CHO)) & 593.4 & 5.4 & C29H56NO9P & $\begin{array}{l}\text { Glyceroph } \\
\text { ospholipid } \\
\text { s }\end{array}$ & $\begin{array}{l}\text { Oxidized } \\
\text { glyceropho } \\
\text { spholipids }\end{array}$ & $\begin{array}{l}8055 . \\
5\end{array}$ & $\begin{array}{l}11362 . \\
6\end{array}$ & $\begin{array}{l}18338 . \\
3\end{array}$ & $\begin{array}{l}17655 . \\
5\end{array}$ & $\begin{array}{l}17952 . \\
6\end{array}$ & 8756.5 & 0.0 & 0.0 & 0.0 & 0.0 & 0.0 & 0.0 \\
\hline
\end{tabular}




\begin{tabular}{|c|c|c|c|c|c|c|c|c|c|c|c|c|c|c|c|c|c|}
\hline $\mathrm{PC}(21: 1)$ & 577.4 & 6.1 & $\mathrm{C} 29 \mathrm{H} 56 \mathrm{NO} 8 \mathrm{P}$ & $\begin{array}{l}\text { Glyceroph } \\
\text { ospholipid } \\
\text { s }\end{array}$ & $\begin{array}{l}\text { Glyceroph } \\
\text { osphocholi } \\
\text { nes }\end{array}$ & $\begin{array}{l}7019 \\
4\end{array}$ & 7167.0 & 0.0 & 0.0 & 0.0 & 0.0 & 0.0 & 0.0 & 0.0 & 0.0 & 0.0 & 0.0 \\
\hline $\mathrm{PC}(28: 0)$ & 677.5 & 11.4 & C36H72NO8P & $\begin{array}{l}\text { Glyceroph } \\
\text { ospholipid } \\
\text { s }\end{array}$ & $\begin{array}{l}\text { Glyceroph } \\
\text { osphocholi } \\
\text { nes }\end{array}$ & $\begin{array}{l}35793 \\
.9\end{array}$ & $\begin{array}{l}60614 . \\
7\end{array}$ & $\begin{array}{l}73492 . \\
3\end{array}$ & $\begin{array}{l}100396 \\
.9\end{array}$ & $\begin{array}{l}130944 \\
.5\end{array}$ & $\begin{array}{l}74041 . \\
4\end{array}$ & 9323.6 & 0.0 & $\begin{array}{l}10513 . \\
4\end{array}$ & $\begin{array}{l}10628 . \\
5\end{array}$ & $\begin{array}{l}22415 . \\
0\end{array}$ & $\begin{array}{l}14731 . \\
0\end{array}$ \\
\hline $\mathrm{PC}(30: 1)$ & 703.5 & 11.3 & $\mathrm{C} 38 \mathrm{H} 74 \mathrm{NO} 8 \mathrm{P}$ & $\begin{array}{l}\text { Glyceroph } \\
\text { ospholipid } \\
\text { s }\end{array}$ & $\begin{array}{l}\text { Glyceroph } \\
\text { osphocholi } \\
\text { nes }\end{array}$ & $\begin{array}{l}72200 \\
.0\end{array}$ & $\begin{array}{l}83218 \\
5\end{array}$ & $\begin{array}{l}141234 \\
.7\end{array}$ & $\begin{array}{l}158314 \\
.9\end{array}$ & $\begin{array}{l}223821 \\
.9\end{array}$ & $\begin{array}{l}150803 \\
.3\end{array}$ & 0.0 & 0.0 & 3803.4 & 0.0 & 0.0 & 0.0 \\
\hline $\mathrm{PC}(30: 2)$ & 691.6 & 13.1 & $\mathrm{C} 38 \mathrm{H} 78 \mathrm{NO} 7 \mathrm{P}$ & $\begin{array}{l}\text { Glyceroph } \\
\text { ospholipid } \\
\text { s }\end{array}$ & $\begin{array}{l}\text { Glyceroph } \\
\text { osphocholi } \\
\text { nes }\end{array}$ & $\begin{array}{l}20598 \\
25.1\end{array}$ & $\begin{array}{l}304360 \\
9.0\end{array}$ & $\begin{array}{l}286346 \\
1.8\end{array}$ & $\begin{array}{l}371678 \\
3.0\end{array}$ & $\begin{array}{l}490746 \\
8.0\end{array}$ & $\begin{array}{l}265137 \\
8.0\end{array}$ & $\begin{array}{l}15617 . \\
1\end{array}$ & 0.0 & 8434.8 & 0.0 & 5956.2 & 0.0 \\
\hline $\mathrm{PC}(32: 0)$ & 733.6 & 13.0 & $\mathrm{C} 40 \mathrm{H} 80 \mathrm{NO} 8 \mathrm{P}$ & $\begin{array}{l}\text { Glyceroph } \\
\text { ospholipid } \\
\text { s }\end{array}$ & $\begin{array}{l}\text { Glyceroph } \\
\text { osphocholi } \\
\text { nes }\end{array}$ & $\begin{array}{l}17519 \\
5.2\end{array}$ & $\begin{array}{l}250417 \\
.6\end{array}$ & $\begin{array}{l}260067 \\
.7\end{array}$ & $\begin{array}{l}252708 \\
.4\end{array}$ & $\begin{array}{l}195500 \\
.3\end{array}$ & $\begin{array}{l}207021 \\
.5\end{array}$ & 0.0 & 0.0 & 0.0 & 0.0 & 0.0 & 0.0 \\
\hline $\mathrm{PC}(32: 3)$ & 727.5 & 11.7 & $\mathrm{C} 40 \mathrm{H} 74 \mathrm{NO} 8 \mathrm{P}$ & $\begin{array}{l}\text { Glyceroph } \\
\text { ospholipid } \\
\text { s }\end{array}$ & $\begin{array}{l}\text { Glyceroph } \\
\text { osphocholi } \\
\text { nes }\end{array}$ & $\begin{array}{l}49114 \\
.5\end{array}$ & $\begin{array}{l}94561 . \\
5\end{array}$ & $\begin{array}{l}103416 \\
.1\end{array}$ & $\begin{array}{l}99762 . \\
8\end{array}$ & $\begin{array}{l}122408 \\
.4\end{array}$ & $\begin{array}{l}126748 \\
.2\end{array}$ & 0.0 & 0.0 & 0.0 & 0.0 & 0.0 & 0.0 \\
\hline $\mathrm{PC}(32: 4)$ & 715.6 & 13.6 & $\mathrm{C} 40 \mathrm{H} 78 \mathrm{NO} 7 \mathrm{P}$ & $\begin{array}{l}\text { Glyceroph } \\
\text { ospholipid } \\
\text { s }\end{array}$ & $\begin{array}{l}\text { Glyceroph } \\
\text { osphocholi } \\
\text { nes }\end{array}$ & $\begin{array}{l}16292 \\
71.4\end{array}$ & $\begin{array}{l}219235 \\
8.0\end{array}$ & $\begin{array}{l}329230 \\
5.0\end{array}$ & $\begin{array}{l}292490 \\
1.5\end{array}$ & $\begin{array}{l}130726 \\
4.4\end{array}$ & $\begin{array}{l}416395 \\
0.0\end{array}$ & $\begin{array}{l}16307 . \\
3\end{array}$ & 0.0 & 0.0 & 0.0 & 0.0 & 0.0 \\
\hline $\mathrm{PC}(34: 1)$ & 759.6 & 13.5 & $\mathrm{C} 42 \mathrm{H} 82 \mathrm{NO} 8 \mathrm{P}$ & $\begin{array}{l}\text { Glyceroph } \\
\text { ospholipid } \\
\text { s }\end{array}$ & $\begin{array}{l}\text { Glyceroph } \\
\text { osphocholi } \\
\text { nes }\end{array}$ & $\begin{array}{l}20048 \\
9.8\end{array}$ & $\begin{array}{l}283677 \\
.1\end{array}$ & $\begin{array}{l}315491 \\
.7\end{array}$ & $\begin{array}{l}291494 \\
.1\end{array}$ & $\begin{array}{l}224785 \\
.7\end{array}$ & $\begin{array}{l}330024 \\
.0\end{array}$ & $\begin{array}{l}14129 . \\
0\end{array}$ & 9994.7 & $\begin{array}{l}10130 . \\
2\end{array}$ & $\begin{array}{l}10177 . \\
5\end{array}$ & 9887.9 & 9827.8 \\
\hline $\mathrm{PC}(34: 2)$ & 757.6 & 13.2 & C42H80NO8P & $\begin{array}{l}\text { Glyceroph } \\
\text { ospholipid } \\
\text { s }\end{array}$ & $\begin{array}{l}\text { Glyceroph } \\
\text { osphocholi } \\
\text { nes }\end{array}$ & $\begin{array}{l}12141 \\
2.9\end{array}$ & $\begin{array}{l}140548 \\
.1\end{array}$ & $\begin{array}{l}167050 \\
.0\end{array}$ & $\begin{array}{l}174326 \\
.8\end{array}$ & $\begin{array}{l}154254 \\
.8\end{array}$ & $\begin{array}{l}179595 \\
.8\end{array}$ & $\begin{array}{l}34435 . \\
8\end{array}$ & $\begin{array}{l}35707 . \\
1\end{array}$ & $\begin{array}{l}42521 . \\
9\end{array}$ & $\begin{array}{l}41858 . \\
0\end{array}$ & $\begin{array}{l}37149 . \\
1\end{array}$ & $\begin{array}{l}37679 . \\
9\end{array}$ \\
\hline $\mathrm{PC}(34: 3)$ & 755.5 & 12.0 & $\mathrm{C} 42 \mathrm{H} 78 \mathrm{NO} 8 \mathrm{P}$ & $\begin{array}{l}\text { Glyceroph } \\
\text { ospholipid } \\
\text { s }\end{array}$ & $\begin{array}{l}\text { Glyceroph } \\
\text { osphocholi } \\
\text { nes }\end{array}$ & $\begin{array}{l}20962 \\
9.4\end{array}$ & $\begin{array}{l}318198 \\
.2\end{array}$ & $\begin{array}{l}478076 \\
.3\end{array}$ & $\begin{array}{l}590195 \\
.3\end{array}$ & $\begin{array}{l}724581 \\
.5\end{array}$ & $\begin{array}{l}607717 \\
.6\end{array}$ & 8222.1 & 0.0 & 0.0 & 0.0 & 0.0 & 0.0 \\
\hline $\mathrm{PC}(34: 4)$ & 753.5 & 12.4 & $\mathrm{C} 42 \mathrm{H} 76 \mathrm{NO} 8 \mathrm{P}$ & $\begin{array}{l}\text { Glyceroph } \\
\text { ospholipid } \\
\text { s }\end{array}$ & $\begin{array}{l}\text { Glyceroph } \\
\text { osphocholi } \\
\text { nes }\end{array}$ & $\begin{array}{l}12177 \\
5.6\end{array}$ & $\begin{array}{l}181060 \\
.4\end{array}$ & $\begin{array}{l}312900 \\
.3\end{array}$ & $\begin{array}{l}398224 \\
.9\end{array}$ & $\begin{array}{l}422347 \\
.8\end{array}$ & $\begin{array}{l}407910 \\
.8\end{array}$ & 0.0 & 0.0 & 0.0 & 0.0 & 0.0 & 0.0 \\
\hline $\mathrm{PC}(34: 5)$ & 751.5 & 12.0 & C42H74NO8P & $\begin{array}{l}\text { Glyceroph } \\
\text { ospholipid } \\
\text { s }\end{array}$ & $\begin{array}{l}\text { Glyceroph } \\
\text { osphocholi } \\
\text { nes }\end{array}$ & $\begin{array}{l}13658 \\
.5\end{array}$ & $\begin{array}{l}20540 . \\
1\end{array}$ & $\begin{array}{l}38136 . \\
9\end{array}$ & $\begin{array}{l}55295 . \\
2\end{array}$ & $\begin{array}{l}57512 . \\
2\end{array}$ & $\begin{array}{l}65203 . \\
3\end{array}$ & 0.0 & 0.0 & 0.0 & 0.0 & 0.0 & 0.0 \\
\hline $\mathrm{PC}(36: 1)$ & 787.6 & 14.9 & C44H86NO8P & $\begin{array}{l}\text { Glyceroph } \\
\text { ospholipid } \\
\text { s }\end{array}$ & $\begin{array}{l}\text { Glyceroph } \\
\text { osphocholi } \\
\text { nes }\end{array}$ & $\begin{array}{l}50779 \\
.5\end{array}$ & $\begin{array}{l}82177 . \\
8\end{array}$ & $\begin{array}{l}105474 \\
.7\end{array}$ & $\begin{array}{l}103758 \\
.5\end{array}$ & $\begin{array}{l}53341 . \\
4\end{array}$ & $\begin{array}{l}94055 . \\
6\end{array}$ & 0.0 & 0.0 & 0.0 & 0.0 & 0.0 & 0.0 \\
\hline
\end{tabular}




\begin{tabular}{|c|c|c|c|c|c|c|c|c|c|c|c|c|c|c|c|c|c|}
\hline $\operatorname{PC}(36: 4)$ & 781.6 & 12.8 & C44H80NO8P & $\begin{array}{l}\text { Glyceroph } \\
\text { ospholipid } \\
\mathrm{s}\end{array}$ & $\begin{array}{l}\text { Glyceroph } \\
\text { osphocholi } \\
\text { nes }\end{array}$ & $\begin{array}{l}16879 \\
35.4\end{array}$ & $\begin{array}{l}275875 \\
4.5\end{array}$ & $\begin{array}{l}431522 \\
4.0\end{array}$ & $\begin{array}{l}470705 \\
0.0\end{array}$ & $\begin{array}{l}357347 \\
4.0\end{array}$ & $\begin{array}{l}479924 \\
8.0\end{array}$ & $\begin{array}{l}15405 . \\
8\end{array}$ & 0.0 & 4468.7 & 0.0 & $\begin{array}{l}15826 . \\
3\end{array}$ & 0.0 \\
\hline $\mathrm{PC}(36: 6)$ & 777.5 & 11.5 & $\mathrm{C} 44 \mathrm{H} 76 \mathrm{NO} 8 \mathrm{P}$ & $\begin{array}{l}\text { Glyceroph } \\
\text { ospholipid } \\
\text { s }\end{array}$ & $\begin{array}{l}\text { Glyceroph } \\
\text { osphocholi } \\
\text { nes }\end{array}$ & $\begin{array}{l}24371 \\
.3\end{array}$ & $\begin{array}{l}18968 . \\
0\end{array}$ & $\begin{array}{l}37382 . \\
6\end{array}$ & $\begin{array}{l}37410 . \\
0\end{array}$ & $\begin{array}{l}27435 \\
3\end{array}$ & $\begin{array}{l}35072 . \\
0\end{array}$ & 0.0 & 0.0 & 0.0 & 0.0 & 0.0 & 0.0 \\
\hline $\mathrm{PC}(36: 7)$ & 775.5 & 10.7 & $\mathrm{C} 44 \mathrm{H} 74 \mathrm{NO} 8 \mathrm{P}$ & $\begin{array}{l}\text { Glyceroph } \\
\text { ospholipid } \\
\text { s }\end{array}$ & $\begin{array}{l}\text { Glyceroph } \\
\text { osphocholi } \\
\text { nes }\end{array}$ & 0.0 & 0.0 & 0.0 & 0.0 & 0.0 & 9088.0 & 0.0 & 0.0 & 0.0 & 0.0 & 0.0 & 0.0 \\
\hline $\mathrm{PC}(38: 2)$ & 813.6 & 15.3 & C46H88NO8P & $\begin{array}{l}\text { Glyceroph } \\
\text { ospholipid } \\
\mathrm{s}\end{array}$ & $\begin{array}{l}\text { Glyceroph } \\
\text { osphocholi } \\
\text { nes }\end{array}$ & $\begin{array}{l}27224 \\
9.0\end{array}$ & $\begin{array}{l}425433 \\
.1\end{array}$ & $\begin{array}{l}700617 \\
.2\end{array}$ & $\begin{array}{l}683974 \\
.4\end{array}$ & $\begin{array}{l}499962 \\
.0\end{array}$ & $\begin{array}{l}625879 \\
.7\end{array}$ & $\begin{array}{l}25936 \\
2\end{array}$ & $\begin{array}{l}26586 . \\
5\end{array}$ & $\begin{array}{l}25985 \\
4\end{array}$ & $\begin{array}{l}23474 . \\
5\end{array}$ & $\begin{array}{l}20064 . \\
0\end{array}$ & $\begin{array}{l}17546 . \\
8\end{array}$ \\
\hline $\mathrm{PC}(38: 6)$ & 805.6 & 12.5 & C46H80NO8P & $\begin{array}{l}\text { Glyceroph } \\
\text { ospholipid } \\
\text { s }\end{array}$ & $\begin{array}{l}\text { Glyceroph } \\
\text { osphocholi } \\
\text { nes }\end{array}$ & $\begin{array}{l}14266 \\
24.3\end{array}$ & $\begin{array}{l}196636 \\
9.5\end{array}$ & $\begin{array}{l}317354 \\
5.3\end{array}$ & $\begin{array}{l}321254 \\
6.5\end{array}$ & $\begin{array}{l}229058 \\
1.0\end{array}$ & $\begin{array}{l}334238 \\
5.0\end{array}$ & 9175.0 & 0.0 & 0.0 & 0.0 & 0.0 & 0.0 \\
\hline $\mathrm{PC}(38: 7)$ & 803.5 & 11.8 & C46H78NO8P & $\begin{array}{l}\text { Glyceroph } \\
\text { ospholipid } \\
\mathrm{s}\end{array}$ & $\begin{array}{l}\text { Glyceroph } \\
\text { osphocholi } \\
\text { nes }\end{array}$ & $\begin{array}{l}18919 \\
1.1\end{array}$ & $\begin{array}{l}207911 \\
.7\end{array}$ & $\begin{array}{l}296660 \\
.8\end{array}$ & $\begin{array}{l}333471 \\
.7\end{array}$ & $\begin{array}{l}310726 \\
.9\end{array}$ & $\begin{array}{l}354741 \\
.2\end{array}$ & 0.0 & 0.0 & 0.0 & 0.0 & 0.0 & 0.0 \\
\hline $\mathrm{PC}(40: 1)$ & 843.7 & 19.6 & C48H94NO8P & $\begin{array}{l}\text { Glyceroph } \\
\text { ospholipid } \\
\text { s }\end{array}$ & $\begin{array}{l}\text { Glyceroph } \\
\text { osphocholi } \\
\text { nes }\end{array}$ & $\begin{array}{l}15777 \\
.2\end{array}$ & 8618.2 & $\begin{array}{l}12167 . \\
9\end{array}$ & 8425.8 & 0.0 & $\begin{array}{l}10012 . \\
1\end{array}$ & 0.0 & 0.0 & 0.0 & 0.0 & 0.0 & 0.0 \\
\hline $\mathrm{PC}(40: 10)$ & 825.5 & 11.2 & $\mathrm{C} 48 \mathrm{H} 76 \mathrm{NO} 8 \mathrm{P}$ & $\begin{array}{l}\text { Glyceroph } \\
\text { ospholipid } \\
\text { s }\end{array}$ & $\begin{array}{l}\text { Glyceroph } \\
\text { osphocholi } \\
\text { nes }\end{array}$ & $\begin{array}{l}55939 \\
.6\end{array}$ & $\begin{array}{l}18567 . \\
9\end{array}$ & 9474.6 & 0.0 & 0.0 & 0.0 & 0.0 & 0.0 & 0.0 & 0.0 & 0.0 & 0.0 \\
\hline $\mathrm{PC}(40: 3)$ & 839.6 & 16.1 & C48H90NO8P & $\begin{array}{l}\text { Glyceroph } \\
\text { ospholipid } \\
\text { s }\end{array}$ & $\begin{array}{l}\text { Glyceroph } \\
\text { osphocholi } \\
\text { nes }\end{array}$ & $\begin{array}{l}93391 \\
.9\end{array}$ & $\begin{array}{l}133527 \\
.1\end{array}$ & $\begin{array}{l}214529 \\
.9\end{array}$ & $\begin{array}{l}231377 \\
.0\end{array}$ & $\begin{array}{l}99311 . \\
3\end{array}$ & $\begin{array}{l}243147 \\
.3\end{array}$ & 0.0 & 0.0 & 0.0 & 0.0 & 0.0 & 0.0 \\
\hline PC(40:4) & 837.6 & 15.0 & C48H88NO8P & $\begin{array}{l}\text { Glyceroph } \\
\text { ospholipid } \\
\text { s }\end{array}$ & $\begin{array}{l}\text { Glyceroph } \\
\text { osphocholi } \\
\text { nes }\end{array}$ & $\begin{array}{l}19314 \\
8.8\end{array}$ & $\begin{array}{l}295699 \\
.9\end{array}$ & $\begin{array}{l}486154 \\
.0\end{array}$ & $\begin{array}{l}527302 \\
.1\end{array}$ & $\begin{array}{l}341379 \\
.2\end{array}$ & $\begin{array}{l}517982 \\
.6\end{array}$ & 0.0 & 0.0 & 0.0 & 0.0 & 0.0 & 0.0 \\
\hline $\operatorname{PC}(40: 6)$ & 833.6 & 13.4 & C48H84NO8P & $\begin{array}{l}\text { Glyceroph } \\
\text { ospholipid } \\
\mathrm{s}\end{array}$ & $\begin{array}{l}\text { Glyceroph } \\
\text { osphocholi } \\
\text { nes }\end{array}$ & $\begin{array}{l}36007 \\
91.3\end{array}$ & $\begin{array}{l}552552 \\
2.0\end{array}$ & $\begin{array}{l}991972 \\
6.0\end{array}$ & $\begin{array}{l}105048 \\
62.0\end{array}$ & $\begin{array}{l}646297 \\
4.5\end{array}$ & $\begin{array}{l}112899 \\
38.0\end{array}$ & $\begin{array}{l}24483 . \\
7\end{array}$ & 0.0 & 0.0 & 0.0 & 0.0 & 0.0 \\
\hline PC(40:7) & 831.6 & 12.9 & C48H82NO8P & $\begin{array}{l}\text { Glyceroph } \\
\text { ospholipid } \\
\text { s }\end{array}$ & $\begin{array}{l}\text { Glyceroph } \\
\text { osphocholi } \\
\text { nes }\end{array}$ & $\begin{array}{l}55209 \\
55.5\end{array}$ & $\begin{array}{l}936155 \\
7.0\end{array}$ & $\begin{array}{l}159859 \\
52.0\end{array}$ & $\begin{array}{l}161803 \\
62.0\end{array}$ & $\begin{array}{l}945831 \\
1.0\end{array}$ & $\begin{array}{l}186600 \\
14.0\end{array}$ & $\begin{array}{l}37348 . \\
0\end{array}$ & 0.0 & 7810.8 & 0.0 & 0.0 & 0.0 \\
\hline $\mathrm{PC}(40: 8)$ & 829.6 & 12.3 & C48H80NO8P & $\begin{array}{l}\text { Glyceroph } \\
\text { ospholipid } \\
\text { s }\end{array}$ & $\begin{array}{l}\text { Glyceroph } \\
\text { osphocholi } \\
\text { nes }\end{array}$ & $\begin{array}{l}30834 \\
01.5\end{array}$ & $\begin{array}{l}496265 \\
8.5\end{array}$ & $\begin{array}{l}850196 \\
9.0\end{array}$ & $\begin{array}{l}881398 \\
9.0\end{array}$ & $\begin{array}{l}473502 \\
0.0\end{array}$ & $\begin{array}{l}959699 \\
1.0\end{array}$ & $\begin{array}{l}20477 . \\
6\end{array}$ & 0.0 & 0.0 & 0.0 & 0.0 & 0.0 \\
\hline
\end{tabular}




\begin{tabular}{|c|c|c|c|c|c|c|c|c|c|c|c|c|c|c|c|c|c|}
\hline $\mathrm{PC}(40: 9)$ & 827.5 & 11.7 & $\mathrm{C} 48 \mathrm{H} 78 \mathrm{NO} 8 \mathrm{P}$ & $\begin{array}{l}\text { Glyceroph } \\
\text { ospholipid } \\
\mathrm{s}\end{array}$ & $\begin{array}{l}\text { Glyceroph } \\
\text { osphocholi } \\
\text { nes }\end{array}$ & $\begin{array}{l}21043 \\
2.6\end{array}$ & $\begin{array}{l}150609 \\
.4\end{array}$ & $\begin{array}{l}215745 \\
.9\end{array}$ & $\begin{array}{l}193098 \\
.2\end{array}$ & $\begin{array}{l}89903 . \\
9\end{array}$ & $\begin{array}{l}231883 \\
.9\end{array}$ & 0.0 & 0.0 & 0.0 & 0.0 & 0.0 & 0.0 \\
\hline $\mathrm{PC}(42: 1)$ & 871.7 & 20.8 & C50H98NO8P & $\begin{array}{l}\text { Glyceroph } \\
\text { ospholipid } \\
\mathrm{s}\end{array}$ & $\begin{array}{l}\text { Glyceroph } \\
\text { osphocholi } \\
\text { nes }\end{array}$ & $\begin{array}{l}7616 . \\
1\end{array}$ & 0.0 & 5112.7 & 0.0 & 0.0 & 0.0 & 0.0 & 0.0 & 0.0 & 0.0 & 0.0 & 0.0 \\
\hline $\mathrm{PC}(42: 10)$ & 853.6 & 12.7 & C50H80NO8P & $\begin{array}{l}\text { Glyceroph } \\
\text { ospholipid } \\
\mathrm{s}\end{array}$ & $\begin{array}{l}\text { Glyceroph } \\
\text { osphocholi } \\
\text { nes }\end{array}$ & $\begin{array}{l}42650 \\
2.8\end{array}$ & $\begin{array}{l}654421 \\
.3\end{array}$ & $\begin{array}{l}114839 \\
4.3\end{array}$ & $\begin{array}{l}107650 \\
0.3\end{array}$ & $\begin{array}{l}755218 \\
.6\end{array}$ & $\begin{array}{l}130483 \\
6.5\end{array}$ & 0.0 & 0.0 & 0.0 & 0.0 & 0.0 & 0.0 \\
\hline $\mathrm{PC}(42: 2)$ & 869.7 & 20.2 & C50H96NO8P & $\begin{array}{l}\text { Glyceroph } \\
\text { ospholipid } \\
\mathrm{s}\end{array}$ & $\begin{array}{l}\text { Glyceroph } \\
\text { osphocholi } \\
\text { nes }\end{array}$ & $\begin{array}{l}7815 . \\
0\end{array}$ & 0.0 & 0.0 & 0.0 & 0.0 & 0.0 & 0.0 & 0.0 & 0.0 & 0.0 & 0.0 & 0.0 \\
\hline $\mathrm{PC}(42: 5)$ & 863.6 & 16.6 & C50H90NO8P & $\begin{array}{l}\text { Glyceroph } \\
\text { ospholipid } \\
\mathrm{s}\end{array}$ & $\begin{array}{l}\text { Glyceroph } \\
\text { osphocholi } \\
\text { nes }\end{array}$ & $\begin{array}{l}12346 \\
.6\end{array}$ & 8572.5 & 4210.3 & 7222.9 & 0.0 & 8343.2 & 0.0 & 0.0 & 0.0 & 0.0 & 0.0 & 0.0 \\
\hline $\mathrm{PC}(42: 7)$ & 859.6 & 14.0 & $\mathrm{C} 50 \mathrm{H} 86 \mathrm{NO} 8 \mathrm{P}$ & $\begin{array}{l}\text { Glyceroph } \\
\text { ospholipid } \\
\text { s }\end{array}$ & $\begin{array}{l}\text { Glyceroph } \\
\text { osphocholi } \\
\text { nes }\end{array}$ & $\begin{array}{l}78120 \\
.3\end{array}$ & $\begin{array}{l}137794 \\
.0\end{array}$ & $\begin{array}{l}241551 \\
.2\end{array}$ & $\begin{array}{l}244013 \\
.5\end{array}$ & $\begin{array}{l}112387 \\
.5\end{array}$ & $\begin{array}{l}285852 \\
.2\end{array}$ & 0.0 & 0.0 & 0.0 & 0.0 & 0.0 & 0.0 \\
\hline PC(44:5) & 891.7 & 18.5 & C52H94NO8P & $\begin{array}{l}\text { Glyceroph } \\
\text { ospholipid } \\
\mathrm{s}\end{array}$ & $\begin{array}{l}\text { Glyceroph } \\
\text { osphocholi } \\
\text { nes }\end{array}$ & $\begin{array}{l}63121 \\
.3\end{array}$ & $\begin{array}{l}18599 . \\
2\end{array}$ & $\begin{array}{l}13547 \\
8\end{array}$ & 0.0 & 0.0 & 0.0 & 0.0 & 0.0 & 0.0 & 0.0 & 0.0 & 0.0 \\
\hline $\mathrm{PC}(46: 5)$ & 919.7 & 20.0 & C54H98NO8P & $\begin{array}{l}\text { Glyceroph } \\
\text { ospholipid } \\
\mathrm{s}\end{array}$ & $\begin{array}{l}\text { Glyceroph } \\
\text { osphocholi } \\
\text { nes }\end{array}$ & $\begin{array}{l}17242 \\
3.9\end{array}$ & $\begin{array}{l}50943 . \\
9\end{array}$ & $\begin{array}{l}32441 . \\
9\end{array}$ & 0.0 & 0.0 & 0.0 & 0.0 & 0.0 & 0.0 & 0.0 & 0.0 & 0.0 \\
\hline $\begin{array}{l}\text { PC(o- } \\
\text { 22:0/20:4(8Z_11Z_14Z_17Z) } \\
)\end{array}$ & 851.7 & 18.2 & $\mathrm{C} 50 \mathrm{H} 94 \mathrm{NO} 7 \mathrm{P}$ & $\begin{array}{l}\text { Glyceroph } \\
\text { ospholipid } \\
\mathrm{s}\end{array}$ & $\begin{array}{l}\text { Glyceroph } \\
\text { osphocholi } \\
\text { nes }\end{array}$ & $\begin{array}{l}3711 . \\
5\end{array}$ & 0.0 & 0.0 & 0.0 & 0.0 & 0.0 & 0.0 & 0.0 & 0.0 & 0.0 & 0.0 & 0.0 \\
\hline $\mathrm{PC}(\mathrm{O}-29: 0)$ & 677.5 & 12.9 & $\mathrm{C} 37 \mathrm{H} 76 \mathrm{NO} 7 \mathrm{P}$ & $\begin{array}{l}\text { Glyceroph } \\
\text { ospholipid } \\
\mathrm{s}\end{array}$ & $\begin{array}{l}\text { Glyceroph } \\
\text { osphocholi } \\
\text { nes }\end{array}$ & $\begin{array}{l}70028 \\
.6\end{array}$ & $\begin{array}{l}135692 \\
.2\end{array}$ & $\begin{array}{l}149745 \\
.6\end{array}$ & $\begin{array}{l}273360 \\
.8\end{array}$ & $\begin{array}{l}218551 \\
.0\end{array}$ & $\begin{array}{l}196385 \\
.6\end{array}$ & 0.0 & 0.0 & 0.0 & 0.0 & 0.0 & 0.0 \\
\hline $\mathrm{PC}(\mathrm{O}-31: 0)$ & 705.6 & 13.9 & $\mathrm{C} 39 \mathrm{H} 80 \mathrm{NO} 7 \mathrm{P}$ & $\begin{array}{l}\text { Glyceroph } \\
\text { ospholipid } \\
\text { s }\end{array}$ & $\begin{array}{l}\text { Glyceroph } \\
\text { osphocholi } \\
\text { nes }\end{array}$ & $\begin{array}{l}29924 \\
8.8\end{array}$ & $\begin{array}{l}642548 \\
.0\end{array}$ & $\begin{array}{l}744752 \\
.7\end{array}$ & $\begin{array}{l}904533 \\
.2\end{array}$ & $\begin{array}{l}745567 \\
.7\end{array}$ & $\begin{array}{l}687700 \\
.8\end{array}$ & 0.0 & 0.0 & 0.0 & 0.0 & 0.0 & 0.0 \\
\hline $\mathrm{PC}(\mathrm{O}-32: 0)$ & 719.6 & 14.3 & $\mathrm{C} 40 \mathrm{H} 82 \mathrm{NO} 7 \mathrm{P}$ & $\begin{array}{l}\text { Glyceroph } \\
\text { ospholipid } \\
\text { s }\end{array}$ & $\begin{array}{l}\text { Glyceroph } \\
\text { osphocholi } \\
\text { nes }\end{array}$ & $\begin{array}{l}38133 \\
1.8\end{array}$ & $\begin{array}{l}748534 \\
.1\end{array}$ & $\begin{array}{l}103945 \\
1.6\end{array}$ & $\begin{array}{l}117648 \\
4.5\end{array}$ & $\begin{array}{l}845538 \\
.4\end{array}$ & $\begin{array}{l}778214 \\
.7\end{array}$ & 4279.6 & 0.0 & 0.0 & 0.0 & 0.0 & 0.0 \\
\hline $\mathrm{PC}(\mathrm{O}-38: 2)$ & 799.6 & 16.4 & $\mathrm{C} 46 \mathrm{H} 90 \mathrm{NO} 7 \mathrm{P}$ & $\begin{array}{l}\text { Glyceroph } \\
\text { ospholipid } \\
\text { s }\end{array}$ & $\begin{array}{l}\text { Glyceroph } \\
\text { osphocholi } \\
\text { nes }\end{array}$ & $\begin{array}{l}17921 \\
5.7\end{array}$ & $\begin{array}{l}237541 \\
.7\end{array}$ & $\begin{array}{l}349304 \\
.9\end{array}$ & $\begin{array}{l}343003 \\
.4\end{array}$ & $\begin{array}{l}206568 \\
.9\end{array}$ & $\begin{array}{l}379893 \\
.2\end{array}$ & 0.0 & 0.0 & 0.0 & 0.0 & 0.0 & 0.0 \\
\hline
\end{tabular}




\begin{tabular}{|c|c|c|c|c|c|c|c|c|c|c|c|c|c|c|c|c|c|}
\hline $\mathrm{PC}(\mathrm{O}-42: 3)$ & 870.7 & 16.7 & $\mathrm{C} 50 \mathrm{H} 96 \mathrm{NO} 7 \mathrm{P}$ & $\begin{array}{l}\text { Glyceroph } \\
\text { ospholipid } \\
\mathrm{s}\end{array}$ & $\begin{array}{l}\text { Glyceroph } \\
\text { osphocholi } \\
\text { nes }\end{array}$ & $\begin{array}{l}59542 \\
.2\end{array}$ & $\begin{array}{l}93552 . \\
9\end{array}$ & $\begin{array}{l}135417 \\
.7\end{array}$ & $\begin{array}{l}142694 \\
.8\end{array}$ & $\begin{array}{l}34140 . \\
3\end{array}$ & $\begin{array}{l}168605 \\
.0\end{array}$ & 0.0 & 0.0 & 0.0 & 0.0 & 0.0 & 0.0 \\
\hline $\mathrm{PC}(\mathrm{P}-30: 0)$ & 689.5 & 12.9 & $\mathrm{C} 38 \mathrm{H} 76 \mathrm{NO} 7 \mathrm{P}$ & $\begin{array}{l}\text { Glyceroph } \\
\text { ospholipid } \\
\text { s }\end{array}$ & $\begin{array}{l}\text { Glyceroph } \\
\text { osphocholi } \\
\text { nes }\end{array}$ & $\begin{array}{l}27462 \\
18.0\end{array}$ & $\begin{array}{l}390703 \\
8.5\end{array}$ & $\begin{array}{l}447930 \\
2.5\end{array}$ & $\begin{array}{l}557433 \\
6.0\end{array}$ & $\begin{array}{l}783919 \\
0.0\end{array}$ & $\begin{array}{l}475882 \\
4.5\end{array}$ & $\begin{array}{l}17526 . \\
1\end{array}$ & 0.0 & 6419.3 & 0.0 & 5484.6 & 0.0 \\
\hline $\mathrm{PC}(\mathrm{P}-32: 0)$ & 717.6 & 13.8 & $\mathrm{C} 40 \mathrm{H} 80 \mathrm{NO} 7 \mathrm{P}$ & $\begin{array}{l}\text { Glyceroph } \\
\text { ospholipid } \\
\text { s }\end{array}$ & $\begin{array}{l}\text { Glyceroph } \\
\text { osphocholi } \\
\text { nes }\end{array}$ & $\begin{array}{l}22033 \\
49.0\end{array}$ & $\begin{array}{l}303138 \\
8.5\end{array}$ & $\begin{array}{l}356620 \\
4.5\end{array}$ & $\begin{array}{l}315870 \\
0.8\end{array}$ & $\begin{array}{l}168806 \\
7.8\end{array}$ & $\begin{array}{l}410265 \\
1.8\end{array}$ & $\begin{array}{l}27111 . \\
9\end{array}$ & 0.0 & 3379.5 & 0.0 & 7445.3 & 0.0 \\
\hline $\mathrm{PC}(\mathrm{P}-32: 2)$ & 713.5 & 13.1 & $\mathrm{C} 40 \mathrm{H} 76 \mathrm{NO} 7 \mathrm{P}$ & $\begin{array}{l}\text { Glyceroph } \\
\text { ospholipid } \\
\text { s }\end{array}$ & $\begin{array}{l}\text { Glyceroph } \\
\text { osphocholi } \\
\text { nes }\end{array}$ & $\begin{array}{l}12303 \\
2.7\end{array}$ & $\begin{array}{l}182742 \\
.9\end{array}$ & $\begin{array}{l}266143 \\
.3\end{array}$ & $\begin{array}{l}284712 \\
.2\end{array}$ & $\begin{array}{l}216613 \\
.5\end{array}$ & $\begin{array}{l}313706 \\
.4\end{array}$ & 0.0 & 0.0 & 0.0 & 0.0 & 0.0 & 0.0 \\
\hline $\mathrm{PC}(\mathrm{P}-34: 0)$ & 745.6 & 15.0 & $\mathrm{C} 42 \mathrm{H} 84 \mathrm{NO} 7 \mathrm{P}$ & $\begin{array}{l}\text { Glyceroph } \\
\text { ospholipid } \\
\text { s }\end{array}$ & $\begin{array}{l}\text { Glyceroph } \\
\text { osphocholi } \\
\text { nes }\end{array}$ & $\begin{array}{l}24945 \\
0.8\end{array}$ & $\begin{array}{l}393499 \\
.0\end{array}$ & $\begin{array}{l}500515 \\
.6\end{array}$ & $\begin{array}{l}575296 \\
.8\end{array}$ & $\begin{array}{l}459366 \\
.1\end{array}$ & $\begin{array}{l}515910 \\
.5\end{array}$ & 0.0 & 0.0 & 0.0 & 0.0 & 0.0 & 0.0 \\
\hline $\mathrm{PC}(\mathrm{P}-34: 1)$ & 743.6 & 14.6 & $\mathrm{C} 42 \mathrm{H} 82 \mathrm{NO} 7 \mathrm{P}$ & $\begin{array}{l}\text { Glyceroph } \\
\text { ospholipid } \\
\text { s }\end{array}$ & $\begin{array}{l}\text { Glyceroph } \\
\text { osphocholi } \\
\text { nes }\end{array}$ & $\begin{array}{l}30124 \\
3.2\end{array}$ & $\begin{array}{l}554287 \\
.7\end{array}$ & $\begin{array}{l}929877 \\
.9\end{array}$ & $\begin{array}{l}108223 \\
8.6\end{array}$ & $\begin{array}{l}720351 \\
.4\end{array}$ & $\begin{array}{l}981585 \\
.8\end{array}$ & 0.0 & 0.0 & 0.0 & 0.0 & 0.0 & 0.0 \\
\hline $\mathrm{PC}(\mathrm{P}-34: 2)$ & 741.6 & 14.1 & $\mathrm{C} 42 \mathrm{H} 80 \mathrm{NO} 7 \mathrm{P}$ & $\begin{array}{l}\text { Glyceroph } \\
\text { ospholipid } \\
\text { s }\end{array}$ & $\begin{array}{l}\text { Glyceroph } \\
\text { osphocholi } \\
\text { nes }\end{array}$ & $\begin{array}{l}91216 \\
.7\end{array}$ & $\begin{array}{l}159600 \\
.2\end{array}$ & $\begin{array}{l}251841 \\
.9\end{array}$ & $\begin{array}{l}265917 \\
.7\end{array}$ & $\begin{array}{l}185448 \\
.2\end{array}$ & $\begin{array}{l}299390 \\
.2\end{array}$ & 0.0 & 0.0 & 0.0 & 0.0 & 0.0 & 0.0 \\
\hline PC(P-36:2) & 769.6 & 15.0 & $\mathrm{C} 44 \mathrm{H} 84 \mathrm{NO} 7 \mathrm{P}$ & $\begin{array}{l}\text { Glyceroph } \\
\text { ospholipid } \\
\text { s }\end{array}$ & $\begin{array}{l}\text { Glyceroph } \\
\text { osphocholi } \\
\text { nes }\end{array}$ & $\begin{array}{l}34022 \\
.7\end{array}$ & $\begin{array}{l}47067 . \\
7\end{array}$ & $\begin{array}{l}62084 . \\
4\end{array}$ & $\begin{array}{l}60685 . \\
0\end{array}$ & $\begin{array}{l}36648 . \\
7\end{array}$ & $\begin{array}{l}71846 . \\
5\end{array}$ & 0.0 & 0.0 & 0.0 & 0.0 & 0.0 & 0.0 \\
\hline $\mathrm{PC}(\mathrm{P}-36: 3)$ & 767.6 & 14.4 & $\mathrm{C} 44 \mathrm{H} 82 \mathrm{NO} 7 \mathrm{P}$ & $\begin{array}{l}\text { Glyceroph } \\
\text { ospholipid } \\
\text { s }\end{array}$ & $\begin{array}{l}\text { Glyceroph } \\
\text { osphocholi } \\
\text { nes }\end{array}$ & $\begin{array}{l}15156 \\
1.0\end{array}$ & $\begin{array}{l}280332 \\
.4\end{array}$ & $\begin{array}{l}439216 \\
.4\end{array}$ & $\begin{array}{l}532309 \\
.6\end{array}$ & $\begin{array}{l}281255 \\
.3\end{array}$ & $\begin{array}{l}542815 \\
.3\end{array}$ & 0.0 & 0.0 & 0.0 & 0.0 & 0.0 & 0.0 \\
\hline $\mathrm{PC}(\mathrm{P}-36: 5)$ & 763.6 & 13.6 & $\mathrm{C} 44 \mathrm{H} 78 \mathrm{NO} 7 \mathrm{P}$ & $\begin{array}{l}\text { Glyceroph } \\
\text { ospholipid } \\
\text { s }\end{array}$ & $\begin{array}{l}\text { Glyceroph } \\
\text { osphocholi } \\
\text { nes }\end{array}$ & $\begin{array}{l}28320 \\
.0\end{array}$ & $\begin{array}{l}39082 . \\
1\end{array}$ & $\begin{array}{l}20880 . \\
4\end{array}$ & 0.0 & 0.0 & $\begin{array}{l}29614 . \\
5\end{array}$ & 0.0 & 0.0 & 0.0 & 0.0 & 0.0 & 0.0 \\
\hline $\mathrm{PC}(\mathrm{P}-38: 2)$ & 797.6 & 15.8 & $\mathrm{C} 46 \mathrm{H} 88 \mathrm{NO} 7 \mathrm{P}$ & $\begin{array}{l}\text { Glyceroph } \\
\text { ospholipid } \\
\text { s }\end{array}$ & $\begin{array}{l}\text { Glyceroph } \\
\text { osphocholi } \\
\text { nes }\end{array}$ & $\begin{array}{l}40707 \\
6.5\end{array}$ & $\begin{array}{l}604010 \\
.9\end{array}$ & $\begin{array}{l}886525 \\
.1\end{array}$ & $\begin{array}{l}996629 \\
.4\end{array}$ & $\begin{array}{l}548730 \\
.5\end{array}$ & $\begin{array}{l}896656 \\
.6\end{array}$ & 2942.8 & 0.0 & 0.0 & 0.0 & 0.0 & 0.0 \\
\hline PC(P-38:3) & 795.6 & 15.3 & $\mathrm{C} 46 \mathrm{H} 86 \mathrm{NO} 7 \mathrm{P}$ & $\begin{array}{l}\text { Glyceroph } \\
\text { ospholipid } \\
\text { s }\end{array}$ & $\begin{array}{l}\text { Glyceroph } \\
\text { osphocholi } \\
\text { nes }\end{array}$ & $\begin{array}{l}67930 \\
0.8\end{array}$ & $\begin{array}{l}105959 \\
0.5\end{array}$ & $\begin{array}{l}144319 \\
3.1\end{array}$ & $\begin{array}{l}151436 \\
9.6\end{array}$ & $\begin{array}{l}945773 \\
.3\end{array}$ & $\begin{array}{l}149626 \\
8.3\end{array}$ & 0.0 & 0.0 & 0.0 & 0.0 & 0.0 & 0.0 \\
\hline PC(P-38:4) & 810.6 & 12.9 & $\mathrm{C} 46 \mathrm{H} 84 \mathrm{NO} 7 \mathrm{P}$ & $\begin{array}{l}\text { Glyceroph } \\
\text { ospholipid } \\
\text { s }\end{array}$ & $\begin{array}{l}\text { Glyceroph } \\
\text { osphocholi } \\
\text { nes }\end{array}$ & $\begin{array}{l}24262 \\
0.8\end{array}$ & $\begin{array}{l}383909 \\
.6\end{array}$ & $\begin{array}{l}406831 \\
.2\end{array}$ & $\begin{array}{l}518446 \\
.3\end{array}$ & $\begin{array}{l}631744 \\
.1\end{array}$ & $\begin{array}{l}456164 \\
.1\end{array}$ & 0.0 & 0.0 & 0.0 & 0.0 & 0.0 & 0.0 \\
\hline
\end{tabular}




\begin{tabular}{|c|c|c|c|c|c|c|c|c|c|c|c|c|c|c|c|c|c|}
\hline $\mathrm{PC}(\mathrm{P}-40: 2)$ & 842.7 & 15.0 & C48H92NO7P & $\begin{array}{l}\text { Glyceroph } \\
\text { ospholipid } \\
\mathrm{s}\end{array}$ & $\begin{array}{l}\text { Glyceroph } \\
\text { osphocholi } \\
\text { nes }\end{array}$ & $\begin{array}{l}25085 \\
4.7\end{array}$ & $\begin{array}{l}452539 \\
.3\end{array}$ & $\begin{array}{l}736835 \\
.2\end{array}$ & $\begin{array}{l}752995 \\
.7\end{array}$ & $\begin{array}{l}272759 \\
.5\end{array}$ & $\begin{array}{l}987109 \\
.6\end{array}$ & 0.0 & 0.0 & 0.0 & 0.0 & 0.0 & 0.0 \\
\hline $\mathrm{PC}(\mathrm{P}-40: 3)$ & 823.6 & 16.6 & C48H90NO7P & $\begin{array}{l}\text { Glyceroph } \\
\text { ospholipid } \\
\mathrm{s}\end{array}$ & $\begin{array}{l}\text { Glyceroph } \\
\text { osphocholi } \\
\text { nes }\end{array}$ & $\begin{array}{l}21027 \\
.4\end{array}$ & $\begin{array}{l}25905 . \\
2\end{array}$ & $\begin{array}{l}36673 . \\
5\end{array}$ & $\begin{array}{l}40194 . \\
0\end{array}$ & $\begin{array}{l}18592 . \\
8\end{array}$ & $\begin{array}{l}34176 . \\
1\end{array}$ & 0.0 & 0.0 & 0.0 & 0.0 & 0.0 & 0.0 \\
\hline PC(P-40:4) & 821.6 & 15.6 & C48H88NO7P & $\begin{array}{l}\text { Glyceroph } \\
\text { ospholipid } \\
\mathrm{s}\end{array}$ & $\begin{array}{l}\text { Glyceroph } \\
\text { osphocholi } \\
\text { nes }\end{array}$ & $\begin{array}{l}31656 \\
.5\end{array}$ & $\begin{array}{l}30137 \\
8\end{array}$ & $\begin{array}{l}34544 . \\
5\end{array}$ & $\begin{array}{l}31386 . \\
8\end{array}$ & $\begin{array}{l}19437 . \\
9\end{array}$ & $\begin{array}{l}23115 \\
8\end{array}$ & 0.0 & 0.0 & 0.0 & 0.0 & 0.0 & 0.0 \\
\hline $\mathrm{PC}(\mathrm{P}-40: 5)$ & 819.6 & 15.7 & C48H86NO7P & $\begin{array}{l}\text { Glyceroph } \\
\text { ospholipid } \\
\mathrm{s}\end{array}$ & $\begin{array}{l}\text { Glyceroph } \\
\text { osphocholi } \\
\text { nes }\end{array}$ & $\begin{array}{l}80605 \\
.0\end{array}$ & $\begin{array}{l}123382 \\
.3\end{array}$ & $\begin{array}{l}201110 \\
.0\end{array}$ & $\begin{array}{l}227648 \\
.7\end{array}$ & $\begin{array}{l}131835 \\
.9\end{array}$ & $\begin{array}{l}213110 \\
.3\end{array}$ & 0.0 & 0.0 & 0.0 & 0.0 & 0.0 & 0.0 \\
\hline $\mathrm{PC}(\mathrm{P}-40: 6)$ & 834.6 & 9.9 & C48H84NO7P & $\begin{array}{l}\text { Glyceroph } \\
\text { ospholipid } \\
\mathrm{s}\end{array}$ & $\begin{array}{l}\text { Glyceroph } \\
\text { osphocholi } \\
\text { nes }\end{array}$ & $\begin{array}{l}25913 \\
.2\end{array}$ & $\begin{array}{l}40987 . \\
0\end{array}$ & $\begin{array}{l}40859 \\
4\end{array}$ & $\begin{array}{l}52562 . \\
6\end{array}$ & $\begin{array}{l}23320 \\
3\end{array}$ & $\begin{array}{l}118526 \\
.2\end{array}$ & 0.0 & 0.0 & 0.0 & 0.0 & 0.0 & 0.0 \\
\hline $\mathrm{PC}(\mathrm{P}-42: 4)$ & 849.7 & 17.5 & C50H92NO7P & $\begin{array}{l}\text { Glyceroph } \\
\text { ospholipid } \\
\text { s }\end{array}$ & $\begin{array}{l}\text { Glyceroph } \\
\text { osphocholi } \\
\text { nes }\end{array}$ & $\begin{array}{l}11173 \\
.0\end{array}$ & 3403.7 & 0.0 & 0.0 & 0.0 & 0.0 & 0.0 & 0.0 & 0.0 & 0.0 & 0.0 & 0.0 \\
\hline $\operatorname{PE}(20: 0)$ & 523.3 & 6.3 & C25H50NO8P & $\begin{array}{l}\text { Glyceroph } \\
\text { ospholipid } \\
\mathrm{s}\end{array}$ & $\begin{array}{l}\text { Glyceroph } \\
\text { osphoetha } \\
\text { nolamines }\end{array}$ & $\begin{array}{l}11395 \\
7.8\end{array}$ & $\begin{array}{l}98512 . \\
3\end{array}$ & $\begin{array}{l}239758 \\
.6\end{array}$ & $\begin{array}{l}285535 \\
.7\end{array}$ & $\begin{array}{l}206117 \\
.9\end{array}$ & $\begin{array}{l}82379 . \\
8\end{array}$ & 0.0 & 0.0 & 0.0 & 0.0 & 0.0 & 0.0 \\
\hline $\operatorname{PE}(26: 0)$ & 607.4 & 8.1 & C31H62NO8P & $\begin{array}{l}\text { Glyceroph } \\
\text { ospholipid } \\
\mathrm{s}\end{array}$ & $\begin{array}{l}\text { Glyceroph } \\
\text { osphoetha } \\
\text { nolamines }\end{array}$ & 0.0 & 6145.6 & 0.0 & 0.0 & 0.0 & 0.0 & 0.0 & 0.0 & 0.0 & 0.0 & 0.0 & 0.0 \\
\hline $\operatorname{PE}(26: 1)$ & 605.4 & 7.2 & C31H60NO8P & $\begin{array}{l}\text { Glyceroph } \\
\text { ospholipid } \\
\mathrm{s}\end{array}$ & $\begin{array}{l}\text { Glyceroph } \\
\text { osphoetha } \\
\text { nolamines }\end{array}$ & $\begin{array}{l}26018 \\
.5\end{array}$ & $\begin{array}{l}13513 . \\
1\end{array}$ & 8348.0 & 6230.4 & 7246.4 & 3029.4 & 0.0 & 0.0 & 0.0 & 0.0 & 0.0 & 0.0 \\
\hline $\operatorname{PE}(30: 0)$ & 663.5 & 10.8 & C35H70NO8P & $\begin{array}{l}\text { Glyceroph } \\
\text { ospholipid } \\
\mathrm{s}\end{array}$ & $\begin{array}{l}\text { Glyceroph } \\
\text { osphoetha } \\
\text { nolamines }\end{array}$ & $\begin{array}{l}57700 \\
.7\end{array}$ & $\begin{array}{l}96581 . \\
0\end{array}$ & $\begin{array}{l}99335 . \\
7\end{array}$ & $\begin{array}{l}125167 \\
.4\end{array}$ & $\begin{array}{l}249488 \\
.3\end{array}$ & $\begin{array}{l}98964 . \\
7\end{array}$ & $\begin{array}{l}17790 . \\
0\end{array}$ & 0.0 & $\begin{array}{l}10928 . \\
7\end{array}$ & $\begin{array}{l}13433 . \\
1\end{array}$ & $\begin{array}{l}27613 . \\
8\end{array}$ & $\begin{array}{l}16738 . \\
9\end{array}$ \\
\hline $\operatorname{PE}(32: 0)$ & 691.5 & 12.0 & C37H74NO8P & $\begin{array}{l}\text { Glyceroph } \\
\text { ospholipid } \\
\text { s }\end{array}$ & $\begin{array}{l}\text { Glyceroph } \\
\text { osphoetha } \\
\text { nolamines }\end{array}$ & $\begin{array}{l}28558 \\
8.3\end{array}$ & $\begin{array}{l}463930 \\
.0\end{array}$ & $\begin{array}{l}498455 \\
.0\end{array}$ & $\begin{array}{l}524913 \\
.8\end{array}$ & $\begin{array}{l}734141 \\
.8\end{array}$ & $\begin{array}{l}470388 \\
.9\end{array}$ & $\begin{array}{l}13323 . \\
2\end{array}$ & 6943.0 & $\begin{array}{l}10026 . \\
0\end{array}$ & 9758.6 & $\begin{array}{l}15479 . \\
3\end{array}$ & 9370.8 \\
\hline $\operatorname{PE}(32: 1)$ & 689.5 & 11.4 & C37H72NO8P & $\begin{array}{l}\text { Glyceroph } \\
\text { ospholipid } \\
\mathrm{s}\end{array}$ & $\begin{array}{l}\text { Glyceroph } \\
\text { osphoetha } \\
\text { nolamines }\end{array}$ & $\begin{array}{l}68234 \\
.9\end{array}$ & $\begin{array}{l}106401 \\
.9\end{array}$ & $\begin{array}{l}176045 \\
.1\end{array}$ & $\begin{array}{l}180931 \\
.3\end{array}$ & $\begin{array}{l}162083 \\
.2\end{array}$ & $\begin{array}{l}163983 \\
.4\end{array}$ & 0.0 & 0.0 & 0.0 & 0.0 & 0.0 & 0.0 \\
\hline $\operatorname{PE}(34: 0)$ & 719.5 & 13.1 & C39H78NO8P & $\begin{array}{l}\text { Glyceroph } \\
\text { ospholipid } \\
\mathrm{s}\end{array}$ & $\begin{array}{l}\text { Glyceroph } \\
\text { osphoetha } \\
\text { nolamines }\end{array}$ & $\begin{array}{l}20255 \\
8.5\end{array}$ & $\begin{array}{l}283305 \\
.3\end{array}$ & $\begin{array}{l}314649 \\
.5\end{array}$ & $\begin{array}{l}320757 \\
.1\end{array}$ & $\begin{array}{l}258816 \\
.9\end{array}$ & $\begin{array}{l}269456 \\
.7\end{array}$ & 0.0 & 0.0 & 0.0 & 0.0 & 0.0 & 0.0 \\
\hline
\end{tabular}




\begin{tabular}{|c|c|c|c|c|c|c|c|c|c|c|c|c|c|c|c|c|c|}
\hline $\operatorname{PE}(34: 2)$ & 715.5 & 10.6 & C39H74NO8P & $\begin{array}{l}\text { Glyceroph } \\
\text { ospholipid } \\
\text { s }\end{array}$ & $\begin{array}{l}\text { Glyceroph } \\
\text { osphoetha } \\
\text { nolamines }\end{array}$ & $\begin{array}{l}28833 \\
.8\end{array}$ & $\begin{array}{l}51013 . \\
7\end{array}$ & $\begin{array}{l}99853 . \\
7\end{array}$ & $\begin{array}{l}100633 \\
.8\end{array}$ & $\begin{array}{l}110778 \\
.6\end{array}$ & $\begin{array}{l}125490 \\
.9\end{array}$ & 0.0 & 0.0 & 0.0 & 0.0 & 0.0 & 0.0 \\
\hline $\operatorname{PE}(36: 2)$ & 743.5 & 12.9 & C41H78NO8P & $\begin{array}{l}\text { Glyceroph } \\
\text { ospholipid } \\
\text { s }\end{array}$ & $\begin{array}{l}\text { Glyceroph } \\
\text { osphoetha } \\
\text { nolamines }\end{array}$ & $\begin{array}{l}12120 \\
38.9\end{array}$ & $\begin{array}{l}199905 \\
5.5\end{array}$ & $\begin{array}{l}256243 \\
5.8\end{array}$ & $\begin{array}{l}280332 \\
9.5\end{array}$ & $\begin{array}{l}396369 \\
3.8\end{array}$ & $\begin{array}{l}303410 \\
0.0\end{array}$ & $\begin{array}{l}14137 . \\
2\end{array}$ & 0.0 & 0.0 & 0.0 & 0.0 & 0.0 \\
\hline $\operatorname{PE}(36: 3)$ & 741.5 & 12.2 & C41H76NO8P & $\begin{array}{l}\text { Glyceroph } \\
\text { ospholipid } \\
\text { s }\end{array}$ & $\begin{array}{l}\text { Glyceroph } \\
\text { osphoetha } \\
\text { nolamines }\end{array}$ & $\begin{array}{l}13701 \\
6.2\end{array}$ & $\begin{array}{l}190131 \\
.0\end{array}$ & $\begin{array}{l}253870 \\
.8\end{array}$ & $\begin{array}{l}271342 \\
.0\end{array}$ & $\begin{array}{l}208881 \\
.6\end{array}$ & $\begin{array}{l}293959 \\
.8\end{array}$ & 0.0 & 0.0 & 0.0 & 0.0 & 0.0 & 0.0 \\
\hline $\operatorname{PE}(36: 4)$ & 739.5 & 11.9 & C41H74NO8P & $\begin{array}{l}\text { Glyceroph } \\
\text { ospholipid } \\
\text { s }\end{array}$ & $\begin{array}{l}\text { Glyceroph } \\
\text { osphoetha } \\
\text { nolamines }\end{array}$ & $\begin{array}{l}10009 \\
2.4\end{array}$ & $\begin{array}{l}180739 \\
.0\end{array}$ & $\begin{array}{l}191365 \\
.8\end{array}$ & $\begin{array}{l}221853 \\
.6\end{array}$ & $\begin{array}{l}181730 \\
.8\end{array}$ & $\begin{array}{l}259891 \\
.1\end{array}$ & 0.0 & 0.0 & 0.0 & 0.0 & 0.0 & 0.0 \\
\hline $\operatorname{PE}(36: 5)$ & 737.5 & 11.6 & $\mathrm{C} 41 \mathrm{H} 72 \mathrm{NO} 8 \mathrm{P}$ & $\begin{array}{l}\text { Glyceroph } \\
\text { ospholipid } \\
\text { s }\end{array}$ & $\begin{array}{l}\text { Glyceroph } \\
\text { osphoetha } \\
\text { nolamines }\end{array}$ & $\begin{array}{l}10402 \\
.3\end{array}$ & $\begin{array}{l}15628 \\
4\end{array}$ & $\begin{array}{l}14347 . \\
9\end{array}$ & $\begin{array}{l}17070 . \\
9\end{array}$ & $\begin{array}{l}12800 \\
7\end{array}$ & $\begin{array}{l}24237 . \\
3\end{array}$ & 0.0 & 0.0 & 0.0 & 0.0 & 0.0 & 0.0 \\
\hline $\operatorname{PE}(38: 0)$ & 775.6 & 15.0 & C43H86NO8P & $\begin{array}{l}\text { Glyceroph } \\
\text { ospholipid } \\
\text { s }\end{array}$ & $\begin{array}{l}\text { Glyceroph } \\
\text { osphoetha } \\
\text { nolamines }\end{array}$ & $\begin{array}{l}14714 \\
0.0\end{array}$ & $\begin{array}{l}256366 \\
.9\end{array}$ & $\begin{array}{l}336087 \\
.4\end{array}$ & $\begin{array}{l}343623 \\
.7\end{array}$ & $\begin{array}{l}210560 \\
.6\end{array}$ & $\begin{array}{l}381840 \\
.9\end{array}$ & 0.0 & 0.0 & 0.0 & 0.0 & 0.0 & 0.0 \\
\hline $\operatorname{PE}(38: 1)$ & 773.6 & 14.9 & C43H84NO8P & $\begin{array}{l}\text { Glyceroph } \\
\text { ospholipid } \\
\text { s }\end{array}$ & $\begin{array}{l}\text { Glyceroph } \\
\text { osphoetha } \\
\text { nolamines }\end{array}$ & $\begin{array}{l}14816 \\
8.3\end{array}$ & $\begin{array}{l}231567 \\
.8\end{array}$ & $\begin{array}{l}289144 \\
.4\end{array}$ & $\begin{array}{l}267396 \\
.9\end{array}$ & $\begin{array}{l}156806 \\
.1\end{array}$ & $\begin{array}{l}285023 \\
.9\end{array}$ & 0.0 & 0.0 & 0.0 & 0.0 & 0.0 & 0.0 \\
\hline $\operatorname{PE}(38: 2)$ & 771.6 & 13.3 & C43H82NO8P & $\begin{array}{l}\text { Glyceroph } \\
\text { ospholipid } \\
\text { s }\end{array}$ & $\begin{array}{l}\text { Glyceroph } \\
\text { osphoetha } \\
\text { nolamines }\end{array}$ & $\begin{array}{l}12717 \\
5.2\end{array}$ & $\begin{array}{l}139469 \\
.7\end{array}$ & $\begin{array}{l}149586 \\
.6\end{array}$ & $\begin{array}{l}134420 \\
.0\end{array}$ & $\begin{array}{l}103369 \\
.8\end{array}$ & $\begin{array}{l}134384 \\
.5\end{array}$ & 0.0 & 0.0 & 0.0 & 0.0 & 0.0 & 0.0 \\
\hline $\operatorname{PE}(38: 3)$ & 769.6 & 12.9 & C43H80NO8P & $\begin{array}{l}\text { Glyceroph } \\
\text { ospholipid } \\
\text { s }\end{array}$ & $\begin{array}{l}\text { Glyceroph } \\
\text { osphoetha } \\
\text { nolamines }\end{array}$ & $\begin{array}{l}10445 \\
49.5\end{array}$ & $\begin{array}{l}133511 \\
2.8\end{array}$ & $\begin{array}{l}157726 \\
7.3\end{array}$ & $\begin{array}{l}158929 \\
6.4\end{array}$ & $\begin{array}{l}105331 \\
4.6\end{array}$ & $\begin{array}{l}194518 \\
9.5\end{array}$ & $\begin{array}{l}11276 . \\
8\end{array}$ & 0.0 & 0.0 & 0.0 & 0.0 & 0.0 \\
\hline $\operatorname{PE}(38: 4)$ & 767.5 & 13.2 & $\mathrm{C} 43 \mathrm{H} 78 \mathrm{NO} 8 \mathrm{P}$ & $\begin{array}{l}\text { Glyceroph } \\
\text { ospholipid } \\
\text { s }\end{array}$ & $\begin{array}{l}\text { Glyceroph } \\
\text { osphoetha } \\
\text { nolamines }\end{array}$ & $\begin{array}{l}20930 \\
36.1\end{array}$ & $\begin{array}{l}300762 \\
2.8\end{array}$ & $\begin{array}{l}317004 \\
9.3\end{array}$ & $\begin{array}{l}310520 \\
8.3\end{array}$ & $\begin{array}{l}206398 \\
3.0\end{array}$ & $\begin{array}{l}402783 \\
8.3\end{array}$ & $\begin{array}{l}22195 . \\
9\end{array}$ & 0.0 & 0.0 & 0.0 & 0.0 & 0.0 \\
\hline $\operatorname{PE}(38: 5)$ & 765.5 & 12.5 & $\mathrm{C} 43 \mathrm{H} 76 \mathrm{NO} 8 \mathrm{P}$ & $\begin{array}{l}\text { Glyceroph } \\
\text { ospholipid } \\
\text { s }\end{array}$ & $\begin{array}{l}\text { Glyceroph } \\
\text { osphoetha } \\
\text { nolamines }\end{array}$ & $\begin{array}{l}21033 \\
2.0\end{array}$ & $\begin{array}{l}207840 \\
.3\end{array}$ & $\begin{array}{l}241986 \\
.6\end{array}$ & $\begin{array}{l}246100 \\
.1\end{array}$ & $\begin{array}{l}138771 \\
.2\end{array}$ & $\begin{array}{l}283283 \\
.1\end{array}$ & 0.0 & 0.0 & 0.0 & 0.0 & 0.0 & 0.0 \\
\hline $\operatorname{PE}(38: 6)$ & 763.5 & 11.7 & C43H74NO8P & $\begin{array}{l}\text { Glyceroph } \\
\text { ospholipid } \\
\text { s }\end{array}$ & $\begin{array}{l}\text { Glyceroph } \\
\text { osphoetha } \\
\text { nolamines }\end{array}$ & $\begin{array}{l}25295 \\
.0\end{array}$ & $\begin{array}{l}50280 . \\
9\end{array}$ & $\begin{array}{l}40534 . \\
3\end{array}$ & $\begin{array}{l}53251 . \\
9\end{array}$ & $\begin{array}{l}44268 . \\
9\end{array}$ & $\begin{array}{l}87775 . \\
3\end{array}$ & 0.0 & 0.0 & 0.0 & 0.0 & 0.0 & 0.0 \\
\hline $\operatorname{PE}(40: 1)$ & 801.6 & 15.6 & C45H88NO8P & $\begin{array}{l}\text { Glyceroph } \\
\text { ospholipid } \\
\text { s }\end{array}$ & $\begin{array}{l}\text { Glyceroph } \\
\text { osphoetha } \\
\text { nolamines }\end{array}$ & $\begin{array}{l}18422 \\
1.4\end{array}$ & $\begin{array}{l}278767 \\
.2\end{array}$ & $\begin{array}{l}447366 \\
.5\end{array}$ & $\begin{array}{l}471664 \\
.8\end{array}$ & $\begin{array}{l}360997 \\
.0\end{array}$ & $\begin{array}{l}400068 \\
.4\end{array}$ & $\begin{array}{l}31140 . \\
7\end{array}$ & $\begin{array}{l}25109 . \\
1\end{array}$ & $\begin{array}{l}28448 . \\
1\end{array}$ & $\begin{array}{l}26559 . \\
6\end{array}$ & $\begin{array}{l}24737 . \\
4\end{array}$ & $\begin{array}{l}24283 . \\
3\end{array}$ \\
\hline
\end{tabular}




\begin{tabular}{|c|c|c|c|c|c|c|c|c|c|c|c|c|c|c|c|c|c|}
\hline $\operatorname{PE}(40: 10)$ & 783.5 & 9.3 & $\mathrm{C} 45 \mathrm{H} 70 \mathrm{NO} 8 \mathrm{P}$ & $\begin{array}{l}\text { Glyceroph } \\
\text { ospholipid } \\
\text { s }\end{array}$ & $\begin{array}{l}\text { Glyceroph } \\
\text { osphoetha } \\
\text { nolamines }\end{array}$ & $\begin{array}{l}30307 \\
.5\end{array}$ & 7486.5 & 5653.2 & 0.0 & 0.0 & 0.0 & 0.0 & 0.0 & 0.0 & 0.0 & 0.0 & 0.0 \\
\hline $\operatorname{PE}(40: 2)$ & 799.6 & 14.5 & C45H86NO8P & $\begin{array}{l}\text { Glyceroph } \\
\text { ospholipid } \\
\text { s }\end{array}$ & $\begin{array}{l}\text { Glyceroph } \\
\text { osphoetha } \\
\text { nolamines }\end{array}$ & $\begin{array}{l}86459 \\
8.9\end{array}$ & $\begin{array}{l}128850 \\
7.6\end{array}$ & $\begin{array}{l}210344 \\
1.8\end{array}$ & $\begin{array}{l}264252 \\
8.5\end{array}$ & $\begin{array}{l}268521 \\
5.8\end{array}$ & $\begin{array}{l}202174 \\
8.5\end{array}$ & 0.0 & 0.0 & 0.0 & 0.0 & 0.0 & 0.0 \\
\hline $\operatorname{PE}(40: 5)$ & 793.6 & 12.9 & C45H80NO8P & $\begin{array}{l}\text { Glyceroph } \\
\text { ospholipid } \\
\text { s }\end{array}$ & $\begin{array}{l}\text { Glyceroph } \\
\text { osphoetha } \\
\text { nolamines }\end{array}$ & $\begin{array}{l}19146 \\
8.0\end{array}$ & $\begin{array}{l}348363 \\
.0\end{array}$ & $\begin{array}{l}494670 \\
.5\end{array}$ & $\begin{array}{l}452258 \\
.2\end{array}$ & $\begin{array}{l}202756 \\
.6\end{array}$ & $\begin{array}{l}688275 \\
.3\end{array}$ & 0.0 & 0.0 & 0.0 & 0.0 & 0.0 & 0.0 \\
\hline $\operatorname{PE}(40: 6)$ & 791.5 & 12.2 & $\mathrm{C} 45 \mathrm{H} 78 \mathrm{NO} 8 \mathrm{P}$ & $\begin{array}{l}\text { Glyceroph } \\
\text { ospholipid } \\
\text { s }\end{array}$ & $\begin{array}{l}\text { Glyceroph } \\
\text { osphoetha } \\
\text { nolamines }\end{array}$ & $\begin{array}{l}26181 \\
8.3\end{array}$ & $\begin{array}{l}455280 \\
.6\end{array}$ & $\begin{array}{l}668971 \\
.0\end{array}$ & $\begin{array}{l}837732 \\
.0\end{array}$ & $\begin{array}{l}732936 \\
.7\end{array}$ & $\begin{array}{l}847157 \\
.1\end{array}$ & 0.0 & 0.0 & 0.0 & 0.0 & 0.0 & 0.0 \\
\hline PE(40:8) & 787.5 & 12.6 & $\mathrm{C} 45 \mathrm{H} 74 \mathrm{NO} 8 \mathrm{P}$ & $\begin{array}{l}\text { Glyceroph } \\
\text { ospholipid } \\
\text { s }\end{array}$ & $\begin{array}{l}\text { Glyceroph } \\
\text { osphoetha } \\
\text { nolamines }\end{array}$ & $\begin{array}{l}11112 \\
.3\end{array}$ & 0.0 & 0.0 & 0.0 & 0.0 & 0.0 & 0.0 & 0.0 & 0.0 & 0.0 & 0.0 & 0.0 \\
\hline PE(40:9) & 785.5 & 9.7 & $\mathrm{C} 45 \mathrm{H} 72 \mathrm{NO} 8 \mathrm{P}$ & $\begin{array}{l}\text { Glyceroph } \\
\text { ospholipid } \\
\text { s }\end{array}$ & $\begin{array}{l}\text { Glyceroph } \\
\text { osphoetha } \\
\text { nolamines }\end{array}$ & $\begin{array}{l}55697 \\
.9\end{array}$ & $\begin{array}{l}13734 . \\
5\end{array}$ & 6256.7 & 0.0 & 0.0 & 0.0 & 0.0 & 0.0 & 0.0 & 0.0 & 0.0 & 0.0 \\
\hline $\mathrm{PE}(42: 2)$ & 827.6 & 16.1 & C47H90NO8P & $\begin{array}{l}\text { Glyceroph } \\
\text { ospholipid } \\
\text { s }\end{array}$ & $\begin{array}{l}\text { Glyceroph } \\
\text { osphoetha } \\
\text { nolamines }\end{array}$ & $\begin{array}{l}33044 \\
.2\end{array}$ & $\begin{array}{l}48785 . \\
5\end{array}$ & $\begin{array}{l}73064 . \\
6\end{array}$ & $\begin{array}{l}80051 . \\
1\end{array}$ & $\begin{array}{l}62405 . \\
5\end{array}$ & $\begin{array}{l}77533 . \\
5\end{array}$ & $\begin{array}{l}16517 . \\
0\end{array}$ & $\begin{array}{l}16610 . \\
1\end{array}$ & $\begin{array}{l}18512 . \\
7\end{array}$ & $\begin{array}{l}12071 . \\
8\end{array}$ & $\begin{array}{l}17779 . \\
2\end{array}$ & $\begin{array}{l}15705 . \\
3\end{array}$ \\
\hline $\operatorname{PE}(42: 3)$ & 825.6 & 15.3 & C47H88NO8P & $\begin{array}{l}\text { Glyceroph } \\
\text { ospholipid } \\
\text { s }\end{array}$ & $\begin{array}{l}\text { Glyceroph } \\
\text { osphoetha } \\
\text { nolamines }\end{array}$ & $\begin{array}{l}17226 \\
6.8\end{array}$ & $\begin{array}{l}273314 \\
.7\end{array}$ & $\begin{array}{l}434301 \\
.7\end{array}$ & $\begin{array}{l}479573 \\
.4\end{array}$ & $\begin{array}{l}292194 \\
.7\end{array}$ & $\begin{array}{l}449645 \\
.7\end{array}$ & 0.0 & 0.0 & 0.0 & 0.0 & 0.0 & 0.0 \\
\hline $\operatorname{PE}(42: 6)$ & 819.6 & 13.3 & C47H82NO8P & $\begin{array}{l}\text { Glyceroph } \\
\text { ospholipid } \\
\text { s }\end{array}$ & $\begin{array}{l}\text { Glyceroph } \\
\text { osphoetha } \\
\text { nolamines }\end{array}$ & $\begin{array}{l}24206 \\
2.2\end{array}$ & $\begin{array}{l}379898 \\
.2\end{array}$ & $\begin{array}{l}459518 \\
.0\end{array}$ & $\begin{array}{l}475196 \\
.5\end{array}$ & $\begin{array}{l}403716 \\
.2\end{array}$ & $\begin{array}{l}510080 \\
.1\end{array}$ & 0.0 & 0.0 & 0.0 & 0.0 & 0.0 & 0.0 \\
\hline $\operatorname{PE}(42: 7)$ & 817.6 & 12.3 & C47H80NO8P & $\begin{array}{l}\text { Glyceroph } \\
\text { ospholipid } \\
\text { s }\end{array}$ & $\begin{array}{l}\text { Glyceroph } \\
\text { osphoetha } \\
\text { nolamines }\end{array}$ & $\begin{array}{l}90417 \\
.5\end{array}$ & $\begin{array}{l}149382 \\
.9\end{array}$ & $\begin{array}{l}286672 \\
.2\end{array}$ & $\begin{array}{l}328156 \\
.1\end{array}$ & $\begin{array}{l}221014 \\
.7\end{array}$ & $\begin{array}{l}334167 \\
.6\end{array}$ & 0.0 & 0.0 & 0.0 & 0.0 & 0.0 & 0.0 \\
\hline $\operatorname{PE}(44: 2)$ & 855.7 & 19.6 & C49H94NO8P & $\begin{array}{l}\text { Glyceroph } \\
\text { ospholipid } \\
\text { s }\end{array}$ & $\begin{array}{l}\text { Glyceroph } \\
\text { osphoetha } \\
\text { nolamines }\end{array}$ & 0.0 & 0.0 & 0.0 & 8330.8 & 0.0 & 0.0 & 0.0 & 0.0 & 0.0 & 0.0 & 0.0 & 0.0 \\
\hline PE(44:4) & 851.6 & 16.7 & C49H90NO8P & $\begin{array}{l}\text { Glyceroph } \\
\text { ospholipid } \\
\text { s }\end{array}$ & $\begin{array}{l}\text { Glyceroph } \\
\text { osphoetha } \\
\text { nolamines }\end{array}$ & $\begin{array}{l}41910 \\
.3\end{array}$ & $\begin{array}{l}74698 . \\
7\end{array}$ & $\begin{array}{l}101108 \\
.0\end{array}$ & $\begin{array}{l}109275 \\
.4\end{array}$ & $\begin{array}{l}43320 . \\
4\end{array}$ & $\begin{array}{l}115819 \\
.0\end{array}$ & 0.0 & 0.0 & 0.0 & 0.0 & 0.0 & 0.0 \\
\hline $\operatorname{PE}(46: 1)$ & 885.7 & 21.2 & $\begin{array}{l}\text { C51H100NO8 } \\
\text { P }\end{array}$ & $\begin{array}{l}\text { Glyceroph } \\
\text { ospholipid } \\
\text { s }\end{array}$ & $\begin{array}{l}\text { Glyceroph } \\
\text { osphoetha } \\
\text { nolamines }\end{array}$ & 0.0 & 0.0 & $\begin{array}{l}12641 . \\
6\end{array}$ & 0.0 & 0.0 & $\begin{array}{l}12861 . \\
9\end{array}$ & 0.0 & 0.0 & 0.0 & 0.0 & 0.0 & 0.0 \\
\hline
\end{tabular}




\begin{tabular}{|c|c|c|c|c|c|c|c|c|c|c|c|c|c|c|c|c|c|}
\hline $\operatorname{PE}(46: 3)$ & 881.7 & 19.7 & C51H96NO8P & $\begin{array}{l}\text { Glyceroph } \\
\text { ospholipid } \\
\mathrm{s}\end{array}$ & $\begin{array}{l}\text { Glyceroph } \\
\text { osphoetha } \\
\text { nolamines }\end{array}$ & $\begin{array}{l}7133 . \\
2\end{array}$ & $\begin{array}{l}11004 . \\
5\end{array}$ & $\begin{array}{l}13761 . \\
7\end{array}$ & $\begin{array}{l}15166 . \\
8\end{array}$ & 0.0 & $\begin{array}{l}14053 . \\
6\end{array}$ & 0.0 & 0.0 & 0.0 & 0.0 & 0.0 & 0.0 \\
\hline $\operatorname{PE}(46: 4)$ & 879.7 & 18.7 & C51H94NO8P & $\begin{array}{l}\text { Glyceroph } \\
\text { ospholipid } \\
\text { s }\end{array}$ & $\begin{array}{l}\text { Glyceroph } \\
\text { osphoetha } \\
\text { nolamines }\end{array}$ & $\begin{array}{l}9666 . \\
9\end{array}$ & $\begin{array}{l}12211 . \\
7\end{array}$ & $\begin{array}{l}18988 . \\
2\end{array}$ & $\begin{array}{l}15896 . \\
6\end{array}$ & 3584.0 & $\begin{array}{l}19029 . \\
1\end{array}$ & 0.0 & 0.0 & 0.0 & 0.0 & 0.0 & 0.0 \\
\hline $\operatorname{PE}(48: 1)$ & 913.8 & 21.4 & $\begin{array}{l}\text { C53H104NO8 } \\
\text { P }\end{array}$ & $\begin{array}{l}\text { Glyceroph } \\
\text { ospholipid } \\
\mathrm{s}\end{array}$ & $\begin{array}{l}\text { Glyceroph } \\
\text { osphoetha } \\
\text { nolamines }\end{array}$ & $\begin{array}{l}13645 \\
.5\end{array}$ & 0.0 & 5413.3 & 0.0 & 0.0 & 0.0 & 0.0 & 0.0 & 0.0 & 0.0 & 0.0 & 0.0 \\
\hline $\mathrm{PE}(\mathrm{O}-16: 0 / 13: 0)$ & 635.5 & 11.3 & C34H70NO7P & $\begin{array}{l}\text { Glyceroph } \\
\text { ospholipid } \\
\mathrm{s}\end{array}$ & $\begin{array}{l}\text { Glyceroph } \\
\text { osphoetha } \\
\text { nolamines }\end{array}$ & $\begin{array}{l}10846 \\
.3\end{array}$ & 7304.3 & 9306.5 & $\begin{array}{l}10771 . \\
0\end{array}$ & 8140.2 & 9793.7 & 0.0 & 0.0 & 0.0 & 0.0 & 0.0 & 0.0 \\
\hline PE(O-20:0/0:0) & 495.4 & 7.7 & C25H54NO6P & $\begin{array}{l}\text { Glyceroph } \\
\text { ospholipid } \\
\mathrm{s}\end{array}$ & $\begin{array}{l}\text { Glyceroph } \\
\text { osphoetha } \\
\text { nolamines }\end{array}$ & 0.0 & 4817.3 & 3308.6 & 2891.7 & $\begin{array}{l}12613 . \\
4\end{array}$ & 4989.2 & 0.0 & 0.0 & 0.0 & 0.0 & 0.0 & 0.0 \\
\hline $\mathrm{PE}(\mathrm{O}-30: 0)$ & 649.5 & 11.8 & $\mathrm{C} 35 \mathrm{H} 72 \mathrm{NO} 7 \mathrm{P}$ & $\begin{array}{l}\text { Glyceroph } \\
\text { ospholipid } \\
\mathrm{s}\end{array}$ & $\begin{array}{l}\text { Glyceroph } \\
\text { osphoetha } \\
\text { nolamines }\end{array}$ & $\begin{array}{l}38958 \\
.9\end{array}$ & $\begin{array}{l}12059 . \\
7\end{array}$ & 5893.4 & 0.0 & 4561.6 & 0.0 & 0.0 & 0.0 & 0.0 & 0.0 & 0.0 & 0.0 \\
\hline $\mathrm{PE}(\mathrm{O}-36: 0)$ & 733.6 & 15.2 & C41H84NO7P & $\begin{array}{l}\text { Glyceroph } \\
\text { ospholipid } \\
\mathrm{s}\end{array}$ & $\begin{array}{l}\text { Glyceroph } \\
\text { osphoetha } \\
\text { nolamines }\end{array}$ & $\begin{array}{l}54926 \\
.8\end{array}$ & $\begin{array}{l}85514 . \\
0\end{array}$ & $\begin{array}{l}147963 \\
.0\end{array}$ & $\begin{array}{l}175287 \\
.5\end{array}$ & $\begin{array}{l}90263 . \\
4\end{array}$ & $\begin{array}{l}85381 . \\
3\end{array}$ & 0.0 & 0.0 & 0.0 & 0.0 & 0.0 & 0.0 \\
\hline $\mathrm{PE}(\mathrm{O}-38: 5)$ & 751.6 & 13.8 & $\mathrm{C} 43 \mathrm{H} 78 \mathrm{NO} 7 \mathrm{P}$ & $\begin{array}{l}\text { Glyceroph } \\
\text { ospholipid } \\
\mathrm{s}\end{array}$ & $\begin{array}{l}\text { Glyceroph } \\
\text { osphoetha } \\
\text { nolamines }\end{array}$ & $\begin{array}{l}22164 \\
61.3\end{array}$ & $\begin{array}{l}343713 \\
1.8\end{array}$ & $\begin{array}{l}629482 \\
5.0\end{array}$ & $\begin{array}{l}669788 \\
3.0\end{array}$ & $\begin{array}{l}329301 \\
4.8\end{array}$ & $\begin{array}{l}724159 \\
0.0\end{array}$ & $\begin{array}{l}11056 . \\
9\end{array}$ & 0.0 & 0.0 & 0.0 & 0.0 & 0.0 \\
\hline $\mathrm{PE}(\mathrm{O}-42: 4)$ & 809.6 & 16.1 & C47H88NO7P & $\begin{array}{l}\text { Glyceroph } \\
\text { ospholipid } \\
\mathrm{s}\end{array}$ & $\begin{array}{l}\text { Glyceroph } \\
\text { osphoetha } \\
\text { nolamines }\end{array}$ & $\begin{array}{l}71357 \\
.4\end{array}$ & $\begin{array}{l}127643 \\
.3\end{array}$ & $\begin{array}{l}177764 \\
.8\end{array}$ & $\begin{array}{l}195430 \\
.4\end{array}$ & $\begin{array}{l}102580 \\
.2\end{array}$ & $\begin{array}{l}185042 \\
.6\end{array}$ & 0.0 & 0.0 & 0.0 & 0.0 & 0.0 & 0.0 \\
\hline PE(P-20:0) & 493.4 & 7.9 & C25H52NO6P & $\begin{array}{l}\text { Glyceroph } \\
\text { ospholipid } \\
\mathrm{s}\end{array}$ & $\begin{array}{l}\text { Glyceroph } \\
\text { osphoetha } \\
\text { nolamines }\end{array}$ & $\begin{array}{l}6862 . \\
7\end{array}$ & 6215.7 & $\begin{array}{l}10389 . \\
8\end{array}$ & $\begin{array}{l}10855 \\
8\end{array}$ & $\begin{array}{l}12352 . \\
8\end{array}$ & 7708.3 & 0.0 & 0.0 & 0.0 & 0.0 & 0.0 & 0.0 \\
\hline $\begin{array}{l}\mathrm{PE}(\mathrm{P}- \\
\text { 20:0/22:4(7Z_10Z_13Z_16Z) } \\
)\end{array}$ & 807.6 & 15.1 & C47H86NO7P & $\begin{array}{l}\text { Glyceroph } \\
\text { ospholipid } \\
\text { s }\end{array}$ & $\begin{array}{l}\text { Glyceroph } \\
\text { osphoetha } \\
\text { nolamines }\end{array}$ & $\begin{array}{l}29321 \\
.1\end{array}$ & $\begin{array}{l}40956 . \\
3\end{array}$ & $\begin{array}{l}56214 . \\
1\end{array}$ & $\begin{array}{l}64292 . \\
8\end{array}$ & $\begin{array}{l}39871 . \\
7\end{array}$ & $\begin{array}{l}64119 . \\
2\end{array}$ & 0.0 & 0.0 & 0.0 & 0.0 & 0.0 & 0.0 \\
\hline $\mathrm{PE}(\mathrm{P}-32: 0)$ & 675.5 & 12.6 & C37H74NO7P & $\begin{array}{l}\text { Glyceroph } \\
\text { ospholipid } \\
\text { s }\end{array}$ & $\begin{array}{l}\text { Glyceroph } \\
\text { osphoetha } \\
\text { nolamines }\end{array}$ & $\begin{array}{l}65286 \\
.5\end{array}$ & $\begin{array}{l}134753 \\
.7\end{array}$ & $\begin{array}{l}210515 \\
.5\end{array}$ & $\begin{array}{l}296437 \\
.2\end{array}$ & $\begin{array}{l}267254 \\
.9\end{array}$ & $\begin{array}{l}211930 \\
.9\end{array}$ & 0.0 & 0.0 & 0.0 & 0.0 & 0.0 & 0.0 \\
\hline PE(P-34:3) & 703.6 & 13.5 & C39H78NO7P & $\begin{array}{l}\text { Glyceroph } \\
\text { ospholipid } \\
\mathrm{s}\end{array}$ & $\begin{array}{l}\text { Glyceroph } \\
\text { osphoetha } \\
\text { nolamines }\end{array}$ & $\begin{array}{l}59372 \\
3.1\end{array}$ & $\begin{array}{l}814126 \\
.6\end{array}$ & $\begin{array}{l}980205 \\
.5\end{array}$ & $\begin{array}{l}110455 \\
8.8\end{array}$ & $\begin{array}{l}102585 \\
0.7\end{array}$ & $\begin{array}{l}940769 \\
.9\end{array}$ & 5808.5 & 0.0 & 0.0 & 0.0 & 0.0 & 0.0 \\
\hline
\end{tabular}




\begin{tabular}{|c|c|c|c|c|c|c|c|c|c|c|c|c|c|c|c|c|}
\hline PE(P-36:0) & 731.6 & 14.3 & $\mathrm{C} 41 \mathrm{H} 82 \mathrm{NO} 7 \mathrm{P}$ & $\begin{array}{l}\text { Glyceroph } \\
\text { ospholipid } \\
\mathrm{s}\end{array}$ & $\begin{array}{l}\text { Glyceroph } \\
\text { osphoetha } \\
\text { nolamines }\end{array}$ & $\begin{array}{l}22985 \\
04.8\end{array}$ & $\begin{array}{l}395843 \\
1.3\end{array}$ & $\begin{array}{l}546310 \\
6.0\end{array}$ & $\begin{array}{l}678275 \\
7.5\end{array}$ & $\begin{array}{l}460346 \\
2.5\end{array}$ & $\begin{array}{l}583869 \\
4.0\end{array}$ & $\begin{array}{l}20298 . \\
9\end{array}$ & 0.0 & 3379.8 & 0.0 & $\begin{array}{l}10286 . \\
6\end{array}$ \\
\hline PE(P-36:4) & 723.5 & 12.5 & $\mathrm{C} 41 \mathrm{H} 74 \mathrm{NO} 7 \mathrm{P}$ & $\begin{array}{l}\text { Glyceroph } \\
\text { ospholipid } \\
\mathrm{s}\end{array}$ & $\begin{array}{l}\text { Glyceroph } \\
\text { osphoetha } \\
\text { nolamines }\end{array}$ & $\begin{array}{l}23490 \\
.5\end{array}$ & $\begin{array}{l}36438 . \\
6\end{array}$ & $\begin{array}{l}57199 . \\
0\end{array}$ & $\begin{array}{l}76919 . \\
3\end{array}$ & $\begin{array}{l}35375 . \\
0\end{array}$ & $\begin{array}{l}94232 . \\
2\end{array}$ & 0.0 & 0.0 & 0.0 & 0.0 & 0.0 \\
\hline PE(P-38:0) & 759.6 & 15.5 & $\mathrm{C} 43 \mathrm{H} 86 \mathrm{NO} 7 \mathrm{P}$ & $\begin{array}{l}\text { Glyceroph } \\
\text { ospholipid } \\
\mathrm{s}\end{array}$ & $\begin{array}{l}\text { Glyceroph } \\
\text { osphoetha } \\
\text { nolamines }\end{array}$ & $\begin{array}{l}24106 \\
0.4\end{array}$ & $\begin{array}{l}421832 \\
.2\end{array}$ & $\begin{array}{l}689922 \\
.1\end{array}$ & $\begin{array}{l}754541 \\
.3\end{array}$ & $\begin{array}{l}408733 \\
.8\end{array}$ & $\begin{array}{l}828949 \\
.6\end{array}$ & 0.0 & 0.0 & 0.0 & 0.0 & 0.0 \\
\hline PE(P-38:1) & 757.6 & 14.7 & $\mathrm{C} 43 \mathrm{H} 84 \mathrm{NO} 7 \mathrm{P}$ & $\begin{array}{l}\text { Glyceroph } \\
\text { ospholipid } \\
\mathrm{s}\end{array}$ & $\begin{array}{l}\text { Glyceroph } \\
\text { osphoetha } \\
\text { nolamines }\end{array}$ & $\begin{array}{l}18039 \\
9.8\end{array}$ & $\begin{array}{l}302988 \\
.5\end{array}$ & $\begin{array}{l}360386 \\
.4\end{array}$ & $\begin{array}{l}342607 \\
.9\end{array}$ & $\begin{array}{l}183413 \\
.8\end{array}$ & $\begin{array}{l}348697 \\
.7\end{array}$ & 0.0 & 0.0 & 0.0 & 0.0 & 0.0 \\
\hline PE(P-38:3) & 753.6 & 14.0 & $\mathrm{C} 43 \mathrm{H} 80 \mathrm{NO} 7 \mathrm{P}$ & $\begin{array}{l}\text { Glyceroph } \\
\text { ospholipid } \\
\text { s }\end{array}$ & $\begin{array}{l}\text { Glyceroph } \\
\text { osphoetha } \\
\text { nolamines }\end{array}$ & $\begin{array}{l}16579 \\
79.6\end{array}$ & $\begin{array}{l}207570 \\
3.8\end{array}$ & $\begin{array}{l}287937 \\
7.0\end{array}$ & $\begin{array}{l}294093 \\
5.0\end{array}$ & $\begin{array}{l}196359 \\
2.4\end{array}$ & $\begin{array}{l}308789 \\
3.8\end{array}$ & 0.0 & 0.0 & 0.0 & 0.0 & 0.0 \\
\hline PE(P-38:5) & 749.5 & 13.0 & $\mathrm{C} 43 \mathrm{H} 76 \mathrm{NO} 7 \mathrm{P}$ & $\begin{array}{l}\text { Glyceroph } \\
\text { ospholipid } \\
\text { s }\end{array}$ & $\begin{array}{l}\text { Glyceroph } \\
\text { osphoetha } \\
\text { nolamines }\end{array}$ & $\begin{array}{l}20262 \\
8.3\end{array}$ & $\begin{array}{l}134568 \\
.9\end{array}$ & $\begin{array}{l}127259 \\
.0\end{array}$ & $\begin{array}{l}130903 \\
.2\end{array}$ & $\begin{array}{l}57923 . \\
4\end{array}$ & $\begin{array}{l}135421 \\
.1\end{array}$ & 0.0 & 0.0 & 0.0 & 0.0 & 0.0 \\
\hline PE(P-40:0) & 787.6 & 16.8 & $\mathrm{C} 45 \mathrm{H} 90 \mathrm{NO} 7 \mathrm{P}$ & $\begin{array}{l}\text { Glyceroph } \\
\text { ospholipid } \\
\mathrm{s}\end{array}$ & $\begin{array}{l}\text { Glyceroph } \\
\text { osphoetha } \\
\text { nolamines }\end{array}$ & $\begin{array}{l}56749 \\
.9\end{array}$ & $\begin{array}{l}94041 . \\
0\end{array}$ & $\begin{array}{l}137876 \\
.3\end{array}$ & $\begin{array}{l}159099 \\
.5\end{array}$ & $\begin{array}{l}122273 \\
.4\end{array}$ & $\begin{array}{l}149054 \\
.8\end{array}$ & 0.0 & 0.0 & 0.0 & 0.0 & 0.0 \\
\hline PE(P-40:1) & 785.6 & 16.0 & C45H88NO7P & $\begin{array}{l}\text { Glyceroph } \\
\text { ospholipid } \\
\text { s }\end{array}$ & $\begin{array}{l}\text { Glyceroph } \\
\text { osphoetha } \\
\text { nolamines }\end{array}$ & $\begin{array}{l}37803 \\
.9\end{array}$ & $\begin{array}{l}57660 . \\
8\end{array}$ & $\begin{array}{l}59548 . \\
5\end{array}$ & $\begin{array}{l}61929 . \\
0\end{array}$ & $\begin{array}{l}63153 . \\
5\end{array}$ & $\begin{array}{l}83628 . \\
0\end{array}$ & 0.0 & 0.0 & 0.0 & 0.0 & 0.0 \\
\hline $\mathrm{PE}(\mathrm{P}-40: 2)$ & 783.6 & 15.1 & $\mathrm{C} 45 \mathrm{H} 86 \mathrm{NO} 7 \mathrm{P}$ & $\begin{array}{l}\text { Glyceroph } \\
\text { ospholipid } \\
\text { s }\end{array}$ & $\begin{array}{l}\text { Glyceroph } \\
\text { osphoetha } \\
\text { nolamines }\end{array}$ & $\begin{array}{l}48600 \\
.3\end{array}$ & $\begin{array}{l}85932 . \\
1\end{array}$ & $\begin{array}{l}135586 \\
.8\end{array}$ & $\begin{array}{l}152166 \\
.5\end{array}$ & $\begin{array}{l}102619 \\
.7\end{array}$ & $\begin{array}{l}146040 \\
.9\end{array}$ & 0.0 & 0.0 & 0.0 & 0.0 & 0.0 \\
\hline Pelargonidin 3-gentiotrioside & 757.2 & 1.2 & $\mathrm{C} 33 \mathrm{H} 41 \mathrm{O} 20$ & $\begin{array}{l}\text { Polyketid } \\
\text { es }\end{array}$ & Flavonoids & $\begin{array}{l}20373 \\
9.4\end{array}$ & $\begin{array}{l}138550 \\
.4\end{array}$ & $\begin{array}{l}79573 . \\
2\end{array}$ & $\begin{array}{l}276839 \\
.0\end{array}$ & 0.0 & $\begin{array}{l}415232 \\
.0\end{array}$ & 0.0 & 0.0 & 0.0 & 0.0 & 0.0 \\
\hline Pentacosene & 350.4 & 16.5 & $\mathrm{C} 25 \mathrm{H} 50$ & $\begin{array}{l}\text { Fatty } \\
\text { Acyls }\end{array}$ & $\begin{array}{l}\text { Hydrocarb } \\
\text { ons }\end{array}$ & $\begin{array}{l}31333 \\
5.8\end{array}$ & $\begin{array}{l}104693 \\
.2\end{array}$ & $\begin{array}{l}370060 \\
.1\end{array}$ & $\begin{array}{l}421516 \\
.8\end{array}$ & $\begin{array}{l}225682 \\
.9\end{array}$ & $\begin{array}{l}122817 \\
0.9\end{array}$ & 0.0 & 0.0 & 0.0 & 0.0 & 0.0 \\
\hline $\mathrm{PG}(31: 0)$ & 725.5 & 9.2 & $\mathrm{C} 37 \mathrm{H} 73 \mathrm{O} 10 \mathrm{P}$ & $\begin{array}{l}\text { Glyceroph } \\
\text { ospholipid } \\
\mathrm{s}\end{array}$ & $\begin{array}{l}\text { Glyceroph } \\
\text { osphoglyc } \\
\text { erols }\end{array}$ & $\begin{array}{l}39299 \\
.1\end{array}$ & $\begin{array}{l}60756 . \\
0\end{array}$ & $\begin{array}{l}83602 . \\
0\end{array}$ & $\begin{array}{l}89537 . \\
3\end{array}$ & $\begin{array}{l}119166 \\
.5\end{array}$ & $\begin{array}{l}54297 . \\
1\end{array}$ & 0.0 & 0.0 & 0.0 & 0.0 & 0.0 \\
\hline $\mathrm{PG}(32: 0)$ & 739.5 & 9.8 & $\mathrm{C} 38 \mathrm{H} 75 \mathrm{O} 10 \mathrm{P}$ & $\begin{array}{l}\text { Glyceroph } \\
\text { ospholipid } \\
\text { s }\end{array}$ & $\begin{array}{l}\text { Glyceroph } \\
\text { osphoglyc } \\
\text { erols }\end{array}$ & $\begin{array}{l}33203 \\
.4\end{array}$ & $\begin{array}{l}73404 . \\
6\end{array}$ & $\begin{array}{l}94212 . \\
7\end{array}$ & $\begin{array}{l}113597 \\
.6\end{array}$ & $\begin{array}{l}106556 \\
.7\end{array}$ & $\begin{array}{l}70402 . \\
7\end{array}$ & 0.0 & 0.0 & 0.0 & 0.0 & 0.0 \\
\hline
\end{tabular}




\begin{tabular}{|c|c|c|c|c|c|c|c|c|c|c|c|c|c|c|c|c|c|}
\hline $\mathrm{PG}(34: 1)$ & 765.6 & 10.1 & $\mathrm{C} 40 \mathrm{H} 77 \mathrm{O} 10 \mathrm{P}$ & $\begin{array}{l}\text { Glyceroph } \\
\text { ospholipid } \\
\mathrm{s}\end{array}$ & $\begin{array}{l}\text { Glyceroph } \\
\text { osphoglyc } \\
\text { erols }\end{array}$ & $\begin{array}{l}89056 \\
.3\end{array}$ & $\begin{array}{l}114146 \\
.9\end{array}$ & $\begin{array}{l}183780 \\
.8\end{array}$ & $\begin{array}{l}175455 \\
.7\end{array}$ & $\begin{array}{l}146704 \\
.0\end{array}$ & $\begin{array}{l}126488 \\
.3\end{array}$ & 0.0 & 0.0 & 0.0 & 0.0 & 0.0 & 0.0 \\
\hline $\mathrm{PG}(36: 1)$ & 776.6 & 11.9 & $\mathrm{C} 42 \mathrm{H} 81 \mathrm{O} 10 \mathrm{P}$ & $\begin{array}{l}\text { Glyceroph } \\
\text { ospholipid } \\
\mathrm{s}\end{array}$ & $\begin{array}{l}\text { Glyceroph } \\
\text { osphoglyc } \\
\text { erols }\end{array}$ & $\begin{array}{l}11265 \\
9.3\end{array}$ & $\begin{array}{l}185428 \\
.0\end{array}$ & $\begin{array}{l}268586 \\
.1\end{array}$ & $\begin{array}{l}252729 \\
.4\end{array}$ & $\begin{array}{l}241758 \\
.0\end{array}$ & $\begin{array}{l}249405 \\
.2\end{array}$ & 0.0 & 0.0 & 0.0 & 0.0 & 0.0 & 0.0 \\
\hline $\begin{array}{l}\text { Phosphodimethylethanolamin } \\
\text { e }\end{array}$ & 169.1 & 1.5 & $\mathrm{C} 4 \mathrm{H} 12 \mathrm{NO} 4 \mathrm{P}$ & $\begin{array}{l}\text { Lipid } \\
\text { Metabolis } \\
\mathrm{m}\end{array}$ & $\begin{array}{l}\text { Glyceroph } \\
\text { ospholipid } \\
\text { metabolis } \\
\mathrm{m}\end{array}$ & $\begin{array}{l}95647 \\
.7\end{array}$ & $\begin{array}{l}160344 \\
.7\end{array}$ & $\begin{array}{l}115362 \\
.1\end{array}$ & $\begin{array}{l}120596 \\
.1\end{array}$ & 0.0 & $\begin{array}{l}591591 \\
.0\end{array}$ & 0.0 & 0.0 & 0.0 & 0.0 & 0.0 & 0.0 \\
\hline $\operatorname{PI}(31: 0)$ & 796.5 & 10.0 & $\mathrm{C} 40 \mathrm{H} 77 \mathrm{O} 13 \mathrm{P}$ & $\begin{array}{l}\text { Glyceroph } \\
\text { ospholipid } \\
\text { s }\end{array}$ & $\begin{array}{l}\text { Glyceroph } \\
\text { osphoinosi } \\
\text { tols }\end{array}$ & $\begin{array}{l}3849 . \\
3\end{array}$ & 6785.1 & 6694.0 & 7720.7 & $\begin{array}{l}23726 . \\
5\end{array}$ & 9480.1 & 0.0 & 0.0 & 0.0 & 0.0 & 0.0 & 0.0 \\
\hline $\operatorname{PI}(35: 2)$ & 848.5 & 10.4 & C44H81O13P & $\begin{array}{l}\text { Glyceroph } \\
\text { ospholipid } \\
\text { s }\end{array}$ & $\begin{array}{l}\text { Glyceroph } \\
\text { osphoinosi } \\
\text { tols }\end{array}$ & $\begin{array}{l}17559 \\
.0\end{array}$ & $\begin{array}{l}30217 . \\
8\end{array}$ & $\begin{array}{l}32833 \\
6\end{array}$ & $\begin{array}{l}40167 . \\
6\end{array}$ & $\begin{array}{l}89391 . \\
2\end{array}$ & $\begin{array}{l}37713 . \\
1\end{array}$ & 0.0 & 0.0 & 0.0 & 0.0 & 0.0 & 0.0 \\
\hline $\operatorname{PI}(35: 6)$ & 840.5 & 11.8 & $\mathrm{C} 44 \mathrm{H} 73 \mathrm{O} 13 \mathrm{P}$ & $\begin{array}{l}\text { Glyceroph } \\
\text { ospholipid } \\
\text { s }\end{array}$ & $\begin{array}{l}\text { Glyceroph } \\
\text { osphoinosi } \\
\text { tols }\end{array}$ & $\begin{array}{l}17456 \\
7.9\end{array}$ & $\begin{array}{l}312809 \\
.6\end{array}$ & $\begin{array}{l}409730 \\
.6\end{array}$ & $\begin{array}{l}452513 \\
.7\end{array}$ & $\begin{array}{l}577189 \\
.3\end{array}$ & $\begin{array}{l}479299 \\
.7\end{array}$ & 0.0 & 0.0 & 0.0 & 0.0 & 0.0 & 0.0 \\
\hline $\operatorname{PI}(36: 1)$ & 864.6 & 11.4 & C45H85O13P & $\begin{array}{l}\text { Glyceroph } \\
\text { ospholipid } \\
\text { s }\end{array}$ & $\begin{array}{l}\text { Glyceroph } \\
\text { osphoinosi } \\
\text { tols }\end{array}$ & $\begin{array}{l}91886 \\
.4\end{array}$ & $\begin{array}{l}147604 \\
.4\end{array}$ & $\begin{array}{l}197021 \\
.1\end{array}$ & $\begin{array}{l}231991 \\
.9\end{array}$ & $\begin{array}{l}357862 \\
.5\end{array}$ & $\begin{array}{l}173365 \\
.0\end{array}$ & 0.0 & 0.0 & 0.0 & 0.0 & 0.0 & 0.0 \\
\hline $\operatorname{PI}(36: 2)$ & 862.6 & 10.8 & C45H83O13P & $\begin{array}{l}\text { Glyceroph } \\
\text { ospholipid } \\
\text { s }\end{array}$ & $\begin{array}{l}\text { Glyceroph } \\
\text { osphoinosi } \\
\text { tols }\end{array}$ & $\begin{array}{l}67720 \\
.2\end{array}$ & $\begin{array}{l}122622 \\
.6\end{array}$ & $\begin{array}{l}139696 \\
.8\end{array}$ & $\begin{array}{l}169455 \\
.7\end{array}$ & $\begin{array}{l}317541 \\
.7\end{array}$ & $\begin{array}{l}133947 \\
.9\end{array}$ & 0.0 & 0.0 & 0.0 & 0.0 & 0.0 & 0.0 \\
\hline $\operatorname{PI}(36: 3)$ & 860.5 & 10.2 & C45H81O13P & $\begin{array}{l}\text { Glyceroph } \\
\text { ospholipid } \\
\text { s }\end{array}$ & $\begin{array}{l}\text { Glyceroph } \\
\text { osphoinosi } \\
\text { tols }\end{array}$ & $\begin{array}{l}35214 \\
.8\end{array}$ & $\begin{array}{l}57207 . \\
6\end{array}$ & $\begin{array}{l}79510 . \\
9\end{array}$ & $\begin{array}{l}95425 . \\
2\end{array}$ & $\begin{array}{l}150707 \\
.2\end{array}$ & $\begin{array}{l}71736 . \\
3\end{array}$ & 0.0 & 0.0 & 0.0 & 0.0 & 0.0 & 0.0 \\
\hline $\operatorname{PI}(36: 5)$ & 856.5 & 9.7 & C45H77O13P & $\begin{array}{l}\text { Glyceroph } \\
\text { ospholipid } \\
\mathrm{s}\end{array}$ & $\begin{array}{l}\text { Glyceroph } \\
\text { osphoinosi } \\
\text { tols }\end{array}$ & $\begin{array}{l}11936 \\
.3\end{array}$ & $\begin{array}{l}12798 . \\
4\end{array}$ & $\begin{array}{l}10739 . \\
0\end{array}$ & $\begin{array}{l}11751 . \\
1\end{array}$ & $\begin{array}{l}22034 . \\
8\end{array}$ & $\begin{array}{l}10990 . \\
3\end{array}$ & 0.0 & 0.0 & 0.0 & 0.0 & 0.0 & 0.0 \\
\hline $\operatorname{PI}(36: 6)$ & 854.5 & 13.0 & $\mathrm{C} 45 \mathrm{H} 75 \mathrm{O} 13 \mathrm{P}$ & $\begin{array}{l}\text { Glyceroph } \\
\text { ospholipid } \\
\mathrm{s}\end{array}$ & $\begin{array}{l}\text { Glyceroph } \\
\text { osphoinosi } \\
\text { tols }\end{array}$ & $\begin{array}{l}14676 \\
2.4\end{array}$ & $\begin{array}{l}256354 \\
.7\end{array}$ & $\begin{array}{l}420397 \\
.0\end{array}$ & $\begin{array}{l}397250 \\
.6\end{array}$ & $\begin{array}{l}489386 \\
.9\end{array}$ & $\begin{array}{l}405877 \\
.8\end{array}$ & 0.0 & 0.0 & 0.0 & 0.0 & 0.0 & 0.0 \\
\hline $\operatorname{PI}(37: 4)$ & 889.6 & 10.6 & $\begin{array}{l}\mathrm{C} 46 \mathrm{H} 84 \mathrm{NO} 13 \\
\mathrm{P}\end{array}$ & $\begin{array}{l}\text { Glyceroph } \\
\text { ospholipid } \\
\mathrm{s}\end{array}$ & $\begin{array}{l}\text { Glyceroph } \\
\text { osphoinosi } \\
\text { tols }\end{array}$ & $\begin{array}{l}85019 \\
.4\end{array}$ & $\begin{array}{l}140124 \\
.8\end{array}$ & $\begin{array}{l}198590 \\
.6\end{array}$ & $\begin{array}{l}216574 \\
.3\end{array}$ & $\begin{array}{l}254851 \\
.6\end{array}$ & $\begin{array}{l}207218 \\
.1\end{array}$ & 0.0 & 0.0 & 0.0 & 0.0 & 0.0 & 0.0 \\
\hline $\operatorname{PI}(37: 5)$ & 870.5 & 10.1 & $\mathrm{C} 46 \mathrm{H} 79 \mathrm{O} 13 \mathrm{P}$ & $\begin{array}{l}\text { Glyceroph } \\
\text { ospholipid } \\
\text { s }\end{array}$ & $\begin{array}{l}\text { Glyceroph } \\
\text { osphoinosi } \\
\text { tols }\end{array}$ & $\begin{array}{l}47130 \\
.8\end{array}$ & $\begin{array}{l}52462 . \\
8\end{array}$ & $\begin{array}{l}67850 . \\
1\end{array}$ & $\begin{array}{l}72999 . \\
0\end{array}$ & $\begin{array}{l}97600 . \\
8\end{array}$ & $\begin{array}{l}82936 . \\
6\end{array}$ & 0.0 & 0.0 & 0.0 & 0.0 & 0.0 & 0.0 \\
\hline
\end{tabular}




\begin{tabular}{|c|c|c|c|c|c|c|c|c|c|c|c|c|c|c|c|c|c|}
\hline $\operatorname{PI}(38: 0)$ & 911.6 & 11.8 & C47H91O13P & $\begin{array}{l}\text { Glyceroph } \\
\text { ospholipid } \\
\mathrm{s}\end{array}$ & $\begin{array}{l}\text { Glyceroph } \\
\text { osphoinosi } \\
\text { tols }\end{array}$ & $\begin{array}{l}56498 \\
.5\end{array}$ & $\begin{array}{l}79843 . \\
6\end{array}$ & $\begin{array}{l}127666 \\
.4\end{array}$ & $\begin{array}{l}148390 \\
.3\end{array}$ & $\begin{array}{l}196851 \\
.4\end{array}$ & $\begin{array}{l}140343 \\
.6\end{array}$ & 0.0 & 0.0 & 0.0 & 0.0 & 0.0 & 0.0 \\
\hline $\operatorname{PI}(38: 4)$ & 886.6 & 11.1 & C47H83O13P & $\begin{array}{l}\text { Glyceroph } \\
\text { ospholipid } \\
\text { s }\end{array}$ & $\begin{array}{l}\text { Glyceroph } \\
\text { osphoinosi } \\
\text { tols }\end{array}$ & $\begin{array}{l}66977 \\
67.0\end{array}$ & $\begin{array}{l}110734 \\
20.0\end{array}$ & $\begin{array}{l}160867 \\
44.0\end{array}$ & $\begin{array}{l}160314 \\
11.0\end{array}$ & $\begin{array}{l}164024 \\
89.0\end{array}$ & $\begin{array}{l}149536 \\
62.0\end{array}$ & $\begin{array}{l}21945 . \\
5\end{array}$ & 0.0 & 3473.8 & 0.0 & 8849.5 & 0.0 \\
\hline $\operatorname{PI}(38: 5)$ & 884.5 & 10.6 & $\mathrm{C} 47 \mathrm{H} 81 \mathrm{O} 13 \mathrm{P}$ & $\begin{array}{l}\text { Glyceroph } \\
\text { ospholipid } \\
\mathrm{s}\end{array}$ & $\begin{array}{l}\text { Glyceroph } \\
\text { osphoinosi } \\
\text { tols }\end{array}$ & $\begin{array}{l}12843 \\
6.5\end{array}$ & $\begin{array}{l}184961 \\
.8\end{array}$ & $\begin{array}{l}297303 \\
.8\end{array}$ & $\begin{array}{l}355568 \\
.8\end{array}$ & $\begin{array}{l}372898 \\
.7\end{array}$ & $\begin{array}{l}279917 \\
.3\end{array}$ & 0.0 & 0.0 & 0.0 & 0.0 & 0.0 & 0.0 \\
\hline $\operatorname{PI}(38: 7)$ & 880.5 & 9.4 & $\mathrm{C} 47 \mathrm{H} 77 \mathrm{O} 13 \mathrm{P}$ & $\begin{array}{l}\text { Glyceroph } \\
\text { ospholipid } \\
\text { s }\end{array}$ & $\begin{array}{l}\text { Glyceroph } \\
\text { osphoinosi } \\
\text { tols }\end{array}$ & 0.0 & 0.0 & 2793.1 & 5683.1 & 8744.9 & 0.0 & 0.0 & 0.0 & 0.0 & 0.0 & 0.0 & 0.0 \\
\hline $\operatorname{PI}(39: 4)$ & 900.6 & 11.6 & $\mathrm{C} 48 \mathrm{H} 85 \mathrm{O} 13 \mathrm{P}$ & $\begin{array}{l}\text { Glyceroph } \\
\text { ospholipid } \\
\text { s }\end{array}$ & $\begin{array}{l}\text { Glyceroph } \\
\text { osphoinosi } \\
\text { tols }\end{array}$ & $\begin{array}{l}29675 \\
6.9\end{array}$ & $\begin{array}{l}487008 \\
.9\end{array}$ & $\begin{array}{l}705226 \\
.1\end{array}$ & $\begin{array}{l}675968 \\
.3\end{array}$ & $\begin{array}{l}733749 \\
.4\end{array}$ & $\begin{array}{l}665261 \\
.3\end{array}$ & 0.0 & 0.0 & 0.0 & 0.0 & 0.0 & 0.0 \\
\hline $\operatorname{PI}(39: 5)$ & 898.6 & 11.0 & $\mathrm{C} 48 \mathrm{H} 83 \mathrm{O} 13 \mathrm{P}$ & $\begin{array}{l}\text { Glyceroph } \\
\text { ospholipid } \\
\text { s }\end{array}$ & $\begin{array}{l}\text { Glyceroph } \\
\text { osphoinosi } \\
\text { tols }\end{array}$ & $\begin{array}{l}83346 \\
.8\end{array}$ & $\begin{array}{l}118677 \\
.3\end{array}$ & $\begin{array}{l}204793 \\
.5\end{array}$ & $\begin{array}{l}305574 \\
.8\end{array}$ & $\begin{array}{l}572686 \\
.2\end{array}$ & $\begin{array}{l}193238 \\
.1\end{array}$ & 0.0 & 0.0 & 0.0 & 0.0 & 0.0 & 0.0 \\
\hline $\operatorname{PI}(39: 6)$ & 896.5 & 10.4 & C48H81O13P & $\begin{array}{l}\text { Glyceroph } \\
\text { ospholipid } \\
\mathrm{s}\end{array}$ & $\begin{array}{l}\text { Glyceroph } \\
\text { osphoinosi } \\
\text { tols }\end{array}$ & $\begin{array}{l}9001 . \\
9\end{array}$ & 6890.0 & 8739.7 & 8165.2 & $\begin{array}{l}17729 . \\
6\end{array}$ & 9255.6 & 0.0 & 0.0 & 0.0 & 0.0 & 0.0 & 0.0 \\
\hline $\operatorname{PI}(40: 0)$ & 939.7 & 12.8 & $\mathrm{C} 49 \mathrm{H} 95 \mathrm{O} 13 \mathrm{P}$ & $\begin{array}{l}\text { Glyceroph } \\
\text { ospholipid } \\
\text { s }\end{array}$ & $\begin{array}{l}\text { Glyceroph } \\
\text { osphoinosi } \\
\text { tols }\end{array}$ & $\begin{array}{l}43711 \\
.7\end{array}$ & $\begin{array}{l}54316 . \\
3\end{array}$ & $\begin{array}{l}85101 . \\
9\end{array}$ & $\begin{array}{l}96632 . \\
4\end{array}$ & $\begin{array}{l}101351 \\
.0\end{array}$ & $\begin{array}{l}89495 . \\
1\end{array}$ & 0.0 & 0.0 & 0.0 & 0.0 & 0.0 & 0.0 \\
\hline $\operatorname{PI}(40: 5)$ & 912.6 & 11.3 & C49H85O13P & $\begin{array}{l}\text { Glyceroph } \\
\text { ospholipid } \\
\text { s }\end{array}$ & $\begin{array}{l}\text { Glyceroph } \\
\text { osphoinosi } \\
\text { tols }\end{array}$ & $\begin{array}{l}12448 \\
.0\end{array}$ & $\begin{array}{l}18704 . \\
6\end{array}$ & $\begin{array}{l}22452 . \\
5\end{array}$ & $\begin{array}{l}19372 . \\
8\end{array}$ & $\begin{array}{l}28681 . \\
0\end{array}$ & $\begin{array}{l}26595 . \\
3\end{array}$ & 0.0 & 0.0 & 0.0 & 0.0 & 0.0 & 0.0 \\
\hline $\operatorname{PI}(40: 6)$ & 910.6 & 10.8 & C49H83O13P & $\begin{array}{l}\text { Glyceroph } \\
\text { ospholipid } \\
\text { s }\end{array}$ & $\begin{array}{l}\text { Glyceroph } \\
\text { osphoinosi } \\
\text { tols }\end{array}$ & $\begin{array}{l}18521 \\
.2\end{array}$ & $\begin{array}{l}31216 . \\
1\end{array}$ & $\begin{array}{l}41006 . \\
0\end{array}$ & $\begin{array}{l}43388 . \\
1\end{array}$ & $\begin{array}{l}43157 . \\
5\end{array}$ & $\begin{array}{l}33048 . \\
8\end{array}$ & 0.0 & 0.0 & 0.0 & 0.0 & 0.0 & 0.0 \\
\hline $\operatorname{PI}(40: 7)$ & 908.5 & 10.3 & $\mathrm{C} 49 \mathrm{H} 81 \mathrm{O} 13 \mathrm{P}$ & $\begin{array}{l}\text { Glyceroph } \\
\text { ospholipid } \\
\text { s }\end{array}$ & $\begin{array}{l}\text { Glyceroph } \\
\text { osphoinosi } \\
\text { tols }\end{array}$ & $\begin{array}{l}72730 \\
.7\end{array}$ & $\begin{array}{l}118275 \\
.2\end{array}$ & $\begin{array}{l}180472 \\
.8\end{array}$ & $\begin{array}{l}184146 \\
.4\end{array}$ & $\begin{array}{l}144242 \\
.0\end{array}$ & $\begin{array}{l}189037 \\
.1\end{array}$ & 0.0 & 0.0 & 0.0 & 0.0 & 0.0 & 0.0 \\
\hline $\operatorname{PI}(40: 8)$ & 906.5 & 9.9 & C49H79O13P & $\begin{array}{l}\text { Glyceroph } \\
\text { ospholipid } \\
\text { s }\end{array}$ & $\begin{array}{l}\text { Glyceroph } \\
\text { osphoinosi } \\
\text { tols }\end{array}$ & $\begin{array}{l}79782 \\
.3\end{array}$ & $\begin{array}{l}131292 \\
.7\end{array}$ & $\begin{array}{l}177243 \\
.9\end{array}$ & $\begin{array}{l}178154 \\
.4\end{array}$ & $\begin{array}{l}142643 \\
.5\end{array}$ & $\begin{array}{l}178403 \\
.9\end{array}$ & 0.0 & 0.0 & 0.0 & 0.0 & 0.0 & 0.0 \\
\hline $\mathrm{PI}(\mathrm{O}-33: 1)$ & 808.5 & 12.2 & $\mathrm{C} 42 \mathrm{H} 81 \mathrm{O} 12 \mathrm{P}$ & $\begin{array}{l}\text { Glyceroph } \\
\text { ospholipid } \\
\text { s }\end{array}$ & $\begin{array}{l}\text { Glyceroph } \\
\text { osphoinosi } \\
\text { tols }\end{array}$ & $\begin{array}{l}9423 . \\
0\end{array}$ & 0.0 & $\begin{array}{l}14231 . \\
6\end{array}$ & $\begin{array}{l}16972 . \\
3\end{array}$ & 0.0 & 0.0 & 0.0 & 0.0 & 0.0 & 0.0 & 0.0 & 0.0 \\
\hline
\end{tabular}




\begin{tabular}{|c|c|c|c|c|c|c|c|c|c|c|c|c|c|c|c|c|c|}
\hline $\mathrm{PI}(\mathrm{O}-35: 1)$ & 836.6 & 11.8 & $\mathrm{C} 44 \mathrm{H} 85 \mathrm{O} 12 \mathrm{P}$ & $\begin{array}{l}\text { Glyceroph } \\
\text { ospholipid } \\
\mathrm{s}\end{array}$ & $\begin{array}{l}\text { Glyceroph } \\
\text { osphoinosi } \\
\text { tols }\end{array}$ & $\begin{array}{l}10490 \\
.0\end{array}$ & $\begin{array}{l}13677 . \\
7\end{array}$ & $\begin{array}{l}22649 . \\
2\end{array}$ & $\begin{array}{l}15189 . \\
8\end{array}$ & $\begin{array}{l}13427 . \\
9\end{array}$ & $\begin{array}{l}17870 . \\
6\end{array}$ & 0.0 & 0.0 & 0.0 & 0.0 & 0.0 & 0.0 \\
\hline $\mathrm{PI}(\mathrm{O}-36: 1)$ & 850.6 & 12.4 & $\mathrm{C} 45 \mathrm{H} 87 \mathrm{O} 12 \mathrm{P}$ & $\begin{array}{l}\text { Glyceroph } \\
\text { ospholipid } \\
\mathrm{s}\end{array}$ & $\begin{array}{l}\text { Glyceroph } \\
\text { osphoinosi } \\
\text { tols }\end{array}$ & $\begin{array}{l}21960 \\
.1\end{array}$ & $\begin{array}{l}54706 . \\
4\end{array}$ & $\begin{array}{l}47078 \\
8\end{array}$ & $\begin{array}{l}42165 \\
2\end{array}$ & $\begin{array}{l}43489 . \\
2\end{array}$ & $\begin{array}{l}43123 . \\
4\end{array}$ & 0.0 & 0.0 & 0.0 & 0.0 & 0.0 & 0.0 \\
\hline PIP2(36:4) & 1018.5 & 1.1 & $\begin{array}{l}\mathrm{C} 45 \mathrm{H} 81 \mathrm{O} 19 \mathrm{P} \\
3\end{array}$ & $\begin{array}{l}\text { Glyceroph } \\
\text { ospholipid } \\
\mathrm{s}\end{array}$ & $\begin{array}{l}\text { Glyceroph } \\
\text { osphoinosi } \\
\text { tols }\end{array}$ & $\begin{array}{l}37483 \\
4.9\end{array}$ & $\begin{array}{l}254983 \\
.9\end{array}$ & $\begin{array}{l}122463 \\
.5\end{array}$ & $\begin{array}{l}426521 \\
.6\end{array}$ & 0.0 & $\begin{array}{l}527901 \\
.6\end{array}$ & 0.0 & 0.0 & 0.0 & 0.0 & 0.0 & 0.0 \\
\hline $\operatorname{PS}(15: 0)$ & 637.4 & 6.6 & $\begin{array}{l}\text { C31H60NO10 } \\
P\end{array}$ & $\begin{array}{l}\text { Glyceroph } \\
\text { ospholipid } \\
\mathrm{s}\end{array}$ & $\begin{array}{l}\text { Glyceroph } \\
\text { osphoserin } \\
\text { es }\end{array}$ & $\begin{array}{l}10020 \\
.1\end{array}$ & 5549.0 & 4158.2 & 0.0 & 0.0 & 3278.1 & 0.0 & 0.0 & 0.0 & 0.0 & 0.0 & 0.0 \\
\hline $\operatorname{PS}(19: 0 / 0: 0)$ & 539.3 & 5.6 & C25H50NO9P & $\begin{array}{l}\text { Glyceroph } \\
\text { ospholipid } \\
\mathrm{s}\end{array}$ & $\begin{array}{l}\text { Glyceroph } \\
\text { osphoserin } \\
\text { es }\end{array}$ & $\begin{array}{l}11866 \\
5.1\end{array}$ & $\begin{array}{l}127840 \\
.6\end{array}$ & $\begin{array}{l}240679 \\
.0\end{array}$ & $\begin{array}{l}204876 \\
.8\end{array}$ & $\begin{array}{l}126642 \\
.5\end{array}$ & $\begin{array}{l}88581 . \\
6\end{array}$ & 0.0 & 0.0 & 0.0 & 0.0 & 0.0 & 0.0 \\
\hline $\begin{array}{l}\mathrm{PS}\left(19: 1(9 \mathrm{Z}) / 22: 6\left(4 \mathrm{Z} \_7 Z \_10\right.\right. \\
\mathrm{Z} \_13 \mathrm{~B} \text { 16Z_19Z)) }\end{array}$ & 847.5 & 11.2 & $\begin{array}{l}\text { C47H78NO10 } \\
P\end{array}$ & $\begin{array}{l}\text { Glyceroph } \\
\text { ospholipid } \\
\mathrm{s}\end{array}$ & $\begin{array}{l}\text { Glyceroph } \\
\text { osphoserin } \\
\text { es }\end{array}$ & $\begin{array}{l}14049 \\
.4\end{array}$ & $\begin{array}{l}16489 . \\
1\end{array}$ & $\begin{array}{l}23821 \\
2\end{array}$ & $\begin{array}{l}20125 . \\
5\end{array}$ & 8330.6 & $\begin{array}{l}21982 . \\
4\end{array}$ & 0.0 & 0.0 & 0.0 & 0.0 & 0.0 & 0.0 \\
\hline $\mathrm{PS}(20: 0 / 0: 0)$ & 553.3 & 4.4 & $\mathrm{C} 26 \mathrm{H} 52 \mathrm{NO} 9 \mathrm{P}$ & $\begin{array}{l}\text { Glyceroph } \\
\text { ospholipid } \\
\mathrm{s}\end{array}$ & $\begin{array}{l}\text { Glyceroph } \\
\text { osphoserin } \\
\text { es }\end{array}$ & $\begin{array}{l}35556 \\
.0\end{array}$ & $\begin{array}{l}56862 . \\
4\end{array}$ & $\begin{array}{l}107437 \\
.5\end{array}$ & $\begin{array}{l}99740 . \\
7\end{array}$ & $\begin{array}{l}76522 . \\
7\end{array}$ & $\begin{array}{l}65060 \\
2\end{array}$ & 0.0 & 0.0 & 0.0 & 0.0 & 0.0 & 0.0 \\
\hline $\operatorname{PS}(21: 0 / 0: 0)$ & 567.4 & 5.0 & $\mathrm{C} 27 \mathrm{H} 54 \mathrm{NO} 9 \mathrm{P}$ & $\begin{array}{l}\text { Glyceroph } \\
\text { ospholipid } \\
\text { s }\end{array}$ & $\begin{array}{l}\text { Glyceroph } \\
\text { osphoserin } \\
\text { es }\end{array}$ & $\begin{array}{l}10748 \\
0.6\end{array}$ & $\begin{array}{l}122503 \\
.1\end{array}$ & $\begin{array}{l}177261 \\
.5\end{array}$ & $\begin{array}{l}208585 \\
.3\end{array}$ & $\begin{array}{l}214284 \\
.0\end{array}$ & $\begin{array}{l}86806 . \\
3\end{array}$ & 0.0 & 0.0 & 0.0 & 0.0 & 0.0 & 0.0 \\
\hline $\operatorname{PS}(22: 0 / 0: 0)$ & 581.4 & 5.6 & $\mathrm{C} 28 \mathrm{H} 56 \mathrm{NO} 9 \mathrm{P}$ & $\begin{array}{l}\text { Glyceroph } \\
\text { ospholipid } \\
\text { s }\end{array}$ & $\begin{array}{l}\text { Glyceroph } \\
\text { osphoserin } \\
\text { es }\end{array}$ & $\begin{array}{l}7828 . \\
7\end{array}$ & $\begin{array}{l}13056 . \\
4\end{array}$ & $\begin{array}{l}23874 \\
1\end{array}$ & $\begin{array}{l}44891 . \\
4\end{array}$ & $\begin{array}{l}69589 . \\
9\end{array}$ & $\begin{array}{l}12575 . \\
9\end{array}$ & 0.0 & 0.0 & 0.0 & 0.0 & 0.0 & 0.0 \\
\hline $\operatorname{PS}(26: 0))$ & 651.4 & 7.2 & $\begin{array}{l}\mathrm{C} 32 \mathrm{H} 62 \mathrm{NO} 10 \\
\mathrm{P}\end{array}$ & $\begin{array}{l}\text { Glyceroph } \\
\text { ospholipid } \\
\mathrm{s}\end{array}$ & $\begin{array}{l}\text { Glyceroph } \\
\text { osphoserin } \\
\text { es }\end{array}$ & $\begin{array}{l}8375 . \\
8\end{array}$ & 7486.0 & 0.0 & 0.0 & 0.0 & 0.0 & 0.0 & 0.0 & 0.0 & 0.0 & 0.0 & 0.0 \\
\hline $\operatorname{PS}(34: 0)$ & 809.5 & 8.8 & $\begin{array}{l}\mathrm{C} 40 \mathrm{H} 78 \mathrm{NO} 10 \\
\mathrm{P}\end{array}$ & $\begin{array}{l}\text { Glyceroph } \\
\text { ospholipid } \\
\mathrm{s}\end{array}$ & $\begin{array}{l}\text { Glyceroph } \\
\text { osphoserin } \\
\text { es }\end{array}$ & 0.0 & 0.0 & 3425.2 & 5689.8 & 0.0 & 5724.6 & 0.0 & 0.0 & 0.0 & 0.0 & 0.0 & 0.0 \\
\hline $\operatorname{PS}(34: 1)$ & 761.5 & 10.9 & $\begin{array}{l}\mathrm{C} 40 \mathrm{H} 76 \mathrm{NO} 10 \\
\mathrm{P}\end{array}$ & $\begin{array}{l}\text { Glyceroph } \\
\text { ospholipid } \\
\mathrm{s}\end{array}$ & $\begin{array}{l}\text { Glyceroph } \\
\text { osphoserin } \\
\text { es }\end{array}$ & $\begin{array}{l}15841 \\
.9\end{array}$ & $\begin{array}{l}28462 . \\
6\end{array}$ & $\begin{array}{l}39717 . \\
9\end{array}$ & $\begin{array}{l}46187 . \\
7\end{array}$ & $\begin{array}{l}51573 . \\
1\end{array}$ & $\begin{array}{l}39621 . \\
3\end{array}$ & 0.0 & 0.0 & 0.0 & 0.0 & 0.0 & 0.0 \\
\hline PS(35:2) & 773.5 & 10.8 & $\begin{array}{l}\text { C41H76NO10 } \\
P\end{array}$ & $\begin{array}{l}\text { Glyceroph } \\
\text { ospholipid } \\
\text { s }\end{array}$ & $\begin{array}{l}\text { Glyceroph } \\
\text { osphoserin } \\
\text { es }\end{array}$ & $\begin{array}{l}36110 \\
.1\end{array}$ & $\begin{array}{l}58421 . \\
9\end{array}$ & $\begin{array}{l}71402 . \\
3\end{array}$ & $\begin{array}{l}79205 . \\
5\end{array}$ & $\begin{array}{l}103072 \\
.0\end{array}$ & $\begin{array}{l}76741 . \\
3\end{array}$ & 0.0 & 0.0 & 0.0 & 0.0 & 0.0 & 0.0 \\
\hline
\end{tabular}




\begin{tabular}{|c|c|c|c|c|c|c|c|c|c|c|c|c|c|c|c|c|c|}
\hline $\operatorname{PS}(36: 3)$ & 785.5 & 10.8 & $\begin{array}{l}\mathrm{C} 42 \mathrm{H} 76 \mathrm{NO} 10 \\
\mathrm{P}\end{array}$ & $\begin{array}{l}\text { Glyceroph } \\
\text { ospholipid } \\
\mathrm{s}\end{array}$ & $\begin{array}{l}\text { Glyceroph } \\
\text { osphoserin } \\
\text { es }\end{array}$ & $\begin{array}{l}10440 \\
8.3\end{array}$ & $\begin{array}{l}172358 \\
.1\end{array}$ & $\begin{array}{l}231390 \\
.8\end{array}$ & $\begin{array}{l}267139 \\
.5\end{array}$ & $\begin{array}{l}307913 \\
.7\end{array}$ & $\begin{array}{l}236748 \\
.2\end{array}$ & 0.0 & 0.0 & 0.0 & 0.0 & 0.0 & 0.0 \\
\hline $\operatorname{PS}(36: 4)$ & 783.5 & 10.5 & $\begin{array}{l}\mathrm{C} 42 \mathrm{H} 74 \mathrm{NO} 10 \\
\mathrm{P}\end{array}$ & $\begin{array}{l}\text { Glyceroph } \\
\text { ospholipid } \\
\mathrm{s}\end{array}$ & $\begin{array}{l}\text { Glyceroph } \\
\text { osphoserin } \\
\text { es }\end{array}$ & $\begin{array}{l}15017 \\
.8\end{array}$ & $\begin{array}{l}25032 . \\
7\end{array}$ & $\begin{array}{l}34884 . \\
5\end{array}$ & $\begin{array}{l}48683 . \\
2\end{array}$ & $\begin{array}{l}48964 . \\
5\end{array}$ & $\begin{array}{l}45234 . \\
0\end{array}$ & 0.0 & 0.0 & 0.0 & 0.0 & 0.0 & 0.0 \\
\hline $\operatorname{PS}(37: 0)$ & 805.6 & 13.4 & $\begin{array}{l}\mathrm{C} 43 \mathrm{H} 84 \mathrm{NO} 10 \\
\mathrm{P}\end{array}$ & $\begin{array}{l}\text { Glyceroph } \\
\text { ospholipid } \\
\mathrm{s}\end{array}$ & $\begin{array}{l}\text { Glyceroph } \\
\text { osphoserin } \\
\text { es }\end{array}$ & $\begin{array}{l}37640 \\
3.4\end{array}$ & $\begin{array}{l}488172 \\
.5\end{array}$ & $\begin{array}{l}634022 \\
.6\end{array}$ & $\begin{array}{l}685721 \\
.5\end{array}$ & $\begin{array}{l}651095 \\
.9\end{array}$ & $\begin{array}{l}636922 \\
.7\end{array}$ & $\begin{array}{l}11343 . \\
1\end{array}$ & 6214.4 & 7331.1 & 6970.8 & 7463.6 & 5855.6 \\
\hline $\operatorname{PS}(37: 1)$ & 803.6 & 12.6 & $\begin{array}{l}\mathrm{C} 43 \mathrm{H} 82 \mathrm{NO} 10 \\
\mathrm{P}\end{array}$ & $\begin{array}{l}\text { Glyceroph } \\
\text { ospholipid } \\
\mathrm{s}\end{array}$ & $\begin{array}{l}\text { Glyceroph } \\
\text { osphoserin } \\
\text { es }\end{array}$ & $\begin{array}{l}51822 \\
9.6\end{array}$ & $\begin{array}{l}589999 \\
.8\end{array}$ & $\begin{array}{l}856156 \\
.1\end{array}$ & $\begin{array}{l}831662 \\
.3\end{array}$ & $\begin{array}{l}775629 \\
.1\end{array}$ & $\begin{array}{l}822368 \\
.6\end{array}$ & 6787.8 & 0.0 & 0.0 & 0.0 & 0.0 & 0.0 \\
\hline $\operatorname{PS}(37: 2)$ & 801.6 & 12.0 & $\begin{array}{l}\mathrm{C} 43 \mathrm{H} 80 \mathrm{NO} 10 \\
\mathrm{P}\end{array}$ & $\begin{array}{l}\text { Glyceroph } \\
\text { ospholipid } \\
\mathrm{s}\end{array}$ & $\begin{array}{l}\text { Glyceroph } \\
\text { osphoserin } \\
\text { es }\end{array}$ & $\begin{array}{l}15107 \\
6.3\end{array}$ & $\begin{array}{l}184298 \\
.6\end{array}$ & $\begin{array}{l}251278 \\
.9\end{array}$ & $\begin{array}{l}258468 \\
.4\end{array}$ & $\begin{array}{l}273321 \\
.7\end{array}$ & $\begin{array}{l}267209 \\
.6\end{array}$ & 0.0 & 0.0 & 0.0 & 0.0 & 0.0 & 0.0 \\
\hline $\operatorname{PS}(37: 3)$ & 799.5 & 11.2 & $\begin{array}{l}\mathrm{C} 43 \mathrm{H} 78 \mathrm{NO} 10 \\
\mathrm{P}\end{array}$ & $\begin{array}{l}\text { Glyceroph } \\
\text { ospholipid } \\
\mathrm{s}\end{array}$ & $\begin{array}{l}\text { Glyceroph } \\
\text { osphoserin } \\
\text { es }\end{array}$ & $\begin{array}{l}42340 \\
7.7\end{array}$ & $\begin{array}{l}691670 \\
.7\end{array}$ & $\begin{array}{l}951006 \\
.4\end{array}$ & $\begin{array}{l}917711 \\
.9\end{array}$ & $\begin{array}{l}101398 \\
0.8\end{array}$ & $\begin{array}{l}919464 \\
.1\end{array}$ & 0.0 & 0.0 & 0.0 & 0.0 & 0.0 & 0.0 \\
\hline $\operatorname{PS}(37: 4)$ & 797.5 & 10.9 & $\begin{array}{l}\mathrm{C} 43 \mathrm{H} 76 \mathrm{NO} 10 \\
\mathrm{P}\end{array}$ & $\begin{array}{l}\text { Glyceroph } \\
\text { ospholipid } \\
\mathrm{s}\end{array}$ & $\begin{array}{l}\text { Glyceroph } \\
\text { osphoserin } \\
\text { es }\end{array}$ & $\begin{array}{l}23774 \\
1.8\end{array}$ & $\begin{array}{l}426446 \\
.9\end{array}$ & $\begin{array}{l}534278 \\
.9\end{array}$ & $\begin{array}{l}519311 \\
.9\end{array}$ & $\begin{array}{l}528001 \\
.6\end{array}$ & $\begin{array}{l}564437 \\
.8\end{array}$ & 0.0 & 0.0 & 0.0 & 0.0 & 0.0 & 0.0 \\
\hline $\operatorname{PS}(37: 5)$ & 795.5 & 10.5 & $\begin{array}{l}\mathrm{C} 43 \mathrm{H} 74 \mathrm{NO} 10 \\
\mathrm{P}\end{array}$ & $\begin{array}{l}\text { Glyceroph } \\
\text { ospholipid } \\
\mathrm{s}\end{array}$ & $\begin{array}{l}\text { Glyceroph } \\
\text { osphoserin } \\
\text { es }\end{array}$ & $\begin{array}{l}11130 \\
.0\end{array}$ & $\begin{array}{l}10196 . \\
9\end{array}$ & $\begin{array}{l}12692 . \\
1\end{array}$ & $\begin{array}{l}10753 . \\
0\end{array}$ & $\begin{array}{l}14243 . \\
4\end{array}$ & $\begin{array}{l}14009 . \\
1\end{array}$ & 0.0 & 0.0 & 0.0 & 0.0 & 0.0 & 0.0 \\
\hline $\operatorname{PS}(38: 0)$ & 819.6 & 14.1 & $\begin{array}{l}\mathrm{C} 44 \mathrm{H} 86 \mathrm{NO} 10 \\
\mathrm{P}\end{array}$ & $\begin{array}{l}\text { Glyceroph } \\
\text { ospholipid } \\
\mathrm{s}\end{array}$ & $\begin{array}{l}\text { Glyceroph } \\
\text { osphoserin } \\
\text { es }\end{array}$ & $\begin{array}{l}41087 \\
2.7\end{array}$ & $\begin{array}{l}496908 \\
.6\end{array}$ & $\begin{array}{l}601857 \\
.9\end{array}$ & $\begin{array}{l}559830 \\
.5\end{array}$ & $\begin{array}{l}601987 \\
.2\end{array}$ & $\begin{array}{l}586589 \\
.4\end{array}$ & $\begin{array}{l}10459 . \\
4\end{array}$ & 9233.0 & 8374.3 & $\begin{array}{l}10098 . \\
8\end{array}$ & 5882.4 & 6568.4 \\
\hline PS(38:1) & 817.6 & 13.3 & $\begin{array}{l}\text { C44H84NO10 } \\
\mathrm{P}\end{array}$ & $\begin{array}{l}\text { Glyceroph } \\
\text { ospholipid } \\
\text { s }\end{array}$ & $\begin{array}{l}\text { Glyceroph } \\
\text { osphoserin } \\
\text { es }\end{array}$ & $\begin{array}{l}77347 \\
9.1\end{array}$ & $\begin{array}{l}105150 \\
0.8\end{array}$ & $\begin{array}{l}136904 \\
5.9\end{array}$ & $\begin{array}{l}141400 \\
7.5\end{array}$ & $\begin{array}{l}117854 \\
3.1\end{array}$ & $\begin{array}{l}160695 \\
3.6\end{array}$ & $\begin{array}{l}10038 . \\
7\end{array}$ & 0.0 & 0.0 & 0.0 & 0.0 & 0.0 \\
\hline $\operatorname{PS}(38: 2)$ & 815.6 & 12.4 & $\begin{array}{l}\mathrm{C} 44 \mathrm{H} 82 \mathrm{NO} 10 \\
\mathrm{P}\end{array}$ & $\begin{array}{l}\text { Glyceroph } \\
\text { ospholipid } \\
\mathrm{s}\end{array}$ & $\begin{array}{l}\text { Glyceroph } \\
\text { osphoserin } \\
\text { es }\end{array}$ & $\begin{array}{l}96707 \\
9.1\end{array}$ & $\begin{array}{l}147520 \\
9.1\end{array}$ & $\begin{array}{l}198853 \\
4.1\end{array}$ & $\begin{array}{l}221001 \\
0.0\end{array}$ & $\begin{array}{l}221933 \\
2.3\end{array}$ & $\begin{array}{l}218829 \\
1.5\end{array}$ & 6979.3 & 0.0 & 0.0 & 0.0 & 0.0 & 0.0 \\
\hline $\operatorname{PS}(38: 3)$ & 813.6 & 11.7 & $\begin{array}{l}\mathrm{C} 44 \mathrm{H} 80 \mathrm{NO} 10 \\
\mathrm{P}\end{array}$ & $\begin{array}{l}\text { Glyceroph } \\
\text { ospholipid } \\
\mathrm{s}\end{array}$ & $\begin{array}{l}\text { Glyceroph } \\
\text { osphoserin } \\
\text { es }\end{array}$ & $\begin{array}{l}30347 \\
41.3\end{array}$ & $\begin{array}{l}480949 \\
8.5\end{array}$ & $\begin{array}{l}732599 \\
0.5\end{array}$ & $\begin{array}{l}738260 \\
5.5\end{array}$ & $\begin{array}{l}594416 \\
7.0\end{array}$ & $\begin{array}{l}673000 \\
3.0\end{array}$ & $\begin{array}{l}15030 . \\
8\end{array}$ & 0.0 & 3135.4 & 0.0 & 2733.9 & 0.0 \\
\hline PS(38:4) & 811.5 & 11.4 & $\begin{array}{l}\text { C44H78NO10 } \\
\text { P }\end{array}$ & $\begin{array}{l}\text { Glyceroph } \\
\text { ospholipid } \\
\text { s }\end{array}$ & $\begin{array}{l}\text { Glyceroph } \\
\text { osphoserin } \\
\text { es }\end{array}$ & $\begin{array}{l}25817 \\
58.5\end{array}$ & $\begin{array}{l}437436 \\
8.0\end{array}$ & $\begin{array}{l}626664 \\
8.0\end{array}$ & $\begin{array}{l}620156 \\
5.5\end{array}$ & $\begin{array}{l}479856 \\
1.0\end{array}$ & $\begin{array}{l}592774 \\
4.0\end{array}$ & 9347.4 & 0.0 & 0.0 & 0.0 & 0.0 & 0.0 \\
\hline
\end{tabular}




\begin{tabular}{|c|c|c|c|c|c|c|c|c|c|c|c|c|c|c|c|c|c|}
\hline $\operatorname{PS}(38: 5)$ & 809.5 & 11.0 & $\begin{array}{l}\mathrm{C} 44 \mathrm{H} 76 \mathrm{NO} 10 \\
\mathrm{P}\end{array}$ & $\begin{array}{l}\text { Glyceroph } \\
\text { ospholipid } \\
\text { s }\end{array}$ & $\begin{array}{l}\text { Glyceroph } \\
\text { osphoserin } \\
\text { es }\end{array}$ & $\begin{array}{l}11326 \\
1.9\end{array}$ & $\begin{array}{l}111945 \\
.9\end{array}$ & $\begin{array}{l}152432 \\
.1\end{array}$ & $\begin{array}{l}136203 \\
.5\end{array}$ & $\begin{array}{l}131225 \\
.6\end{array}$ & $\begin{array}{l}149570 \\
.2\end{array}$ & 0.0 & 0.0 & 0.0 & 0.0 & 0.0 & 0.0 \\
\hline $\operatorname{PS}(38: 6)$ & 807.5 & 10.4 & $\begin{array}{l}\text { C44H74NO10 } \\
\text { P }\end{array}$ & $\begin{array}{l}\text { Glyceroph } \\
\text { ospholipid } \\
\text { s }\end{array}$ & $\begin{array}{l}\text { Glyceroph } \\
\text { osphoserin } \\
\text { es }\end{array}$ & $\begin{array}{l}3604 . \\
4\end{array}$ & 3990.3 & 5908.7 & 7886.5 & $\begin{array}{l}10450 . \\
9\end{array}$ & 8022.9 & 0.0 & 0.0 & 0.0 & 0.0 & 0.0 & 0.0 \\
\hline $\operatorname{PS}(39: 0)$ & 833.6 & 15.0 & $\begin{array}{l}\text { C45H88NO10 } \\
\text { P }\end{array}$ & $\begin{array}{l}\text { Glyceroph } \\
\text { ospholipid } \\
\text { s }\end{array}$ & $\begin{array}{l}\text { Glyceroph } \\
\text { osphoserin } \\
\text { es }\end{array}$ & $\begin{array}{l}40848 \\
7.9\end{array}$ & $\begin{array}{l}559853 \\
.7\end{array}$ & $\begin{array}{l}823208 \\
.8\end{array}$ & $\begin{array}{l}827886 \\
.5\end{array}$ & $\begin{array}{l}662426 \\
.6\end{array}$ & $\begin{array}{l}747936 \\
.4\end{array}$ & 9448.2 & 5972.0 & 6288.0 & 5295.6 & 6737.8 & 5322.2 \\
\hline $\operatorname{PS}(39: 2)$ & 829.6 & 13.0 & $\begin{array}{l}\mathrm{C} 45 \mathrm{H} 84 \mathrm{NO} 10 \\
\mathrm{P}\end{array}$ & $\begin{array}{l}\text { Glyceroph } \\
\text { ospholipid } \\
\text { s }\end{array}$ & $\begin{array}{l}\text { Glyceroph } \\
\text { osphoserin } \\
\text { es }\end{array}$ & $\begin{array}{l}67176 \\
8.0\end{array}$ & $\begin{array}{l}916832 \\
.1\end{array}$ & $\begin{array}{l}118747 \\
9.0\end{array}$ & $\begin{array}{l}122079 \\
0.3\end{array}$ & $\begin{array}{l}109172 \\
4.1\end{array}$ & $\begin{array}{l}117725 \\
6.4\end{array}$ & 0.0 & 0.0 & 0.0 & 0.0 & 0.0 & 0.0 \\
\hline $\operatorname{PS}(39: 3)$ & 827.6 & 12.6 & $\begin{array}{l}\text { C45H82NO10 } \\
\text { P }\end{array}$ & $\begin{array}{l}\text { Glyceroph } \\
\text { ospholipid } \\
\text { s }\end{array}$ & $\begin{array}{l}\text { Glyceroph } \\
\text { osphoserin } \\
\text { es }\end{array}$ & $\begin{array}{l}65335 \\
8.4\end{array}$ & $\begin{array}{l}942769 \\
.9\end{array}$ & $\begin{array}{l}125295 \\
6.3\end{array}$ & $\begin{array}{l}127641 \\
6.4\end{array}$ & $\begin{array}{l}932167 \\
.9\end{array}$ & $\begin{array}{l}129260 \\
6.0\end{array}$ & 7068.1 & 0.0 & 0.0 & 0.0 & 0.0 & 0.0 \\
\hline $\operatorname{PS}(39: 4)$ & 825.6 & 12.1 & $\begin{array}{l}\text { C45H80NO10 } \\
\text { P }\end{array}$ & $\begin{array}{l}\text { Glyceroph } \\
\text { ospholipid } \\
\text { s }\end{array}$ & $\begin{array}{l}\text { Glyceroph } \\
\text { osphoserin } \\
\text { es }\end{array}$ & $\begin{array}{l}52011 \\
9.3\end{array}$ & $\begin{array}{l}653685 \\
.9\end{array}$ & $\begin{array}{l}955391 \\
.5\end{array}$ & $\begin{array}{l}860577 \\
.4\end{array}$ & $\begin{array}{l}700813 \\
.1\end{array}$ & $\begin{array}{l}887718 \\
.6\end{array}$ & 0.0 & 0.0 & 0.0 & 0.0 & 0.0 & 0.0 \\
\hline $\operatorname{PS}(39: 5)$ & 823.5 & 11.4 & $\begin{array}{l}\mathrm{C} 45 \mathrm{H} 78 \mathrm{NO} 10 \\
\mathrm{P}\end{array}$ & $\begin{array}{l}\text { Glyceroph } \\
\text { ospholipid } \\
\text { s }\end{array}$ & $\begin{array}{l}\text { Glyceroph } \\
\text { osphoserin } \\
\text { es }\end{array}$ & $\begin{array}{l}36183 \\
.6\end{array}$ & $\begin{array}{l}39813 . \\
3\end{array}$ & $\begin{array}{l}50003 . \\
7\end{array}$ & $\begin{array}{l}57004 . \\
2\end{array}$ & $\begin{array}{l}49577 . \\
6\end{array}$ & $\begin{array}{l}34451 . \\
3\end{array}$ & 0.0 & 0.0 & 0.0 & 0.0 & 0.0 & 0.0 \\
\hline $\operatorname{PS}(40: 1)$ & 845.6 & 14.5 & $\begin{array}{l}\mathrm{C} 46 \mathrm{H} 88 \mathrm{NO} 10 \\
\mathrm{P}\end{array}$ & $\begin{array}{l}\text { Glyceroph } \\
\text { ospholipid } \\
\text { s }\end{array}$ & $\begin{array}{l}\text { Glyceroph } \\
\text { osphoserin } \\
\text { es }\end{array}$ & $\begin{array}{l}17528 \\
5.2\end{array}$ & $\begin{array}{l}210850 \\
.0\end{array}$ & $\begin{array}{l}265035 \\
.0\end{array}$ & $\begin{array}{l}296633 \\
.8\end{array}$ & $\begin{array}{l}378532 \\
.1\end{array}$ & $\begin{array}{l}257665 \\
.6\end{array}$ & $\begin{array}{l}55774 . \\
0\end{array}$ & $\begin{array}{l}52056 . \\
6\end{array}$ & $\begin{array}{l}58632 . \\
6\end{array}$ & $\begin{array}{l}50432 . \\
0\end{array}$ & $\begin{array}{l}51438 . \\
4\end{array}$ & $\begin{array}{l}51474 . \\
1\end{array}$ \\
\hline $\operatorname{PS}(40: 2)$ & 843.6 & 13.7 & $\begin{array}{l}\mathrm{C} 46 \mathrm{H} 86 \mathrm{NO} 10 \\
\mathrm{P}\end{array}$ & $\begin{array}{l}\text { Glyceroph } \\
\text { ospholipid } \\
\text { s }\end{array}$ & $\begin{array}{l}\text { Glyceroph } \\
\text { osphoserin } \\
\text { es }\end{array}$ & $\begin{array}{l}62571 \\
4.7\end{array}$ & $\begin{array}{l}849242 \\
.0\end{array}$ & $\begin{array}{l}103655 \\
3.3\end{array}$ & $\begin{array}{l}955281 \\
.4\end{array}$ & $\begin{array}{l}822650 \\
.9\end{array}$ & $\begin{array}{l}106800 \\
5.6\end{array}$ & 0.0 & 0.0 & 0.0 & 0.0 & 0.0 & 0.0 \\
\hline $\operatorname{PS}(40: 3)$ & 841.6 & 13.3 & $\begin{array}{l}\mathrm{C} 46 \mathrm{H} 84 \mathrm{NO} 10 \\
\mathrm{P}\end{array}$ & $\begin{array}{l}\text { Glyceroph } \\
\text { ospholipid } \\
\text { s }\end{array}$ & $\begin{array}{l}\text { Glyceroph } \\
\text { osphoserin } \\
\text { es }\end{array}$ & $\begin{array}{l}15021 \\
85.6\end{array}$ & $\begin{array}{l}211168 \\
9.8\end{array}$ & $\begin{array}{l}283673 \\
4.8\end{array}$ & $\begin{array}{l}247491 \\
2.5\end{array}$ & $\begin{array}{l}145643 \\
1.8\end{array}$ & $\begin{array}{l}338588 \\
7.8\end{array}$ & $\begin{array}{l}13478 . \\
5\end{array}$ & 0.0 & 0.0 & 0.0 & 0.0 & 0.0 \\
\hline $\operatorname{PS}(40: 4)$ & 839.6 & 12.6 & $\begin{array}{l}\text { C46H82NO10 } \\
\text { P }\end{array}$ & $\begin{array}{l}\text { Glyceroph } \\
\text { ospholipid } \\
\mathrm{s}\end{array}$ & $\begin{array}{l}\text { Glyceroph } \\
\text { osphoserin } \\
\text { es }\end{array}$ & $\begin{array}{l}88939 \\
4.3\end{array}$ & $\begin{array}{l}129603 \\
5.4\end{array}$ & $\begin{array}{l}188447 \\
8.6\end{array}$ & $\begin{array}{l}176415 \\
5.6\end{array}$ & $\begin{array}{l}105438 \\
9.1\end{array}$ & $\begin{array}{l}240461 \\
0.5\end{array}$ & 6353.3 & 0.0 & 0.0 & 0.0 & 0.0 & 0.0 \\
\hline $\mathrm{PS}(40: 5)$ & 837.6 & 11.8 & $\begin{array}{l}\text { C46H80NO10 } \\
\text { P }\end{array}$ & $\begin{array}{l}\text { Glyceroph } \\
\text { ospholipid } \\
\text { s }\end{array}$ & $\begin{array}{l}\text { Glyceroph } \\
\text { osphoserin } \\
\text { es }\end{array}$ & $\begin{array}{l}52940 \\
.3\end{array}$ & $\begin{array}{l}58231 . \\
3\end{array}$ & $\begin{array}{l}84540 . \\
6\end{array}$ & $\begin{array}{l}77449 . \\
1\end{array}$ & $\begin{array}{l}69343 . \\
5\end{array}$ & $\begin{array}{l}84984 . \\
1\end{array}$ & 0.0 & 0.0 & 0.0 & 0.0 & 0.0 & 0.0 \\
\hline $\operatorname{PS}(40: 6)$ & 835.5 & 11.5 & $\begin{array}{l}\text { C46H78NO10 } \\
\text { P }\end{array}$ & $\begin{array}{l}\text { Glyceroph } \\
\text { ospholipid } \\
\text { s }\end{array}$ & $\begin{array}{l}\text { Glyceroph } \\
\text { osphoserin } \\
\text { es }\end{array}$ & $\begin{array}{l}71183 \\
.3\end{array}$ & $\begin{array}{l}110526 \\
.1\end{array}$ & $\begin{array}{l}146242 \\
.8\end{array}$ & $\begin{array}{l}164037 \\
.9\end{array}$ & $\begin{array}{l}132319 \\
.6\end{array}$ & $\begin{array}{l}139006 \\
.3\end{array}$ & 0.0 & 0.0 & 0.0 & 0.0 & 0.0 & 0.0 \\
\hline
\end{tabular}




\begin{tabular}{|c|c|c|c|c|c|c|c|c|c|c|c|c|c|c|c|c|c|}
\hline $\operatorname{PS}(41: 1)$ & 859.6 & 15.4 & $\begin{array}{l}\text { C47H90NO10 } \\
\text { P }\end{array}$ & $\begin{array}{l}\text { Glyceroph } \\
\text { ospholipid } \\
\mathrm{s}\end{array}$ & $\begin{array}{l}\text { Glyceroph } \\
\text { osphoserin } \\
\text { es }\end{array}$ & $\begin{array}{l}76791 \\
.6\end{array}$ & $\begin{array}{l}105234 \\
.3\end{array}$ & $\begin{array}{l}144183 \\
.9\end{array}$ & $\begin{array}{l}148128 \\
.1\end{array}$ & $\begin{array}{l}112751 \\
.1\end{array}$ & $\begin{array}{l}133376 \\
.3\end{array}$ & 0.0 & 0.0 & 0.0 & 0.0 & 0.0 & 0.0 \\
\hline $\operatorname{PS}(41: 2)$ & 857.6 & 14.6 & $\begin{array}{l}\text { C47H88NO10 } \\
\text { P }\end{array}$ & $\begin{array}{l}\text { Glyceroph } \\
\text { ospholipid } \\
\text { s }\end{array}$ & $\begin{array}{l}\text { Glyceroph } \\
\text { osphoserin } \\
\text { es }\end{array}$ & $\begin{array}{l}57521 \\
8.1\end{array}$ & $\begin{array}{l}827348 \\
.4\end{array}$ & $\begin{array}{l}109323 \\
4.4\end{array}$ & $\begin{array}{l}111469 \\
2.1\end{array}$ & $\begin{array}{l}721690 \\
.8\end{array}$ & $\begin{array}{l}108521 \\
8.6\end{array}$ & 7194.2 & 0.0 & 0.0 & 0.0 & 0.0 & 0.0 \\
\hline $\operatorname{PS}(41: 3)$ & 855.6 & 14.1 & $\begin{array}{l}\mathrm{C} 47 \mathrm{H} 86 \mathrm{NO} 10 \\
\mathrm{P}\end{array}$ & $\begin{array}{l}\text { Glyceroph } \\
\text { ospholipid } \\
\text { s }\end{array}$ & $\begin{array}{l}\text { Glyceroph } \\
\text { osphoserin } \\
\text { es }\end{array}$ & $\begin{array}{l}11688 \\
77.1\end{array}$ & $\begin{array}{l}152077 \\
3.1\end{array}$ & $\begin{array}{l}207271 \\
2.6\end{array}$ & $\begin{array}{l}189668 \\
6.3\end{array}$ & $\begin{array}{l}127460 \\
2.5\end{array}$ & $\begin{array}{l}185471 \\
3.8\end{array}$ & 0.0 & 0.0 & 0.0 & 0.0 & 0.0 & 0.0 \\
\hline $\operatorname{PS}(41: 4)$ & 853.6 & 13.1 & $\begin{array}{l}\text { C47H84NO10 } \\
\mathrm{P}\end{array}$ & $\begin{array}{l}\text { Glyceroph } \\
\text { ospholipid } \\
\text { s }\end{array}$ & $\begin{array}{l}\text { Glyceroph } \\
\text { osphoserin } \\
\text { es }\end{array}$ & $\begin{array}{l}15895 \\
16.3\end{array}$ & $\begin{array}{l}217923 \\
5.3\end{array}$ & $\begin{array}{l}296417 \\
2.0\end{array}$ & $\begin{array}{l}301648 \\
2.8\end{array}$ & $\begin{array}{l}255636 \\
2.5\end{array}$ & $\begin{array}{l}296861 \\
7.0\end{array}$ & $\begin{array}{l}13110 . \\
5\end{array}$ & 0.0 & 0.0 & 0.0 & 0.0 & 0.0 \\
\hline $\mathrm{PS}(41: 5)$ & 851.6 & 12.5 & $\begin{array}{l}\text { C47H82NO10 } \\
\text { P }\end{array}$ & $\begin{array}{l}\text { Glyceroph } \\
\text { ospholipid } \\
\text { s }\end{array}$ & $\begin{array}{l}\text { Glyceroph } \\
\text { osphoserin } \\
\text { es }\end{array}$ & $\begin{array}{l}46573 \\
6.7\end{array}$ & $\begin{array}{l}534455 \\
.9\end{array}$ & $\begin{array}{l}777018 \\
.7\end{array}$ & $\begin{array}{l}799053 \\
.4\end{array}$ & $\begin{array}{l}523983 \\
.0\end{array}$ & $\begin{array}{l}847818 \\
.3\end{array}$ & 0.0 & 0.0 & 0.0 & 0.0 & 0.0 & 0.0 \\
\hline $\operatorname{PS}(41: 6)$ & 849.6 & 11.9 & $\begin{array}{l}\text { C47H80NO10 } \\
\text { P }\end{array}$ & $\begin{array}{l}\text { Glyceroph } \\
\text { ospholipid } \\
\text { s }\end{array}$ & $\begin{array}{l}\text { Glyceroph } \\
\text { osphoserin } \\
\text { es }\end{array}$ & $\begin{array}{l}56344 \\
.8\end{array}$ & $\begin{array}{l}57951 . \\
1\end{array}$ & $\begin{array}{l}82119 . \\
5\end{array}$ & $\begin{array}{l}86497 . \\
6\end{array}$ & $\begin{array}{l}73194 . \\
4\end{array}$ & $\begin{array}{l}97533 . \\
6\end{array}$ & 0.0 & 0.0 & 0.0 & 0.0 & 0.0 & 0.0 \\
\hline $\operatorname{PS}(42: 2)$ & 871.6 & 15.2 & $\begin{array}{l}\text { C48H90NO10 } \\
\text { P }\end{array}$ & $\begin{array}{l}\text { Glyceroph } \\
\text { ospholipid } \\
\text { s }\end{array}$ & $\begin{array}{l}\text { Glyceroph } \\
\text { osphoserin } \\
\text { es }\end{array}$ & $\begin{array}{l}41208 \\
.4\end{array}$ & $\begin{array}{l}59691 . \\
7\end{array}$ & $\begin{array}{l}78816 . \\
7\end{array}$ & $\begin{array}{l}88028 . \\
9\end{array}$ & $\begin{array}{l}59357 . \\
5\end{array}$ & $\begin{array}{l}74206 . \\
3\end{array}$ & 0.0 & 0.0 & 0.0 & 0.0 & 0.0 & 0.0 \\
\hline $\operatorname{PS}(42: 3)$ & 869.6 & 14.5 & $\begin{array}{l}\text { C48H88NO10 } \\
\text { P }\end{array}$ & $\begin{array}{l}\text { Glyceroph } \\
\text { ospholipid } \\
\text { s }\end{array}$ & $\begin{array}{l}\text { Glyceroph } \\
\text { osphoserin } \\
\text { es }\end{array}$ & $\begin{array}{l}13676 \\
7.5\end{array}$ & $\begin{array}{l}174457 \\
.8\end{array}$ & $\begin{array}{l}254434 \\
.3\end{array}$ & $\begin{array}{l}307874 \\
.2\end{array}$ & $\begin{array}{l}400228 \\
.3\end{array}$ & $\begin{array}{l}249677 \\
.9\end{array}$ & 0.0 & 0.0 & 0.0 & 0.0 & 0.0 & 0.0 \\
\hline $\operatorname{PS}(42: 4)$ & 867.6 & 13.8 & $\begin{array}{l}\mathrm{C} 48 \mathrm{H} 86 \mathrm{NO} 10 \\
\mathrm{P}\end{array}$ & $\begin{array}{l}\text { Glyceroph } \\
\text { ospholipid } \\
\text { s }\end{array}$ & $\begin{array}{l}\text { Glyceroph } \\
\text { osphoserin } \\
\text { es }\end{array}$ & $\begin{array}{l}11331 \\
3.0\end{array}$ & $\begin{array}{l}220041 \\
.8\end{array}$ & $\begin{array}{l}316834 \\
.8\end{array}$ & $\begin{array}{l}435576 \\
.6\end{array}$ & $\begin{array}{l}482452 \\
.4\end{array}$ & $\begin{array}{l}295890 \\
.1\end{array}$ & 0.0 & 0.0 & 0.0 & 0.0 & 0.0 & 0.0 \\
\hline $\operatorname{PS}(42: 5)$ & 865.6 & 13.0 & $\begin{array}{l}\text { C48H84NO10 } \\
\text { P }\end{array}$ & $\begin{array}{l}\text { Glyceroph } \\
\text { ospholipid } \\
\text { s }\end{array}$ & $\begin{array}{l}\text { Glyceroph } \\
\text { osphoserin } \\
\text { es }\end{array}$ & $\begin{array}{l}29967 \\
.7\end{array}$ & $\begin{array}{l}40399 . \\
5\end{array}$ & $\begin{array}{l}63762 . \\
7\end{array}$ & $\begin{array}{l}66302 . \\
4\end{array}$ & $\begin{array}{l}44935 . \\
5\end{array}$ & $\begin{array}{l}66572 . \\
0\end{array}$ & 0.0 & 0.0 & 0.0 & 0.0 & 0.0 & 0.0 \\
\hline $\mathrm{PS}(42: 6)$ & 863.6 & 12.3 & $\begin{array}{l}\text { C48H82NO10 } \\
\text { P }\end{array}$ & $\begin{array}{l}\text { Glyceroph } \\
\text { ospholipid } \\
\text { s }\end{array}$ & $\begin{array}{l}\text { Glyceroph } \\
\text { osphoserin } \\
\text { es }\end{array}$ & $\begin{array}{l}30184 \\
.0\end{array}$ & $\begin{array}{l}49221 . \\
8\end{array}$ & $\begin{array}{l}75206 . \\
2\end{array}$ & $\begin{array}{l}73839 . \\
1\end{array}$ & $\begin{array}{l}47918 . \\
2\end{array}$ & $\begin{array}{l}64921 . \\
6\end{array}$ & 0.0 & 0.0 & 0.0 & 0.0 & 0.0 & 0.0 \\
\hline $\operatorname{PS}(43: 4)$ & 881.6 & 14.1 & $\begin{array}{l}\text { C49H88NO10 } \\
\text { P }\end{array}$ & $\begin{array}{l}\text { Glyceroph } \\
\text { ospholipid } \\
\text { s }\end{array}$ & $\begin{array}{l}\text { Glyceroph } \\
\text { osphoserin } \\
\text { es }\end{array}$ & $\begin{array}{l}12447 \\
4.7\end{array}$ & $\begin{array}{l}148193 \\
.3\end{array}$ & $\begin{array}{l}211654 \\
.8\end{array}$ & $\begin{array}{l}185170 \\
.6\end{array}$ & $\begin{array}{l}175733 \\
.6\end{array}$ & $\begin{array}{l}217763 \\
.6\end{array}$ & 0.0 & 0.0 & 0.0 & 0.0 & 0.0 & 0.0 \\
\hline $\operatorname{PS}(43: 6)$ & 877.6 & 12.9 & $\begin{array}{l}\text { C49H84NO10 } \\
\text { P }\end{array}$ & $\begin{array}{l}\text { Glyceroph } \\
\text { ospholipid } \\
\text { s }\end{array}$ & $\begin{array}{l}\text { Glyceroph } \\
\text { osphoserin } \\
\text { es }\end{array}$ & $\begin{array}{l}12760 \\
66.9\end{array}$ & $\begin{array}{l}189960 \\
3.3\end{array}$ & $\begin{array}{l}272138 \\
5.8\end{array}$ & $\begin{array}{l}271910 \\
7.0\end{array}$ & $\begin{array}{l}162612 \\
1.5\end{array}$ & $\begin{array}{l}303987 \\
0.3\end{array}$ & 7035.1 & 0.0 & 0.0 & 0.0 & 0.0 & 0.0 \\
\hline
\end{tabular}




\begin{tabular}{|c|c|c|c|c|c|c|c|c|c|c|c|c|c|c|c|c|c|}
\hline $\mathrm{PS}\left(\mathrm{O}-20: 0 / 17: 2\left(9 \mathrm{Z} \_12 \mathrm{Z}\right)\right)$ & 787.6 & 13.2 & $\mathrm{C} 43 \mathrm{H} 82 \mathrm{NO} 9 \mathrm{P}$ & $\begin{array}{l}\text { Glyceroph } \\
\text { ospholipid } \\
\text { s }\end{array}$ & $\begin{array}{l}\text { Glyceroph } \\
\text { osphoserin } \\
\text { es }\end{array}$ & $\begin{array}{l}64734 \\
6.8\end{array}$ & $\begin{array}{l}417004 \\
.9\end{array}$ & $\begin{array}{l}735898 \\
.9\end{array}$ & $\begin{array}{l}593709 \\
.1\end{array}$ & $\begin{array}{l}286945 \\
.5\end{array}$ & $\begin{array}{l}521115 \\
.2\end{array}$ & 0.0 & 0.0 & 0.0 & 0.0 & 0.0 & 0.0 \\
\hline $\mathrm{PS}(\mathrm{O}-20: 0 / 18: 0)$ & 805.6 & 15.4 & C44H88NO9P & $\begin{array}{l}\text { Glyceroph } \\
\text { ospholipid } \\
\text { s }\end{array}$ & $\begin{array}{l}\text { Glyceroph } \\
\text { osphoserin } \\
\text { es }\end{array}$ & $\begin{array}{l}41646 \\
.1\end{array}$ & $\begin{array}{l}62086 . \\
3\end{array}$ & $\begin{array}{l}91601 . \\
9\end{array}$ & $\begin{array}{l}99744 . \\
7\end{array}$ & $\begin{array}{l}67212 . \\
4\end{array}$ & $\begin{array}{l}114864 \\
.9\end{array}$ & 0.0 & 0.0 & 0.0 & 0.0 & 0.0 & 0.0 \\
\hline PS(O-34:0) & 749.6 & 12.5 & $\mathrm{C} 40 \mathrm{H} 80 \mathrm{NO} 9 \mathrm{P}$ & $\begin{array}{l}\text { Glyceroph } \\
\text { ospholipid } \\
\text { s }\end{array}$ & $\begin{array}{l}\text { Glyceroph } \\
\text { osphoserin } \\
\text { es }\end{array}$ & $\begin{array}{l}25796 \\
8.9\end{array}$ & $\begin{array}{l}208950 \\
.6\end{array}$ & $\begin{array}{l}235530 \\
.2\end{array}$ & $\begin{array}{l}243498 \\
.6\end{array}$ & $\begin{array}{l}155252 \\
.3\end{array}$ & $\begin{array}{l}156210 \\
.1\end{array}$ & 0.0 & 0.0 & 0.0 & 0.0 & 0.0 & 0.0 \\
\hline PS(O-34:1) & 747.5 & 12.3 & $\mathrm{C} 40 \mathrm{H} 78 \mathrm{NO} 9 \mathrm{P}$ & $\begin{array}{l}\text { Glyceroph } \\
\text { ospholipid } \\
\text { s }\end{array}$ & $\begin{array}{l}\text { Glyceroph } \\
\text { osphoserin } \\
\text { es }\end{array}$ & $\begin{array}{l}37939 \\
0.2\end{array}$ & $\begin{array}{l}277005 \\
.1\end{array}$ & $\begin{array}{l}413005 \\
.4\end{array}$ & $\begin{array}{l}417301 \\
.8\end{array}$ & $\begin{array}{l}302715 \\
.2\end{array}$ & $\begin{array}{l}315563 \\
.5\end{array}$ & 0.0 & 0.0 & 0.0 & 0.0 & 0.0 & 0.0 \\
\hline $\mathrm{PS}(\mathrm{O}-35: 2)$ & 759.5 & 12.9 & C41H78NO9P & $\begin{array}{l}\text { Glyceroph } \\
\text { ospholipid } \\
\text { s }\end{array}$ & $\begin{array}{l}\text { Glyceroph } \\
\text { osphoserin } \\
\text { es }\end{array}$ & $\begin{array}{l}71937 \\
.3\end{array}$ & $\begin{array}{l}78656 . \\
4\end{array}$ & $\begin{array}{l}68233 . \\
7\end{array}$ & $\begin{array}{l}85273 . \\
0\end{array}$ & $\begin{array}{l}127872 \\
.3\end{array}$ & $\begin{array}{l}53599 . \\
1\end{array}$ & 0.0 & 0.0 & 0.0 & 0.0 & 0.0 & 0.0 \\
\hline $\mathrm{PS}(\mathrm{O}-36: 1)$ & 775.6 & 13.0 & $\mathrm{C} 42 \mathrm{H} 82 \mathrm{NO} 9 \mathrm{P}$ & $\begin{array}{l}\text { Glyceroph } \\
\text { ospholipid } \\
\text { s }\end{array}$ & $\begin{array}{l}\text { Glyceroph } \\
\text { osphoserin } \\
\text { es }\end{array}$ & $\begin{array}{l}28964 \\
9.6\end{array}$ & $\begin{array}{l}206811 \\
.0\end{array}$ & $\begin{array}{l}301813 \\
.6\end{array}$ & $\begin{array}{l}208481 \\
.0\end{array}$ & $\begin{array}{l}79408 . \\
0\end{array}$ & $\begin{array}{l}242364 \\
.1\end{array}$ & 0.0 & 0.0 & 0.0 & 0.0 & 0.0 & 0.0 \\
\hline $\mathrm{PS}(\mathrm{O}-36: 2)$ & 773.6 & 12.9 & $\mathrm{C} 42 \mathrm{H} 80 \mathrm{NO} 9 \mathrm{P}$ & $\begin{array}{l}\text { Glyceroph } \\
\text { ospholipid } \\
\text { s }\end{array}$ & $\begin{array}{l}\text { Glyceroph } \\
\text { osphoserin } \\
\text { es }\end{array}$ & $\begin{array}{l}22039 \\
7.2\end{array}$ & $\begin{array}{l}152348 \\
.0\end{array}$ & $\begin{array}{l}293533 \\
.3\end{array}$ & $\begin{array}{l}208065 \\
.7\end{array}$ & $\begin{array}{l}60152 . \\
5\end{array}$ & $\begin{array}{l}262172 \\
.8\end{array}$ & 0.0 & 0.0 & 0.0 & 0.0 & 0.0 & 0.0 \\
\hline PS(O-37:1) & 789.6 & 13.5 & $\mathrm{C} 43 \mathrm{H} 84 \mathrm{NO} 9 \mathrm{P}$ & $\begin{array}{l}\text { Glyceroph } \\
\text { ospholipid } \\
\text { s }\end{array}$ & $\begin{array}{l}\text { Glyceroph } \\
\text { osphoserin } \\
\text { es }\end{array}$ & $\begin{array}{l}28629 \\
1.4\end{array}$ & $\begin{array}{l}190825 \\
.8\end{array}$ & $\begin{array}{l}266904 \\
.8\end{array}$ & $\begin{array}{l}233275 \\
.0\end{array}$ & $\begin{array}{l}105111 \\
.6\end{array}$ & $\begin{array}{l}205134 \\
.3\end{array}$ & 0.0 & 0.0 & 0.0 & 0.0 & 0.0 & 0.0 \\
\hline $\mathrm{PS}(\mathrm{O}-39: 0)$ & 819.6 & 16.0 & $\mathrm{C} 45 \mathrm{H} 90 \mathrm{NO} 9 \mathrm{P}$ & $\begin{array}{l}\text { Glyceroph } \\
\text { ospholipid } \\
\text { s }\end{array}$ & $\begin{array}{l}\text { Glyceroph } \\
\text { osphoserin } \\
\text { es }\end{array}$ & $\begin{array}{l}20629 \\
9.7\end{array}$ & $\begin{array}{l}320824 \\
.3\end{array}$ & $\begin{array}{l}447279 \\
.8\end{array}$ & $\begin{array}{l}499264 \\
.5\end{array}$ & $\begin{array}{l}369288 \\
.4\end{array}$ & $\begin{array}{l}417730 \\
.6\end{array}$ & 0.0 & 0.0 & 0.0 & 0.0 & 0.0 & 0.0 \\
\hline PS(O-42:6) & 849.6 & 12.5 & C48H84NO9P & $\begin{array}{l}\text { Glyceroph } \\
\text { ospholipid } \\
\text { S }\end{array}$ & $\begin{array}{l}\text { Glyceroph } \\
\text { osphoserin } \\
\text { es }\end{array}$ & $\begin{array}{l}15081 \\
.8\end{array}$ & 0.0 & 0.0 & 0.0 & 0.0 & 0.0 & 0.0 & 0.0 & 0.0 & 0.0 & 0.0 & 0.0 \\
\hline $\begin{array}{l}\text { PS(P- } \\
\text { 18:0/22:6(4Z_7Z_10Z_13Z_1 } \\
\text { 6Z_19Z)) }\end{array}$ & 819.5 & 13.8 & $\mathrm{C} 46 \mathrm{H} 78 \mathrm{NO} 9 \mathrm{P}$ & $\begin{array}{l}\text { Glyceroph } \\
\text { ospholipid } \\
\text { s }\end{array}$ & $\begin{array}{l}\text { Glyceroph } \\
\text { osphoserin } \\
\text { es }\end{array}$ & $\begin{array}{l}77895 \\
.3\end{array}$ & 0.0 & $\begin{array}{l}134837 \\
.5\end{array}$ & $\begin{array}{l}115458 \\
.8\end{array}$ & $\begin{array}{l}90395 . \\
1\end{array}$ & $\begin{array}{l}118679 \\
.1\end{array}$ & 0.0 & 0.0 & 0.0 & 0.0 & 0.0 & 0.0 \\
\hline PS(P-39:0) & 817.6 & 15.2 & C45H88NO9P & $\begin{array}{l}\text { Glyceroph } \\
\text { ospholipid } \\
\mathrm{s}\end{array}$ & $\begin{array}{l}\text { Glyceroph } \\
\text { osphoserin } \\
\text { es }\end{array}$ & $\begin{array}{l}13321 \\
8.7\end{array}$ & $\begin{array}{l}200220 \\
.6\end{array}$ & $\begin{array}{l}262608 \\
.8\end{array}$ & $\begin{array}{l}290592 \\
.2\end{array}$ & $\begin{array}{l}234426 \\
.3\end{array}$ & $\begin{array}{l}264244 \\
.9\end{array}$ & 0.0 & 0.0 & 0.0 & 0.0 & 0.0 & 0.0 \\
\hline
\end{tabular}




\begin{tabular}{|c|c|c|c|c|c|c|c|c|c|c|c|c|c|c|c|c|c|}
\hline PS(P-42:6) & 847.6 & 11.9 & $\mathrm{C} 48 \mathrm{H} 82 \mathrm{NO} 9 \mathrm{P}$ & $\begin{array}{l}\text { Glyceroph } \\
\text { ospholipid } \\
\mathrm{s}\end{array}$ & $\begin{array}{l}\text { Glyceroph } \\
\text { osphoserin } \\
\text { es }\end{array}$ & $\begin{array}{l}13894 \\
.1\end{array}$ & 0.0 & 0.0 & 0.0 & 0.0 & 0.0 & 0.0 & 0.0 & 0.0 & 0.0 & 0.0 & 0.0 \\
\hline $\begin{array}{l}\text { Quercetin 3-glucuronide-7- } \\
\text { sulfate }\end{array}$ & 558.0 & 1.4 & $\mathrm{C} 21 \mathrm{H} 18 \mathrm{O} 16 \mathrm{~S}$ & $\begin{array}{l}\text { Polyketid } \\
\text { es }\end{array}$ & Flavonoids & 0.0 & $\begin{array}{l}18737 . \\
3\end{array}$ & 0.0 & 0.0 & 0.0 & $\begin{array}{l}275357 \\
.0\end{array}$ & 0.0 & 0.0 & 0.0 & 0.0 & 0.0 & 0.0 \\
\hline $\operatorname{SM}(\mathrm{d} 17: 1 / 24: 1(15 Z))$ & 844.7 & 14.6 & $\begin{array}{l}\text { C46H91N2O6 } \\
P\end{array}$ & $\begin{array}{l}\text { Sphingoli } \\
\text { pids }\end{array}$ & $\begin{array}{l}\text { Ceramide } \\
\text { phosphoch } \\
\text { olines } \\
\text { (sphingom } \\
\text { yelins) }\end{array}$ & $\begin{array}{l}77389 \\
.4\end{array}$ & $\begin{array}{l}98807 . \\
9\end{array}$ & $\begin{array}{l}136780 \\
.0\end{array}$ & $\begin{array}{l}106369 \\
.8\end{array}$ & $\begin{array}{l}57149 . \\
5\end{array}$ & $\begin{array}{l}174281 \\
.2\end{array}$ & 0.0 & 0.0 & 0.0 & 0.0 & 0.0 & 0.0 \\
\hline $\operatorname{SM}(\mathrm{d} 18: 0 / 16: 0)$ & 705.6 & 17.8 & $\begin{array}{l}\mathrm{C} 39 \mathrm{H} 82 \mathrm{~N} 2 \mathrm{O} 6 \\
\mathrm{P}\end{array}$ & $\begin{array}{l}\text { Sphingoli } \\
\text { pids }\end{array}$ & $\begin{array}{l}\text { Ceramide } \\
\text { phosphoch } \\
\text { olines } \\
\text { (sphingom } \\
\text { yelins) }\end{array}$ & $\begin{array}{l}9182 . \\
8\end{array}$ & $\begin{array}{l}13944 . \\
9\end{array}$ & 7237.6 & $\begin{array}{l}51886 . \\
7\end{array}$ & $\begin{array}{l}11549 . \\
4\end{array}$ & $\begin{array}{l}16743 . \\
6\end{array}$ & 0.0 & 0.0 & 0.0 & 0.0 & 0.0 & 0.0 \\
\hline $\mathrm{SM}(\mathrm{d} 18: 0 / 22: 1(13 \mathrm{Z})(\mathrm{OH}))$ & 800.6 & 13.0 & $\begin{array}{l}\mathrm{C} 45 \mathrm{H} 89 \mathrm{~N} 2 \mathrm{O} 7 \\
\mathrm{P}\end{array}$ & $\begin{array}{l}\text { Sphingoli } \\
\text { pids }\end{array}$ & na & $\begin{array}{l}94358 \\
.5\end{array}$ & $\begin{array}{l}185737 \\
.1\end{array}$ & $\begin{array}{l}277030 \\
.8\end{array}$ & $\begin{array}{l}280639 \\
.6\end{array}$ & $\begin{array}{l}155326 \\
.3\end{array}$ & $\begin{array}{l}394969 \\
.1\end{array}$ & 0.0 & 0.0 & 0.0 & 0.0 & 0.0 & 0.0 \\
\hline $\begin{array}{l}\mathrm{SM}\left(\mathrm{d} 18: 0 / 22: 2\left(13 Z_{-} 16 \mathrm{Z}\right)(\mathrm{O}\right. \\
\mathrm{H}))\end{array}$ & 844.6 & 10.8 & $\begin{array}{l}\mathrm{C} 45 \mathrm{H} 87 \mathrm{~N} 2 \mathrm{O} 7 \\
\mathrm{P}\end{array}$ & $\begin{array}{l}\text { Sphingoli } \\
\text { pids }\end{array}$ & na & $\begin{array}{l}82698 \\
.8\end{array}$ & $\begin{array}{l}127607 \\
.6\end{array}$ & $\begin{array}{l}138112 \\
.0\end{array}$ & $\begin{array}{l}137927 \\
.1\end{array}$ & $\begin{array}{l}73399 . \\
5\end{array}$ & $\begin{array}{l}277010 \\
.3\end{array}$ & 0.0 & 0.0 & 0.0 & 0.0 & 0.0 & 0.0 \\
\hline $\mathrm{SM}(\mathrm{d} 18: 0 / 24: 1(15 \mathrm{Z})(\mathrm{OH}))$ & 828.7 & 14.3 & $\begin{array}{l}\mathrm{C} 47 \mathrm{H} 93 \mathrm{~N} 2 \mathrm{O} 7 \\
P\end{array}$ & $\begin{array}{l}\text { Sphingoli } \\
\text { pids }\end{array}$ & na & $\begin{array}{l}15009 \\
1.8\end{array}$ & $\begin{array}{l}286386 \\
.1\end{array}$ & $\begin{array}{l}473468 \\
.1\end{array}$ & $\begin{array}{l}467664 \\
.3\end{array}$ & $\begin{array}{l}190596 \\
.0\end{array}$ & $\begin{array}{l}641129 \\
.6\end{array}$ & 0.0 & 0.0 & 0.0 & 0.0 & 0.0 & 0.0 \\
\hline $\mathrm{SM}(\mathrm{d} 18: 0 / 24: 1(15 \mathrm{Z}))$ & 814.7 & 16.0 & $\begin{array}{l}\mathrm{C} 47 \mathrm{H} 95 \mathrm{~N} 2 \mathrm{O} 6 \\
\mathrm{P}\end{array}$ & $\begin{array}{l}\text { Sphingoli } \\
\text { pids }\end{array}$ & $\begin{array}{l}\text { Ceramide } \\
\text { phosphoch } \\
\text { olines } \\
\text { (sphingom } \\
\text { yelins) }\end{array}$ & $\begin{array}{l}79082 \\
.6\end{array}$ & $\begin{array}{l}108424 \\
.6\end{array}$ & $\begin{array}{l}179909 \\
.4\end{array}$ & $\begin{array}{l}214100 \\
.1\end{array}$ & $\begin{array}{l}200695 \\
.8\end{array}$ & $\begin{array}{l}179004 \\
.2\end{array}$ & 0.0 & 0.0 & 0.0 & 0.0 & 0.0 & 0.0 \\
\hline $\operatorname{SM}(\mathrm{d} 18: 1 / 24: 1(15 Z))$ & 812.7 & 15.2 & $\begin{array}{l}\mathrm{C} 47 \mathrm{H} 93 \mathrm{~N} 2 \mathrm{O} 6 \\
\mathrm{P}\end{array}$ & $\begin{array}{l}\text { Sphingoli } \\
\text { pids }\end{array}$ & $\begin{array}{l}\text { Ceramide } \\
\text { phosphoch } \\
\text { olines } \\
\text { (sphingom } \\
\text { yelins) }\end{array}$ & $\begin{array}{l}60076 \\
.0\end{array}$ & $\begin{array}{l}95046 . \\
4\end{array}$ & $\begin{array}{l}154329 \\
.9\end{array}$ & $\begin{array}{l}174826 \\
.2\end{array}$ & $\begin{array}{l}45940 . \\
5\end{array}$ & $\begin{array}{l}210681 \\
.4\end{array}$ & 0.0 & 0.0 & 0.0 & 0.0 & 0.0 & 0.0 \\
\hline $\mathrm{SM}(\mathrm{d} 32: 2)$ & 708.5 & 10.2 & $\begin{array}{l}\mathrm{C} 37 \mathrm{H} 73 \mathrm{~N} 2 \mathrm{O} 6 \\
\mathrm{P}\end{array}$ & $\begin{array}{l}\text { Sphingoli } \\
\text { pids }\end{array}$ & $\begin{array}{l}\text { Phosphosp } \\
\text { hingolipids }\end{array}$ & 0.0 & 0.0 & 0.0 & 0.0 & 0.0 & 0.0 & 7271.3 & 0.0 & 3390.0 & 9130.2 & $\begin{array}{l}15322 . \\
2\end{array}$ & 8714.9 \\
\hline $\mathrm{SM}(\mathrm{d} 34: 1)$ & 702.6 & 10.8 & $\begin{array}{l}\text { C39H79N2O6 } \\
P\end{array}$ & $\begin{array}{l}\text { Sphingoli } \\
\text { pids }\end{array}$ & $\begin{array}{l}\text { Phosphosp } \\
\text { hingolipids }\end{array}$ & $\begin{array}{l}13928 \\
.1\end{array}$ & $\begin{array}{l}11539 . \\
3\end{array}$ & $\begin{array}{l}31147 . \\
0\end{array}$ & $\begin{array}{l}26978 . \\
9\end{array}$ & $\begin{array}{l}32463 . \\
3\end{array}$ & $\begin{array}{l}23832 . \\
5\end{array}$ & 0.0 & 0.0 & 0.0 & 0.0 & 0.0 & 0.0 \\
\hline
\end{tabular}




\begin{tabular}{|c|c|c|c|c|c|c|c|c|c|c|c|c|c|c|c|c|c|}
\hline $\mathrm{SM}(\mathrm{d} 35: 1)$ & 716.6 & 11.3 & $\begin{array}{l}\mathrm{C} 40 \mathrm{H} 81 \mathrm{~N} 2 \mathrm{O} 6 \\
\mathrm{P}\end{array}$ & $\begin{array}{l}\text { Sphingoli } \\
\text { pids }\end{array}$ & $\begin{array}{l}\text { Phosphosp } \\
\text { hingolipids }\end{array}$ & $\begin{array}{l}65859 \\
.5\end{array}$ & $\begin{array}{l}111280 \\
.3\end{array}$ & $\begin{array}{l}193097 \\
.1\end{array}$ & $\begin{array}{l}221236 \\
.8\end{array}$ & $\begin{array}{l}276918 \\
.5\end{array}$ & $\begin{array}{l}177946 \\
.0\end{array}$ & 0.0 & 0.0 & 0.0 & 0.0 & 0.0 & 0.0 \\
\hline $\operatorname{SM}(\mathrm{d} 36: 1)$ & 730.6 & 13.3 & $\begin{array}{l}\mathrm{C} 41 \mathrm{H} 83 \mathrm{~N} 2 \mathrm{O} 6 \\
\mathrm{P}\end{array}$ & $\begin{array}{l}\text { Sphingoli } \\
\text { pids }\end{array}$ & $\begin{array}{l}\text { Ceramide } \\
\text { phosphoch } \\
\text { olines } \\
\text { (sphingom } \\
\text { yelins) }\end{array}$ & $\begin{array}{l}10943 \\
0.6\end{array}$ & $\begin{array}{l}214467 \\
.7\end{array}$ & $\begin{array}{l}318450 \\
.3\end{array}$ & $\begin{array}{l}358847 \\
.3\end{array}$ & $\begin{array}{l}384270 \\
.1\end{array}$ & $\begin{array}{l}352701 \\
.7\end{array}$ & 0.0 & 0.0 & 0.0 & 0.0 & 0.0 & 0.0 \\
\hline $\operatorname{SM}(\mathrm{d} 38: 1)$ & 758.6 & 13.2 & $\begin{array}{l}\mathrm{C} 43 \mathrm{H} 87 \mathrm{~N} 2 \mathrm{O} 6 \\
\mathrm{P}\end{array}$ & $\begin{array}{l}\text { Sphingoli } \\
\text { pids }\end{array}$ & $\begin{array}{l}\text { Ceramide } \\
\text { phosphoch } \\
\text { olines } \\
\text { (sphingom } \\
\text { yelins) }\end{array}$ & $\begin{array}{l}58248 \\
3.4\end{array}$ & $\begin{array}{l}956249 \\
.8\end{array}$ & $\begin{array}{l}154587 \\
2.0\end{array}$ & $\begin{array}{l}163063 \\
1.5\end{array}$ & $\begin{array}{l}154173 \\
2.4\end{array}$ & $\begin{array}{l}159448 \\
4.8\end{array}$ & 7296.0 & 0.0 & 0.0 & 0.0 & 0.0 & 0.0 \\
\hline $\mathrm{SM}(\mathrm{d} 39: 1)$ & 772.6 & 13.8 & $\begin{array}{l}\mathrm{C} 44 \mathrm{H} 89 \mathrm{~N} 2 \mathrm{O} 6 \\
\mathrm{P}\end{array}$ & $\begin{array}{l}\text { Sphingoli } \\
\text { pids }\end{array}$ & $\begin{array}{l}\text { Phosphosp } \\
\text { hingolipids }\end{array}$ & $\begin{array}{l}18019 \\
74.5\end{array}$ & $\begin{array}{l}312681 \\
0.3\end{array}$ & $\begin{array}{l}512385 \\
7.5\end{array}$ & $\begin{array}{l}564666 \\
7.0\end{array}$ & $\begin{array}{l}509666 \\
2.5\end{array}$ & $\begin{array}{l}547628 \\
6.5\end{array}$ & $\begin{array}{l}18385 . \\
9\end{array}$ & 0.0 & 4114.3 & 0.0 & 0.0 & 0.0 \\
\hline $\operatorname{SM}(d 40: 1)$ & 786.7 & 14.5 & $\begin{array}{l}\mathrm{C} 45 \mathrm{H} 91 \mathrm{~N} 2 \mathrm{O} 6 \\
\mathrm{P}\end{array}$ & $\begin{array}{l}\text { Sphingoli } \\
\text { pids }\end{array}$ & $\begin{array}{l}\text { Ceramide } \\
\text { phosphoch } \\
\text { olines } \\
\text { (sphingom } \\
\text { yelins) }\end{array}$ & $\begin{array}{l}48090 \\
7.6\end{array}$ & $\begin{array}{l}976707 \\
.2\end{array}$ & $\begin{array}{l}142524 \\
0.1\end{array}$ & $\begin{array}{l}172544 \\
4.3\end{array}$ & $\begin{array}{l}143399 \\
7.6\end{array}$ & $\begin{array}{l}147954 \\
0.0\end{array}$ & 4891.0 & 0.0 & 0.0 & 0.0 & 0.0 & 0.0 \\
\hline $\operatorname{SM}(\mathrm{d} 41: 1)$ & 800.7 & 15.2 & $\begin{array}{l}\mathrm{C} 46 \mathrm{H} 93 \mathrm{~N} 2 \mathrm{O} 6 \\
\mathrm{P}\end{array}$ & $\begin{array}{l}\text { Sphingoli } \\
\text { pids }\end{array}$ & $\begin{array}{l}\text { Phosphosp } \\
\text { hingolipids }\end{array}$ & $\begin{array}{l}12194 \\
0.4\end{array}$ & $\begin{array}{l}155019 \\
.7\end{array}$ & $\begin{array}{l}209624 \\
.3\end{array}$ & $\begin{array}{l}244196 \\
.8\end{array}$ & $\begin{array}{l}263132 \\
.9\end{array}$ & $\begin{array}{l}213447 \\
.0\end{array}$ & 0.0 & 0.0 & 0.0 & 0.0 & 0.0 & 0.0 \\
\hline $\operatorname{SM}(d 43: 1)$ & 828.7 & 16.7 & $\begin{array}{l}\mathrm{C} 48 \mathrm{H} 97 \mathrm{~N} 2 \mathrm{O} 6 \\
\mathrm{P}\end{array}$ & $\begin{array}{l}\text { Sphingoli } \\
\text { pids }\end{array}$ & $\begin{array}{l}\text { Phosphosp } \\
\text { hingolipids }\end{array}$ & $\begin{array}{l}61794 \\
.1\end{array}$ & $\begin{array}{l}81247 . \\
0\end{array}$ & $\begin{array}{l}120374 \\
.2\end{array}$ & $\begin{array}{l}151329 \\
.9\end{array}$ & $\begin{array}{l}138728 \\
.0\end{array}$ & $\begin{array}{l}114460 \\
.7\end{array}$ & 0.0 & 0.0 & 0.0 & 0.0 & 0.0 & 0.0 \\
\hline $\mathrm{SM}(\mathrm{d} 44: 1)$ & 842.7 & 17.5 & $\begin{array}{l}\mathrm{C} 49 \mathrm{H} 99 \mathrm{~N} 2 \mathrm{O} 6 \\
\mathrm{P}\end{array}$ & $\begin{array}{l}\text { Sphingoli } \\
\text { pids }\end{array}$ & $\begin{array}{l}\text { Ceramide } \\
\text { phosphoch } \\
\text { olines } \\
\text { (sphingom } \\
\text { yelins) }\end{array}$ & $\begin{array}{l}9940 . \\
3\end{array}$ & $\begin{array}{l}12663 . \\
6\end{array}$ & $\begin{array}{l}16955 . \\
3\end{array}$ & $\begin{array}{l}17313 . \\
3\end{array}$ & $\begin{array}{l}20122 . \\
6\end{array}$ & $\begin{array}{l}16486 . \\
5\end{array}$ & 0.0 & 0.0 & 0.0 & 0.0 & 0.0 & 0.0 \\
\hline $\begin{array}{l}\text { sn-glycero-3- } \\
\text { Phosphoethanolamine }\end{array}$ & 169.1 & 1.5 & $\mathrm{C} 4 \mathrm{H} 12 \mathrm{NO} 4 \mathrm{P}$ & $\begin{array}{l}\text { Lipid } \\
\text { Metabolis } \\
\mathrm{m}\end{array}$ & $\begin{array}{l}\text { Glyceroph } \\
\text { ospholipid } \\
\text { metabolis } \\
\mathrm{m}\end{array}$ & 0.0 & 0.0 & 0.0 & 0.0 & 0.0 & 0.0 & $\begin{array}{l}142962 \\
7.4\end{array}$ & $\begin{array}{l}120950 \\
4.5\end{array}$ & $\begin{array}{l}141314 \\
0.0\end{array}$ & $\begin{array}{l}152670 \\
8.4\end{array}$ & $\begin{array}{l}133747 \\
6.3\end{array}$ & $\begin{array}{l}169702 \\
1.3\end{array}$ \\
\hline Sphinganine & 301.3 & 8.7 & $\mathrm{C} 18 \mathrm{H} 39 \mathrm{NO} 2$ & $\begin{array}{l}\text { Sphingoli } \\
\text { pids }\end{array}$ & na & $\begin{array}{l}20371 \\
.5\end{array}$ & $\begin{array}{l}14334 . \\
9\end{array}$ & $\begin{array}{l}52914 . \\
6\end{array}$ & $\begin{array}{l}77216 . \\
0\end{array}$ & $\begin{array}{l}88442 . \\
7\end{array}$ & $\begin{array}{l}65641 . \\
7\end{array}$ & $\begin{array}{l}60618 . \\
0\end{array}$ & $\begin{array}{l}62439 . \\
4\end{array}$ & $\begin{array}{l}43992 . \\
9\end{array}$ & $\begin{array}{l}54713 . \\
4\end{array}$ & $\begin{array}{l}75295 . \\
8\end{array}$ & $\begin{array}{l}41796 . \\
1\end{array}$ \\
\hline Stoloniferone F & 479.4 & 8.3 & $\mathrm{C} 28 \mathrm{H} 46 \mathrm{O} 5$ & $\begin{array}{l}\text { Sterol } \\
\text { Lipids }\end{array}$ & Sterols & $\begin{array}{l}3129 . \\
3\end{array}$ & 2564.6 & 0.0 & 3342.3 & 2847.8 & 0.0 & 3243.3 & 3074.8 & 3602.2 & 0.0 & 0.0 & 0.0 \\
\hline Stoloniferone G & 477.3 & 7.9 & $\mathrm{C} 28 \mathrm{H} 44 \mathrm{O} 5$ & $\begin{array}{l}\text { Sterol } \\
\text { Lipids }\end{array}$ & Sterols & 0.0 & 0.0 & 0.0 & 0.0 & 0.0 & 0.0 & 0.0 & 0.0 & 3653.8 & 0.0 & 0.0 & 0.0 \\
\hline
\end{tabular}




\begin{tabular}{|c|c|c|c|c|c|c|c|c|c|c|c|c|c|c|c|c|c|}
\hline Tetracosanoic acid & 368.4 & 12.8 & $\mathrm{C} 24 \mathrm{H} 48 \mathrm{O} 2$ & $\begin{array}{l}\text { Lipid } \\
\text { Metabolis } \\
\mathrm{m}\end{array}$ & $\begin{array}{l}\text { Biosynthes } \\
\text { is of } \\
\text { unsaturate } \\
\text { d fatty } \\
\text { acids }\end{array}$ & $\begin{array}{l}28098 \\
2.0\end{array}$ & $\begin{array}{l}286063 \\
.7\end{array}$ & $\begin{array}{l}450700 \\
.1\end{array}$ & $\begin{array}{l}590596 \\
.6\end{array}$ & $\begin{array}{l}541825 \\
.7\end{array}$ & $\begin{array}{l}477277 \\
.7\end{array}$ & 0.0 & 0.0 & 0.0 & 0.0 & 0.0 & 0.0 \\
\hline Tetradecanoic acid & 228.2 & 5.9 & $\mathrm{C} 14 \mathrm{H} 28 \mathrm{O} 2$ & $\begin{array}{l}\text { Lipid } \\
\text { Metabolis } \\
\mathrm{m}\end{array}$ & $\begin{array}{l}\text { Fatty acid } \\
\text { biosynthes } \\
\text { is }\end{array}$ & $\begin{array}{l}39021 \\
.1\end{array}$ & $\begin{array}{l}35631 . \\
2\end{array}$ & $\begin{array}{l}54867 . \\
8\end{array}$ & $\begin{array}{l}87481 . \\
4\end{array}$ & $\begin{array}{l}100352 \\
.9\end{array}$ & $\begin{array}{l}77931 . \\
8\end{array}$ & 0.0 & 0.0 & 0.0 & 0.0 & 0.0 & 0.0 \\
\hline $\mathrm{TG}(10: 0 / 10: 0 / 10: 0)[$ iso] & 571.5 & 13.9 & С33H62O6 & $\begin{array}{l}\text { Glyceroli } \\
\text { pids }\end{array}$ & $\begin{array}{l}\text { Triacylgly } \\
\text { cerols }\end{array}$ & $\begin{array}{l}39012 \\
.7\end{array}$ & $\begin{array}{l}45381 . \\
5\end{array}$ & $\begin{array}{l}48169 . \\
4\end{array}$ & $\begin{array}{l}74784 . \\
5\end{array}$ & $\begin{array}{l}71272 . \\
2\end{array}$ & $\begin{array}{l}41857 . \\
9\end{array}$ & 0.0 & 0.0 & 0.0 & 0.0 & 0.0 & 0.0 \\
\hline $\mathrm{TG}(38: 1)$ & 681.6 & 18.3 & $\mathrm{C} 41 \mathrm{H} 76 \mathrm{O} 6$ & $\begin{array}{l}\text { Glyceroli } \\
\text { pids }\end{array}$ & $\begin{array}{l}\text { Triacylgly } \\
\text { cerols }\end{array}$ & $\begin{array}{l}30998 \\
.7\end{array}$ & $\begin{array}{l}34957 . \\
9\end{array}$ & $\begin{array}{l}22434 . \\
5\end{array}$ & $\begin{array}{l}113789 \\
.7\end{array}$ & $\begin{array}{l}50920 . \\
5\end{array}$ & $\begin{array}{l}38749 . \\
4\end{array}$ & 0.0 & 0.0 & 0.0 & 0.0 & 0.0 & 0.0 \\
\hline $\mathrm{TG}(40: 0)$ & 711.6 & 20.0 & $\mathrm{C} 43 \mathrm{H} 82 \mathrm{O} 6$ & $\begin{array}{l}\text { Glyceroli } \\
\text { pids }\end{array}$ & $\begin{array}{l}\text { Triacylgly } \\
\text { cerols }\end{array}$ & $\begin{array}{l}75951 \\
.9\end{array}$ & $\begin{array}{l}79690 . \\
4\end{array}$ & $\begin{array}{l}38157 . \\
7\end{array}$ & $\begin{array}{l}158753 \\
.5\end{array}$ & $\begin{array}{l}95680 . \\
9\end{array}$ & $\begin{array}{l}60736 . \\
7\end{array}$ & 0.0 & 0.0 & 0.0 & 0.0 & 0.0 & 0.0 \\
\hline TG(40:2) & 707.6 & 18.8 & $\mathrm{C} 43 \mathrm{H} 78 \mathrm{O} 6$ & $\begin{array}{l}\text { Glyceroli } \\
\text { pids }\end{array}$ & $\begin{array}{l}\text { Triacylgly } \\
\text { cerols }\end{array}$ & $\begin{array}{l}27242 \\
.8\end{array}$ & $\begin{array}{l}40047 . \\
5\end{array}$ & $\begin{array}{l}31403 . \\
5\end{array}$ & $\begin{array}{l}157142 \\
.9\end{array}$ & $\begin{array}{l}53388 . \\
2\end{array}$ & $\begin{array}{l}52948 . \\
4\end{array}$ & 0.0 & 0.0 & 0.0 & 0.0 & 0.0 & 0.0 \\
\hline $\mathrm{TG}(41: 1)$ & 723.6 & 19.9 & $\mathrm{C} 44 \mathrm{H} 82 \mathrm{O} 6$ & $\begin{array}{l}\text { Glyceroli } \\
\text { pids }\end{array}$ & $\begin{array}{l}\text { Triacylgly } \\
\text { cerols }\end{array}$ & $\begin{array}{l}10509 \\
4.4\end{array}$ & $\begin{array}{l}142327 \\
.7\end{array}$ & $\begin{array}{l}80663 . \\
9\end{array}$ & $\begin{array}{l}400465 \\
.8\end{array}$ & $\begin{array}{l}170824 \\
.8\end{array}$ & $\begin{array}{l}177589 \\
.4\end{array}$ & 0.0 & 0.0 & 0.0 & 0.0 & 0.0 & 0.0 \\
\hline $\mathrm{TG}(42: 0)$ & 739.7 & 20.5 & $\mathrm{C} 45 \mathrm{H} 86 \mathrm{O} 6$ & $\begin{array}{l}\text { Glyceroli } \\
\text { pids }\end{array}$ & $\begin{array}{l}\text { Triacylgly } \\
\text { cerols }\end{array}$ & $\begin{array}{l}12880 \\
2.1\end{array}$ & $\begin{array}{l}184924 \\
.6\end{array}$ & $\begin{array}{l}64913 . \\
0\end{array}$ & $\begin{array}{l}264830 \\
.0\end{array}$ & $\begin{array}{l}142089 \\
.2\end{array}$ & $\begin{array}{l}124025 \\
.2\end{array}$ & 0.0 & 0.0 & 0.0 & 0.0 & 0.0 & 0.0 \\
\hline TG(42:1) & 737.7 & 20.2 & $\mathrm{C} 45 \mathrm{H} 84 \mathrm{O} 6$ & $\begin{array}{l}\text { Glyceroli } \\
\text { pids }\end{array}$ & $\begin{array}{l}\text { Triacylgly } \\
\text { cerols }\end{array}$ & $\begin{array}{l}13019 \\
9.3\end{array}$ & $\begin{array}{l}189099 \\
.6\end{array}$ & $\begin{array}{l}90606 . \\
9\end{array}$ & $\begin{array}{l}424927 \\
.9\end{array}$ & $\begin{array}{l}168291 \\
.4\end{array}$ & $\begin{array}{l}204039 \\
.1\end{array}$ & 0.0 & 0.0 & 0.0 & 0.0 & 0.0 & 0.0 \\
\hline $\mathrm{TG}(42: 4)$ & 714.6 & 19.4 & $\mathrm{C} 45 \mathrm{H} 78 \mathrm{O} 6$ & $\begin{array}{l}\text { Glyceroli } \\
\text { pids }\end{array}$ & $\begin{array}{l}\text { Triacylgly } \\
\text { cerols }\end{array}$ & $\begin{array}{l}18104 \\
.0\end{array}$ & $\begin{array}{l}23641 . \\
9\end{array}$ & $\begin{array}{l}14113 . \\
9\end{array}$ & $\begin{array}{l}66901 . \\
1\end{array}$ & $\begin{array}{l}33629 . \\
6\end{array}$ & $\begin{array}{l}27144 . \\
2\end{array}$ & 0.0 & 0.0 & 0.0 & 0.0 & 0.0 & 0.0 \\
\hline $\mathrm{TG}(43: 0)$ & 753.7 & 20.7 & $\mathrm{C} 46 \mathrm{H} 88 \mathrm{O} 6$ & $\begin{array}{l}\text { Glyceroli } \\
\text { pids }\end{array}$ & $\begin{array}{l}\text { Triacylgly } \\
\text { cerols }\end{array}$ & $\begin{array}{l}18210 \\
7.5\end{array}$ & $\begin{array}{l}227286 \\
.6\end{array}$ & $\begin{array}{l}80083 . \\
2\end{array}$ & $\begin{array}{l}292695 \\
.7\end{array}$ & $\begin{array}{l}182878 \\
.4\end{array}$ & $\begin{array}{l}203378 \\
.1\end{array}$ & 0.0 & 0.0 & 0.0 & 0.0 & 0.0 & 0.0 \\
\hline $\mathrm{TG}(43: 3)$ & 730.6 & 20.2 & $\mathrm{C} 46 \mathrm{H} 82 \mathrm{O} 6$ & $\begin{array}{l}\text { Glyceroli } \\
\text { pids }\end{array}$ & $\begin{array}{l}\text { Triacylgly } \\
\text { cerols }\end{array}$ & $\begin{array}{l}16375 \\
.6\end{array}$ & $\begin{array}{l}24704 . \\
5\end{array}$ & $\begin{array}{l}12416 . \\
2\end{array}$ & $\begin{array}{l}44847 . \\
4\end{array}$ & $\begin{array}{l}29460 . \\
4\end{array}$ & $\begin{array}{l}21746 . \\
2\end{array}$ & 0.0 & 0.0 & 0.0 & 0.0 & 0.0 & 0.0 \\
\hline TG(43:4) & 728.6 & 19.8 & $\mathrm{C} 46 \mathrm{H} 80 \mathrm{O} 6$ & $\begin{array}{l}\text { Glyceroli } \\
\text { pids }\end{array}$ & $\begin{array}{l}\text { Triacylgly } \\
\text { cerols }\end{array}$ & $\begin{array}{l}27853 \\
.8\end{array}$ & $\begin{array}{l}39177 . \\
9\end{array}$ & $\begin{array}{l}22611 . \\
8\end{array}$ & $\begin{array}{l}103987 \\
.2\end{array}$ & $\begin{array}{l}42431 . \\
3\end{array}$ & $\begin{array}{l}48581 . \\
6\end{array}$ & 0.0 & 0.0 & 0.0 & 0.0 & 0.0 & 0.0 \\
\hline $\mathrm{TG}(44: 1)$ & 765.7 & 20.7 & $\mathrm{C} 47 \mathrm{H} 88 \mathrm{O} 6$ & $\begin{array}{l}\text { Glyceroli } \\
\text { pids }\end{array}$ & $\begin{array}{l}\text { Triacylgly } \\
\text { cerols }\end{array}$ & $\begin{array}{l}21264 \\
0.0\end{array}$ & $\begin{array}{l}270938 \\
.6\end{array}$ & $\begin{array}{l}124723 \\
.0\end{array}$ & $\begin{array}{l}399087 \\
.3\end{array}$ & $\begin{array}{l}224968 \\
.0\end{array}$ & $\begin{array}{l}278377 \\
.0\end{array}$ & 0.0 & 0.0 & 0.0 & 0.0 & 0.0 & 0.0 \\
\hline $\mathrm{TG}(44: 3)$ & 744.6 & 20.6 & $\mathrm{C} 47 \mathrm{H} 84 \mathrm{O} 6$ & $\begin{array}{l}\text { Glyceroli } \\
\text { pids }\end{array}$ & $\begin{array}{l}\text { Triacylgly } \\
\text { cerols }\end{array}$ & $\begin{array}{l}27351 \\
.6\end{array}$ & $\begin{array}{l}32128 \\
5\end{array}$ & $\begin{array}{l}14517 . \\
0\end{array}$ & $\begin{array}{l}42102 . \\
0\end{array}$ & $\begin{array}{l}34141 . \\
2\end{array}$ & $\begin{array}{l}25211 . \\
9\end{array}$ & 0.0 & 0.0 & 0.0 & 0.0 & 0.0 & 0.0 \\
\hline TG(44:4) & 759.6 & 19.7 & $\mathrm{C} 47 \mathrm{H} 82 \mathrm{O} 6$ & $\begin{array}{l}\text { Glyceroli } \\
\text { pids }\end{array}$ & $\begin{array}{l}\text { Triacylgly } \\
\text { cerols }\end{array}$ & $\begin{array}{l}12613 \\
.2\end{array}$ & $\begin{array}{l}17615 . \\
1\end{array}$ & $\begin{array}{l}13664 . \\
5\end{array}$ & $\begin{array}{l}48198 . \\
9\end{array}$ & $\begin{array}{l}13573 . \\
0\end{array}$ & $\begin{array}{l}21673 . \\
6\end{array}$ & 0.0 & 0.0 & 0.0 & 0.0 & 0.0 & 0.0 \\
\hline
\end{tabular}




\begin{tabular}{|c|c|c|c|c|c|c|c|c|c|c|c|c|c|c|c|c|c|}
\hline $\mathrm{TG}(44: 5)$ & 740.6 & 19.8 & $\mathrm{C} 47 \mathrm{H} 80 \mathrm{O} 6$ & $\begin{array}{l}\text { Glyceroli } \\
\text { pids }\end{array}$ & $\begin{array}{l}\text { Triacylgly } \\
\text { cerols }\end{array}$ & $\begin{array}{l}19951 \\
.9\end{array}$ & $\begin{array}{l}28676 . \\
2\end{array}$ & $\begin{array}{l}20587 . \\
8\end{array}$ & $\begin{array}{l}78511 . \\
7\end{array}$ & $\begin{array}{l}27391 . \\
9\end{array}$ & $\begin{array}{l}36532 . \\
2\end{array}$ & 0.0 & 0.0 & 0.0 & 0.0 & 0.0 & 0.0 \\
\hline TG(45:3) & 758.6 & 20.7 & C48H86O6 & $\begin{array}{l}\text { Glyceroli } \\
\text { pids }\end{array}$ & $\begin{array}{l}\text { Triacylgly } \\
\text { cerols }\end{array}$ & $\begin{array}{l}39704 \\
.8\end{array}$ & $\begin{array}{l}55370 . \\
9\end{array}$ & $\begin{array}{l}12429 . \\
4\end{array}$ & $\begin{array}{l}63761 . \\
3\end{array}$ & $\begin{array}{l}42972 . \\
2\end{array}$ & $\begin{array}{l}37277 . \\
1\end{array}$ & 0.0 & 0.0 & 0.0 & 0.0 & 0.0 & 0.0 \\
\hline $\mathrm{TG}(45: 4)$ & 756.6 & 20.5 & $\mathrm{C} 48 \mathrm{H} 84 \mathrm{O} 6$ & $\begin{array}{l}\text { Glyceroli } \\
\text { pids }\end{array}$ & $\begin{array}{l}\text { Triacylgly } \\
\text { cerols }\end{array}$ & $\begin{array}{l}42473 \\
.8\end{array}$ & $\begin{array}{l}53394 . \\
3\end{array}$ & $\begin{array}{l}33154 . \\
8\end{array}$ & $\begin{array}{l}94321 . \\
7\end{array}$ & $\begin{array}{l}53271 . \\
4\end{array}$ & $\begin{array}{l}78888 . \\
0\end{array}$ & 0.0 & 0.0 & 0.0 & 0.0 & 0.0 & 0.0 \\
\hline $\mathrm{TG}(46: 2)$ & 791.7 & 20.9 & С49H90O6 & $\begin{array}{l}\text { Glyceroli } \\
\text { pids }\end{array}$ & $\begin{array}{l}\text { Triacylgly } \\
\text { cerols }\end{array}$ & $\begin{array}{l}21004 \\
0.3\end{array}$ & $\begin{array}{l}238458 \\
.6\end{array}$ & $\begin{array}{l}129654 \\
.3\end{array}$ & $\begin{array}{l}384681 \\
.8\end{array}$ & $\begin{array}{l}205025 \\
.8\end{array}$ & $\begin{array}{l}331112 \\
.7\end{array}$ & 0.0 & 0.0 & 0.0 & 0.0 & 0.0 & 0.0 \\
\hline $\mathrm{TG}(46: 3)$ & 772.7 & 21.0 & С49H88O6 & $\begin{array}{l}\text { Glyceroli } \\
\text { pids }\end{array}$ & $\begin{array}{l}\text { Triacylgly } \\
\text { cerols }\end{array}$ & $\begin{array}{l}56254 \\
.4\end{array}$ & $\begin{array}{l}71446 . \\
4\end{array}$ & $\begin{array}{l}22545 . \\
9\end{array}$ & $\begin{array}{l}72535 . \\
6\end{array}$ & $\begin{array}{l}65212 . \\
8\end{array}$ & $\begin{array}{l}41613 . \\
3\end{array}$ & 0.0 & 0.0 & 0.0 & 0.0 & 0.0 & 0.0 \\
\hline TG(46:4) & 770.6 & 20.7 & C49H86O6 & $\begin{array}{l}\text { Glyceroli } \\
\text { pids }\end{array}$ & $\begin{array}{l}\text { Triacylgly } \\
\text { cerols }\end{array}$ & $\begin{array}{l}51655 \\
.8\end{array}$ & $\begin{array}{l}66298 . \\
0\end{array}$ & $\begin{array}{l}30197 . \\
6\end{array}$ & $\begin{array}{l}80631 . \\
0\end{array}$ & $\begin{array}{l}61228 . \\
3\end{array}$ & $\begin{array}{l}54504 . \\
8\end{array}$ & 0.0 & 0.0 & 0.0 & 0.0 & 0.0 & 0.0 \\
\hline TG(46:6) & 766.6 & 20.1 & $\mathrm{C} 49 \mathrm{H} 82 \mathrm{O} 6$ & $\begin{array}{l}\text { Glyceroli } \\
\text { pids }\end{array}$ & $\begin{array}{l}\text { Triacylgly } \\
\text { cerols }\end{array}$ & $\begin{array}{l}14905 \\
.2\end{array}$ & $\begin{array}{l}22126 . \\
6\end{array}$ & $\begin{array}{l}17299 . \\
6\end{array}$ & $\begin{array}{l}57318 . \\
4\end{array}$ & $\begin{array}{l}20277 . \\
5\end{array}$ & $\begin{array}{l}37260 . \\
5\end{array}$ & 0.0 & 0.0 & 0.0 & 0.0 & 0.0 & 0.0 \\
\hline $\mathrm{TG}(47: 1)$ & 807.7 & 21.3 & С50H94O6 & $\begin{array}{l}\text { Glyceroli } \\
\text { pids }\end{array}$ & $\begin{array}{l}\text { Triacylgly } \\
\text { cerols }\end{array}$ & $\begin{array}{l}52220 \\
4.6\end{array}$ & $\begin{array}{l}724560 \\
.1\end{array}$ & $\begin{array}{l}203496 \\
.5\end{array}$ & $\begin{array}{l}584987 \\
.8\end{array}$ & $\begin{array}{l}468681 \\
.9\end{array}$ & $\begin{array}{l}469245 \\
.1\end{array}$ & 0.0 & 0.0 & 0.0 & 0.0 & 0.0 & 0.0 \\
\hline $\mathrm{TG}(47: 3)$ & 786.7 & 21.1 & С50H90O6 & $\begin{array}{l}\text { Glyceroli } \\
\text { pids }\end{array}$ & $\begin{array}{l}\text { Triacylgly } \\
\text { cerols }\end{array}$ & $\begin{array}{l}89997 \\
.2\end{array}$ & $\begin{array}{l}114773 \\
.3\end{array}$ & $\begin{array}{l}33770 . \\
7\end{array}$ & $\begin{array}{l}98190 . \\
7\end{array}$ & $\begin{array}{l}75228 . \\
3\end{array}$ & $\begin{array}{l}64773 . \\
8\end{array}$ & 0.0 & 0.0 & 0.0 & 0.0 & 0.0 & 0.0 \\
\hline $\mathrm{TG}(47: 4)$ & 784.7 & 20.9 & С50H88O6 & $\begin{array}{l}\text { Glyceroli } \\
\text { pids }\end{array}$ & $\begin{array}{l}\text { Triacylgly } \\
\text { cerols }\end{array}$ & $\begin{array}{l}55127 \\
.9\end{array}$ & $\begin{array}{l}79185 . \\
9\end{array}$ & $\begin{array}{l}29497 . \\
2\end{array}$ & $\begin{array}{l}106812 \\
.3\end{array}$ & $\begin{array}{l}61778 . \\
3\end{array}$ & $\begin{array}{l}78131 . \\
8\end{array}$ & 0.0 & 0.0 & 0.0 & 0.0 & 0.0 & 0.0 \\
\hline $\mathrm{TG}(47: 5)$ & 782.6 & 20.7 & C50H86O6 & $\begin{array}{l}\text { Glyceroli } \\
\text { pids }\end{array}$ & $\begin{array}{l}\text { Triacylgly } \\
\text { cerols }\end{array}$ & $\begin{array}{l}37188 \\
.8\end{array}$ & $\begin{array}{l}52057 . \\
2\end{array}$ & $\begin{array}{l}35156 . \\
2\end{array}$ & $\begin{array}{l}79414 . \\
3\end{array}$ & $\begin{array}{l}45416 . \\
5\end{array}$ & $\begin{array}{l}79732 . \\
4\end{array}$ & 0.0 & 0.0 & 0.0 & 0.0 & 0.0 & 0.0 \\
\hline $\mathrm{TG}(48: 1)$ & 821.7 & 21.4 & C51H96O6 & $\begin{array}{l}\text { Glyceroli } \\
\text { pids }\end{array}$ & $\begin{array}{l}\text { Triacylgly } \\
\text { cerols }\end{array}$ & $\begin{array}{l}86424 \\
3.6\end{array}$ & $\begin{array}{l}107005 \\
2.8\end{array}$ & $\begin{array}{l}273265 \\
.1\end{array}$ & $\begin{array}{l}809683 \\
.3\end{array}$ & $\begin{array}{l}674781 \\
.4\end{array}$ & $\begin{array}{l}526191 \\
.2\end{array}$ & 0.0 & 0.0 & 0.0 & 0.0 & 0.0 & 0.0 \\
\hline $\mathrm{TG}(48: 3)$ & 800.7 & 21.3 & С51H92O6 & $\begin{array}{l}\text { Glyceroli } \\
\text { pids }\end{array}$ & $\begin{array}{l}\text { Triacylgly } \\
\text { cerols }\end{array}$ & $\begin{array}{l}70755 \\
.1\end{array}$ & $\begin{array}{l}99728 . \\
1\end{array}$ & $\begin{array}{l}34185 . \\
3\end{array}$ & $\begin{array}{l}76859 . \\
5\end{array}$ & $\begin{array}{l}75725 . \\
6\end{array}$ & $\begin{array}{l}53667 . \\
7\end{array}$ & 0.0 & 0.0 & 0.0 & 0.0 & 0.0 & 0.0 \\
\hline TG(48:4) & 798.7 & 21.1 & С51H90O6 & $\begin{array}{l}\text { Glyceroli } \\
\text { pids }\end{array}$ & $\begin{array}{l}\text { Triacylgly } \\
\text { cerols }\end{array}$ & $\begin{array}{l}92322 \\
.9\end{array}$ & $\begin{array}{l}99083 . \\
7\end{array}$ & $\begin{array}{l}35921 . \\
1\end{array}$ & $\begin{array}{l}109878 \\
.8\end{array}$ & $\begin{array}{l}99322 . \\
2\end{array}$ & $\begin{array}{l}72924 . \\
5\end{array}$ & 0.0 & 0.0 & 0.0 & 0.0 & 0.0 & 0.0 \\
\hline $\mathrm{TG}(48: 5)$ & 796.7 & 20.9 & С51H88O6 & $\begin{array}{l}\text { Glyceroli } \\
\text { pids }\end{array}$ & $\begin{array}{l}\text { Triacylgly } \\
\text { cerols }\end{array}$ & $\begin{array}{l}49447 \\
.3\end{array}$ & $\begin{array}{l}68857 . \\
7\end{array}$ & $\begin{array}{l}40110 . \\
6\end{array}$ & $\begin{array}{l}87246 . \\
1\end{array}$ & $\begin{array}{l}59935 . \\
7\end{array}$ & $\begin{array}{l}77212 . \\
5\end{array}$ & 0.0 & 0.0 & 0.0 & 0.0 & 0.0 & 0.0 \\
\hline TG(48:6) & 794.6 & 20.6 & C51H86O6 & $\begin{array}{l}\text { Glyceroli } \\
\text { pids }\end{array}$ & $\begin{array}{l}\text { Triacylgly } \\
\text { cerols }\end{array}$ & $\begin{array}{l}29430 \\
.3\end{array}$ & $\begin{array}{l}29797 . \\
0\end{array}$ & $\begin{array}{l}23883 . \\
6\end{array}$ & $\begin{array}{l}50489 . \\
0\end{array}$ & $\begin{array}{l}37510 . \\
1\end{array}$ & $\begin{array}{l}39762 . \\
9\end{array}$ & 0.0 & 0.0 & 0.0 & 0.0 & 0.0 & 0.0 \\
\hline TG(49:3) & 814.7 & 21.4 & С52H94O6 & $\begin{array}{l}\text { Glyceroli } \\
\text { pids }\end{array}$ & $\begin{array}{l}\text { Triacylgly } \\
\text { cerols }\end{array}$ & $\begin{array}{l}86881 \\
.9\end{array}$ & $\begin{array}{l}95773 . \\
2\end{array}$ & $\begin{array}{l}26416 . \\
2\end{array}$ & $\begin{array}{l}65959 . \\
8\end{array}$ & $\begin{array}{l}79574 . \\
5\end{array}$ & $\begin{array}{l}51361 . \\
4\end{array}$ & 0.0 & 0.0 & 0.0 & 0.0 & 0.0 & 0.0 \\
\hline TG(49:4) & 812.7 & 21.3 & С52H92O6 & $\begin{array}{l}\text { Glyceroli } \\
\text { pids }\end{array}$ & $\begin{array}{l}\text { Triacylgly } \\
\text { cerols }\end{array}$ & $\begin{array}{l}12379 \\
5.4\end{array}$ & $\begin{array}{l}150628 \\
.8\end{array}$ & $\begin{array}{l}50941 . \\
1\end{array}$ & $\begin{array}{l}138167 \\
.5\end{array}$ & $\begin{array}{l}110086 \\
.1\end{array}$ & $\begin{array}{l}106135 \\
.0\end{array}$ & 0.0 & 0.0 & 0.0 & 0.0 & 0.0 & 0.0 \\
\hline
\end{tabular}




\begin{tabular}{|c|c|c|c|c|c|c|c|c|c|c|c|c|c|c|c|c|c|}
\hline $\mathrm{TG}(49: 5)$ & 810.7 & 21.1 & C52H90O6 & $\begin{array}{l}\text { Glyceroli } \\
\text { pids }\end{array}$ & $\begin{array}{l}\text { Triacylgly } \\
\text { cerols }\end{array}$ & $\begin{array}{l}48408 \\
.7\end{array}$ & $\begin{array}{l}67999 . \\
3\end{array}$ & $\begin{array}{l}36037 . \\
1\end{array}$ & $\begin{array}{l}83199 . \\
1\end{array}$ & $\begin{array}{l}57396 . \\
5\end{array}$ & $\begin{array}{l}76185 . \\
8\end{array}$ & 0.0 & 0.0 & 0.0 & 0.0 & 0.0 & 0.0 \\
\hline $\mathrm{TG}(50: 2)$ & 847.8 & 21.5 & $\mathrm{C} 53 \mathrm{H} 98 \mathrm{O} 6$ & $\begin{array}{l}\text { Glyceroli } \\
\text { pids }\end{array}$ & $\begin{array}{l}\text { Triacylgly } \\
\text { cerols }\end{array}$ & $\begin{array}{l}76021 \\
4.3\end{array}$ & $\begin{array}{l}950530 \\
.9\end{array}$ & $\begin{array}{l}274543 \\
.2\end{array}$ & $\begin{array}{l}682455 \\
.3\end{array}$ & $\begin{array}{l}594308 \\
.0\end{array}$ & $\begin{array}{l}478292 \\
.1\end{array}$ & 0.0 & 0.0 & 0.0 & 0.0 & 0.0 & 0.0 \\
\hline $\mathrm{TG}(50: 3)$ & 828.7 & 21.6 & C53H96O6 & $\begin{array}{l}\text { Glyceroli } \\
\text { pids }\end{array}$ & $\begin{array}{l}\text { Triacylgly } \\
\text { cerols }\end{array}$ & $\begin{array}{l}27404 \\
6.8\end{array}$ & $\begin{array}{l}364674 \\
.2\end{array}$ & $\begin{array}{l}105213 \\
.3\end{array}$ & $\begin{array}{l}328663 \\
.4\end{array}$ & $\begin{array}{l}279472 \\
.8\end{array}$ & $\begin{array}{l}180582 \\
.0\end{array}$ & 0.0 & 0.0 & 0.0 & 0.0 & 0.0 & 0.0 \\
\hline $\mathrm{TG}(50: 4)$ & 843.7 & 21.2 & C53H94O6 & $\begin{array}{l}\text { Glyceroli } \\
\text { pids }\end{array}$ & $\begin{array}{l}\text { Triacylgly } \\
\text { cerols }\end{array}$ & $\begin{array}{l}13493 \\
0.1\end{array}$ & $\begin{array}{l}176819 \\
.4\end{array}$ & $\begin{array}{l}57875 . \\
2\end{array}$ & $\begin{array}{l}144476 \\
.6\end{array}$ & $\begin{array}{l}126790 \\
.0\end{array}$ & $\begin{array}{l}110931 \\
.2\end{array}$ & 0.0 & 0.0 & 0.0 & 0.0 & 0.0 & 0.0 \\
\hline $\mathrm{TG}(50: 5)$ & 824.7 & 21.2 & $\mathrm{C} 53 \mathrm{H} 92 \mathrm{O} 6$ & $\begin{array}{l}\text { Glyceroli } \\
\text { pids }\end{array}$ & $\begin{array}{l}\text { Triacylgly } \\
\text { cerols }\end{array}$ & $\begin{array}{l}86669 \\
.4\end{array}$ & $\begin{array}{l}102594 \\
.0\end{array}$ & $\begin{array}{l}39570 . \\
9\end{array}$ & $\begin{array}{l}117137 \\
.6\end{array}$ & $\begin{array}{l}85160 . \\
6\end{array}$ & $\begin{array}{l}78812 . \\
7\end{array}$ & 0.0 & 0.0 & 0.0 & 0.0 & 0.0 & 0.0 \\
\hline $\mathrm{TG}(51: 5)$ & 838.7 & 21.4 & $\mathrm{C} 54 \mathrm{H} 94 \mathrm{O} 6$ & $\begin{array}{l}\text { Glyceroli } \\
\text { pids }\end{array}$ & $\begin{array}{l}\text { Triacylgly } \\
\text { cerols }\end{array}$ & $\begin{array}{l}50201 \\
.6\end{array}$ & $\begin{array}{l}86808 . \\
9\end{array}$ & $\begin{array}{l}27732 . \\
9\end{array}$ & $\begin{array}{l}111530 \\
.1\end{array}$ & $\begin{array}{l}70839 . \\
0\end{array}$ & $\begin{array}{l}41736 . \\
8\end{array}$ & 0.0 & 0.0 & 0.0 & 0.0 & 0.0 & 0.0 \\
\hline $\mathrm{TG}(51: 6)$ & 836.7 & 21.2 & C54H92O6 & $\begin{array}{l}\text { Glyceroli } \\
\text { pids }\end{array}$ & $\begin{array}{l}\text { Triacylgly } \\
\text { cerols }\end{array}$ & $\begin{array}{l}34209 \\
.2\end{array}$ & $\begin{array}{l}41972 . \\
6\end{array}$ & $\begin{array}{l}17335 . \\
9\end{array}$ & $\begin{array}{l}48941 . \\
4\end{array}$ & $\begin{array}{l}43413 . \\
5\end{array}$ & $\begin{array}{l}31010 . \\
3\end{array}$ & 0.0 & 0.0 & 0.0 & 0.0 & 0.0 & 0.0 \\
\hline $\mathrm{TG}(51: 7)$ & 834.7 & 21.0 & C54H90O6 & $\begin{array}{l}\text { Glyceroli } \\
\text { pids }\end{array}$ & $\begin{array}{l}\text { Triacylgly } \\
\text { cerols }\end{array}$ & $\begin{array}{l}14294 \\
.4\end{array}$ & $\begin{array}{l}23285 . \\
5\end{array}$ & 0.0 & $\begin{array}{l}28395 . \\
9\end{array}$ & $\begin{array}{l}18778 . \\
1\end{array}$ & $\begin{array}{l}11187 . \\
0\end{array}$ & 0.0 & 0.0 & 0.0 & 0.0 & 0.0 & 0.0 \\
\hline $\mathrm{TG}(52: 2)$ & 875.8 & 21.8 & $\mathrm{C} 55 \mathrm{H} 102 \mathrm{O} 6$ & $\begin{array}{l}\text { Glyceroli } \\
\text { pids }\end{array}$ & $\begin{array}{l}\text { Triacylgly } \\
\text { cerols }\end{array}$ & $\begin{array}{l}82969 \\
0.5\end{array}$ & $\begin{array}{l}988813 \\
.9\end{array}$ & $\begin{array}{l}387844 \\
.1\end{array}$ & $\begin{array}{l}612009 \\
.3\end{array}$ & $\begin{array}{l}628960 \\
.0\end{array}$ & $\begin{array}{l}471697 \\
.7\end{array}$ & 0.0 & 0.0 & 0.0 & 0.0 & 0.0 & 0.0 \\
\hline $\mathrm{TG}(52: 4)$ & 854.7 & 21.7 & C55H98O6 & $\begin{array}{l}\text { Glyceroli } \\
\text { pids }\end{array}$ & $\begin{array}{l}\text { Triacylgly } \\
\text { cerols }\end{array}$ & $\begin{array}{l}10781 \\
9.8\end{array}$ & $\begin{array}{l}138935 \\
.6\end{array}$ & $\begin{array}{l}58238 . \\
4\end{array}$ & $\begin{array}{l}102777 \\
.5\end{array}$ & $\begin{array}{l}107870 \\
.5\end{array}$ & $\begin{array}{l}80451 . \\
8\end{array}$ & 0.0 & 0.0 & 0.0 & 0.0 & 0.0 & 0.0 \\
\hline $\mathrm{TG}(52: 5)$ & 852.7 & 21.5 & $\mathrm{C} 55 \mathrm{H} 96 \mathrm{O} 6$ & $\begin{array}{l}\text { Glyceroli } \\
\text { pids }\end{array}$ & $\begin{array}{l}\text { Triacylgly } \\
\text { cerols }\end{array}$ & $\begin{array}{l}12181 \\
0.6\end{array}$ & $\begin{array}{l}154757 \\
.2\end{array}$ & $\begin{array}{l}64254 . \\
8\end{array}$ & $\begin{array}{l}135813 \\
.6\end{array}$ & $\begin{array}{l}125196 \\
.7\end{array}$ & $\begin{array}{l}94939 . \\
6\end{array}$ & 0.0 & 0.0 & 0.0 & 0.0 & 0.0 & 0.0 \\
\hline TG(52:6) & 850.7 & 21.3 & C55H94O6 & $\begin{array}{l}\text { Glyceroli } \\
\text { pids }\end{array}$ & $\begin{array}{l}\text { Triacylgly } \\
\text { cerols }\end{array}$ & $\begin{array}{l}59532 \\
.2\end{array}$ & $\begin{array}{l}72246 . \\
9\end{array}$ & $\begin{array}{l}29010 . \\
4\end{array}$ & $\begin{array}{l}74380 . \\
2\end{array}$ & $\begin{array}{l}67573 . \\
9\end{array}$ & $\begin{array}{l}44922 . \\
4\end{array}$ & 0.0 & 0.0 & 0.0 & 0.0 & 0.0 & 0.0 \\
\hline $\mathrm{TG}(52: 7)$ & 848.7 & 21.1 & C55H92O6 & $\begin{array}{l}\text { Glyceroli } \\
\text { pids }\end{array}$ & $\begin{array}{l}\text { Triacylgly } \\
\text { cerols }\end{array}$ & $\begin{array}{l}42698 \\
.3\end{array}$ & $\begin{array}{l}43787 . \\
7\end{array}$ & $\begin{array}{l}19513 . \\
5\end{array}$ & $\begin{array}{l}53732 . \\
7\end{array}$ & $\begin{array}{l}46337 . \\
3\end{array}$ & $\begin{array}{l}24653 . \\
2\end{array}$ & 0.0 & 0.0 & 0.0 & 0.0 & 0.0 & 0.0 \\
\hline $\mathrm{TG}(53: 2)$ & 889.8 & 21.9 & C56H104O6 & $\begin{array}{l}\text { Glyceroli } \\
\text { pids }\end{array}$ & $\begin{array}{l}\text { Triacylgly } \\
\text { cerols }\end{array}$ & $\begin{array}{l}36051 \\
0.5\end{array}$ & $\begin{array}{l}573796 \\
.3\end{array}$ & $\begin{array}{l}168541 \\
.1\end{array}$ & $\begin{array}{l}312725 \\
.6\end{array}$ & $\begin{array}{l}336958 \\
.2\end{array}$ & $\begin{array}{l}160627 \\
.3\end{array}$ & 0.0 & 0.0 & 0.0 & 0.0 & 0.0 & 0.0 \\
\hline $\mathrm{TG}(53: 4)$ & 868.7 & 21.8 & C56H100O6 & $\begin{array}{l}\text { Glyceroli } \\
\text { pids }\end{array}$ & $\begin{array}{l}\text { Triacylgly } \\
\text { cerols }\end{array}$ & $\begin{array}{l}66740 \\
.6\end{array}$ & $\begin{array}{l}86449 . \\
3\end{array}$ & $\begin{array}{l}30665 . \\
3\end{array}$ & $\begin{array}{l}57411 . \\
4\end{array}$ & $\begin{array}{l}63478 . \\
2\end{array}$ & $\begin{array}{l}41362 . \\
8\end{array}$ & 0.0 & 0.0 & 0.0 & 0.0 & 0.0 & 0.0 \\
\hline $\mathrm{TG}(53: 5)$ & 866.7 & 21.6 & C56H98O6 & $\begin{array}{l}\text { Glyceroli } \\
\text { pids }\end{array}$ & $\begin{array}{l}\text { Triacylgly } \\
\text { cerols }\end{array}$ & $\begin{array}{l}11585 \\
3.9\end{array}$ & $\begin{array}{l}153350 \\
.4\end{array}$ & $\begin{array}{l}42180 . \\
3\end{array}$ & $\begin{array}{l}105551 \\
.1\end{array}$ & $\begin{array}{l}113615 \\
.7\end{array}$ & $\begin{array}{l}56965 . \\
9\end{array}$ & 0.0 & 0.0 & 0.0 & 0.0 & 0.0 & 0.0 \\
\hline TG(53:6) & 864.7 & 21.4 & $\mathrm{C} 56 \mathrm{H} 96 \mathrm{O} 6$ & $\begin{array}{l}\text { Glyceroli } \\
\text { pids }\end{array}$ & $\begin{array}{l}\text { Triacylgly } \\
\text { cerols }\end{array}$ & $\begin{array}{l}56349 \\
.1\end{array}$ & $\begin{array}{l}80864 . \\
8\end{array}$ & $\begin{array}{l}25054 . \\
2\end{array}$ & $\begin{array}{l}71201 . \\
1\end{array}$ & $\begin{array}{l}70209 . \\
3\end{array}$ & $\begin{array}{l}31277 . \\
4\end{array}$ & 0.0 & 0.0 & 0.0 & 0.0 & 0.0 & 0.0 \\
\hline $\mathrm{TG}(53: 7)$ & 862.7 & 21.3 & $\mathrm{C} 56 \mathrm{H} 94 \mathrm{O} 6$ & $\begin{array}{l}\text { Glyceroli } \\
\text { pids }\end{array}$ & $\begin{array}{l}\text { Triacylgly } \\
\text { cerols }\end{array}$ & $\begin{array}{l}32785 \\
.9\end{array}$ & $\begin{array}{l}46303 . \\
6\end{array}$ & $\begin{array}{l}12931 . \\
9\end{array}$ & $\begin{array}{l}39260 . \\
9\end{array}$ & $\begin{array}{l}45411 . \\
8\end{array}$ & $\begin{array}{l}21658 . \\
1\end{array}$ & 0.0 & 0.0 & 0.0 & 0.0 & 0.0 & 0.0 \\
\hline
\end{tabular}




\begin{tabular}{|c|c|c|c|c|c|c|c|c|c|c|c|c|c|c|c|c|c|}
\hline $\mathrm{TG}(53: 8)$ & 860.7 & 21.1 & C56H92O6 & $\begin{array}{l}\text { Glyceroli } \\
\text { pids }\end{array}$ & $\begin{array}{l}\text { Triacylgly } \\
\text { cerols }\end{array}$ & $\begin{array}{l}17089 \\
.3\end{array}$ & $\begin{array}{l}19413 . \\
8\end{array}$ & 0.0 & $\begin{array}{l}30893 . \\
1\end{array}$ & $\begin{array}{l}19546 . \\
9\end{array}$ & 0.0 & 0.0 & 0.0 & 0.0 & 0.0 & 0.0 & 0.0 \\
\hline $\mathrm{TG}(54: 2)$ & 903.8 & 22.0 & C57H106O6 & $\begin{array}{l}\text { Glyceroli } \\
\text { pids }\end{array}$ & $\begin{array}{l}\text { Triacylgly } \\
\text { cerols }\end{array}$ & $\begin{array}{l}29942 \\
2.2\end{array}$ & $\begin{array}{l}423178 \\
.0\end{array}$ & $\begin{array}{l}166784 \\
.9\end{array}$ & $\begin{array}{l}231048 \\
.4\end{array}$ & $\begin{array}{l}262889 \\
.1\end{array}$ & $\begin{array}{l}177627 \\
.4\end{array}$ & 0.0 & 0.0 & 0.0 & 0.0 & 0.0 & 0.0 \\
\hline $\mathrm{TG}(54: 5)$ & 880.8 & 21.7 & C57H100O6 & $\begin{array}{l}\text { Glyceroli } \\
\text { pids }\end{array}$ & $\begin{array}{l}\text { Triacylgly } \\
\text { cerols }\end{array}$ & $\begin{array}{l}13319 \\
6.2\end{array}$ & $\begin{array}{l}169650 \\
.0\end{array}$ & $\begin{array}{l}96363 . \\
5\end{array}$ & $\begin{array}{l}121434 \\
.2\end{array}$ & $\begin{array}{l}129676 \\
.0\end{array}$ & $\begin{array}{l}110231 \\
.2\end{array}$ & 0.0 & 0.0 & 0.0 & 0.0 & 0.0 & 0.0 \\
\hline $\mathrm{TG}(54: 7)$ & 876.7 & 21.4 & C57H96O6 & $\begin{array}{l}\text { Glyceroli } \\
\text { pids }\end{array}$ & $\begin{array}{l}\text { Triacylgly } \\
\text { cerols }\end{array}$ & $\begin{array}{l}64479 \\
.8\end{array}$ & $\begin{array}{l}68668 . \\
0\end{array}$ & $\begin{array}{l}28509 . \\
8\end{array}$ & $\begin{array}{l}62562 . \\
6\end{array}$ & $\begin{array}{l}67171 . \\
7\end{array}$ & $\begin{array}{l}35841 . \\
1\end{array}$ & 0.0 & 0.0 & 0.0 & 0.0 & 0.0 & 0.0 \\
\hline TG(54:8) & 874.7 & 21.3 & C57H94O6 & $\begin{array}{l}\text { Glyceroli } \\
\text { pids }\end{array}$ & $\begin{array}{l}\text { Triacylgly } \\
\text { cerols }\end{array}$ & $\begin{array}{l}32771 \\
.9\end{array}$ & $\begin{array}{l}37495 . \\
1\end{array}$ & $\begin{array}{l}11556 . \\
7\end{array}$ & $\begin{array}{l}37760 . \\
2\end{array}$ & $\begin{array}{l}35770 . \\
2\end{array}$ & $\begin{array}{l}21938 . \\
7\end{array}$ & 0.0 & 0.0 & 0.0 & 0.0 & 0.0 & 0.0 \\
\hline $\mathrm{TG}(55: 2)$ & 917.8 & 22.1 & C58H108O6 & $\begin{array}{l}\text { Glyceroli } \\
\text { pids }\end{array}$ & $\begin{array}{l}\text { Triacylgly } \\
\text { cerols }\end{array}$ & $\begin{array}{l}83732 \\
.7\end{array}$ & $\begin{array}{l}131967 \\
.9\end{array}$ & $\begin{array}{l}37728 . \\
5\end{array}$ & $\begin{array}{l}69762 . \\
6\end{array}$ & $\begin{array}{l}84474 . \\
7\end{array}$ & $\begin{array}{l}41231 . \\
4\end{array}$ & 0.0 & 0.0 & 0.0 & 0.0 & 0.0 & 0.0 \\
\hline TG(55:5) & 894.8 & 21.9 & $\mathrm{C} 58 \mathrm{H} 102 \mathrm{O} 6$ & $\begin{array}{l}\text { Glyceroli } \\
\text { pids }\end{array}$ & $\begin{array}{l}\text { Triacylgly } \\
\text { cerols }\end{array}$ & $\begin{array}{l}59340 \\
.5\end{array}$ & $\begin{array}{l}84104 . \\
2\end{array}$ & $\begin{array}{l}36746 . \\
9\end{array}$ & $\begin{array}{l}64373 . \\
1\end{array}$ & $\begin{array}{l}71974 . \\
4\end{array}$ & $\begin{array}{l}33116 . \\
4\end{array}$ & 0.0 & 0.0 & 0.0 & 0.0 & 0.0 & 0.0 \\
\hline $\mathrm{TG}(55: 6)$ & 892.8 & 21.7 & C58H100O6 & $\begin{array}{l}\text { Glyceroli } \\
\text { pids }\end{array}$ & $\begin{array}{l}\text { Triacylgly } \\
\text { cerols }\end{array}$ & $\begin{array}{l}64663 \\
.6\end{array}$ & $\begin{array}{l}100788 \\
.8\end{array}$ & $\begin{array}{l}42025 . \\
7\end{array}$ & $\begin{array}{l}64788 . \\
3\end{array}$ & $\begin{array}{l}65175 . \\
5\end{array}$ & $\begin{array}{l}33992 . \\
4\end{array}$ & 0.0 & 0.0 & 0.0 & 0.0 & 0.0 & 0.0 \\
\hline $\mathrm{TG}(55: 7)$ & 890.7 & 21.6 & $\mathrm{C} 58 \mathrm{H} 98 \mathrm{O} 6$ & $\begin{array}{l}\text { Glyceroli } \\
\text { pids }\end{array}$ & $\begin{array}{l}\text { Triacylgly } \\
\text { cerols }\end{array}$ & $\begin{array}{l}10430 \\
1.8\end{array}$ & $\begin{array}{l}156543 \\
.3\end{array}$ & $\begin{array}{l}41846 . \\
5\end{array}$ & $\begin{array}{l}112593 \\
.2\end{array}$ & $\begin{array}{l}86556 . \\
4\end{array}$ & $\begin{array}{l}57836 . \\
5\end{array}$ & 0.0 & 0.0 & 0.0 & 0.0 & 0.0 & 0.0 \\
\hline TG(55:8) & 888.7 & 21.4 & C58H96O6 & $\begin{array}{l}\text { Glyceroli } \\
\text { pids }\end{array}$ & $\begin{array}{l}\text { Triacylgly } \\
\text { cerols }\end{array}$ & $\begin{array}{l}53635 \\
.1\end{array}$ & $\begin{array}{l}56177 . \\
3\end{array}$ & $\begin{array}{l}18855 . \\
9\end{array}$ & $\begin{array}{l}47245 . \\
5\end{array}$ & $\begin{array}{l}55964 . \\
3\end{array}$ & $\begin{array}{l}22808 . \\
5\end{array}$ & 0.0 & 0.0 & 0.0 & 0.0 & 0.0 & 0.0 \\
\hline TG(56:6) & 906.8 & 21.8 & С59H102O6 & $\begin{array}{l}\text { Glyceroli } \\
\text { pids }\end{array}$ & $\begin{array}{l}\text { Triacylgly } \\
\text { cerols }\end{array}$ & $\begin{array}{l}97648 \\
.4\end{array}$ & $\begin{array}{l}102519 \\
.7\end{array}$ & $\begin{array}{l}69285 . \\
9\end{array}$ & $\begin{array}{l}88190 . \\
8\end{array}$ & $\begin{array}{l}95536 . \\
8\end{array}$ & $\begin{array}{l}72159 . \\
4\end{array}$ & 0.0 & 0.0 & 0.0 & 0.0 & 0.0 & 0.0 \\
\hline $\mathrm{TG}(56: 7)$ & 904.8 & 21.7 & C59H100O6 & $\begin{array}{l}\text { Glyceroli } \\
\text { pids }\end{array}$ & $\begin{array}{l}\text { Triacylgly } \\
\text { cerols }\end{array}$ & $\begin{array}{l}71701 \\
.5\end{array}$ & $\begin{array}{l}77249 . \\
2\end{array}$ & $\begin{array}{l}42838 . \\
2\end{array}$ & $\begin{array}{l}66327 . \\
6\end{array}$ & $\begin{array}{l}77287 . \\
4\end{array}$ & $\begin{array}{l}67446 . \\
9\end{array}$ & 0.0 & 0.0 & 0.0 & 0.0 & 0.0 & 0.0 \\
\hline TG(56:8) & 902.7 & 21.5 & C59H98O6 & $\begin{array}{l}\text { Glyceroli } \\
\text { pids }\end{array}$ & $\begin{array}{l}\text { Triacylgly } \\
\text { cerols }\end{array}$ & $\begin{array}{l}50749 \\
.8\end{array}$ & $\begin{array}{l}69632 . \\
7\end{array}$ & $\begin{array}{l}26266 . \\
2\end{array}$ & $\begin{array}{l}56400 . \\
7\end{array}$ & $\begin{array}{l}64195 . \\
7\end{array}$ & $\begin{array}{l}33395 . \\
0\end{array}$ & 0.0 & 0.0 & 0.0 & 0.0 & 0.0 & 0.0 \\
\hline $\mathrm{TG}(57: 6)$ & 920.8 & 21.9 & C60H104O6 & $\begin{array}{l}\text { Glyceroli } \\
\text { pids }\end{array}$ & $\begin{array}{l}\text { Triacylgly } \\
\text { cerols }\end{array}$ & $\begin{array}{l}30164 \\
.4\end{array}$ & $\begin{array}{l}35159 . \\
4\end{array}$ & $\begin{array}{l}18408 . \\
9\end{array}$ & $\begin{array}{l}27462 . \\
3\end{array}$ & $\begin{array}{l}29862 . \\
1\end{array}$ & 0.0 & 0.0 & 0.0 & 0.0 & 0.0 & 0.0 & 0.0 \\
\hline $\mathrm{TG}(58: 10)$ & 926.7 & 21.5 & C61H98O6 & $\begin{array}{l}\text { Glyceroli } \\
\text { pids }\end{array}$ & $\begin{array}{l}\text { Triacylgly } \\
\text { cerols }\end{array}$ & $\begin{array}{l}36839 \\
.9\end{array}$ & $\begin{array}{l}37485 . \\
5\end{array}$ & $\begin{array}{l}14786 . \\
6\end{array}$ & $\begin{array}{l}48375 . \\
3\end{array}$ & $\begin{array}{l}42247 . \\
9\end{array}$ & $\begin{array}{l}15033 . \\
9\end{array}$ & 0.0 & 0.0 & 0.0 & 0.0 & 0.0 & 0.0 \\
\hline Tricosene & 339.4 & 7.3 & $\mathrm{C} 23 \mathrm{H} 46$ & $\begin{array}{l}\text { Fatty } \\
\text { Acyls }\end{array}$ & $\begin{array}{l}\text { Hydrocarb } \\
\text { ons }\end{array}$ & $\begin{array}{l}34763 \\
.7\end{array}$ & $\begin{array}{l}14292 . \\
1\end{array}$ & $\begin{array}{l}41328 . \\
8\end{array}$ & $\begin{array}{l}52580 . \\
1\end{array}$ & $\begin{array}{l}29064 . \\
1\end{array}$ & $\begin{array}{l}125971 \\
.1\end{array}$ & 0.0 & 0.0 & 0.0 & 0.0 & 0.0 & 0.0 \\
\hline Ulexone B & 424.1 & 1.8 & $\mathrm{C} 25 \mathrm{H} 22 \mathrm{O} 5$ & $\begin{array}{l}\text { Polyketid } \\
\text { es }\end{array}$ & Flavonoids & 0.0 & 0.0 & 0.0 & 0.0 & 0.0 & 0.0 & $\begin{array}{l}109376 \\
6.4\end{array}$ & $\begin{array}{l}721329 \\
.3\end{array}$ & $\begin{array}{l}102031 \\
4.8\end{array}$ & $\begin{array}{l}111995 \\
0.9\end{array}$ & $\begin{array}{l}971582 \\
.1\end{array}$ & $\begin{array}{l}131039 \\
4.1\end{array}$ \\
\hline
\end{tabular}


Table S3. Unidentified features detected in lipidomics analysis (reverse phase HPLC MS run) in ESP samples.

\begin{tabular}{|c|c|c|c|c|c|}
\hline $\mathbf{m} / \mathbf{z}$ & Polarity & RT & Average intensity & $\begin{array}{l}\text { Plausible Formula } \\
\text { (neutral) }\end{array}$ & Delta ppm \\
\hline 1055.299683 & positive & 20.59 & $1.1 \mathrm{E}+06$ & & \\
\hline 453.1331391 & negative & 1.70 & $6.4 \mathrm{E}+05$ & $\mathrm{C}_{20} \mathrm{H}_{26} \mathrm{~N}_{2} \mathrm{O}_{8} \mathrm{~S}$ & -1.27 \\
\hline 674.1807676 & positive & 9.90 & $6.0 \mathrm{E}+05$ & & \\
\hline 654.3332565 & positive & 7.94 & $5.4 \mathrm{E}+05$ & $\mathrm{C}_{30} \mathrm{H}_{47} \mathrm{O}_{11} \mathrm{~N}_{5}$ & -1.88 \\
\hline 526.1430607 & positive & 7.34 & $4.0 \mathrm{E}+05$ & $\mathrm{C}_{21} \mathrm{H}_{35} \mathrm{O}_{6} \mathrm{NS}_{4}$ & 2.01 \\
\hline 748.1998119 & positive & 11.07 & $3.8 \mathrm{E}+05$ & & \\
\hline 487.0697073 & negative & 2.83 & $3.7 \mathrm{E}+05$ & & \\
\hline 460.0367553 & negative & 3.14 & $2.4 \mathrm{E}+05$ & & \\
\hline 325.3109054 & positive & 8.64 & $1.9 \mathrm{E}+05$ & $\mathrm{C}_{21} \mathrm{H}_{40} \mathrm{O}_{2}$ & 2.46 \\
\hline 1251.301854 & positive & 21.45 & $1.9 \mathrm{E}+05$ & & \\
\hline 452.1239479 & positive & 6.13 & $1.7 \mathrm{E}+05$ & & \\
\hline 646.1467 & positive & 10.89 & $1.5 \mathrm{E}+05$ & $\mathrm{C}_{27} \mathrm{H}_{36} \mathrm{NO}_{11} \mathrm{~S}_{3}$ & 3.40 \\
\hline 565.0809657 & negative & 2.98 & $1.4 \mathrm{E}+05$ & & \\
\hline 681.2982221 & negative & 7.95 & $1.3 \mathrm{E}+05$ & & \\
\hline 822.2186844 & positive & 12.38 & $1.3 \mathrm{E}+05$ & & \\
\hline 540.1585902 & positive & 9.15 & $1.0 \mathrm{E}+05$ & $\mathrm{C}_{22} \mathrm{H}_{29} \mathrm{~N}_{5} \mathrm{O}_{7} \mathrm{~S}_{2}$ & 0.87 \\
\hline 365.3042117 & positive & 8.55 & $1.0 \mathrm{E}+05$ & $\mathrm{C}_{23} \mathrm{H}_{40} \mathrm{O}_{3}$ & -2.22 \\
\hline 614.1779601 & positive & 10.49 & $1.0 \mathrm{E}+05$ & & \\
\hline 662.1773142 & positive & 9.02 & $9.5 \mathrm{E}+04$ & & \\
\hline 475.0666702 & negative & 3.24 & $9.4 \mathrm{E}+04$ & & \\
\hline 908.2405264 & positive & 15.15 & $9.1 \mathrm{E}+04$ & & \\
\hline
\end{tabular}

Note: Plausible formulas matched with isotope pattern are given where possible. Delta ppm is the difference between detected mass and theoretical mass for that formula. 